Copyright

by

Ali Moinfar

2013 
The Dissertation Committee for Ali Moinfar Certifies that this is the approved version of the following dissertation:

Development of an Efficient Embedded Discrete Fracture Model for 3D Compositional Reservoir Simulation in Fractured Reservoirs

Committee:

Kamy Sepehrnoori, Supervisor

Russell T. Johns, Co-Supervisor

Mojdeh Delshad

Chun Huh

Todd J. Arbogast 
Development of an Efficient Embedded Discrete Fracture Model for 3D

Compositional Reservoir Simulation in Fractured Reservoirs

by

Ali Moinfar, B.E.E; M.S.E.

\author{
Dissertation \\ Presented to the Faculty of the Graduate School of \\ The University of Texas at Austin \\ in Partial Fulfillment \\ of the Requirements \\ for the Degree of
}

Doctor of Philosophy

The University of Texas at Austin

August 2013 


\section{Dedication}

To my devoted wife Leila, and my beloved parents Mohammad and Shamsi, for their endless love, patience, support, and encouragements;

To my lovely son Mahdi, a blessing in my life. 


\section{Acknowledgements}

I am sincerely grateful to my supervisor, Dr. Kamy Sepehrnoori, for his invaluable advices and excellent supervision throughout my $\mathrm{PhD}$ study. I would also like to express my deepest gratitude to my co-supervisor, Dr. Russell T. Johns, for his excellent guidance and continuous support and encouragement during this research. I am privileged to have had an opportunity to work with them. I would also like to thank other members of my dissertation committee, Dr. Mojdeh Delshad, Dr. Chun Huh, and Dr. Todd Arbogast, for their time and effort to serve on my committee and review my dissertation.

I greatly acknowledge the members of the Reservoir Simulation Joint Industry Project at the Center for Petroleum and Geosystems Engineering at The University of Texas at Austin, and the members of the Gas Flooding Research Project at the EMS Energy Institute at the Pennsylvania State University for the financial support of this research. I would also like to acknowledge the staff of the Department of Petroleum and Geosystems Engineering, Dr. Roger Terzian, Frankie Hart, Cheryl Kruzie, Michelle Mason, Glen Baum, Gary Miscoe, and Mary Pettengill for their technical and administrative support.

I would like to express my gratitude to all friends who helped and supported me throughout my PhD study. I am especially grateful to Dr. Abdoljalil Varavei for his indispensable help and guidance during this research. I thank Dr. Chowdhury Mamun who reviewed this dissertation. I am also very thankful to Javad Behseresht, Mohammad Hossein Kalaei, Mohammad Mirzaei, Ali Goudarzi, Hamid Lashgari, Aboulghasem Kazemi-Nia, Mahdy Shirdel, Saeedeh Mohebbinia, Hamed Darabi, Mahdi Haddad, 
Amir-Reza Rahmani, Hamid Hadibeik, Rohollah Abdollah Pour, Meghdad Roshanfekr, Gholamreza Garmeh, and many other friends that I have not mentioned their names.

I would like to express my deepest gratitude to my wife and my parents for their endless love, support, and encouragements throughout my graduate study at The University of Texas at Austin. My achievements would not have been possible without their help and inspiration. Finally, I would like to thank my sisters and my parents in-law, who have supported me in every possible way. 


\title{
Development of an Efficient Embedded Discrete Fracture Model for 3D Compositional Reservoir Simulation in Fractured Reservoirs
}

\author{
Ali Moinfar, Ph.D. \\ The University of Texas at Austin, 2013 \\ Supervisor: Kamy Sepehrnoori \\ Co-Supervisor: Russell T. Johns
}

\begin{abstract}
Naturally fractured reservoirs (NFRs) hold a significant amount of the world's hydrocarbon reserves. Compared to conventional reservoirs, NFRs exhibit a higher degree of heterogeneity and complexity created by fractures. The importance of fractures in production of oil and gas is not limited to naturally fractured reservoirs. The economic exploitation of unconventional reservoirs, which is increasingly a major source of shortand long-term energy in the United States, hinges in part on effective stimulation of lowpermeability rock through multi-stage hydraulic fracturing of horizontal wells. Accurate modeling and simulation of fractured media is still challenging owing to permeability anisotropies and contrasts. Non-physical abstractions inherent in conventional dual porosity and dual permeability models make these methods inadequate for solving different fluid-flow problems in fractured reservoirs. Also, recent approaches for discrete fracture modeling may require large computational times and hence the oil industry has not widely used such approaches, even though they give more accurate representations of fractured reservoirs than dual continuum models.
\end{abstract}


We developed an embedded discrete fracture model (EDFM) for an in-house fully-implicit compositional reservoir simulator. EDFM borrows the dual-medium concept from conventional dual continuum models and also incorporates the effect of each fracture explicitly. In contrast to dual continuum models, fractures have arbitrary orientations and can be oblique or vertical, honoring the complexity and heterogeneity of a typical fractured reservoir. EDFM employs a structured grid to remediate challenges associated with unstructured gridding required for other discrete fracture models. Also, the EDFM approach can be easily incorporated in existing finite difference reservoir simulators. The accuracy of the EDFM approach was confirmed by comparing the results with analytical solutions and fine-grid, explicit-fracture simulations. Comparison of our results using the EDFM approach with fine-grid simulations showed that accurate results can be achieved using moderate grid refinements. This was further verified in a mesh sensitivity study that the EDFM approach with moderate grid refinement can obtain a converged solution. Hence, EDFM offers a computationally-efficient approach for simulating fluid flow in NFRs. Furthermore, several case studies presented in this study demonstrate the applicability, robustness, and efficiency of the EDFM approach for modeling fluid flow in fractured porous media.

Another advantage of EDFM is its extensibility for various applications by incorporating different physics in the model. In order to examine the effect of pressuredependent fracture properties on production, we incorporated the dynamic behavior of fractures into EDFM by employing empirical fracture deformation models. Our simulations showed that fracture deformation, caused by effective stress changes, substantially affects pressure depletion and hydrocarbon recovery. Based on the examples presented in this study, implementation of fracture geomechanical effects in EDFM did not degrade the computational performance of EDFM. 
Many unconventional reservoirs comprise well-developed natural fracture networks with multiple orientations and complex hydraulic fracture patterns suggested by microseismic data. We developed a coupled dual continuum and discrete fracture model to efficiently simulate production from these reservoirs. Large-scale hydraulic fractures were modeled explicitly using the EDFM approach and numerous small-scale natural fractures were modeled using a dual continuum approach. The transport parameters for dual continuum modeling of numerous natural fractures were derived by upscaling the EDFM equations. Comparison of the results using the coupled model with that of using the EDFM approach to represent all natural and hydraulic fractures explicitly showed that reasonably accurate results can be obtained at much lower computational cost by using the coupled approach with moderate grid refinements. 


\section{Table of Contents}

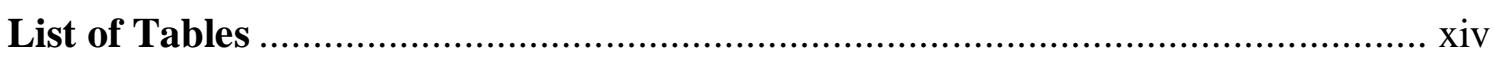

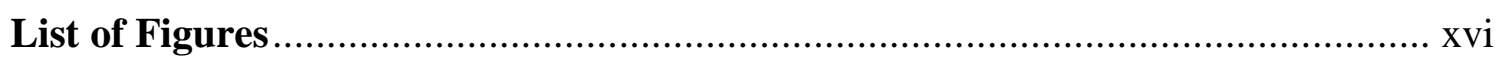

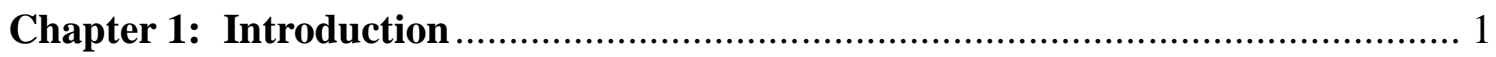

1.1 Modeling Fluid Flow in Fractured Reservoirs ........................................... 1

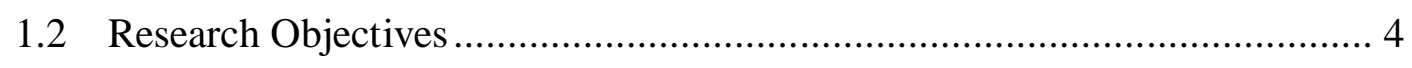

1.3 Brief Description of Chapters............................................................... 5

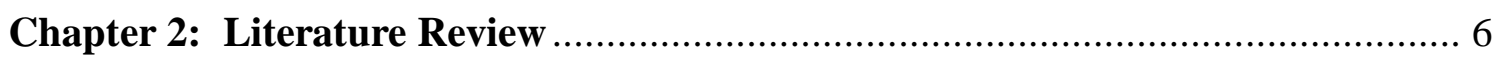

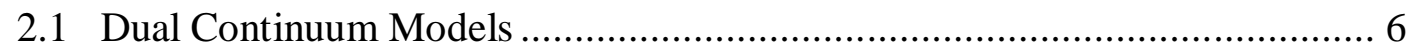

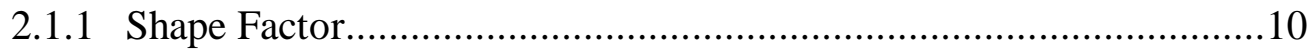

2.1.2 Improvements in Dual Porosity Simulation.......................................12

2.1.3 Warren and Root Dual Porosity Solution ..........................................17

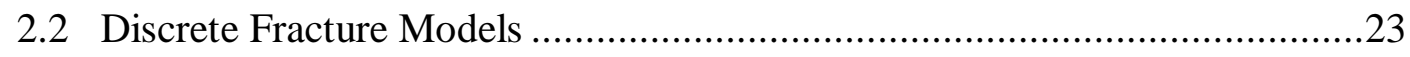

2.3 Analytical Solutions for Hydraulically Fractured Wells .................................26

2.4 Laboratory Experiments in Fractured Porous Media ....................................27

Chapter 3: General Purpose Adaptive Simulator (GPAS) ………..........................30

3.1 Overview and Framework of GPAS …………….......................................

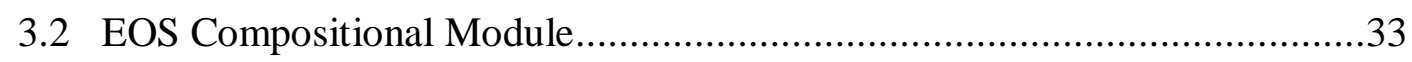

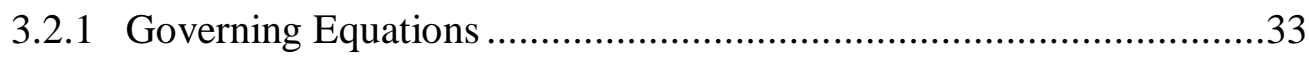

3.2.1.1 Material Balance Equations...................................................34

3.2.1.2 Phase Equilibrium Equations ................................................

3.2.1.3 Pore Volume Constraint Equation ...........................................35

3.2.2 Phase Behavior Calculations...............................................................

3.2.3 Primary Variables and Solution Procedure ………...............................39

3.3 Chemical Compositional Module .............................................................. 41

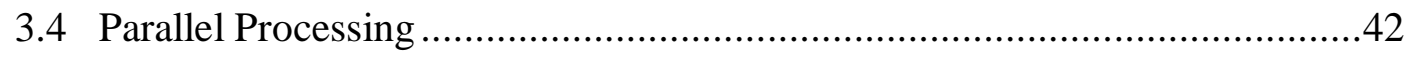


Chapter 4: Embedded Discrete Fracture Model (EDFM)

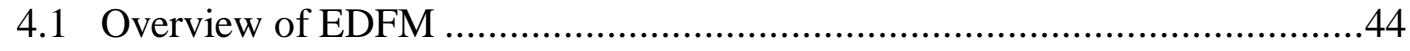

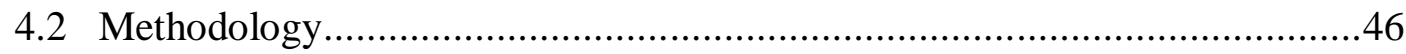

4.2.1 Fracture Discretization .......................................................47

4.2.2 Non-Neighboring Connection (NNC) ......................................52

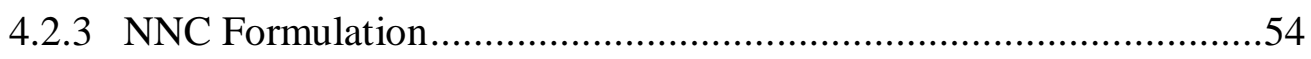

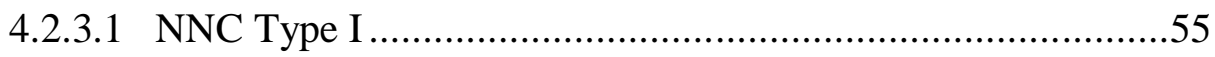

4.2.3.2 NNC Type II.....................................................57

4.2.3.3 NNC Type III .....................................................57

4.2.4 Transmissibility of Neighboring Fracture Control Volumes .............58

4.2.5 Well and Fracture Intersection ..................................................59

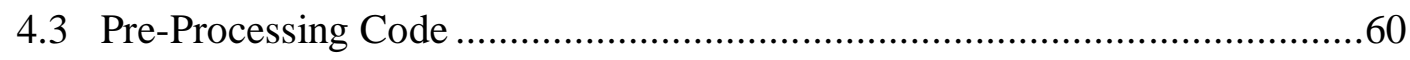

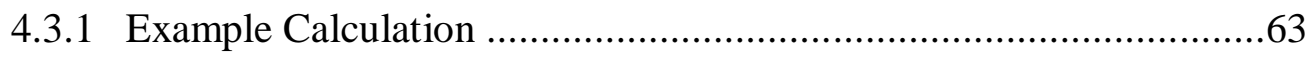

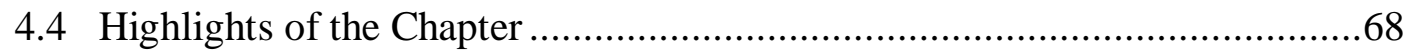

Chapter 5: Verification of Embedded Discrete Fracture Model ........................69

5.1 Comparison to Analytical Solutions ...................................................69

5.2 Comparison to Fine Grid Explicit Fracture Simulations........................... 74

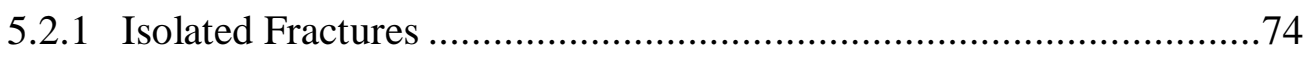

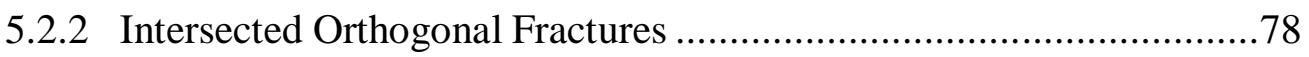

5.2.3 Non-Orthogonal Non-Aligned Fracture ....................................85

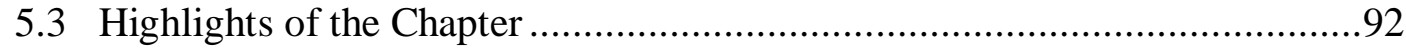

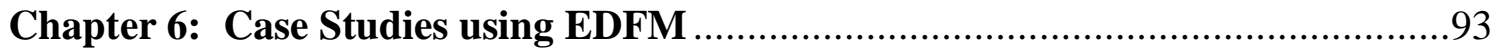

6.1 Simulation Examples Using EDFM in GPAS ................................... 94

6.1.1 2D Water-Flooding (Effect of Capillary Pressure) ........................94

6.1.2 3D Gas Injection (Effect of Gravity) ......................................... 105

6.1 .3 3D Water Flooding ........................................................ 110

6.1.4 3D Primary Depletion............................................................113

6.2 Comparison of EDFM to an Unstructured DFM and a Dual Permeability Model ................................................................................................ 117

6.2.1 Chevron Discrete Fracture Models......................................... 117 
6.2.2 Grid Sensitivity of DFMs ...........................................................119

6.2.3 Dual Permeability Modeling ..........................................................123

6.2.4 Comparison of DFMs and Dual Permeability ................................124

6.2.4.1 2D Sparsely Fractured Reservoir, Water Injection................124

6.2.4.2 3D Sparsely Fractured Reservoir, Gas-Oil Gravity Drainage126

6.2.4.3 Irregular and Anisotropic 2D Fractured Reservoir, Water

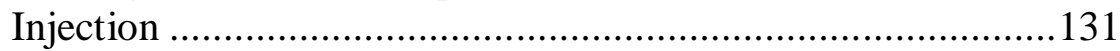

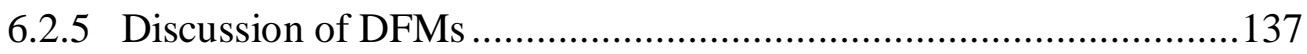

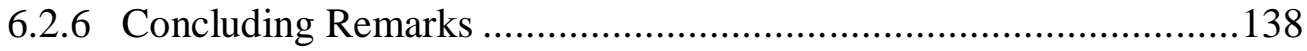

6.3 Highlights of the Chapter ..........................................................................139

Chapter 7: Incorporation of Dynamic Behavior of Fractures into EDFM ............140

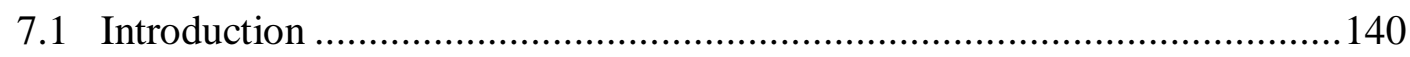

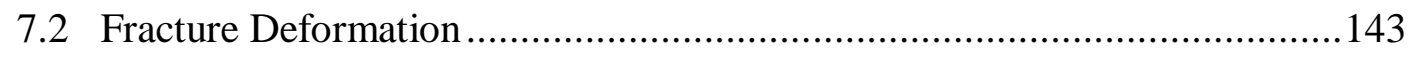

7.2.1 Deformation of Natural Fractures .................................................143

7.2.2 Deformation of Propped Hydraulic Fractures...................................148

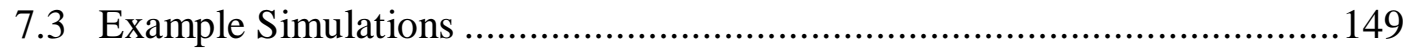

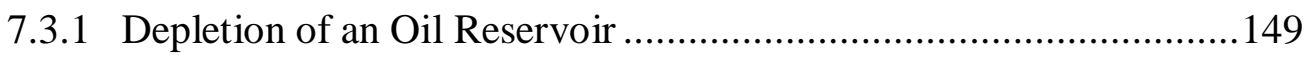

7.3.2 Depletion of an Unconventional Gas Reservoir ............................... 163

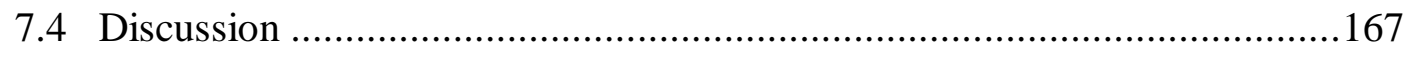

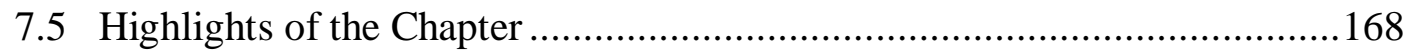

\section{Chapter 8: A Coupled Dual Continuum and Discrete Fracture Model for} Application in Unconventional Reservoirs ……….........................................169

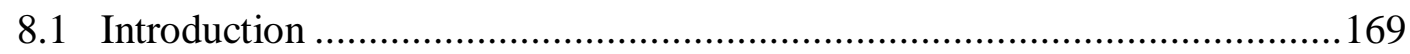

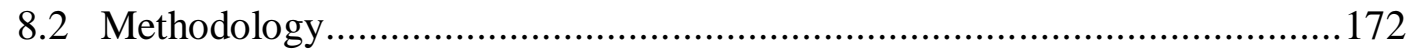

8.2.1 Transport Parameters for Dual Continuum Modeling ......................175

8.3 Accuracy and Performance of the Coupled Approach.................................178

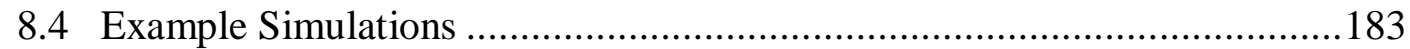

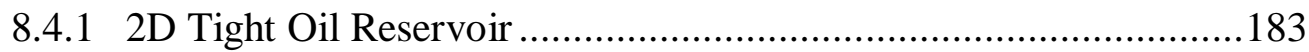

8.4.2 3D Unconventional Gas Reservoir ..................................................195

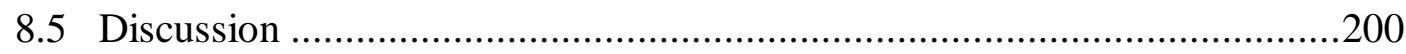




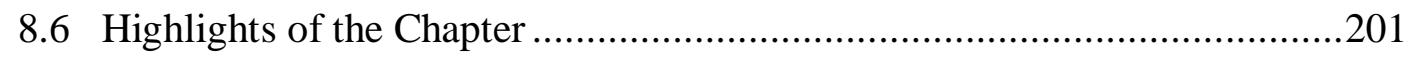

Chapter 9: Summary, Conclusions, and Recommendations ............................202

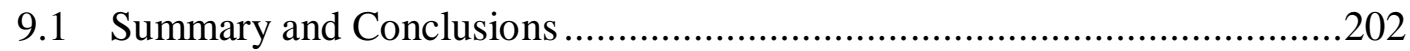

9.2 Recommendations for Future Research ..............................................206

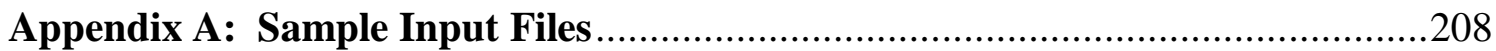

A.1 Compositional Gas Injection using EDFM (Section 5.2.2).....................208

A.2 Coupled Dual Continuum and EDFM Simulation (Section 8.4.2) ............211

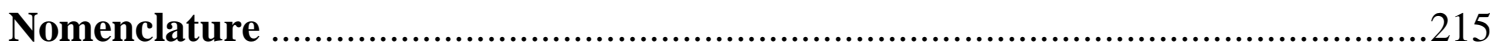

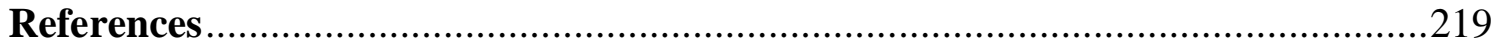




\section{List of Tables}

Table 4.1: Fracture endpoints for the model reservoir shown in Figure 4.9a...............64

Table 4.2: A portion of the calculated data for the fractures in Figure 4.9. .................66

Table 4.3: NNCs type II and type III for the reservoir shown in Figure 4.9. The cell number of NNC pairs and the transmissibility assigned to them are presented in this table.

Table 5.1: Grid description for the EDFM simulations performed for the models shown in Figures 5.1a and 5.2a.

Table 5.2: Summary of properties assumed in the simulations performed for the model reservoir shown in Figure 5.3.

Table 5.3: Properties of the hydrocarbon components used in the simulations performed for the model reservoir shown in Figure 5.6.

Table 5.4: Summary of properties used in the simulations performed for the model reservoir shown in Figure 5.6.

Table 5.5: Summary of properties used in the simulations performed for the model reservoir shown in Figure 5.12.

Table 6.1: Petrophysical properties assumed in the simulations performed for the model reservoir shown in Figure 6.10

Table 6.2: Properties of the hydrocarbon components used in the simulations performed for the model reservoir shown in Figure 6.10.

Table 6.3: Petrophysical properties and wellbore operating constraints assumed in the simulations performed for the model reservoirs shown in Figure 6.20......121 
Table 6.4: Petrophysical properties assumed in the simulations performed for the

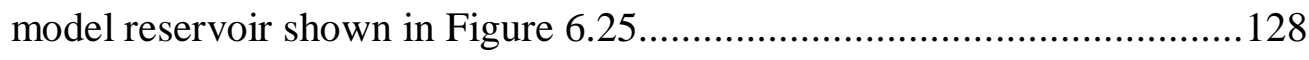

Table 7.1: Conductivity of hydraulic fractures in Figure 7.12 versus closure stress ....165

Table 8.1: Summary of properties used in the simulations performed for the model reservoir shown in Figure 8.2...................................................... 180

Table 8.2: Grid description for the simulations performed for the model shown in

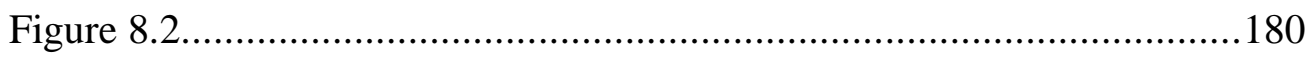

Table 8.3: Grid description for various simulations performed in Section 8.4.1........194

Table 8.4: Summary of properties used in the simulations performed for the model reservoir shown in Figure 8.13 ...................................................... 197

Table 8.5: Grid description for two simulations performed in Section 8.4.2 ..............197 


\section{List of Figures}

Figure 2.1: An idealized sugar cube representation of a fractured reservoir (from

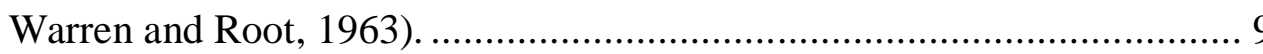

Figure 2.2: Discretization of a matrix block into a sequence of nested volume elements for the MINC method (from Wu and Pruess, 1988).....................15

Figure 2.3: Schematic of connections in a dual permeability model. The matrix-tomatrix flow depicted by dashed double-headed arrows is neglected for

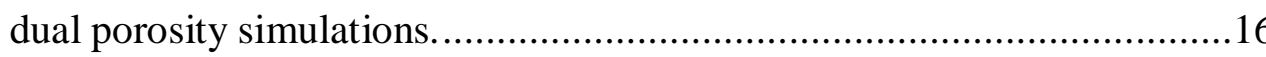

Figure 2.4: Dimensionless pressure response as a function of dimensionless time calculated by the approximate solution (Equation 2.40) and using the Stehfest method, corresponding to Figure 5 in Warren and Root (1963)...22

Figure 2.5: A 2D example of a fractured porous medium to be modeled using an unstructured control-volume finite-difference formulation. The thick line segments in the grid domain represent the fractures. One node is associated with each control volume (from Karimi-Fard et al., 2004).......25

Figure 4.1: Intersection of a vertical fracture plane and a matrix gridblock, which is always a rectangle.

Figure 4.2: Possible intersections of an inclined fracture plane and a matrix gridblock, which can be a triangle, quadrilateral, pentagon, or hexagon....48 
Figure 4.3: Geometrical representation of a fractured model $(200 \mathrm{ft} \times 200 \mathrm{ft} \times 40 \mathrm{ft})$ used to illustrate the methodology applied in EDFM. The model comprises two tilted fractures, black and red fractures, which are inclined 60 and 75 degrees from the horizontal plane, respectively. The matrix grid is $10 \times 10 \times 2$ cells in the $\mathrm{x}, \mathrm{y}$, and $\mathrm{z}$ directions, respectively, with cell dimensions of $20 \times 20 \times 20 \mathrm{ft}$ in all directions.

Figure 4.4: Specification of gridblocks containing a segment of a fracture for (a) layer 1, and (b) layer 2 in the $\mathrm{z}$ direction of the model shown in Figure 4.3. Cells containing a segment of the black fracture are marked with black circles and those containing a segment of the red fracture are marked with red squares. ............................................................50

Figure 4.5: Arrangement of fracture cells in the fracture domain for (a) layer 1, and (b) layer 2 of the model shown in Figure 4.3. Fracture cells are marked and numbered in this figure, which correspond to those in Figure 4.4. The blue dashed lines represent non-neighboring connections (NNC) for the intersection of two fractures. Also, the green dashed line represents only one example of NNC between two cells of an individual fracture. ....51 
Figure 4.6: Three types of non-neighboring connections are required in the computational domain. (a) When a fracture segment is embedded in a gridblock, there is a NNC between the fracture control volume and the matrix cell. (b) When two fracture planes intersect in a gridblock, there is a NNC between corresponding fracture control volumes. The black solid line shows the intersection line bounded in the gridblock. In a later section, this length will be used to calculate the transmissibility between two intersecting fractures. (c) When two fracture segments embedded in neighboring cells are not neighbors in the computational domain, there is a NNC between corresponding fracture control volumes. The black solid line is the intersection line of the fracture plane and the common face of two neighboring gridblocks. .................................................53

Figure 4.7: Analytical expressions of average normal distance between a matrix gridblock and an embedded fracture for some simple 2D scenarios (from

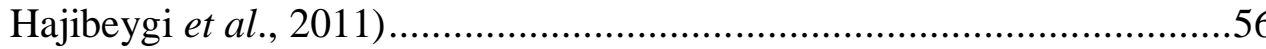

Figure 4.8: Examples of average normal distance between a matrix cell and its embedded fracture for complex 3D scenarios. (a) Normal vector of the fracture plane is $(-0.5,0.5, \sqrt{2} / 2)$ and the fracture plane passes through the point $(10,10,10)$. (b) Normal vector of the fracture plane is $(0.5,0.8,-$ $0.3317)$ and fracture plane passes through the point $(7,1,10)$

Figure 4.9: (a) A synthetic 2D model containing 14 fractures. (b) Arrangement of fracture control volumes in the fracture domain, which are marked with the same color as their corresponding fracture in Figure 4.9a. Also, blue dashed lines show the NNCs associated with intersection of fractures (NNC type II) 
Figure 5.1: (a) Geometrical configuration of a reservoir with a multiply-fractured horizontal well producing at a constant bottom-hole pressure. (b) Comparison of dimensionless rate versus dimensionless time calculated by the EDFM simulation and analytical solutions (from Chen and Raghavan, 1997).................................................................. 72

Figure 5.2: (a) Geometrical configuration of a reservoir with a multiply-fractured horizontal well producing at a constant rate. (b) Comparison of dimensionless wellbore pressure derivative versus dimensionless time calculated by the EDFM approach and analytical solutions (from Chen

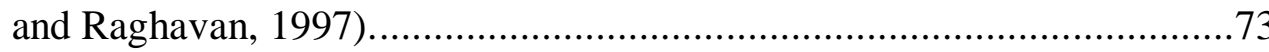

Figure 5.3: Geometrical representation of a simple 3D fractured reservoir $(600 \mathrm{ft} \times$ $300 \mathrm{ft} \times 30 \mathrm{ft}$ ) containing two isolated, discrete fractures. Both fractures are vertical and aligned with the coordinate axes.

Figure 5.4: Profiles of water saturation and pressure predicted by (a) EDFM, and (b) fine-grid explicit-fracture model at $0.26 \mathrm{PV}$ injected. The first and second rows show water saturation maps in the first and second computational layers, respectively. The third row compares the pressure maps in the reservoir.

Figure 5.5: Comparison of oil production rate over a year (0.95 PV) of water injection for the model reservoir shown in Figure 5.3, calculated by the fine-grid explicit-fracture model and EDFM.

Figure 5.6: Geometrical representation of a simple fractured reservoir $(600 \mathrm{ft} \times 300$ $\mathrm{ft} \times 15 \mathrm{ft}$ ) containing three intersected fractures. All fractures are vertical and aligned with the coordinate axes. 
Figure 5.7: Profiles of reservoir pressure predicted by (a) EDFM and (b) fine-grid explicit-fracture model after 130 days of gas injection for the model reservoir shown in Figure 5.6.

Figure 5.8: Profiles of gas saturation predicted by (a) EDFM and (b) fine-grid explicit-fracture model after 130 days of gas injection for the model reservoir shown in Figure 5.6.

Figure 5.9: Profiles of $\mathrm{CO}_{2}$ mole fraction in the oleic and gaseous phases predicted by (a) EDFM and (b) fine-grid explicit-fracture model after 130 days of gas injection for the model reservoir shown in Figure 5.6.

Figure 5.10: Comparison of (a) oil production rate, and (b) gas production rate over 600 days of gas injection for the model reservoir shown in Figure 5.6, calculated by the fine-grid explicit-fracture model and EDFM.

Figure 5.11: Profiles of gas saturation predicted by EDFM after (a) 1 month, (b) 3 months, (c) 6 months, and (d) 1 year of gas injection.

Figure 5.12: (a) A very fine stair-stepping representation of a non-aligned fracture used to investigate the accuracy of the EDFM approach for modeling arbitrary-oriented fractures. The domain dimensions are $4 \times 4 \times 1 \mathrm{ft}$. (b) An enlarged region of the model shown in Figure 5.12a, depicting the size of the gridblocks used to represent the fracture gridblocks................86

Figure 5.13: Representation of fracture permeability in the fine-grid simulation with respect to the fracture permeability in the EDFM simulations. 
Figure 5.14: Profiles of water saturation predicted by (a) fine-grid explicit-fracture model, (b) EDFM approach using a 10×10×1 matrix grid, (c) EDFM approach using a $20 \times 20 \times 1$ matrix grid, and (d) EDFM approach using a $40 \times 40 \times 1$ matrix grid after 140 days of water injection $(0.233 \mathrm{PV}$ injected)

Figure 5.15: Comparison of oil production rate over 350 days of water injection for the model reservoir shown in Figure 5.12a, calculated by the fine-grid and EDFM simulations.

Figure 6.1: A synthetic 2D fractured reservoir $(500 \mathrm{ft} \times 500 \mathrm{ft} \times 20 \mathrm{ft})$ considered in Example 1. The model contains 14 long, vertical fractures striking at different orientations.

Figure 6.2: Comparison of oil production rate calculated in the absence and presence of capillary pressure contrasts between matrix and fracture for the model reservoir shown in Figure 6.1

Figure 6.3: Water saturation profiles calculated by EDFM at (a) $0.17 \mathrm{PV}$ injected (150 days), and (b) $0.28 \mathrm{PV}$ injected (250 days). The first row shows water saturation maps simulated in the absence of capillary contrasts and the second row shows water saturation maps simulated in the presence of capillary contrasts between fractures and matrix

Figure 6.4: Comparison of oil production rate calculated by the EDFM approach using four grid resolutions under consideration for the model reservoir shown in Figure 6.1 in the absence of capillary pressure contrasts. 
Figure 6.5: Profiles of water saturation predicted by (a) EDFM using a $20 \times 20 \times 1$ matrix grid, (b) EDFM using a 40×40×1 matrix grid, (c) EDFM using a $50 \times 50 \times 1$ matrix grid, and (d) EDFM using a $80 \times 80 \times 1$ matrix grid after 250 days of water injection (0.28 PV injected) for the model reservoir shown in Figure 6.1 in the absence of capillary pressure contrasts......... 100

Figure 6.6: The top row presents an enlarged region of the reservoir shown in Figure 6.1 around a partially-penetrating fracture using (a) $40 \times 40 \times 1$ and (b) $80 \times 80 \times 1$ matrix grids. The bottom row shows the corresponding water saturation profiles after 20 days of water injection 102

Figure 6.7: Water saturation in the gridblock shown in Figure 6.6a calculated in the $40 \times 40 \times 1$ simulation compared to the average water saturation of four gridblocks shown in Figure $6.6 \mathrm{~b}$ calculated in the $80 \times 80 \times 1$ simulation. . 103

Figure 6.8: Description of (a) porosity and (b) permeability maps assigned to a $40 \times 40 \times 1$ matrix grid for the fractured reservoir shown in Figure 6.1..... 104

Figure 6.9: Water saturation profiles calculated for the heterogeneous reservoir described in Figure 6.8 at (a) 0.17 PV injected (150 days), and (b) 0.28 PV injected (250 days) 104

Figure 6.10: A synthetic 3D fractured reservoir $(500 \mathrm{ft} \times 500 \mathrm{ft} \times 40 \mathrm{ft})$ studied in the second example. The model reservoir comprises five inclined macrofractures. The dip angle of the fractures ranges from 60 to 70 degrees....106

Figure 6.11: Profiles of gas saturation in the (a) top layer, and (b) bottom layer of the model reservoir shown in Figure 6.10, simulated by EDFM. The first and second rows show gas saturation maps after 30 and 150 days of gas injection, respectively 108 
Figure 6.12: Profiles of gas saturation in the (a) top layer, and (b) bottom layer of a reservoir $(500 \mathrm{ft} \times 500 \mathrm{ft} \times 40 \mathrm{ft})$ without natural fractures. The saturation maps are presented at 150 days of gas injection...................108

Figure 6.13: Comparison of (a) oil production rate, and (b) average reservoir pressure, over a year of gas injection into the model reservoir shown in Figure 6.10 , in the presence and absence of the fracture network...........109

Figure 6.14: A synthetic 3D fractured reservoir $(500 \mathrm{ft} \times 500 \mathrm{ft} \times 80 \mathrm{ft})$ studied in the third example. The model reservoir comprises 13 inclined macrofractures. The dip angle of the fractures ranges from 55 to 90 degrees....110

Figure 6.15: Profiles of water saturation in the (a) top layer, (b) second layer, (c) third layer, and (d) bottom layer of the model reservoir shown in Figure 6.14. The saturation maps are presented at 80 days of water injection.

Figure 6.16: Oil production rate calculated by EDFM for the model reservoir shown in Figure 6.14, compared with the one calculated for the same reservoir wherein all fractures are assumed to be vertical. .112

Figure 6.17: A synthetic 3D model reservoir $(1000 \times 1000 \times 75 \mathrm{ft})$ that contains a total of 150 vertical and slanted natural fractures.

Figure 6.18: Profiles of pressure after 150 days of production in the (a) top, (b) middle, and (c) bottom numerical layers of the model reservoir shown in Figure 6.17.

Figure 6.19: History of (a) oil production rate, and (b) average reservoir pressure over two years of production for the model reservoir shown in Figure 6.17...116 
Figure 6.20: (a) A synthetic 2D $(5100 \mathrm{ft} \times 5100 \mathrm{ft})$ reservoir used to evaluate the sensitivity of DFMs to grid orientation. Panels (b) and (c) show the same fractured realization overlain on rotated coordinate axes. The coordinate $\mathrm{x}$ and $\mathrm{y}$ axes in Panels (b) and (c) are rotated $22.5^{\circ}$ and $45^{\circ}$ counterclockwise, respectively, with regard to the coordinate axes in Panel (a). The reservoir model is cylindrical with the thickness of $20 \mathrm{ft}$.

Figure 6.21: Water saturation maps simulated by EDFM for three grid orientations shown in Figure 6.20. Panels (a), (b), and (c) correspond to similar panels in Figure 6.20.

Figure 6.22: Water saturation maps simulated by USDFM for three grid orientations shown in Figure 6.20. Panels (a), (b), and (c) correspond to similar panels in Figure 6.20. The red circle in Panel (a) indicates the region where water saturation map differs slightly from those shown in Panels (b) and (c).... 122

Figure 6.23: Water saturation map simulated by the systematic dual permeability model for the sparsely fractured reservoir of Figure 6.20a.

Figure 6.24: Comparison of field water-cut calculated by dual permeability, EDFM, and USDFM models for the sparsely fractured reservoir of Figure 6.20a.125

Figure 6.25: A 3D $(5100 \mathrm{ft} \times 5100 \mathrm{ft} \times 2000 \mathrm{ft})$ sparsely fractured reservoir studied in the second comparison case. A horizontal producer is completed at the bottom of reservoir in a high-permeability layer to provide productivity during depletion. The horizontal producer intersects some discrete fractures. 
Figure 6.26: Cut-section view of profiles of pressure after 5 years of production simulated by (a) USDFM, (b) EDFM, and (c) dual permeability models for the fractured reservoir shown in Figure 6.25.

Figure 6.27: Cut-section view of profiles of oil saturation after 5 years of production simulated by (a) USDFM, (b) EDFM, and (c) dual permeability models for the fractured reservoir shown in Figure 6.25. Panel (a) also shows the oil saturation in some fractures

Figure 6.28: Comparison of (a) oil production-rate and (b) cumulative oil produced, calculated by USDFM, EDFM, and dual permeability models for the fractured reservoir shown in Figure 6.25.

Figure 6.29: A synthetic $2 \mathrm{D}(5100 \mathrm{ft} \times 5100 \mathrm{ft})$ complex fractured reservoir studied in the third comparison example. This model comprises 254 fractures in two nearly orthogonal sets. The reservoir thickness is $20 \mathrm{ft}$.

Figure 6.30: Comparison of water-cut calculated by USDFM approach in the presence and absence of fractures. In the presence of fractures, Producer 3 records the earliest water breakthrough time among producing wells while the latest breakthrough occurs at Producer 1.

Figure 6.31: Profiles of water saturation after (a) 1616 and (b) 5356 days of water injection acquired in the fractured reservoir shown in Figure 6.29. The first, second, third, and forth rows correspond to simulations of dual permeability, USDFM, fine-grid $(85 \times 85)$ EDFM, and coarse-grid (51×51) EDFM approaches, respectively. 
Figure 6.32: Comparison of (a) field water-cut, (b) water-cut of Producer 3 which has the earliest breakthrough time, and (c) water-cut of Producer 1 which has the latest breakthrough time, calculated by EDFM, USDFM, and dual permeability models for the fractured reservoir shown in Figure 6.29

Figure 6.33: Comparison of field water-cut, calculated by fine-grid $(85 \times 85)$ and coarse-grid $(51 \times 51)$ EDFM simulations for the fractured reservoir shown in Figure 6.29. 136

Figure 7.1: Normal stress $\left(\sigma_{n}\right)$ vs. closure $\left(\Delta V_{j}\right)$ curves for a range of (a) fresh joints and (b) weathered joints in different rock types, under repeated loading cycles (from Bandis et al., 1983).

Figure 7.2: A synthetic $2 \mathrm{D}$ fractured reservoir $(1000 \times 1000 \times 50 \mathrm{ft})$ considered in the first example. Three fractures are shown with numbers for detailed investigation during production. 150

Figure 7.3: Ratio of fracture aperture to the maximum closure calculated by the Barton-Bandis model. Initial normal stiffness is $7 \times 10^{4} \mathrm{psi} / \mathrm{ft}$ and maximum fracture closure is $0.011 \mathrm{ft}$.

Figure 7.4: Comparison of (a) pressure and (b) permeability profiles simulated for three fractures, shown with numbers in Figure 7.2, over two years of production.

Figure 7.5: Pressure profiles after 150 days of production simulated by the coupled model when (a) fracture properties are pressure-dependent, and (b) fracture properties are constant during production. 
Figure 7.6: History of (a) oil production rate, and (b) cumulative oil production over two years of production, for the dynamic and static treatments of fractures in the simulations. 156

Figure 7.7: Ratio of fracture aperture to the maximum closure calculated by the Barton-Bandis model. Initial normal stiffness is $3 \times 10^{5} \mathrm{psi} / \mathrm{ft}$ and maximum fracture closure is $0.011 \mathrm{ft}$.

Figure 7.8: History of cumulative oil production over two years of production for the dynamic and static treatments of fractures in the simulations. 158

Figure 7.9: A synthetic 3D model reservoir $(1000 \times 1000 \times 75 \mathrm{ft})$ that contains a total of 148 vertical and slanted natural fractures.

Figure 7.10: Profiles of pressure after 150 days of production for the (a) dynamic and (b) static treatments of fractures. The first, second, and third rows correspond to the top, middle, and bottom layers of the reservoir shown in Figure 7.9

Figure 7.11: History of (a) cumulative oil production, and (b) average reservoir pressure over two years of production from the model reservoir shown in Figure 7.9. Comparisons are made between the dynamic and static treatments of fractures. 162

Figure 7.12: A 2D model representing an unconventional gas reservoir, which is considered in the second example. The model comprises a $2400-\mathrm{ft}$ horizontal well (brown line) and five transverse hydraulic fractures (blue planes).

Figure 7.13: Pressure profiles after 15 years of production when fracture properties are pressure-dependent and formation permeability is (a) $0.0001 \mathrm{md}$, and (b) $0.001 \mathrm{md}$. 
Figure 7.14: Comparison of the cumulative oil production over 30 years for the dynamic and static treatments of hydraulic fractures when formation permeability is (a) $0.0001 \mathrm{md}$, and (b) $0.001 \mathrm{md}$. 166

Figure 8.1: (a) A synthetic 2D reservoir used to illustrate the methodology applied in the coupled model. The model comprises a 2000-ft horizontal well (brown line), 7 transverse hydraulic fractures (blue lines), and 1819 small-scale natural fractures (black lines). (b) An enlarged region of the model in Figure 8.1a showing the grid boundaries and the embedded natural fractures.

Figure 8.2: A synthetic 2D reservoir used to evaluate the accuracy of the coupled approach. The model comprises a 660-ft horizontal well (brown line), four transverse hydraulic fractures (blue lines), and 740 small-scale natural fractures (black lines).

Figure 8.3: Pressure profiles simulated by the (a) EDFM approach, (b) coupled model using a $100 \times 67 \times 1$ matrix grid, (c) coupled model using a $60 \times 41 \times 1$ matrix grid, and (d) coupled model using a $30 \times 21 \times 1$ matrix grid after 200 days of production for the reservoir shown in Figure 8.2. .181

Figure 8.4: Comparison of oil production rate over six years of production for the reservoir shown in Figure 8.2 calculated by the EDFM approach and three simulations using the coupled approach. 182

Figure 8.5: Pressure profiles simulated by the coupled model after (a) 3 months, (b) 1 year, (c) 3 years, and (d) 6 years of production for the reservoir shown in Figure 8.1a. 185 
Figure 8.6: Pressure profiles simulated by EDFM after (a) 3 months, (b) 1 year, (c) 3 years, and (d) 6 years of production for the reservoir shown in Figure 8.1a but in the absence of the natural fracture network. 186

Figure 8.7: Comparison of (a) oil production rate, and (b) average reservoir pressure, over six years of production for the reservoir shown in Figure 8.1a, in the presence and absence of the natural fracture network.

Figure 8.8: (a) A model reservoir which retains the natural fracture network in Figure 8.1a, but contains longer hydraulic fractures. (b) Pressure profile simulated by the coupled model after three years of production for the reservoir shown in Figure 8.8a.

Figure 8.9: (a) A model reservoir which retains the natural fracture network in Figure 8.1a, but contains ten hydraulic fractures. (b) Pressure profile simulated by the coupled model after three years of production for the reservoir shown in Figure 8.9a.

Figure 8.10: Comparison of (a) oil production rate, and (b) cumulative oil production, over six years of production for the reservoir models shown in Figures 8.1a, 8.8a, and 8.9a

Figure 8.11: (a) A model reservoir which retains the natural fracture network in Figure 8.1a, but comprises a multi-stranded complex hydraulic fracture pattern. (b) Schematic geometry of hydraulic fracture propagation in unconventional reservoirs (from the website of University of Michigan).193

Figure 8.12: Pressure profiles after (a) 3 months, and (b) 3 years of production for the model reservoir shown in Figure 8.11a. The first and second rows show the pressure maps simulated in the absence and presence of the natural fracture network, respectively. 
Figure 8.13: A synthetic 3D reservoir studied in Section 8.4.2. The model comprises a 1200-ft horizontal well (green line), 3 hydraulic fractures (blue planes), and 1892 small-scale natural fractures (red planes).................196

Figure 8.14: Pressure profiles simulated by the coupled model after 10 years of production in the (a) first, (b) second, (c) fourth, and (d) fifth computational layers of the model reservoir shown in Figure 8.13.........198

Figure 8.15: Gas production rate calculated by the coupled model over ten years of production for the reservoir shown in Figure 8.13 wherein all hydraulic fractures penetrate $60 \%$ of the formation height, compared with the one calculated for the same reservoir where all hydraulic fractures fully penetrate the formation height. 


\section{Chapter 1: Introduction}

\subsection{MODELing Fluid Flow in FraCtURED RESERVOIRS}

Naturally fractured reservoirs (NFRs) hold a significant amount of the world's hydrocarbon reserves. Compared to conventional reservoirs, NFRs exhibit a higher degree of heterogeneity and complexity created by fractures. From the standpoint of flow behavior, NFRs comprise two mediums, rock matrix and fractures, with drastically different properties. Generally the rock matrix provides the primary storage of hydrocarbons while the fractures serve as highly conductive flow paths. Fracture apertures are very small compared to matrix dimensions. Hence, fractures hold very little fluid, yet their permeability can be very high (e.g., hundreds of darcies).

Many naturally fractured reservoirs have depleted significantly and improved oil recovery (IOR) processes are necessary for their further development. It is well established that when IOR strategies are pursued for NFRs, injected fluids mostly flow through high-permeability fracture networks, bypassing oil in the rock matrix. Thus, extensive laboratory experiments have been conducted to investigate the efficiency and applicability of different IOR processes in fractured media (Horie et al., 1990; PooladiDarvish and Firoozabadi, 2000; Babadagli, 2001; Hirasaki and Zhang, 2004; Darvish et al., 2006). Although some experiments have yielded promising results, predictive simulations of such complex processes are required to reliably scale up the process from laboratory to field conditions.

The importance of fractures in production of oil and gas is not limited to naturally fractured reservoirs. The exploitation of unconventional reservoirs is 
increasingly a major source of short- and long-term energy in the United States. The economic development of unconventional oil and gas hinges in part on effective stimulation of low-permeability rock through multi-stage hydraulic fracturing of horizontal wells. To achieve this goal, accurate characterization and simulation of production is necessary for selecting the best stimulation strategy.

Presence of highly conductive fracture pathways at various length scales, coupled with small fracture volumes, makes numerical simulation of fluid flow in fractured reservoirs very challenging. Several approaches have been proposed to model NFRs, which can be categorized into two classes of models, dual continuum and discrete fracture models.

Presently, dual porosity and dual permeability models are the most commonly used modeling approaches for NFRs in the petroleum industry. The dual continuum model, although very efficient, is a very simplistic representation of complex NFRs. Nonphysical abstractions inherent in conventional dual porosity and dual permeability models make them inadequate for solving different fluid-flow problems in fractured reservoirs. Dual continuum models are especially appropriate for reservoirs with a large number of highly connected, small-scale fractures.

Discrete fracture models (DFMs), however, are a new class of models for simulating NFRs and have received considerable attention in the last decade. To the best of our knowledge, DFMs have not been widely used in the industry for field-scale reservoir simulation studies, even though they give more accurate representations of flow in fractured reservoirs than conventional methods. In general, when using DFMs, a large number of small gridblocks is required near the fractures, which results in increased computational time. Also, most DFM approaches require generating an unstructured grid 
to conform to the complexity of the fractures assigned to the domain of interest. Generation of such a grid for an arbitrary fracture network can be a substantial challenge.

To take advantage of both dual continuum and discrete fracture models, Lee et al. (2000, 2001) and Li and Lee (2008) presented an approach for simulating fluid flow in NFRs called the embedded discrete fracture model (EDFM). The model borrows the dual-medium concept from dual continuum models and also incorporates the effect of each fracture explicitly. EDFM uses a structured grid to represent the matrix and introduces additional fracture control volumes by computing the intersection of fractures with the matrix grid. Therefore, challenges associated with unstructured gridding are bypassed entirely. For simplicity of geometric design, Lee et al. $(2000,2001)$ and Li and Lee (2008) implemented the mentioned approach only for vertical fractures. However, field characterization studies have shown that the occurrence of obliquely dipping fractures is quite common in NFRs (Walsh and Watterson, 1988; Angerer et al., 2002; Grechka and Tsuankin, 2004). Therefore, an extended EDFM approach that considers realistic 3D discrete fractured media, including slanted fractures, is needed for modeling NFRs in a robust and efficient manner.

Pore pressure changes caused by production from a reservoir or injection into a reservoir can induce rock deformations. Fluid-flow characteristics of reservoir rocks, such as permeability and pore compressibility, can be very sensitive to effective stress changes caused by changes in pore pressure. The effect of geomechanics on fluid flow is more crucial in fractured reservoirs due to presence of fissures, which might be more stress-sensitive than the rock matrix. The flow characteristics of fractures are significantly affected by effective normal stress exerted on the fractures. In spite of extensive experimental and field studies demonstrating the dynamic behavior of fractures (Bandis et al., 1983; Barton et al., 1985; Lorenz, 1999), fracture properties have often 
been treated as static parameters in the simulations of naturally fractured reservoirs. Hence, more realistic modeling of production in fractured systems requires inclusion of the dynamic behavior of fractures in a fracture model.

Challenges for the simulation of unconventional reservoirs are not limited to the large contrast between permeability of hydraulic fractures and their neighboring tight rock matrix. Many unconventional reservoirs comprise well-developed natural fracture networks with multiple orientations and complex hydraulic fracture patterns, suggested by microseismic data. Although recent advances in seismic technology have improved mapping of fracture orientations and densities in unconventional reservoirs, detailed characterization of pre-existing natural fractures is often unavailable. Another substantial challenge for unconventional reservoir simulations is the presence of complex hydraulic fracture geometry. Conventional dual porosity and dual permeability models are not adequate for modeling these complex networks of natural and hydraulic fractures. Also, it is neither practical nor advantageous to model a large number of pre-existing fractures with a discrete fracture model. Therefore, an appropriate approach to model production from these reservoirs is to couple a DFM, accounting for the large-scale hydraulic fractures, with a dual continuum model that accounts for flow in the naturally fractured networks.

\subsection{RESEARCH OBJECTIVES}

Based on the problems described above, the main objectives of this research are as follows:

1. Implement the EDFM approach to an in-house, fully implicit, compositional reservoir simulator, called the General Purpose Adaptive Simulator (GPAS). 
The developed approach allows for compositional simulations of complex displacement processes (such as miscible gas injection) in fractured reservoirs in a robust and efficient manner.

2. Extend the EDFM method for modeling slanted fractures. Hence, the developed model could allow for multiple sets of fractures with any arbitrary orientations.

3. Incorporate the dynamic behavior of fractures into EDFM.

4. Develop a coupled dual continuum and discrete fracture model for the simulation of unconventional reservoirs that feature complex hydraulic fracture networks and numerous small-scale natural fractures.

\subsection{BRIEF DESCRIPTION OF CHAPTERS}

In Chapter 2, we present a literature review of several approaches proposed to model fluid flow in fractured reservoirs. Chapter 3 reviews the general features and formulation of GPAS. In Chapter 4, we describe the methodology used for modeling vertical and slanted fractures using the EDFM approach. The accuracy of the EDFM approach is confirmed in Chapter 5 by comparing the EDFM results with analytical solutions and fine-grid explicit-fracture simulations. We present several examples in Chapter 6 to demonstrate the applicability and performance of the EDFM approach for simulating NFRs. In Chapter 7, we present the methodology used to incorporate dynamic behavior of fractures into EDFM, followed by several example simulations. Chapter 8 presents a coupled dual continuum and discrete fracture model for application in unconventional reservoirs. Finally, Chapter 9 presents the conclusions of this research and recommendations for future research. 


\section{Chapter 2: Literature Review}

Presence of highly conductive fracture pathways at various length scales, coupled with small fracture volumes, makes numerical simulation of fluid flow in naturally fractured reservoirs (NFRs) very challenging. Several approaches have been proposed to model NFRs, which can be categorized into two classes of models, dual continuum and discrete fracture models. Presently, dual continuum models are the most commonly used modeling approach for NFRs in the petroleum industry. However, discrete fracture modeling approaches are gaining considerable interest.

\subsection{Dual ContinuUm Models}

Dual continuum models, widely used in the industry, are the conventional method for simulating NFRs. The method is based on a concept originally proposed by Barenblat et al. (1960). Subsequently, Warren and Root (1963) introduced the dual porosity model to the petroleum literature. The dual porosity model, which is also known as a sugar cube model, was first used for modeling single-phase flow in NFRs. In this model, rectilinear prisms of rock matrix are separated by an orthorhombic continuum of fractures. Dual porosity simulation involves discretization of the reservoir into two domains, matrix and fracture. Hence, every point in the reservoir contains fracture and matrix pressures and saturations. A dual porosity model presumes that the flow occurs from the matrix to the fractures, and then to the production wells. The rock matrix is where the majority of oil is stored. In the dual porosity model, matrix and fracture domains are linked to each other through an exchange term that connects each fracture cell to its corresponding matrix cell 
in a gridblock. The matrix-fracture exchange rate is controlled by a shape factor. Also, the matrix blocks in the dual porosity approach are assumed to be isotropic and homogeneous. Figure 2.1 shows an idealized sugar cube representation of a fractured reservoir, wherein highly interconnected orthogonal fractures are fed by numerous matrix blocks.

Considering single-phase fluid flow, the mass conservation equations for the fracture and matrix domains in the dual porosity approach can be expressed for the fracture domain as

$$
\frac{k_{f x}}{\mu} \frac{\partial^{2} P_{f}}{\partial x^{2}}+\frac{k_{f y}}{\mu} \frac{\partial^{2} P_{f}}{\partial y^{2}}+\frac{k_{f z}}{\mu} \frac{\partial^{2} P_{f}}{\partial z^{2}}-q_{m f}=\phi_{f} C_{f} \frac{\partial P_{f}}{\partial t}
$$

and for the matrix domain as

$$
\phi_{m} C_{m} \frac{\partial P_{m}}{\partial t}=q_{m f}
$$

The matrix-fracture transfer is represented by the pseudo-steady state relation:

$$
q_{m f}=\frac{\sigma k_{m}}{\mu}\left(P_{m}-P_{f}\right)
$$

where $k, P, \mu, C, \phi, \sigma$, and $q_{m f}$ denote permeability, pressure, fluid viscosity, total compressibility, porosity, shape factor, and matrix-fracture flow rate per unit bulk volume, respectively. The subscripts $m$ and $f$ refer to the properties in the matrix and fracture domains. The shape factor, which has the dimension of reciprocal area, reflects the geometry of matrix blocks and controls the interporosity flow between matrix and fracture domains.

Warren and Root showed that the pressure transient response of a well producing from a fractured reservoir can be characterized by two parallel semi-log straight lines, which form the early- and the late-time portions of this semi-log plot. The first straight 
line period corresponds to transient flow in the fracture system alone, while the second one is a response to transient flow in the total reservoir (matrix and fractures). A transitional curve connects the parallel segments in the pressure transient plot.

Odeh (1965) reported that often, the first straight line period on the semi-log plot and the transition zone occur rapidly and therefore, the pressure behavior of a fractured reservoir cannot always be distinguished from that of non-fractured reservoirs. Wellbore storage may also obscure this early time period associated with the fracture response. Kazemi (1969) developed a single-phase dual porosity numerical model for a 2D radial system and presented results similar to those obtained by Warren and Root (1963). Furthermore, he included a direct flux from matrix to well in the dual porosity model and showed that the direct flow from matrix to the well does not remarkably affect the pressure transient behavior of NFRs. Kazemi (1969), De Swaan (1976), and Najurieta (1980) implemented dual porosity models considering transient fluid transfer between matrix and fracture. Using these models, the pressure transient plot exhibits three semi$\log$ straight lines. The first and the third lines are parallel and correspond to the fractureonly and total reservoir responses, respectively. The slope of the second semi-log straight line, which appears in the transition zone, is one half of the slope of the first and third lines. De Swaan (1976) also provided solutions for reservoirs in which the matrix blocks are either slabs or spheres. Mavor and Cinco-Ley (1979) and Moench (1984) extended the dual porosity approach to include the effect of skin between the matrix and the fracture system. They showed that the pseudo-steady state assumption used in the transfer function of the Warren and Root's approach can be justified when the effect of skin is pronounced.

For single-phase flow the matrix block size controls the transition from early production from fractures to late production from the total reservoir. The original dual 
porosity model assumes that fracture intensity is uniform throughout the reservoir and hence the matrix block size is constant. However, geological studies of naturally fractured reservoirs in conjunction with outcrop observations indicate occurrences of non-uniform fracture patterns owing to varying lithology, bed thickness, and stress environment. Hence, some research extended dual porosity models to include variable matrix block sizes in order to make these models more realistic. Cinco-Ley et al. (1985) developed a model to investigate pressure transient response for a naturally fractured reservoir containing different matrix block sizes. They showed that the transition zone is significantly affected by matrix block size, while the early- and the late-time responses are not. Later, Belani and Jalali-Yazdi (1988), Johns and Jalali-Yazdi (1991), and Spivey and Lee (2000) examined continuous probability density functions for matrix block size to improve well-test analysis of dual porosity systems.
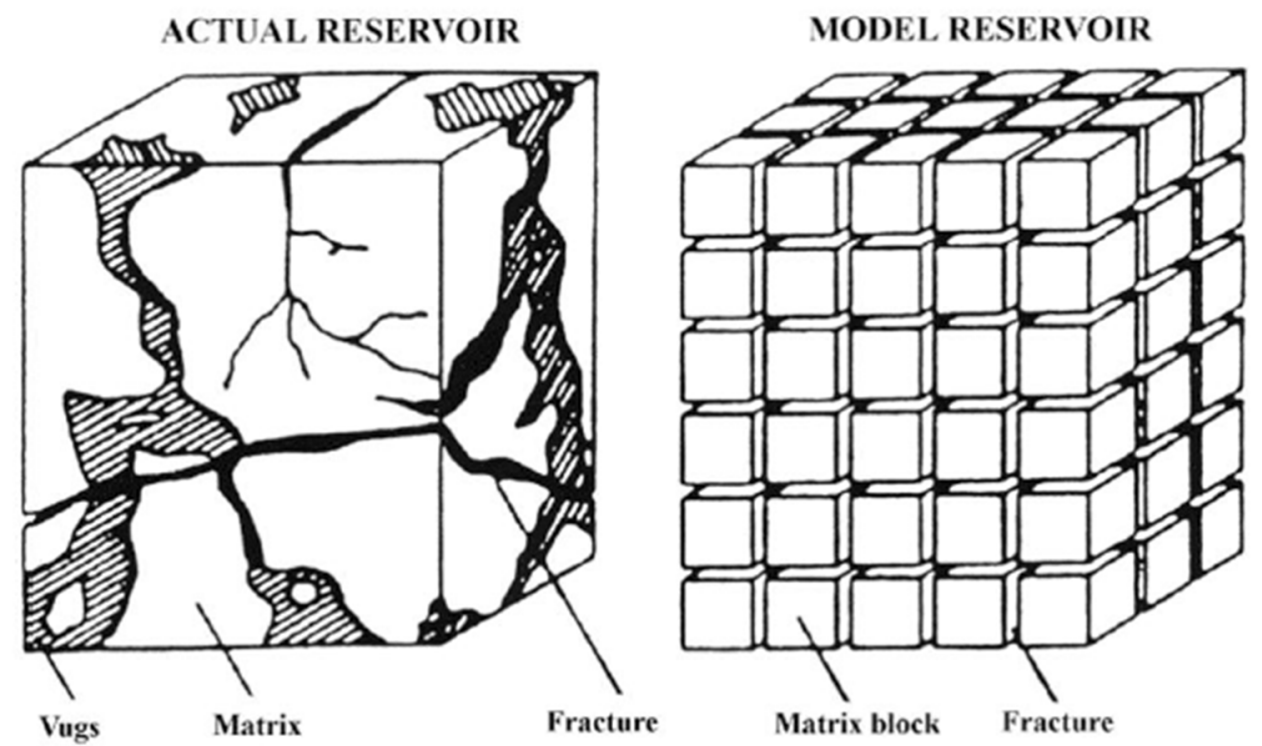

Figure 2.1: An idealized sugar cube representation of a fractured reservoir (from Warren and Root, 1963). 
Kazemi et al. (1976) and Rossen (1977) extended the Warren and Root approach to multiphase flow and developed dual porosity simulators for modeling multiphase flow in NFRs. Since then, the dual porosity approach has been implemented in many reservoir simulators for field-scale NFR simulations.

\subsubsection{Shape Factor}

The matrix-fracture exchange is a critical component of any model used for the simulation of NFRs. In the Warren and Root approach, the matrix-fracture fluid transfer is assumed to take place under pseudo-steady state conditions. As described in Equation 2.3, the matrix-fracture transfer function is proportional to a geometrical shape factor $(\sigma)$, and the driving force is the pressure difference between a matrix block and its surrounding fracture. Determination of shape factor is not a simple task because of the potential for complex interaction between fractures and matrix rock of various shapes.

Originally, Warren and Root (1963) defined the shape factor as a parameter that depends on the geometry of matrix blocks as given below:

$$
\sigma=\frac{4 n(n+2)}{L^{2}}
$$

where $n$ is the number of normal sets of fractures $(n=1,2,3)$ and $L$ is the characteristic length of matrix blocks given by

$$
\begin{array}{ll}
L=a, & \text { for } \mathrm{n}=1 \\
L=\frac{2 a b}{a+b}, & \text { for } \mathrm{n}=2 \\
L=\frac{3 a b c}{a b+a c+b c}, & \text { for } \mathrm{n}=3
\end{array}
$$

where $a, b$, and $c$ are the lengths of the block faces. 
Kazemi et al. (1976) used a finite-difference formulation for the flow between matrix and fractures and showed that for a three-dimensional case

$$
\sigma=4\left(\frac{1}{L_{x}^{2}}+\frac{1}{L_{y}^{2}}+\frac{1}{L_{z}^{2}}\right),
$$

where $L_{x}, L_{y}$, and $L_{z}$ are the distances between fractures in the $\mathrm{x}, \mathrm{y}$, and $\mathrm{z}$ directions, respectively. The shape factor proposed by Kazemi et al. (1976) has been used in a number of reservoir simulators. Coats (1989) included pseudo-steady state matrixfracture diffusion in the derivation of the matrix-fracture transfer function and obtained a shape factor exactly twice that of Kazemi et al. (1976).

These shape factors assume that the pseudo-steady state assumption is valid. Lim and Aziz (1995) considered the physics of pressure diffusion from the matrix to the fracture and presented a new shape factor as follows:

$$
\sigma=\pi^{2}\left(\frac{1}{L_{x}^{2}}+\frac{1}{L_{y}^{2}}+\frac{1}{L_{z}^{2}}\right) .
$$

They performed simulations to investigate the accuracy of various shape factors using fine-grid simulations. The results showed that their shape factor in Equation 2.7 matches with the results of fine-grid single porosity better, indicating that the pseudo-steady state assumption is not a suitable one and the pressure gradients in the matrix should be taken into account for the calculation of shape factors. They also showed that the dual porosity simulation using the Warren and Root's shape factor overestimates the recovery, while the simulation using the Kazemi's shape factor underestimates the recovery. 


\subsubsection{Improvements in Dual Porosity Simulation}

After Warren and Root who introduced the concept of dual porosity for modeling NFRs, many studies challenged the adequacy of this approach for simulating gravity segregation, capillary imbibition, and other transient phenomena that matter in individual matrix blocks. Hence, considerable effort was devoted to make the original dual porosity model more realistic. Some important improvements include: the subdomain method (Saidi, 1983; Gilman, 1986), the MINC (multiple interacting continua) method (Pruess and Narasimhan, 1985), and pseudo capillary pressure and relative permeability techniques (Thomas et al., 1983; Dean and Lo, 1988; Rossen and Shen, 1989).

A drawback of the original dual porosity model was the consideration of gravity effects. In this model, the same depth was assumed for a matrix block and its surrounding fracture. Reiss (1980) was among the first who discussed the effect of gravity on fluid transfer between matrix and fracture. Gilman and Kazemi (1983) added a gravity term to the dual porosity transfer function. The gravity term was a function of fluid contact heights in the matrix block and its surrounding fracture. Litvak et al. (1985) and Sonier et al. (1988) used similar approaches, but improved the calculation of the fluid contact heights by including irreducible saturations.

Thomas et al. (1983) developed a 3D, three-phase, finite difference dual porosity model for simulating NFRs. In order to account for the gravity effects, they introduced pseudo capillary pressure for the matrix. Dean and Lo (1988) showed that the effect of gravity segregation could be included in pseudo capillary pressure terms for both matrix and fracture. They used fine-grid simulations to determine these pseudo capillary pressure curves. Likewise, Rossen and Shen (1989) proposed a model to calculate the matrix-fracture exchange term using pseudo capillary pressure curves for both matrix and fracture. The matrix pseudo capillary pressure curve was obtained from fine-grid 
simulation of a single matrix block surrounded by fractures. Beckner et al. (1988) also used a similar approach to reproduce the experimental data of Kleppe and Morse (1974). Furthermore, Beckner et al. (1988) and Gilman and Kazemi (1988) argued that the timedependent nature of gravity segregation should be included in NFR simulations. However, one drawback of pseudo capillary pressure techniques is the time and effort required to history match the results of fine-grid simulations to generate pseudo capillary pressure curves. Rossen and Shen (1989) described a procedure for generating the pseudo curves from a single fine-grid simulation without history matching.

In the Warren and Root dual porosity approach, all matrix blocks in each computational gridblock are lumped into one source/sink term connected to a fracture. Therefore, average properties, such as average pressure and saturation, are used for the entire matrix block, resulting in an inaccurate pressure gradient between fracture and matrix. Subgridding the matrix block, which was first introduced by Saidi (1983), was a significant improvement to model the transient flow in the matrix blocks. In order to represent pressure and saturation variations more accurately, he suggested dividing a matrix block into subdomains. Saidi (1983) discretized the matrix blocks in the vertical and radial directions in a three-phase dual porosity simulator. Gilman (1986), Chen et al. (1987), Lee and Tan (1987), and Beckner et al. (1991) used similar approaches to improve the matrix representation in dual porosity simulations. Matrix block subdivision gave better resolution of pressure and saturation gradients, but significantly increased the computational cost of dual porosity simulations.

Pruess and Narasimhan (1985) introduced another dual porosity approach called the multiple interacting continua (MINC) method. Since the variations of thermodynamics conditions are much smaller in the fracture direction than perpendicular to it, they assumed that surfaces with equal distances from the fracture have the same 
flow potential. Hence, they discretized the matrix blocks into a sequence of nested volume elements such that all interfaces between volume elements are parallel to the nearest fracture, as schematically shown in Figure 2.2. Gilman (1986) developed a dual porosity simulator based on the MINC method. He divided each matrix block into rectangular rings and vertical subdomains. Hence, his model also had the same advantages as Saidi's model in modeling gravity segregation. Wu and Pruess (1988) compared the MINC method with fine-grid simulations for modeling water-oil capillary imbibition in NFRs. They showed that the MINC method predicts the water imbibition from a fracture to a matrix block more accurately than the standard dual porosity approach.

In the dual porosity approach, fluid transfer between computational matrix blocks is assumed to be negligible. This assumption is not appropriate when the matrix blocks are larger than the computational gridblocks. Thus, Blaskovich et al. (1983), Hill and Thomas (1985), and Dean and Lo (1988) developed dual permeability models that used the same approach as dual porosity models, but were augmented by matrix-to-matrix flow. Figure 2.3 schematically describes their connections in a dual permeability model. The matrix-to-matrix flow depicted by dashed double-headed arrows in Figure 2.3 is neglected for dual porosity simulations. Similar to the dual porosity model, the dual permeability model has been implemented in many reservoir simulators for field-scale simulations of NFRs. The mass conservation equation for the matrix in a dual permeability model is different from that of a dual porosity model (Equation 2.2) and is given by

$$
\phi_{m} C_{m} \frac{\partial P_{m}}{\partial t}=q_{m f}+\frac{K_{m x}}{\mu} \frac{\partial^{2} P_{m}}{\partial x^{2}}+\frac{K_{m y}}{\mu} \frac{\partial^{2} P_{m}}{\partial y^{2}}+\frac{K_{m z}}{\mu} \frac{\partial^{2} P_{m}}{\partial z^{2}} .
$$


The dual porosity approach has been implemented in various reservoir simulators at The University of Texas at Austin. Chen (1993) developed a dual porosity simulator, UTDUAL, for modeling water-flooding in fractured reservoirs. He decoupled the matrix pressure equation from the fracture pressure equation to reduce computational time. Chen (1993) studied countercurrent imbibition processes in naturally fractured reservoirs. Aldejain (1999) implemented a dual porosity model in a chemical flooding simulator (UTCHEM). Naimi-Tajdar et al. (2007) implemented the MINC method into a compositional General Purpose Adaptive Simulator (GPAS). Later, Tarahhom (2008) incorporated the fracture full-tensor permeability into GPAS.

Balogun et al. (2007), Ramirez et al. (2009), and Al-Kobaisi et al. (2009) compared dual porosity simulations to fine-grid simulations for a variety of oil recovery mechanisms in NFRs. This research provided the proper use of matrix-fracture transfer functions for different oil recovery mechanisms.

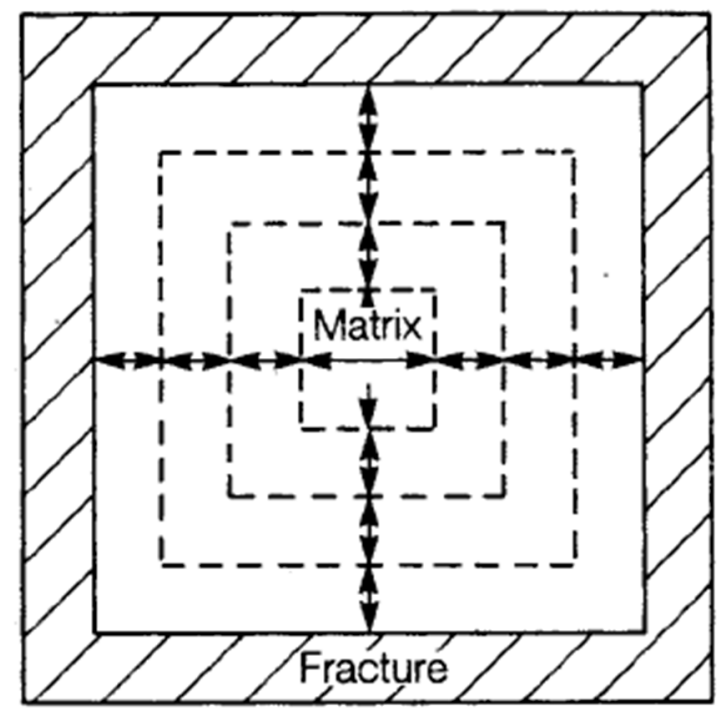

Figure 2.2: Discretization of a matrix block into a sequence of nested volume elements for the MINC method (from Wu and Pruess, 1988). 


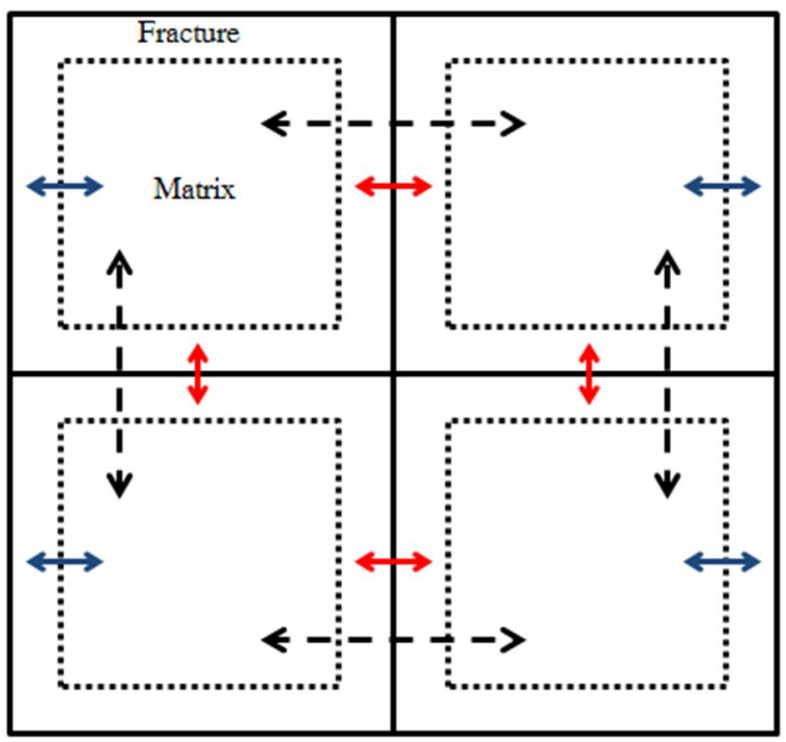

Fracture domain

Matrix domain

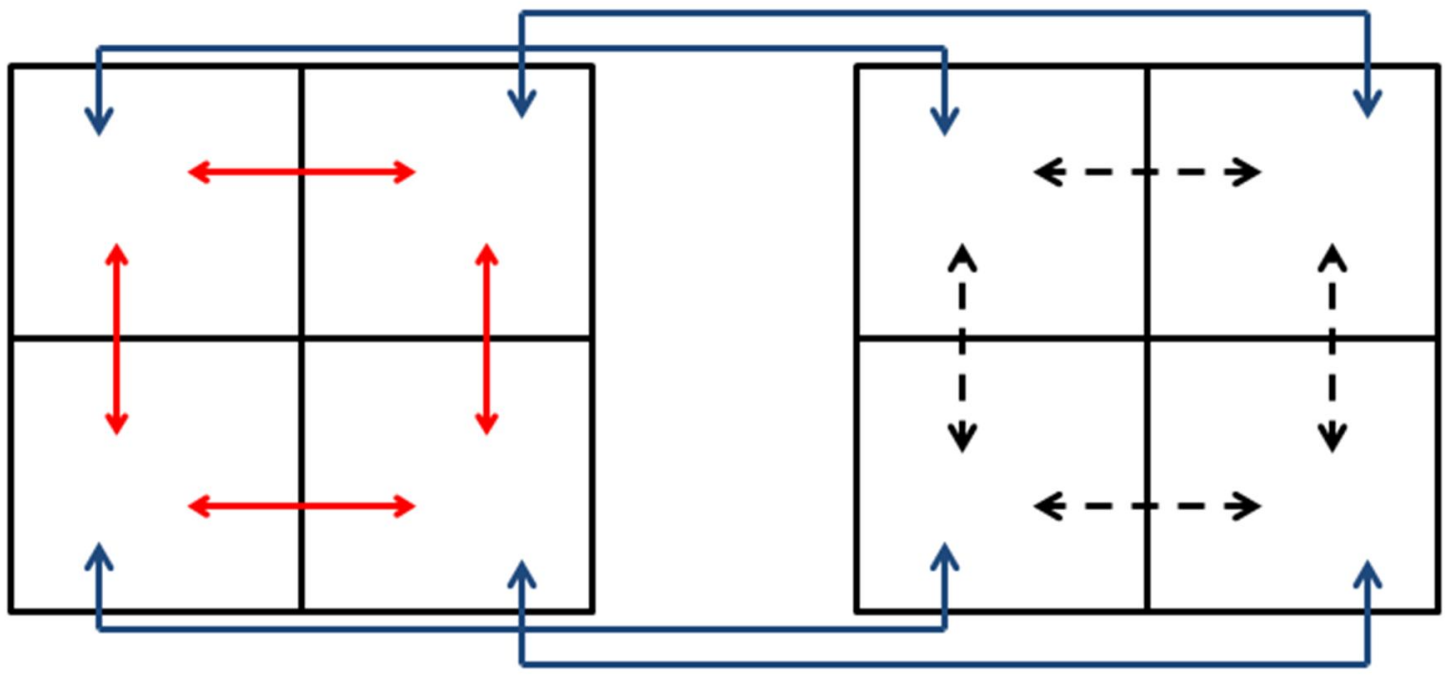

Figure 2.3: Schematic of connections in a dual permeability model. The matrix-tomatrix flow depicted by dashed double-headed arrows is neglected for dual porosity simulations. 


\subsubsection{Warren and Root Dual Porosity Solution}

The dual porosity approach for modeling NFRs was first introduced by Warren and Root (1963). They formulated the single-phase, one-dimensional flow from an infinite reservoir in a cylindrical coordinate system. In this section, we present detailed derivation of the analytical solution presented in Warren and Root (1963) and reproduce their results.

Equations 2.1, 2.2, and 2.3 can be combined and rewritten in a cylindrical coordinate system as follows for the fracture domain:

$$
\frac{k_{f}}{\mu}\left(\frac{1}{r} \frac{\partial}{\partial r}\left(r \frac{\partial P_{f}}{\partial r}\right)\right)-\frac{\sigma k_{m}}{\mu}\left(P_{m}-P_{f}\right)=\phi_{f} C_{f} \frac{\partial P_{f}}{\partial t}
$$

and for the matrix domain:

$$
\phi_{m} C_{m} \frac{\partial P_{m}}{\partial t}=\frac{\sigma k_{m}}{\mu}\left(P_{m}-P_{f}\right) .
$$

The initial conditions for fracture and matrix pressures are given by

$$
P_{f}(r, 0)=P_{m}(r, 0)=P_{i},
$$

where $P_{i}$ is the initial reservoir pressure. Likewise, the boundary conditions for the above partial differential equations can be expressed as

$$
\begin{aligned}
& P_{f}(\infty, t)=P_{m}(\infty, t)=P_{i}, \\
& q_{w}=\frac{k_{f}\left(2 \pi r_{w} h\right)}{\mu}\left(\frac{\partial P_{f}}{\partial r}\right)_{r=r_{w}},
\end{aligned}
$$

where $q_{w}, r_{w}$, and $h$ denote production rate, wellbore radius, and reservoir thickness, respectively. The equations for the fracture and matrix domains imply that at any given point fracture and matrix pressures co-exist. 
We define the following dimensionless parameters to simplify the mass conservation equation in conjunction with initial and boundary conditions:

$$
\begin{aligned}
& P_{D}=\left(\frac{2 \pi k_{f} h}{q \mu}\right)\left(P_{i}-P(r, t)\right), \\
& r_{D}=\frac{r}{r_{w}}, \\
& t_{D}=\left(\frac{k_{f}}{\left(C_{m} \phi_{m}+C_{f} \phi_{f}\right) \mu r_{w}^{2}}\right) t .
\end{aligned}
$$

Then, we calculate the partial derivatives in terms of the dimensionless variables using the chain rule, as given below:

$$
\begin{aligned}
& \frac{\partial P}{\partial t}=-\frac{q}{2 \pi h\left(C_{m} \phi_{m}+C_{f} \phi_{f}\right) r_{w}^{2}} \frac{\partial P_{D}}{\partial t_{D}}, \\
& \frac{\partial}{\partial r}=\frac{1}{r_{w}} \frac{\partial}{\partial r_{D}}, \\
& \frac{\partial P}{\partial r}=-\frac{q \mu}{2 \pi k_{f} h r_{w}} \frac{\partial P_{D}}{\partial r_{D}} .
\end{aligned}
$$

By substitution of Equations 2.17, 2.18, and 2.19 into Equations 2.9 and 2.10, the mass conservation equation for the fracture domain becomes:

$$
\begin{aligned}
& \frac{k_{f}}{\mu}\left(\frac{1}{r_{D} r_{w}} \frac{1}{r_{w}} \frac{\partial}{\partial r_{D}}\left(r_{D} r_{w}\left(-\frac{q \mu}{2 \pi k_{f} h r_{w}} \frac{\partial P_{f D}}{\partial r_{D}}\right)\right)\right) \\
& -\frac{\sigma k_{m}}{\mu}\left(-\frac{q \mu}{2 \pi k_{f} h}\left(P_{m D}-P_{f D}\right)\right) \\
& =\phi_{f} C_{f}\left(-\frac{q}{2 \pi h\left(C_{m} \phi_{m}+C_{f} \phi_{f}\right) r_{w}^{2}} \frac{\partial P_{f D}}{\partial t_{D}}\right),
\end{aligned}
$$


and for the matrix domain is given by the following:

$$
\begin{aligned}
& \phi_{m} C_{m}\left(-\frac{q}{2 \pi h\left(C_{m} \phi_{m}+C_{f} \phi_{f}\right) r_{w}^{2}} \frac{\partial P_{m D}}{\partial t_{D}}\right) \\
& =\frac{\sigma k_{m}}{\mu}\left(-\frac{q \mu}{2 \pi k_{f} h}\left(P_{m D-} P_{f D}\right)\right) .
\end{aligned}
$$

Also, the initial and boundary conditions in terms of dimensionless terms can be rewritten as

$$
\begin{aligned}
& P_{f D}\left(r_{D}, 0\right)=P_{m D}\left(r_{D}, 0\right)=0, \\
& P_{f D}\left(\infty, t_{D}\right)=P_{m D}\left(\infty, t_{D}\right)=0, \\
& \left(\frac{\partial P_{f D}}{\partial r_{D}}\right)_{r_{D}=1}=-1 .
\end{aligned}
$$

Two additional dimensionless parameters that result from the scaling are:

$$
\begin{aligned}
& \omega=\frac{C_{f} \phi_{f}}{C_{m} \phi_{m}+C_{f} \phi_{f}}, \\
& \lambda=\frac{\sigma k_{m} r_{w}^{2}}{k_{f}},
\end{aligned}
$$

where $\omega$ is a dimensionless parameter that represents the ratio of fluid capacitance or storage of the fracture to the total fluid capacitance of the combined system of matrix and fracture. The dimensionless parameter $\lambda$ govern matrix-fracture interporosity flow, which depends on the shape factor and the ratio of fracture to matrix permeability. Using dimensionless parameters $\omega$ and $\lambda$, the mass conservation equations (Equations 2.20 and 2.21) are simplified in the fracture domain to: 


$$
\frac{1}{r_{D}} \frac{\partial}{\partial r_{D}}\left(r_{D} \frac{\partial P_{f D}}{\partial r_{D}}\right)-\omega \frac{\partial P_{f D}}{\partial t_{D}}-(1-\omega) \frac{\partial P_{m D}}{\partial t_{D}}=0
$$

and in the matrix domain to:

$$
(1-\omega) \frac{\partial P_{m D}}{\partial t_{D}}+\lambda\left(P_{m D}-P_{f D}\right)=0 .
$$

To solve the above system of partial differential equations (PDE), the Laplace transformation is used to change the system of PDEs to a system of ordinary differential equations (ODE). Hence, the mass conservation equations can be rewritten in the Laplace domain for the fractures as

$$
\frac{1}{r_{D}} \frac{d}{d r_{D}}\left(r_{D} \frac{d P_{f D}}{d r_{D}}\right)-\omega\left(s P_{f D}\right)-(1-\omega)\left(s P_{m D}\right)=0,
$$

and for the matrix by

$$
(1-\omega)\left(s P_{m D}\right)+\lambda\left(P_{m D}-P_{f D}\right)=0 .
$$

The dimensionless pressure drop in the matrix $\left(P_{m D}\right)$ can be obtained from Equation 2.30 as

$$
P_{m D}=\frac{\lambda}{\lambda+(1-\omega) S} P_{f D} .
$$

By substitution of Equation 2.31 into Equation 2.29, the mass conservation equation for the fracture domain is simplified to:

$$
\begin{aligned}
& \frac{d^{2} P_{f D}}{d_{r_{D}}^{2}}+\frac{1}{r_{D}} \frac{d P_{f D}}{d r_{D}}-\omega s P_{f D}-(1-\omega) s \frac{\lambda}{\lambda+(1-\omega) s} P_{f D}=0, \\
& \frac{d^{2} P_{f D}}{d_{r_{D}}^{2}}+\frac{1}{r_{D}} \frac{d P_{f D}}{d r_{D}}-\left(\frac{s(\lambda+\omega(1-\omega) s)}{\lambda+(1-\omega) s}\right) P_{f D}=0 .
\end{aligned}
$$


Thus, the ordinary differential equation and the boundary conditions become:

$$
\begin{aligned}
& \frac{d^{2} P_{f D}}{d_{r_{D}}^{2}}+\frac{1}{r_{D}} \frac{d P_{f D}}{d r_{D}}-s f(s) P_{f D}=0, \\
& f(s)=\left(\frac{\lambda+\omega(1-\omega) s}{\lambda+(1-\omega) s}\right), \\
& P_{f D}\left(r_{D}=\infty\right)=0, \\
& \left(\frac{\partial P_{f D}}{\partial r_{D}}\right)_{r_{D}=1}=-\frac{1}{s} .
\end{aligned}
$$

The ODE of Equation 2.34 subject to the boundary conditions has the following analytical solution in the Laplace domain:

$$
P_{f D}\left(r_{D}\right)=\frac{K_{0}\left(r_{D} \sqrt{s f(s)}\right)}{s \sqrt{s f(s)} K_{1}(\sqrt{s f(s)})},
$$

where $K_{0}$ and $K_{l}$ are the modified Bessel functions of the second kind of order zero and one, respectively. Subsequently, the dimensionless pressure drop at the wellbore $\left(r_{D}=1\right)$ becomes

$$
P_{w D}=\frac{K_{0}(\sqrt{s f(s)})}{s \sqrt{s f(s)} K_{1}(\sqrt{s f(s)})} .
$$

Warren and Root (1963) presented an approximate solution in the time domain given by

$$
P_{w D}\left(t_{D}\right)=0.5\left\{\ln t_{D}+0.80908+E_{i}\left[\frac{-\lambda t_{D}}{\omega(1-\omega)}\right]-E_{i}\left[\frac{-\lambda t_{D}}{(1-\omega)}\right]\right\},
$$

where $E_{i}$ is the exponential integral. An alternative to this approximate solution is the Stehfest method, which can be used to calculate the inverse Laplace transform of 
Equation 2.39 (Stehfest, 1970). Figure 2.4 shows the dimensionless pressure drop versus the dimensionless time calculated by both methods for different values of $\lambda$ and $\omega$.

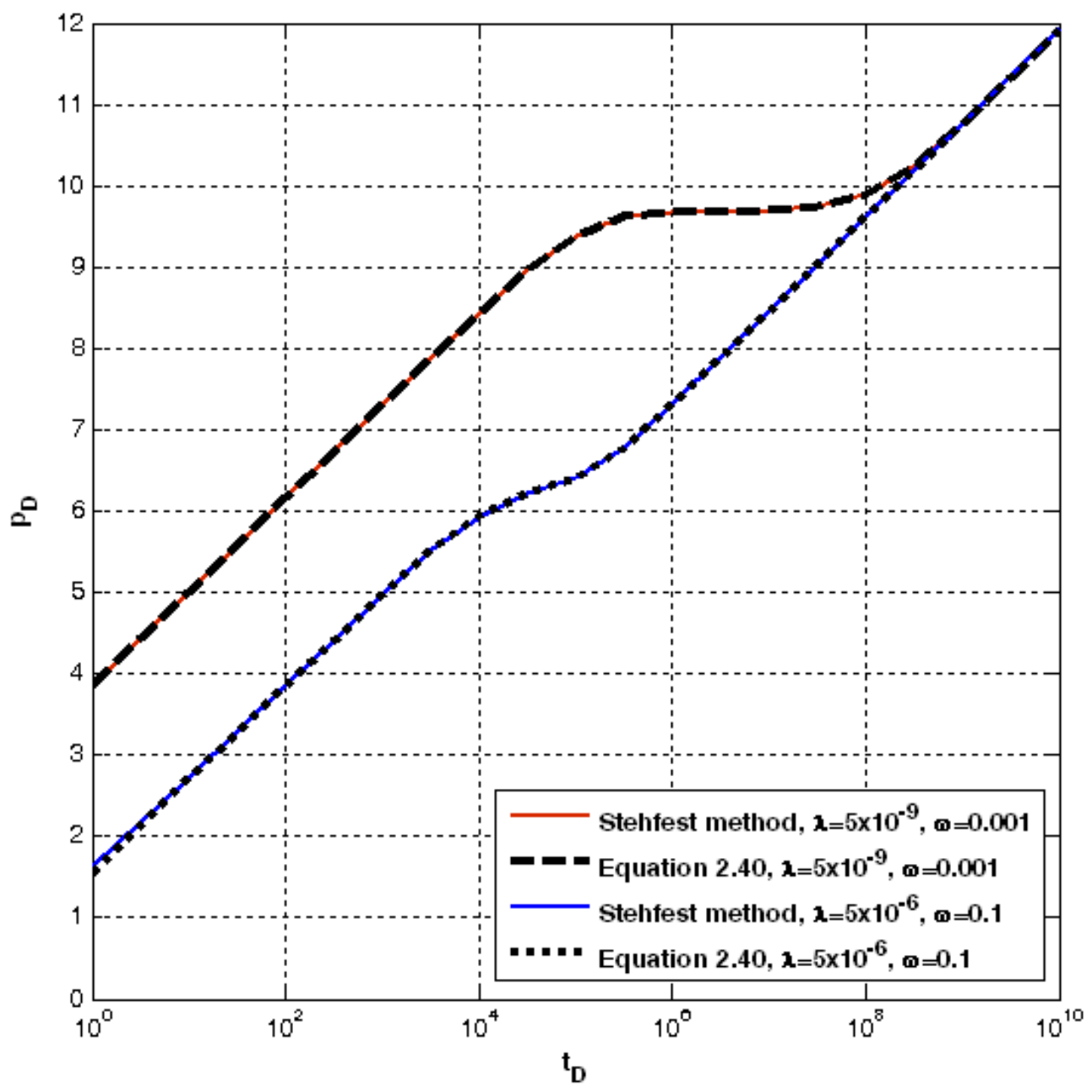

Figure 2.4: Dimensionless pressure response as a function of dimensionless time calculated by the approximate solution (Equation 2.40) and using the Stehfest method, corresponding to Figure 5 in Warren and Root (1963). 


\subsection{DisCRETE Fracture MODELS}

Outcrop characterization studies have shown that natural fractures vary substantially in height, length, and aperture, as well as spacing and network connectivity (Gillespie et al., 1993; Odling, 1997; Odling et al., 1999), thus highlighting a large discrepancy between reality and the uniformity inherent in dual porosity model assumptions. Hence, discrete fracture models (DFMs) were developed to reduce the number of non-physical abstractions inherent in dual continuum models. Most DFMs rely on unstructured grids to conform to the geometry and location of fracture networks. Compared to dual porosity models, DFMs offer several advantages. They account explicitly for the effect of individual fractures on fluid flow. Also, they are not overly constrained by grid-defined fracture geometries; hence the fracture model is easily adaptable and updatable. Moreover, the specification of the fluid exchange between matrix and fracture is more straightforward since it depends directly on the fracture geometry and any assigned relative permeability and capillary pressure functions. However, one disadvantage is that, in general, DFMs are numerically difficult to implement and computationally expensive. Further, one must be able to identify the locations and orientations of the discrete fractures for the model to be realistic.

Noorishad and Mehran (1982) presented a finite-element method for the transient solution of solute by dispersion and convection in 2D fractured porous media. Baca et al. (1984) proposed a 2D finite-element model for single-phase flow with heat and solute transport. Later, Juanes et al. (2002) presented a general finite-element model for 3D single-phase flow in fractured reservoirs. Kim and Deo (2000) and Karimi-Fard and Firoozabadi (2003) extended the finite-element method for two-phase fluid flow in fractured reservoirs including gravity and capillary effects. However, the DFMs based on finite-element procedures are not adequate for multiphase flow in highly heterogeneous 
reservoirs, since they do not ensure local mass conservation. Hoteit and Firoozabadi (2006) developed a compositional DFM using mixed finite-element and discontinuous Galerkin methods to resolve the mass conservation error for multiphase flows in NFRs.

Fu et al. (2005) developed a 3D, three-phase, black-oil, discrete fracture reservoir simulator based on the control-volume finite-element (CVFE) formulation. The CVFE method uses the same types of interpolation functions for dependent variables as those used in the finite element method. In contrast to the finite-element method in which the fluid potentials are approximated without the knowledge of fluxes between nodes, in the CVFE method fluid flux between nodes is calculated explicitly to ensure local mass conservation. Balasubramanian (2007) developed a compositional DFM using the control-volume finite-element method. Furthermore, Monteagudo and Firoozabadi (2004, 2007), Reichenberger et al. (2006), Matthai et al. (2007), Geiger et al. (2009), and Marcondes et al. (2010) applied control-volume finite-element methods to develop numerical simulators for multiphase flow in discrete fractured media.

Karimi-Fard et al. (2004) and Hui and Mallison (2009) developed DFMs based on an unstructured control-volume finite-difference formulation. This implementation is compatible with any reservoir simulator that represents grid connections by a connectivity list. The model employs the so-called lower dimensional approach to DFM gridding, where the rock matrix is modeled by $3 \mathrm{D}$ polyhedral cells and the fracture network is represented by a subset of the $2 \mathrm{D}$ interfaces separating grid cells. Figure 2.5 depicts a 2D example of fracture network defined in a physical domain. The physical domain is discretized using unstructured objects, representing the grid domain. For this $2 \mathrm{D}$ example, the matrix is represented by $2 \mathrm{D}$ control volumes and the fractures by $1 \mathrm{D}$ control volumes. As shown in Figure 2.5, each control volume is associated with a node. 
Although the fracture thickness is not represented in the grid domain, it is included in the computational domain for flow-rate evaluation.

To accurately capture the complexity of a fractured reservoir, it is usually necessary to use an unstructured discretization scheme. The above-mentioned DFM approaches require generating an unstructured grid to conform to the complexity of the fractures assigned to the domain of interest. Generation of such grid for an arbitrary fracture network can be a substantial challenge. Lee et al. (2000, 2001) and Li and Lee (2008) described a distinctly different DFM for simulating fluid flow in NFRs. Their model uses a conventional structured grid to represent the matrix and introduces additional fracture control volumes that are connected to the matrix through nonneighboring connections. Thus, the challenges associated with gridding fractures were circumvented entirely using Li and Lee's approach. Using non-neighboring connections to model fractures in a traditional finite-difference simulator is also discussed by Hearn et al. (1997) and Philip (2003).
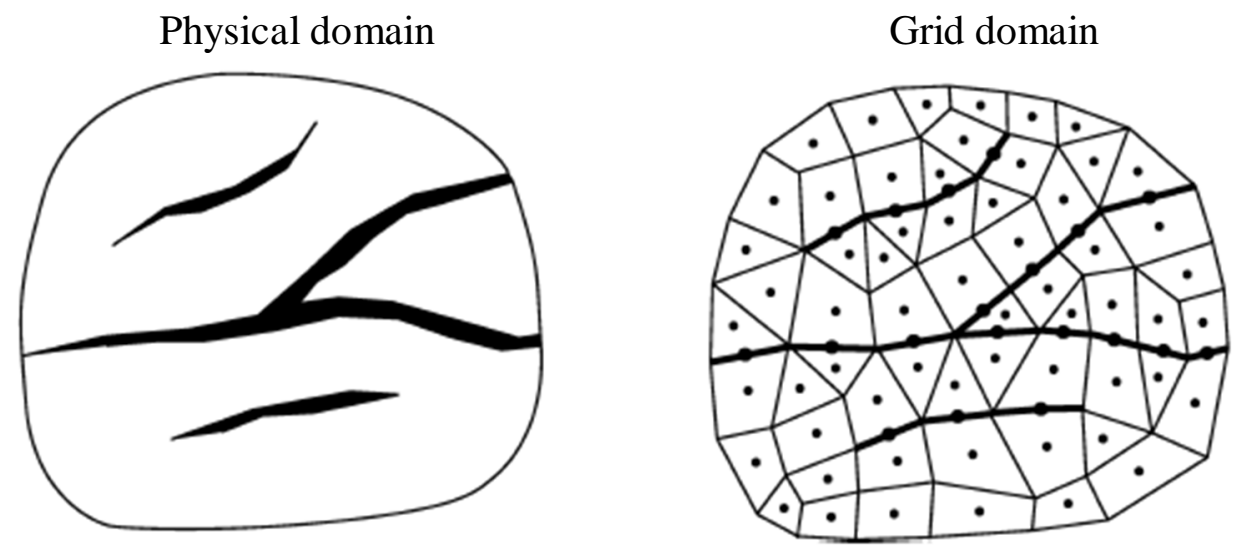

Figure 2.5: A 2D example of a fractured porous medium to be modeled using an unstructured control-volume finite-difference formulation. The thick line segments in the grid domain represent the fractures. One node is associated with each control volume (from Karimi-Fard et al., 2004). 


\subsection{AnAlytical Solutions For Hydraulically Fractured WellS}

Great effort has been made to model pressure transient behavior of hydraulically fractured vertical or horizontal wells. Many studies presented several analytical solutions for transient flow in fractured wells. Gringarten et al. (1974) developed transient flow solutions to predict the behavior of infinite-conductivity vertical fractures in homogenous formations. Cinco-Ley et al. (1978) and Cinco-Ley and Samaniego (1981) presented a semi-analytical model to analyze the pressure transient response of wells intercepted by a finite-conductivity vertical fracture. Rodriguez et al. (1984a and 1984b) derived semianalytical solutions for partially penetrating, infinite-conductivity and finite-conductivity fractures. Likewise, Tiab and Puthigai (1988) presented pressure-derivative type curves for a vertically fractured well located in an infinite reservoir.

Unlike vertical wells, horizontal wells are often hydraulically fractured at more than one point along the lateral section. Hegre and Larsen (1994) used the concept of effective wellbore radius to predict the performance of a horizontal well intercepted by multiple fractures. Also, Guo et al. (1994) developed methods for the performance prediction of horizontal wells with multiple fractures, but their work neglected the effect of interference between fractures. Later, Raghavan et al. (1997) developed a mathematical method based on finite-conductivity vertical-well-fracture models and improved the performance prediction of multiply-fractured horizontal wells. Moreover, Chen and Raghavn (1997) used the Ozkan and Raghavan's (1991) solution for a point source in a rectangular parallelepiped and derived expressions for the pressure transient behavior of a multiply-fractured horizontal well in a rectangular drainage region. Wan and Aziz (2002) described a new analytical solution for multiply-fractured horizontal wells where fractures partially penetrate the formation in the vertical direction. 


\subsection{LABORATORY EXPERIMENTS IN FRACTURED POROUS MEDIA}

A number of laboratory experiments have been conducted to investigate the behavior of various physical processes in fractured porous media. Mattax and Kyte (1962) performed water imbibition experiments in fractured cores and presented data that relate recovery behavior to matrix block size, fluid viscosity, and matrix permeability. Kyte (1970) conducted centrifuge tests on reservoir core samples to show that gravity segregation can sometimes be more important than capillary imbibition as a mechanism for oil recovery from matrix blocks. Kleppe and Morse (1974) and Kazemi and Merrill (1979) conducted water displacement experiments in fractured systems.

Horie et al. (1990) performed gas-oil gravity drainage experiments to investigate the importance of capillary continuity across a stack of matrix blocks. They showed that an important aspect of gas-oil gravity drainage in fractured reservoirs is the process of reinfiltration. That is, oil draining from an upper matrix block is not generally flowing through the fractures, but is rather entering a matrix block underneath. Barkve and Firoozabadi (1992) showed that oil reinfiltration is a function of both capillary and gravity forces. Firoozabadi and Markeset (1994) presented experimental data to demonstrate that the rate of oil drainage across a stack of matrix blocks is very sensitive to fracture aperture size. Dindoruk and Firoozabadi (1994) investigated flow through a liquid bridge between two matrix blocks and concluded that the fracture capillary pressure is the most critical parameter that affects the gas-oil gravity drainage process. They also pointed out that fracture liquid transmissibility has a small effect on gas-oil gravity drainage in fractured porous media. Firoozabadi et al. (1997) conducted several tests on viscous displacement in fractured cores, leading to the conclusion that viscous displacement can result in remarkable additional recovery in some fractured reservoirs beyond capillary-gravity equilibrium. Dindoruk and Firoozabadi (1997) developed an 
analytical 1D model based on the method of characteristics to study the effect of crossflow between matrix blocks and fractures on recovery.

Following the laboratory experiments conducted in fractured porous media, fracture models should be improved to incorporate more realistic physics into simulations. For instance, in order to include the effect of oil reinfiltration in gas-oil gravity drainage simulations, Por et al. (1989) developed a reservoir simulator based on dual porosity models with block-to-block interaction. They examined the effect of blockto-block interaction on oil recovery from a stack of matrix blocks and found that blockto-block interaction lowers the production rate and consequently delays oil recovery. Also, Fung (1991) and Uleberg and Kleppe (1996) studied gas-oil gravity drainage in dual porosity simulations by including the effect of oil reinfiltration. Likewise, Rubin (2007) developed a hybrid of subdomain and dual permeability models to simulate gasoil gravity drainage in the presence of reinfiltration and partial capillary continuity between matrix blocks. Wit et al. (2002) presented a procedure to calculate pseudo relative permeability curves for a stack of interacting blocks in gas-oil gravity drainage simulations.

Firoozabadi and Markeset (1994) carried out miscible displacement experiments in a number of matrix-fracture configurations. In their experiments, although the injected fluid had an early breakthrough, the injected solvent effluent concentration increased very slowly. This study revealed that miscible displacement in fractured porous media can be a very efficient process. Tan and Firoozabadi (1995) indicated that the solvent effluent concentration remains small in densely fractured systems even at high rates of injection. They concluded that fracture density has an important effect on miscible displacement efficiency. Beliveau et al. (1993) presented a $\mathrm{CO}_{2}$-flood pilot indicating that, contrary to conventional screening criteria, substantial tertiary oil recovery is attainable in a naturally 
fractured reservoir. Schechter and Guo (1998) conducted extensive experiments for $\mathrm{CO}_{2}$ gravity drainage in the naturally fractured Spraberry reservoir, leading to the conclusion that the matrix vertical permeability is a dominating factor affecting recovery efficiency during $\mathrm{CO}_{2}$ injection. Li et al. (2000) performed water imbibition followed by $\mathrm{CO}_{2}$ gravity drainage experiments on artificially fractured cores at reservoir conditions. The results of experiments showed that $\mathrm{CO}_{2}$ gravity drainage can substantially increase oil recovery after a water flood. Li et al. (2000) also found that the efficiency of $\mathrm{CO}_{2}$ gravity drainage decreases as the rock permeability decreases and the initial water saturation increases. Also, Darvish et al. (2006) experimentally showed that tertiary $\mathrm{CO}_{2}$ injection can be considered an effective enhanced oil recovery method in fractured reservoirs for targeting the residual oil after water injection. Trivedi and Babadagli (2006) experimentally investigated the efficiency of miscible displacement in fractured porous media and showed the dominance of phase diffusion into the matrix through fractures over viscous flow in the fracture. Moreover, Hoteit and Firoozabadi (2006) numerically studied diffusion in a fractured system for gas injection and concluded that diffusion can improve oil recovery in miscible displacements. In a recent study, Yanze and Clemens (2012) showed that non-equilibrium gas injection into a naturally fractured reservoir can improve oil production by gas-oil gravity drainage and diffusion of gas components from the fractures into the matrix. Based on their study, the effect of diffusion is pronounced when a large decrease in oil viscosity occurs as a result of gas components moving into the oil. 


\section{Chapter 3: General Purpose Adaptive Simulator (GPAS)}

The economic development of oil and gas reservoirs hinges in part on performing predictive simulations that enable reservoir engineers to select the best production strategies during all phases of field development. Reservoir simulation with geological and physical models has become an increasingly important tool for optimizing hydrocarbon recovery and reducing risk in development decisions. General Purpose Adaptive Simulator, GPAS, was developed at The University of Texas at Austin to provide an efficient and reliable environment to simulate a variety of oil recovery mechanisms. Moreover, the computational framework of GPAS allows for parallel processing, making it a good candidate for full-field simulations. The embedded discrete fracture model developed in this research for simulating fractured reservoirs is implemented into GPAS. Hence, we describe the framework, features, and different modules of GPAS in this chapter.

\subsection{OVERVIEW AND FRAMEWORK OF GPAS}

GPAS is a fully-implicit parallel-processing reservoir simulator comprising two main modules, the equation-of-state (EOS) compositional module and the chemical compositional module. Depending on the application, each module performs the related physical model calculations. The EOS compositional module of GPAS was first developed for miscible gas flooding simulations and the chemical compositional module of GPAS was later added to simulate polymer and surfactant enhanced oil recovery methods. The EOS compositional module solves phase behavior and mass balance 
equations for compositional gas flooding, while the chemical compositional module solves mass balance equations for aqueous phase components, which are present in polymer- and surfactant-flooding simulations.

The Integrated Parallel Accurate Reservoir Simulation (IPARS) was developed as a framework for parallel reservoir simulation research (Gropp et al., 1996; Parashar et al., 1997; Wheeler et al., 1999). The EOS and chemical compositional modules of GPAS are developed under this framework and thus, multi-processor simulations are feasible using GPAS. When using multiple processors, the simulation grid is evenly distributed among all processors. In order to maintain communication between different processors, a subgrid assigned to each processor is surrounded by a layer of grid elements that is shared with neighboring processors. This layer is also known as a ghost layer in parallel processing. It should be also noted that parallel processing calculations are separated from the physical model calculations.

The main features of the IPARS framework in conjunction with the compositional modules are memory allocation and management, domain decomposition, message passing between processors, and input/output processing. Here, we briefly describe these features:

- Memory Allocation and Management: The framework allocates memory for grid-element arrays. Such arrays store the grid-related properties such as porosity, permeability, and phase compositions. In order to implement a new physical model in a reservoir simulator under IPARS, we can define as many grid-element arrays as needed.

- Domain Decomposition: The reservoir domain is divided into several subdomains equal to the number of processors. Then, each subdomain is 
assigned to one processor that solves the physical model equations for that subdomain. The domain splitting in IPARS is along the y direction.

- Message Passing Between Processors: Communication (sending and receiving messages) between processors in the framework is done by message passing interface (MPI). The framework includes several routines that collect and distribute data between processors.

- Input/output Processing: The framework allows each processor to collect the input data for the portion of the reservoir that is assigned to it. Furthermore, at the output times, data from all processors are collected by a master processor. Hence, the framework includes several routines that read the input data from input files and write formatted data to output files.

In addition to the above-mentioned features, the framework performs several other calculations that are needed for various physical models. These calculations include:

- Calculation of the constant part of transmissibility between neighboring gridblocks

- Table look-up for relative permeability and capillary pressure calculations

- Identification of well locations with respect to grid and calculation of the corresponding well indices

- Identification of the ghost layers between processors

In the following sections, we describe the EOS and chemical compositional modules. However, since the embedded discrete fracture model (EDFM) is developed under the EOS compositional module, we present the formulation of this module in more details. 


\subsection{EOS COMPOSITIONAL Module}

Wang et al. (1997, 1999) developed a fully-implicit equation-of-state (EOS) compositional module in GPAS. This module employs the Peng-Robinson equation-ofstate (PR-EOS) for the hydrocarbon phase behavior calculations. Also, Darcy's law describes the multiphase flow in porous media. The number of stable hydrocarbon phases and their composition are determined using the EOS phase equilibrium calculations. Physical properties such as phase viscosity, density, and compressibility are calculated based on phase compositions. Hydrocarbon phases can possess many hydrocarbon components and mass transfer is allowed between oil and gas phases. However, there is no mass transfer between the aqueous and hydrocarbon phases. The aqueous phase contains water as a single component. Furthermore, the rock is slightly compressible and immobile.

All the developments in this research, which will be presented in the forthcoming chapters, are implemented in the EOS compositional module of GPAS. Hence, in the following sections, we describe the governing equations, phase behavior equations, and solution procedure of the EOS compositional module in GPAS.

\subsubsection{Governing Equations}

The number of hydrocarbon phases is denoted by $N_{p}$ and the aqueous phase consists of only the water component. Water is assumed to be slightly compressible with a constant viscosity. Each hydrocarbon phase is composed of $N_{c}$ hydrocarbon components. In the EOS compositional module of GPAS, multi-component and multiphase flow in a porous medium is entirely described using three types of equations when temperature is constant (isothermal reservoir). The governing equations of the EOS compositional model include: 
- $N_{c}$ material balance equations for hydrocarbon components,

- water material balance equation,

- $N_{c}$ phase equilibrium equations (equality of component fugacities), and

- pore volume constraint equation.

Hence, the total number of governing equations is $2 N_{c}+2$. Here, only two hydrocarbon phases are in equilibrium, resulting in $N_{c}$ phase equilibrium equations. Varavei (2009) developed an isothermal four-phase compositional model wherein three hydrocarbon phases (oil, gas, and second hydrocarbon liquid) are in equilibrium. The composition of the second hydrocarbon liquid phase is close to that of the gas phase, but its density is higher than the gas density. Four-phase physics can be relevant during $\mathrm{CO}_{2}$ injection at low temperatures. For the four-phase model, two sets of phase equilibrium equations $\left(2 N_{c}\right)$ are needed to describe mass transfer between phases, resulting in a total number of $3 N_{c}+2$ equations for each gridblock.

\subsubsection{Material Balance Equations}

In the EOS compositional module of GPAS, material balance equations for $N_{c}+1$ components (including water), in which Darcy's law represents phase transport from one gridblock to another, are given by

$$
V_{b} \frac{\partial}{\partial t}\left(\phi N_{i}\right)-V_{b} \nabla \cdot \sum_{j=1}^{N_{p}} \frac{\overline{\bar{k}} k_{r j}}{\mu_{j}} \xi_{j} x_{i j}\left(\nabla P_{j}-\gamma_{j} \nabla D\right)-q_{i}=0
$$

where $V_{b}, \phi, N_{i}, \overline{\bar{k}}, k_{r j}, \mu_{j}, \xi_{j}, x_{i j}, P_{j}, \gamma_{j}, D$, and $q_{i}$ denote bulk volume, porosity, moles of component $i$ per unit pore volume, absolute permeability tensor, relative permeability of phase $j$, viscosity of phase $j$, molar density of phase $j$, mole fraction of component $i$ in phase $j$, pressure of phase $j$, specific gravity of phase $j$, depth, and molar rate of 
component $i$ injected or produced, respectively. We note here that the EOS compositional module of GPAS does not include a dispersion term in the material balance equations. Haghshenas (2011) implemented a diffusion/dispersion model in the chemical compositional module of GPAS.

\subsubsection{Phase Equilibrium Equations}

Phase equilibrium equations for $N_{c}$ hydrocarbon components equate component fugacities between oil and gas phases. Phase equilibrium equation for component $i$ can be expressed as

$$
\begin{aligned}
& \ln \left(f_{i}^{g}\right)-\ln \left(f_{i}^{o}\right)=0 \\
& \ln K_{i}-\ln \varphi_{i}^{o}+\ln \varphi_{i}^{g}=0
\end{aligned}
$$

where $f_{i}^{j}, K_{i}$, and $\varphi_{i}^{j}$ represent the fugacity of component $i$ in phase $j$ (o: oleic, g: gaseous), equilibrium ratio of component $i$, and fugacity coefficient of component $i$ in phase $j$, respectively. For the four-phase model in which three hydrocarbon phases are in equilibrium, another set of equations equate component fugacities between oil and the second liquid phases.

\subsubsection{Pore Volume Constraint Equation}

The volumetric constraint equation, also known as the phase saturation constraint, implies that the pore volume in each cell must be filled completely by the total fluid volume, and is given by 


$$
\begin{aligned}
& \sum_{j=1}^{N_{p}} \frac{N_{j}}{\xi_{j}}=1, \\
& \sum_{i=1}^{N_{c}+1} N_{i} \sum_{j=1}^{N_{p}} L_{j} \bar{v}_{j}-1=0,
\end{aligned}
$$

where $N_{i}, N_{j}, L_{j}, \bar{v}_{j}$, and $\xi_{j}$ denote moles of component $i$ per pore volume, moles of phase $j$ per pore volume, mole fraction of phase $j$, molar volume of phase $j$, and molar density of phase $j$, respectively.

\subsubsection{Phase Behavior Calculations}

At each time-step in an EOS compositional simulation, the number of hydrocarbon phases, phase mole ratios, and phase compositions are calculated. The algorithm used in GPAS for phase equilibrium calculations was developed by Perschke et al. (1989). The procedure of this algorithm is a sequential application of stability test and flash calculation. From the overall mole fraction, the stability test determines the number of phases that can exist at the prevailing condition. There are two methods for the stability test in GPAS: the stationary point location method (Michelsen, 1982) and Gibbs free energy minimization method (Trangenstein, 1987). Likewise, flash calculation computes the phase mole ratios and phase compositions. There are two methods for the flash calculation in GPAS: the accelerated successive substitution (ACSS) method (Mehra et al., 1983) and Gibbs free energy minimization (Trangenstein, 1987). The phase fugacity equations are then solved along with other governing equations to update primary variables. 
In order to perform phase equilibrium calculations, various equation-of-states (EOS) are used in different reservoir simulators. As previously mentioned, the PengRobinson (Peng and Robinson, 1976) equation-of-state (PR-EOS) is used in the GPAS compositional model, which is expressed as

$$
P=\frac{R T}{v-b}-\frac{a(T)}{v(v+b)+b(v-b)},
$$

where $P, v, T$, and $R$ are the pressure, volume, temperature, and gas constant, respectively. Also, the parameters $a(T)$ and $b$ for a pure component are calculated from

$$
\begin{aligned}
& a(T)=0.45724 \frac{\alpha\left(R T_{c}\right)^{2}}{P_{c}}, \\
& b=0.0778 \frac{R T_{c}}{P_{c}}, \\
& \alpha=\left[1+m\left(1-\sqrt{\frac{T}{T_{c}}}\right)\right]^{2} \\
& m=\left\{\begin{array}{c}
0.37464+1.54226 \omega-0.26992 \omega^{2} \quad(\text { if } \omega<0.49) \\
0.37964+1.48503 \omega-0.164423 \omega^{2}+0.016666 \omega^{3} \quad(\text { if } \omega>0.49)
\end{array}\right.
\end{aligned}
$$

where $P_{c}, T_{c}$, and $\omega$ are the critical pressure, critical temperature, and acentric factor, respectively. In order to use PR-EOS for multi-component mixtures, $a$ and $b$ for phase $j$ should be computed from the mixing rules:

$$
\begin{aligned}
a_{m, j} & =\sum_{i=1}^{N_{c}} \sum_{k=1}^{N_{c}} x_{i j} x_{k j} \sqrt{a_{i} a_{k}}\left(1-\delta_{i k}\right), \\
b_{m, j} & =\sum_{j=1}^{N_{C}} x_{i j} b_{i}
\end{aligned}
$$


where $a_{i}$ and $b_{i}$ are computed for each component from Equations 3.7 and 3.8 and $x_{i j}$ is the mole fraction of component $i$ in phase $j$. Likewise, $\delta_{i k}$ is called the binary interaction coefficient between components $i$ and $k$. The Peng-Robinson EOS for phase $j$ can be rewritten in terms of the phase compressibility factor $(Z)$ as follows:

$$
\begin{aligned}
& Z_{j}^{3}+\left(B_{j}-1\right) Z_{j}^{2}+\left(A_{j}-3 B_{j}^{2}-2 B_{j}\right) Z_{j}+\left(-A_{j} B_{j}+B_{j}^{2}+B_{j}^{3}\right)=0, \\
& Z_{j}=\frac{P v_{j}}{R T}, \\
& A_{j}=\frac{a_{m, j} P}{(R T)^{2}}, \\
& B_{j}=\frac{b_{m, j} P}{R T} .
\end{aligned}
$$

Using the PR-EOS, the fugacity coefficient of component $i$ in phase $j$ (which is needed in Equation 3.3) is calculated by

$$
\begin{aligned}
\ln \varphi_{i}^{j}=\frac{b_{i}}{b}\left(Z_{j}-1\right)-\ln \left(Z_{j}-B_{j}\right) & \\
& -\frac{A_{j}}{2 \sqrt{2} B_{j}}\left(\frac{2}{a_{j}} \sum_{m=1}^{N_{c}} x_{m j} a_{i m}-\frac{b_{i}}{b_{m}}\right) \ln \left(\frac{Z_{j}+(1+\sqrt{2}) B_{j}}{Z_{j}+(1-\sqrt{2}) B_{j}}\right) .
\end{aligned}
$$

Also, the equilibrium ratio of component $i$ (also known as the $K$-value) is the ratio of component mole fraction in the gaseous phase to the component mole fraction in the oleic phase. 


\subsubsection{Primary Variables and Solution Procedure}

The $2 N_{c}+2$ primary (independent) variables used in the EOS compositional module of GPAS are $P_{w}, N_{1}, \ldots, N_{n c}, N_{w}, \ln K_{1}, \ldots, \ln K_{n c}$. Therefore, the number of nonlinear equations and unknowns for a reservoir represented by $N B$ gridblocks is $\left(2 N_{c}+2\right) \times N B$. The partial differential equations for the component mass balances are discretized on a Cartesian grid using the one-point upstream weighting scheme for the transmissibility terms. The discretized component mass balance equations along with the phase equilibrium equations and pore volume constraint equation are then linearized in terms of the primary variables.

The Newton's method is used to linearize the non-linear system of equations as given below:

$$
J \Delta X=-R,
$$

where, $R$ is the residual vector for all governing equations of all gridblocks. Also, vector $X$ contains all unknowns (primary variables) for all gridblocks. Furthermore, $J$ is the Jacobian matrix that includes the derivatives of all equations (residuals) with respect to all primary variables. Therefore, Equation 3.18 can be rewritten as

$$
\left(\begin{array}{cccc}
J_{1,1} & J_{1,2} & \cdots & J_{1, N B} \\
J_{2,1} & J_{2,2} & \cdots & J_{2, N B} \\
\vdots & \vdots & & \vdots \\
J_{N B, 1} & J_{N B, 2} & \cdots & J_{N B, N B}
\end{array}\right)\left(\begin{array}{c}
\Delta X_{1} \\
\Delta X_{2} \\
\vdots \\
\Delta X_{N B}
\end{array}\right)=-\left(\begin{array}{c}
R_{1} \\
R_{2} \\
\vdots \\
R_{N B}
\end{array}\right),
$$

where $J_{i, j}$ represents the derivatives of the governing equations (residuals) of the gridblock $i$ with respect to the primary variables in gridblock $j$. That is, 


$$
\begin{aligned}
& \left(\begin{array}{cccccccc}
\frac{\partial R_{1, i}^{f}}{\partial \ln K_{1, j}} & \cdots & \frac{\partial R_{1, i}^{f}}{\partial \ln K_{n_{c}, j}} & \frac{\partial R_{1, i}^{f}}{\partial N_{1, j}} & \cdots & \frac{\partial R_{1, i}^{f}}{\partial N_{n_{c}, j}} & \frac{\partial R_{1, i}^{f}}{\partial P_{j}} & \frac{\partial R_{1, i}^{f}}{\partial N_{w, j}}
\end{array}\right.
\end{aligned}
$$

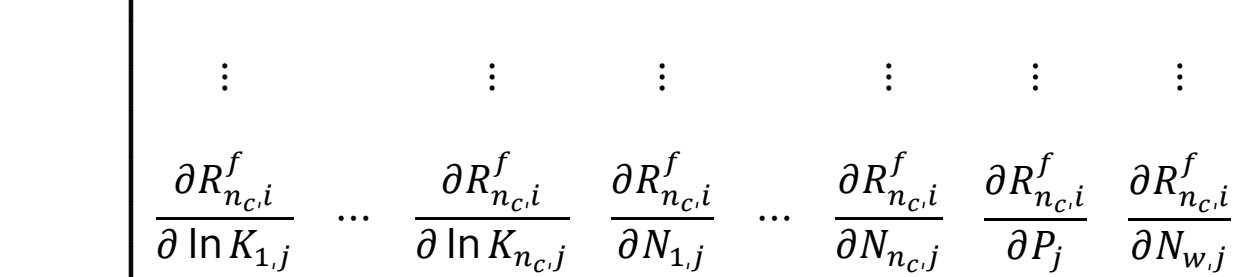

$$
\begin{aligned}
& J_{i, j}=\mid \begin{array}{llllllll}
\frac{\partial R_{i}^{v}}{\partial \ln K_{1, j}} & \cdots & \frac{\partial R_{i}^{v}}{\partial \ln K_{n_{c}, j}} & \frac{\partial R_{i}^{v}}{\partial N_{1, j}} & \cdots & \frac{\partial R_{i}^{v}}{\partial N_{n_{c}, j}} & \frac{\partial R_{i}^{v}}{\partial P_{j}} & \frac{\partial R_{i}^{v}}{\partial N_{w, j}}
\end{array} \\
& \frac{\partial R_{1, i}^{m}}{\partial \ln K_{1, j}} \quad \cdots \quad \frac{\partial R_{1, i}^{m}}{\partial \ln K_{n_{c}, j}} \quad \frac{\partial R_{1, i}^{m}}{\partial N_{1, j}} \quad \cdots \quad \frac{\partial R_{1, i}^{m}}{\partial N_{n_{c}, j}} \quad \frac{\partial R_{1, i}^{m}}{\partial P_{j}} \quad \frac{\partial R_{1, i}^{m}}{\partial N_{w, j}}
\end{aligned}
$$

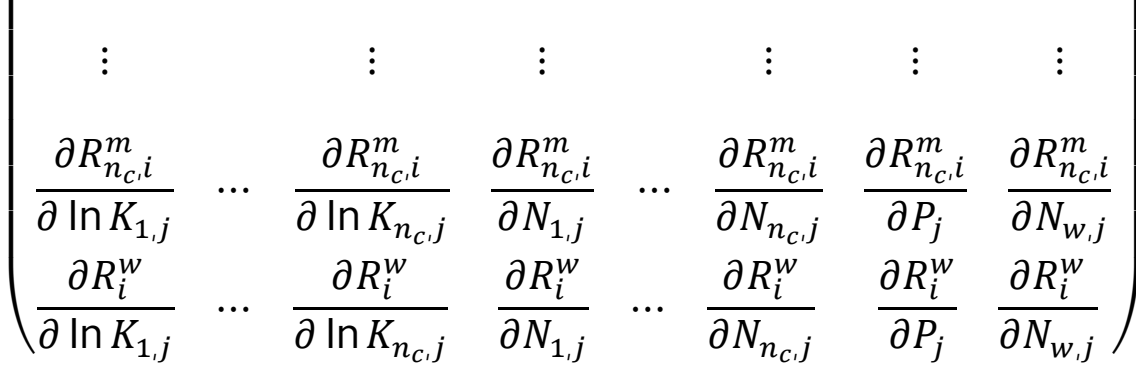

$$
\begin{aligned}
& \Delta X_{j}=\left(\Delta \ln K_{1}, \ldots, \Delta \ln K_{n_{c}}, \Delta N_{1}, \ldots, \Delta N_{n_{c}}, \Delta P, \Delta N_{w}\right)_{j}, \\
& R_{i}=\left(R_{1}^{f}, \ldots, R_{n_{c}}^{f}, R^{v}, R_{1}^{m}, \ldots, R_{n_{c}}^{m}, R^{w}\right)_{i}
\end{aligned}
$$

The superscripts $f, v, m$, and $w$ refer to fugacity (phase equilibrium) equations, volume constraint equation, mass balance equations for hydrocarbon components, and water mass balance equation, respectively.

The linear system is then solved for the independent variables of all gridblocks. The linear solvers from the PETSc package (Portable Extensible Toolkit for Scientific Computation) are used to solve the underlying linear system of equations (Balay et al., 1998). Once $\Delta X$ is calculated from Equation 3.18, the vector of unknowns $(X)$ can be updated using the following equation: 


$$
X^{\text {new }}=X^{\text {old }}+\Delta X
$$

Applying the Newton's method, the above procedure is repeated until the convergence criteria based on the residual tolerances are met.

\subsection{Chemical Compositional Module}

In the EOS compositional module of GPAS, the aqueous phase contains water as a single component and therefore, this module cannot model chemical enhanced oil recovery (EOR) processes. In order to perform chemical EOR simulations, the chemical module of GPAS was first developed by Nalla (2002), who implemented a chemical model for conservative tracers and polymers. John et al. (2005) extended Nalla's work to model partitioning tracer, chemical species adsorption, and surfactant phase behavior. In these models, the material balance equations for hydrocarbon were solved implicitly, while the material balance equations for the aqueous phase components were solved explicitly after updating the saturations, densities, and phase fluxes.

Later, Han et al. (2007) and Fathi-Najafabadi et al. (2009) developed fullyimplicit chemical models in GPAS. In the new formulation, the material balance equations for both aqueous phase components and hydrocarbon components were solved implicitly. The governing equations in the chemical module are material balance equations and the volume constraint equation. Three phases (water, oil, and microemulsion) can be present in the simulations depending on the composition and the phase behavior of water/oil/surfactant. The microemulsion phase is defined as a thermodynamically stable phase that contains oil, water, and surfactant. 


\subsection{Parallel Processing}

Large-scale simulation of petroleum reservoirs with complex recovery processes is challenging owing to the problem size and extensive calculations involved. Parallel processing with high performance computing clusters can alleviate these challenges to a good extent. Wang et al. (1997), Zhang et al. (2001), Dogru et al. (2002), Gai et al. (2003), and DeBaun et al. (2005) demonstrated the necessity, advantages, and applicability of using parallel processing for large-scale reservoir simulations.

In order to perform parallel reservoir simulation, the reservoir domain is divided into several subdomains equal to the number of processors. Each processor collects the properties of the cells that belong to it from input files. Likewise, at the output times, a master processor collects the data from all processors. As previously described, IPARS provides the parallel processing framework for GPAS. Under this framework, the reservoir domain is divided in the $\mathrm{y}$ direction into several subdomains equal to the number of processors.

When using parallel processors, each processor should communicate with other processors at every time-step. Hence, a surrounding layer of cells (ghost layer) is added to each subdomain as a communication layer. Communication between ghost layers is established by a message passing interface (MPI) in the framework. The ideal speed-up for a parallel reservoir simulation with $n$ processors is equal to $n$. However, in reality, the speed-up is less than $n$ owing to the communications between processors and memory contention. In general, as the number of processors increases, the communication cost between processors increases. 


\section{Chapter 4: Embedded Discrete Fracture Model (EDFM)}

Accurate modeling and simulation of naturally fractured reservoirs is still challenging owing to permeability anisotropies and contrasts. Non-physical abstractions inherent in conventional dual porosity and dual permeability models make them inadequate for solving different fluid-flow problems in fractured reservoirs. Also, recent technologies for discrete fracture modeling may suffer from large computational times and the industry has not used such approaches widely, even though they give more accurate representations of fractured reservoirs than dual continuum models.

In this chapter, we describe a methodology for modeling vertical and slanted fractures using an embedded discrete fracture model (EDFM), which is developed for the EOS compositional module of GPAS. In contrast to dual continuum models, fractures have arbitrary orientations and can be oblique or vertical, honoring the complexity of a typical naturally fractured reservoir. Furthermore, unlike other discrete fracture models, the EDFM approach uses a structured grid for the simulation of complex fracture networks. This approach is based on non-neighboring connections that will be explained in this chapter. The implementation of EDFM includes two parts:

- Pre-processing of a fracture network with any geometry over an arbitrary grid to provide the required data for reservoir simulation.

- Implementation of non-neighboring connections, transmissibility modifiers, and other necessary changes into the reservoir simulator, which is GPAS in this research. 


\subsection{OVERVIEW OF EDFM}

As described in Chapter 2, discrete fracture models (DFMs) are a new class of models for simulating fluid flow in NFRs and provide more realistic representation of NFRs than conventional dual porosity and dual permeability models. However, to the best of our knowledge, DFMs have not been widely used in the industry for field-scale reservoir simulation studies. In general, when using DFMs, a large number of small cells must be used near the fractures, which may result in increased computational times. Also, most DFMs require generating an unstructured grid to conform to the complexity of the fractures presented in the domain of interest. Generation of such grid for an arbitrary fracture network can be a substantial challenge and creates more complexity for real field simulations.

Considering the possible disadvantages of DFMs, Li and Lee (2008) proposed a discrete fracture model for simulating flow in NFRs, called the embedded discrete fracture model (EDFM). The model uses a structured grid to represent the matrix, and introduces additional fracture control volumes by computing the intersection of fractures with the matrix grid. Therefore, challenges associated with unstructured gridding are bypassed entirely. Moreover, this approach can be easily incorporated in existing finite difference reservoir simulators.

Li and Lee (2008) extended the approach described by Lee et al. $(2000,2001)$, who modeled large-scale fractures explicitly as a two-dimensional plane crossing multiple cells and presented a systematic way to calculate transport parameters between fractures and the discretized homogenized matrix medium. Lee et al. $(2000,2001)$ formulated the fluid transport between a matrix cell and a segment of a fracture embedded in that cell as 


$$
q_{m f}=T_{m f}\left(\psi_{m}-\psi_{f}\right),
$$

where $q_{m f}$ is the volumetric rate between the matrix gridblock and the fracture segment, $T_{m f}$ is the transmissibility between them, $\psi_{m}$ is the matrix gridblock potential, and $\psi_{f}$ is the fracture potential. They applied the similar concept of wellbore productivity index introduced by Peaceman $(1978,1983)$ and Lee and Milliken (1993) to derive the transport index between matrix and fractures in a grid cell. In doing so, they formulated fluid flow as a well-like equation inside the fracture and a source/sink term between fracture and matrix. The source/sink term allows for coupling multiphase flow equations in fractures and matrix. The pressure is assumed to vary linearly in the normal direction to each fracture. Furthermore, flow in the fracture blocks is governed by Darcy's equation, the same as flow in the matrix medium. For simplicity of geometric design, Lee et al. (2000, 2001) and Li and Lee (2008) implemented the EDFM approach only for vertical fractures. That is, fractures are approximated by vertical planar rectangles, but have arbitrary orientations in the horizontal plane.

In the studies conducted by Lee et al. (2001) and Li and Lee (2008), the EDFM approach models long fractures in a hierarchical fracture modeling framework. Most NFRs comprise numerous small-scale fractures (micro-fractures) and sporadic large-scale fractures (macro-fractures). Micro-fractures are typically shorter than computational grid dimensions while macro-fractures are field-scale features extending through multiple gridblocks. Considered from the standpoint of flow behavior, macro-fractures have a first order effect on fluid flow whereas micro-fractures are less important. In the hierarchical fracture modeling framework, micro-fractures are homogenized by changing effective properties of matrix gridblocks and large-scale fractures are explicitly modeled by a discrete fracture model (Clemo and Smith, 1997; Lee et al., 2001). In order to 
homogenize small-scale fractures, Oda (1985) derived a simple analytical expression for enhanced matrix permeability for gridblocks that contain very short fractures. Likewise, Lough et al. $(1997,1998)$ developed a boundary element method (with uniform pressure and periodic boundary conditions) to calculate effective conductivity for gridblocks containing multiple fractures.

We have implemented the EDFM approach presented by Li and Lee (2008) in GPAS. More importantly, we have extended this method for modeling inclined fractures. The developed model allows for multiple sets of fractures with any dip and strike angle. Field characterization studies have shown that the occurrence of obliquely dipping fractures is quite common in naturally fractured reservoirs (Walsh and Watterson, 1988; Angerer et al., 2002; Grechka and Tsuankin, 2004). In the next sections, we describe the methodology used for implementing the EDFM approach in GPAS.

\subsection{Methodology}

The EDFM approach borrows the dual-medium concept from conventional dual continuum models, but also incorporates the effect of each fracture explicitly. In this approach, computational fracture control volumes are not present in the vicinity of matrix gridblocks, but are defined in a separate computational domain. In general, the same gridblock sizes are used for both matrix and fracture domains; however, there is no constraint for both gridblock sizes to be the same in this approach. In order to define fracture control volumes in the fracture domain, the intersection of all fractures with the matrix grid should be computed first. 


\subsubsection{Fracture Discretization}

Vertical and inclined fractures are discretized vertically and horizontally by the cell boundaries of the matrix grid. The intersection of a vertical fracture and a matrix gridblock is always a rectangle, as shown in Figure 4.1. However, when an arbitrarilyoriented fracture plane passes through a gridblock, regular intersections are polygons with $3,4,5$, or 6 corners. Figure 4.2 shows possible intersections of an inclined fracture plane and a matrix gridblock, which can be a triangle, quadrilateral, pentagon, or hexagon. An exact specification of these intersections is important for calculating the connection between matrix and fracture.

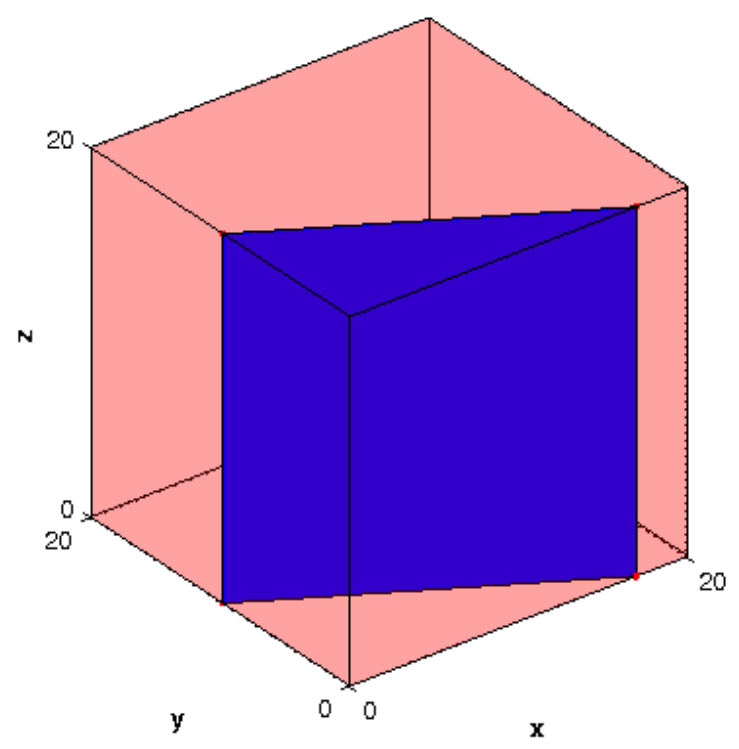

Figure 4.1: Intersection of a vertical fracture plane and a matrix gridblock, which is always a rectangle. 

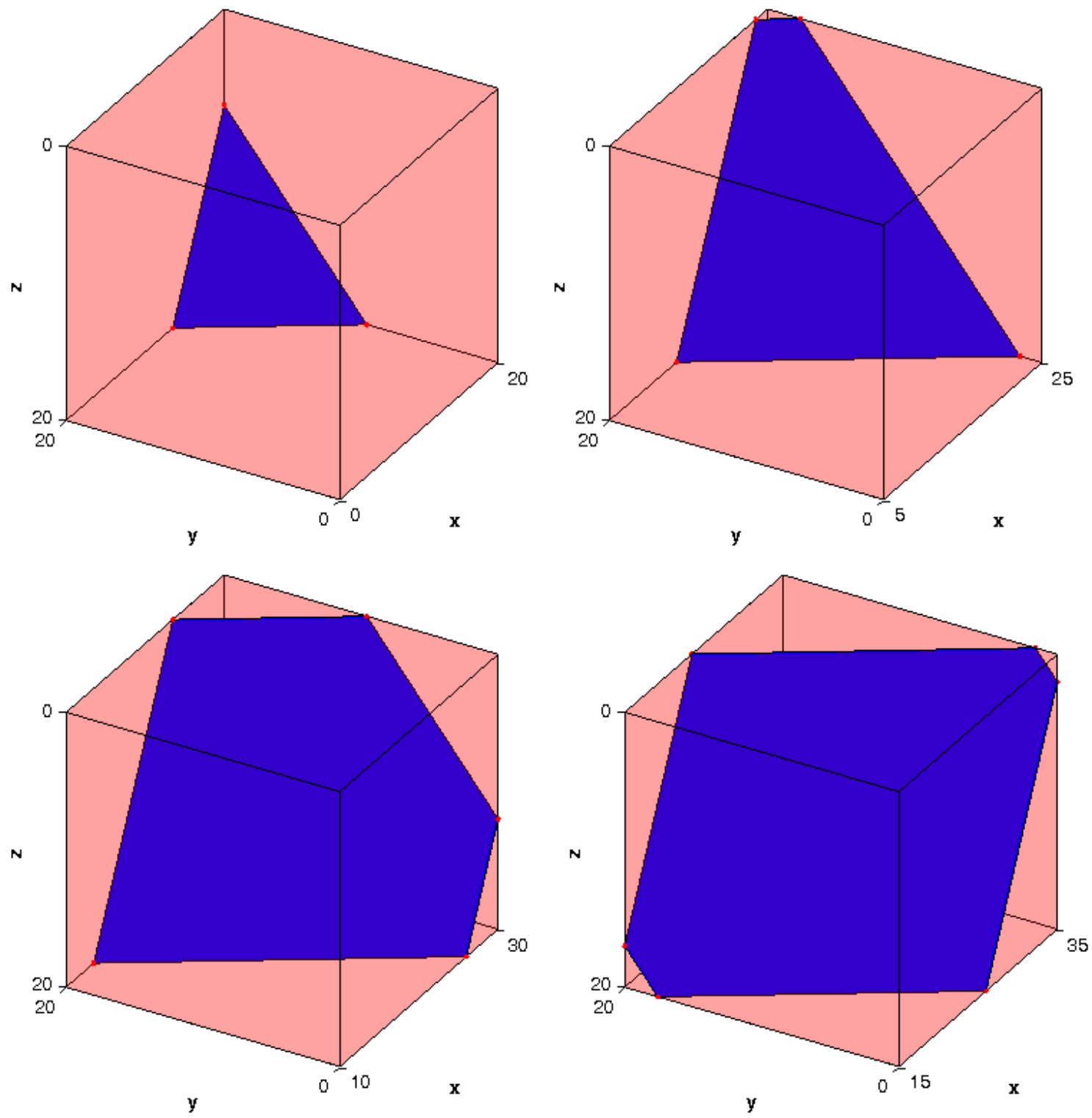

Figure 4.2: Possible intersections of an inclined fracture plane and a matrix gridblock, which can be a triangle, quadrilateral, pentagon, or hexagon. 
A fracture cell should be defined in the fracture domain corresponding to each gridblock containing a segment of a fracture plane. Figure 4.3 shows a simple two-layer model comprising two tilted fractures, inclined 60 and 75 degrees from the horizontal plane. Both fractures penetrate the entire height of the reservoir. The structured grid for the matrix is $10 \times 10 \times 2$ cells in the $\mathrm{x}, \mathrm{y}$, and $\mathrm{z}$ directions, respectively, with cell dimensions of $20 \times 20 \times 20$ feet in all directions.

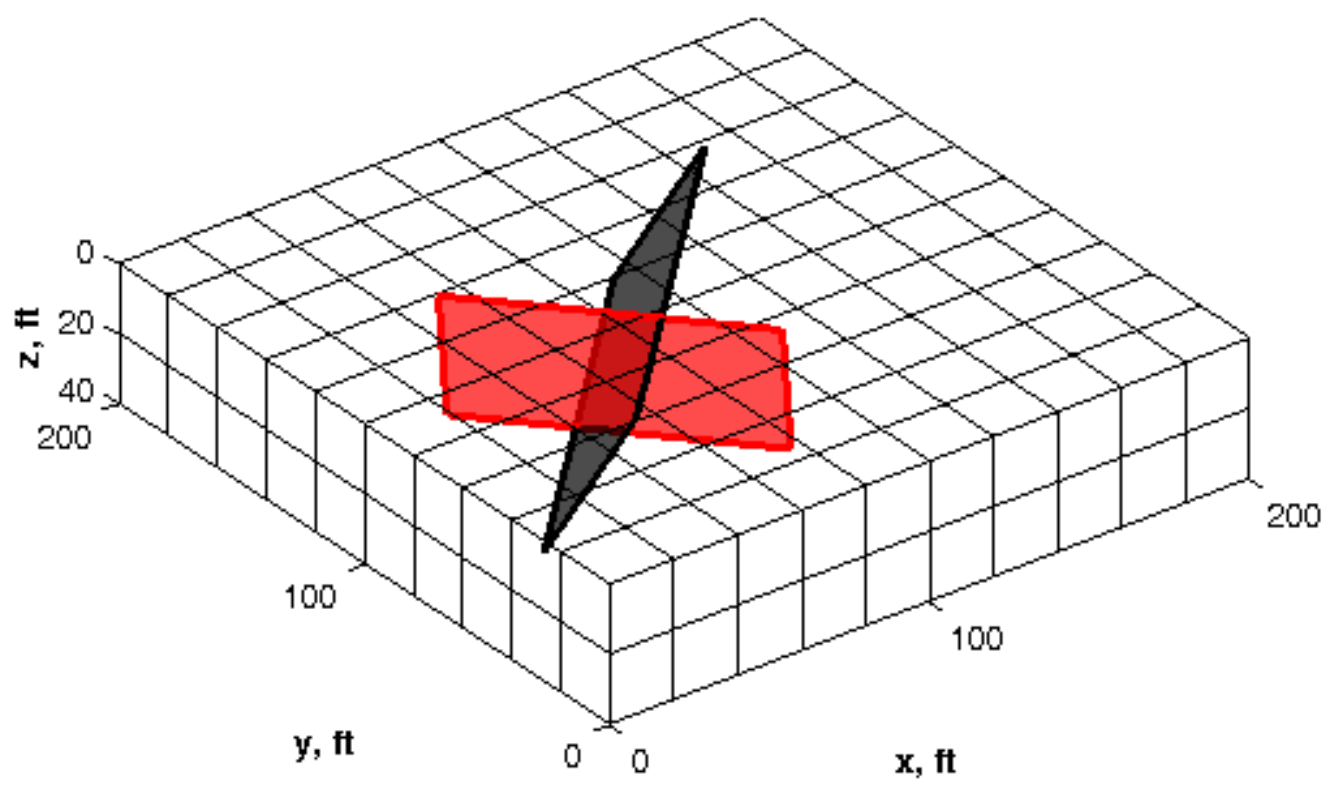

Figure 4.3: Geometrical representation of a fractured model $(200 \mathrm{ft} \times 200 \mathrm{ft} \times 40 \mathrm{ft})$ used to illustrate the methodology applied in EDFM. The model comprises two tilted fractures, black and red fractures, which are inclined 60 and 75 degrees from the horizontal plane, respectively. The matrix grid is $10 \times 10 \times 2$ cells in the $\mathrm{x}, \mathrm{y}$, and $\mathrm{z}$ directions, respectively, with cell dimensions of $20 \times 20 \times 20 \mathrm{ft}$ in all directions. 
Figure 4.4 specifies all gridblocks containing a segment of a fracture for both layers. Cells containing a segment of the black fracture are marked with black circles and those containing a segment of the red fracture are marked with red squares. In contrast to vertical fractures that cross similar gridblocks in different computational layers, inclined fractures may cross a different number of gridblocks in each layer, as shown in Figure 4.4. For the example under consideration, the black fracture penetrates 14 cells in the top computational layer while penetrating only 9 cells in the bottom one. Unlike the black fracture, the red fracture crosses more gridblocks in the bottom computational layer than in the top one.

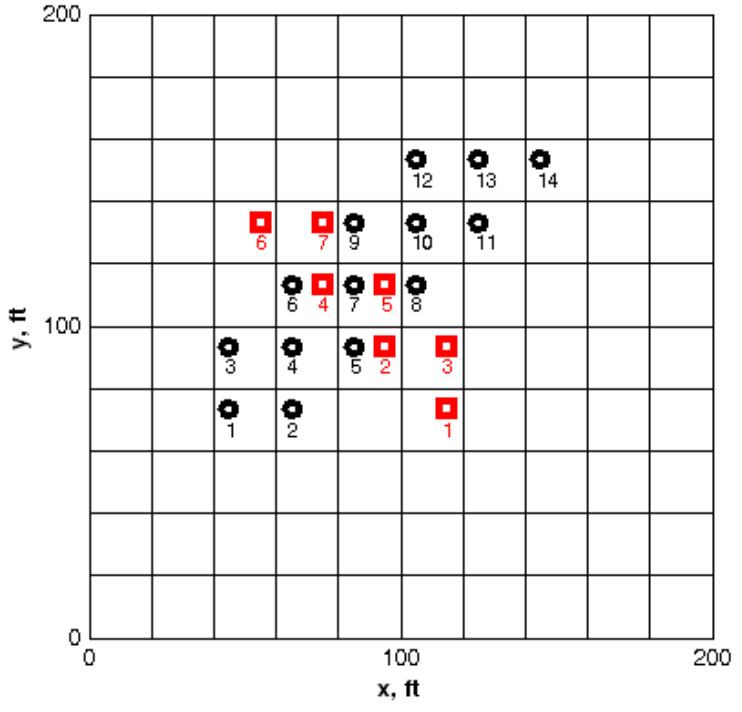

(a)

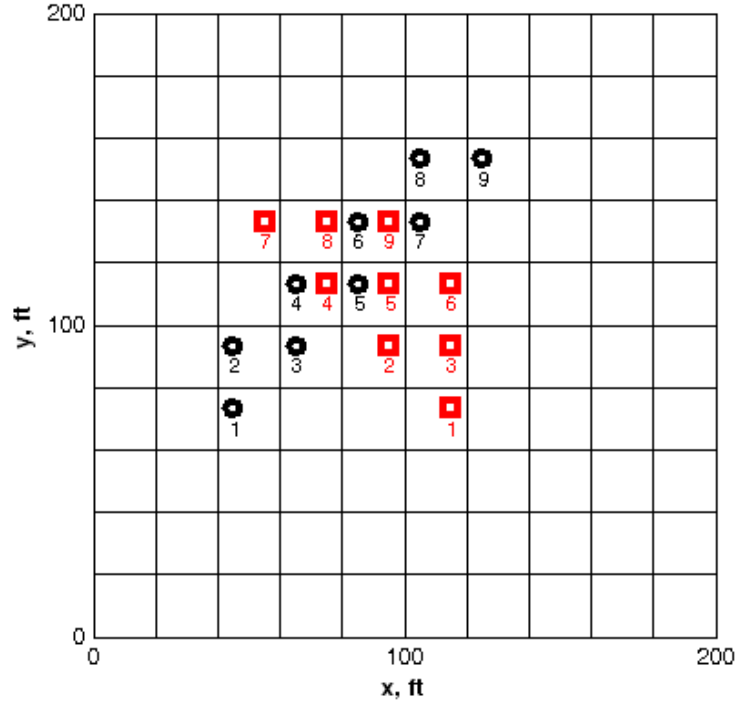

(b)

Figure 4.4: Specification of gridblocks containing a segment of a fracture for (a) layer 1, and (b) layer 2 in the $\mathrm{z}$ direction of the model shown in Figure 4.3. Cells containing a segment of the black fracture are marked with black circles and those containing a segment of the red fracture are marked with red squares. 
Figure 4.5 illustrates the arrangement of fracture cells in the fracture domain for the model shown in Figure 4.3. Fracture cells are marked and numbered in Figure 4.5, which correspond to those in Figure 4.4. A large number of unmarked cells in the fracture domain are treated as dead blocks in flow simulations to avoid increased computational time. The blue and green dashed lines in Figure 4.5 are described in a subsequent section.

A gridblock may contain more than one fracture segment, all of which should be considered separately in the fracture domain. For instance, the gridblock $(5,5)$ in the top layer contains segments of both fractures and thus, two fracture control volumes are correspondingly defined in gridblocks $(5,2)$ and $(6,3)$ of the top layer of fracture domain.

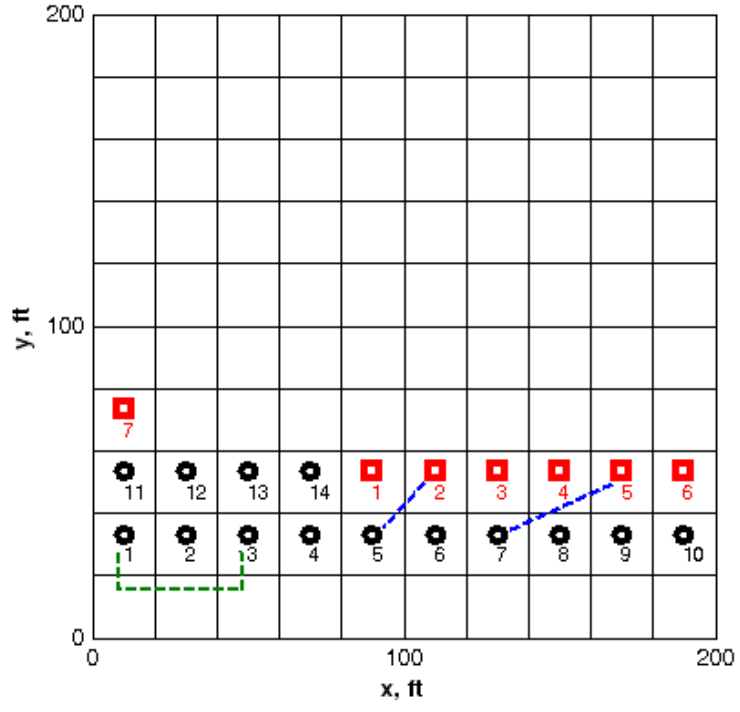

(a)

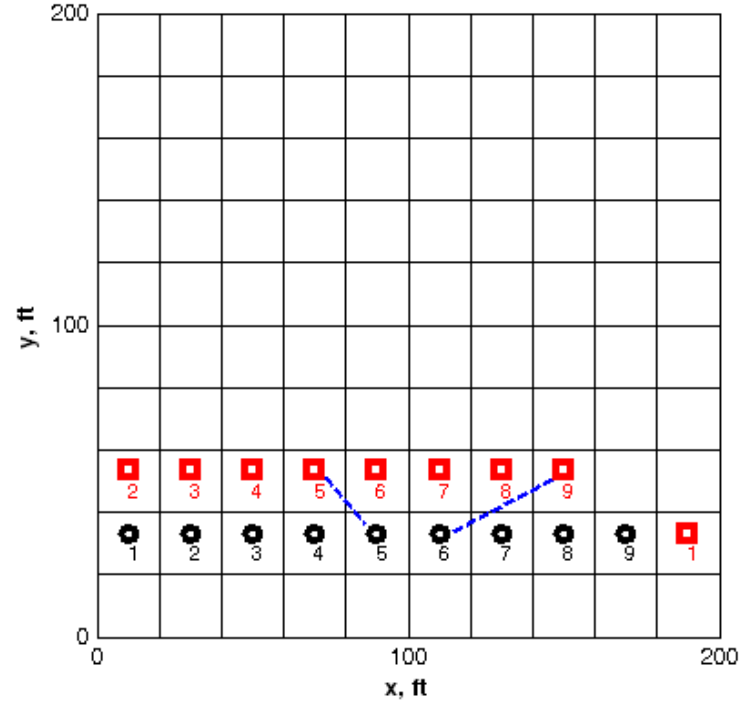

(b)

Figure 4.5: Arrangement of fracture cells in the fracture domain for (a) layer 1, and (b) layer 2 of the model shown in Figure 4.3. Fracture cells are marked and numbered in this figure, which correspond to those in Figure 4.4. The blue dashed lines represent non-neighboring connections (NNC) for the intersection of two fractures. Also, the green dashed line represents only one example of NNC between two cells of an individual fracture. 


\subsubsection{Non-Neighboring Connection (NNC)}

Because the fractures and the matrix are modeled on different computational domains, there is no fluid communication between them in the mass balance equations. Consequently, we define non-neighboring connections (NNC) for EDFM. That is, each gridblock in the numerical model can communicate with any other gridblock through a non-neighboring connection. The concept of NNC has been used in previous reservoir simulation studies, such as Fung and Dogru (2008). Also, Karimi-Fard et al. (2004) and Hui and Mallison (2009) used NNCs in their DFM approaches. Three types of NNCs are required in our fracture modeling:

- Non-neighboring connection between a fracture cell and its neighboring matrix gridblock. Matrix-fracture NNCs are marked and numbered correspondingly in Figures 4.4 and 4.5 as previously mentioned.

- Non-neighboring connection between two intersecting fractures. As shown in Figure 4.4, six gridblocks in the model contain segments of both fractures, but the intersection line of two fractures is located in four gridblocks. Thus, corresponding fracture cells should communicate with each other through a nonneighboring connection. This type of NNC is depicted in Figure 4.5 using blue dashed lines.

- Non-neighboring connection between two cells of an individual fracture if needed. This type of non-neighboring connection is a result of our fracture arrangement. For instance, fracture segments located in gridblocks $(3,4)$ and $(3,5)$ of Figure 4.4a should communicate with each other but they are not computational neighbors in the fracture domain; thereby, a non-neighboring connection is needed between them. The green dashed line in Figure 4.5a represents such a NNC. 
For the purpose of illustration, we consider a fracture cell marked with a black circle, numbered 7 in Figure 4.5a. This fracture cell, which is embedded in the corresponding matrix cell in Figure 4.4a, entails all three types of NNCs as shown in Figure 4.6.

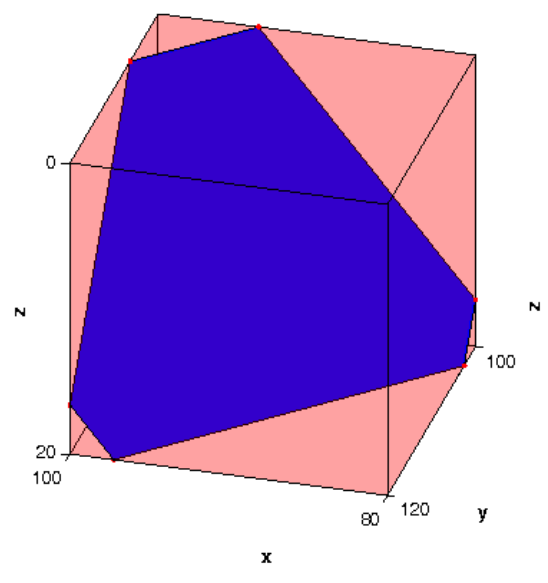

(a)

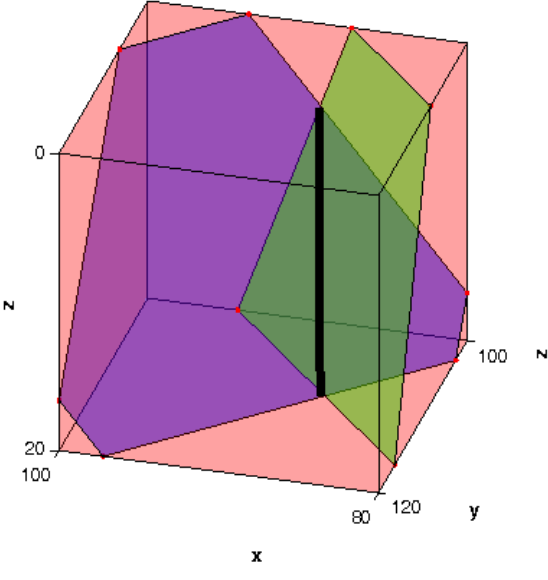

(b)

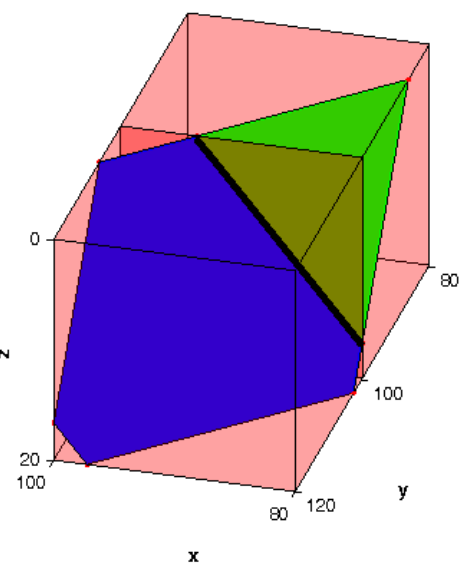

(c)

Figure 4.6: Three types of non-neighboring connections are required in the computational domain. (a) When a fracture segment is embedded in a gridblock, there is a NNC between the fracture control volume and the matrix cell. (b) When two fracture planes intersect in a gridblock, there is a NNC between corresponding fracture control volumes. The black solid line shows the intersection line bounded in the gridblock. In a later section, this length will be used to calculate the transmissibility between two intersecting fractures. (c) When two fracture segments embedded in neighboring cells are not neighbors in the computational domain, there is a NNC between corresponding fracture control volumes. The black solid line is the intersection line of the fracture plane and the common face of two neighboring gridblocks. 


\subsubsection{NNC Formulation}

Owing to the necessity of using NNCs for the EDFM approach, NNCs are added to GPAS in the component mass balance equation (Eq. 3.1) as follows:

$$
V_{b} \frac{\partial}{\partial t}\left(\phi N_{i}\right)-V_{b} \nabla \cdot \sum_{j=1}^{N_{p}} \frac{\overline{\bar{k}} k_{r j}}{\mu_{j}} \varepsilon_{j} x_{i j}\left(\vec{\nabla} P_{j}-\gamma_{j} \vec{\nabla} D\right)-q_{i}+q_{i}^{n n c}=0,
$$

where $q_{i}^{n n c}$ is the molar rate of component $i$ exchanged through NNCs. This term is mathematically similar to the convection term and is given by

$$
q_{i}^{n n c}=\sum_{m=1}^{N_{n n c}} A_{m}^{n n c} \sum_{j=1}^{n_{p}} \frac{k_{m}^{n n c} k_{r j}}{\mu_{j}} \varepsilon_{j} x_{i j}\left[\frac{\left(P_{j}-\gamma_{j} D\right)-\left(P_{j}-\gamma_{j} D\right)_{m}^{n n c}}{d_{m}^{n n c}}\right] \text {, }
$$

where $N_{n n c}$ is the number of non-neighboring connections for a gridblock and $\left(P_{j}-\right.$ $\left.\gamma_{j} D\right)^{n n c}$ represents the flow potential at the non-neighboring cell. For the fracture control volumes in the fracture domain $N_{n n c}$ is always greater than or equal to one, since each fracture cell has at least one non-neighboring connection with the matrix gridblock in which the fracture segment is embedded. Likewise, in the matrix domain, $N_{n n c}$ is simply equal to the number of fractures passing through a matrix gridblock. Hence, $N_{n n c}$ is zero for a matrix cell that does not contain any segment of a fracture.

The parameters $A^{n n c}, k^{n n c}$, and $d^{n n c}$ in Equation 4.2 are the area, permeability, and distance, respectively, used to determine the transmissibility factor between a NNC pair. More precisely, $A^{n n c}, k^{n n c}$, and $d^{n n c}$ are the area open to flow, the harmonic average of permeability, and the characteristic distance, respectively, between two control volumes associated with a NNC. For the mobility term in Equation 4.2, the classical single-point upstream weighting is used. 
The transmissibility factor $\left(A^{n n c} \times k^{n n c}\right) / d^{n n c}$ for the three types of NNCs previously described must be calculated and saved for each NNC. The next sections describe how the transmissibilities are calculated.

\subsubsection{NNC Type I}

For a NNC between matrix and fracture cells (Figure 4.6a), $A^{n n c}$ is the fracture surface area in the gridblock. Fluid transfer between the fracture and matrix gridblock takes place through this surface and an exact specification of the fracture-gridblock intersection is necessary to accurately calculate the area of this surface. The parameter $k^{n n c}$ is taken as the harmonic average of the matrix and fracture permeabilities. Therefore, $k^{n n c}$ is close to the matrix permeability in most cases where fracture permeability is significantly greater than matrix permeability.

To calculate $d^{n n c}$, Li and Lee (2008) and Hajibeygi et al. (2011) assumed that the

pressure varies linearly in the normal direction to each fracture in a gridblock and proposed the following equation for computing the average normal distance $(\langle d\rangle)$ :

$$
<d>=d^{n n c}=\frac{\int_{V} x_{n} d v}{V}
$$

where $d v, x_{n}$, and $V$ are the volume element, the normal distance of the element from the fracture, and volume of a gridblock, respectively. We calculate this integral numerically in a pre-processing code. Analytical expressions, however, exist for simple cases. Analytical expressions for some simple 2D scenarios are presenetd in Figure 4.7. Figure 4.8 also shows examples of more complex 3D scenarios calculated numerically using our pre-processing code. 

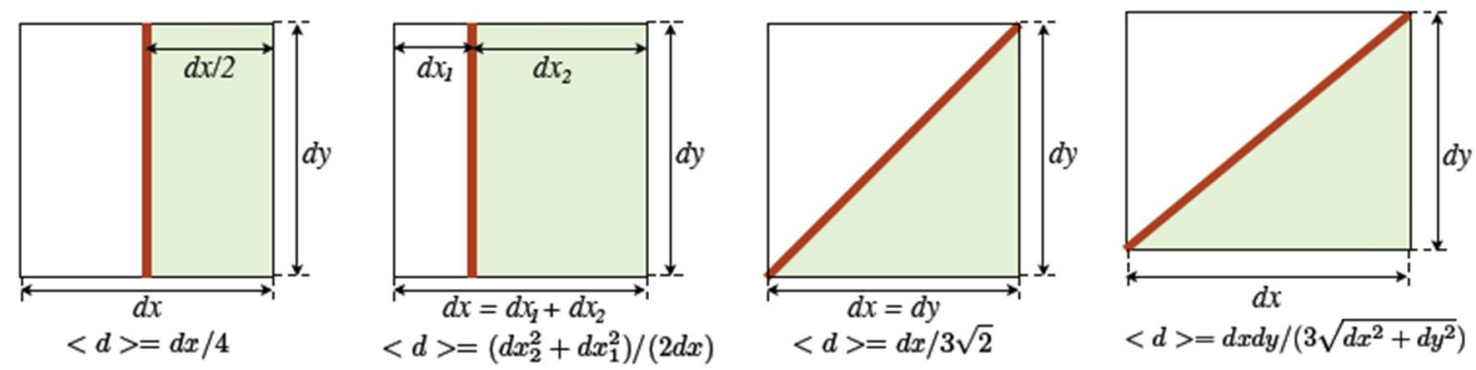

Figure 4.7: Analytical expressions of average normal distance between a matrix gridblock and an embedded fracture for some simple 2D scenarios (from Hajibeygi et al., 2011)

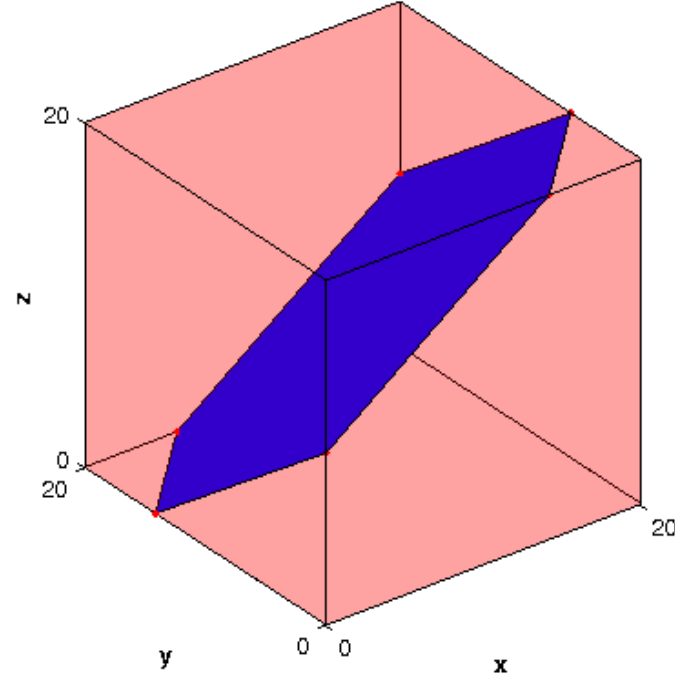

(a) $\langle d\rangle=4.70$

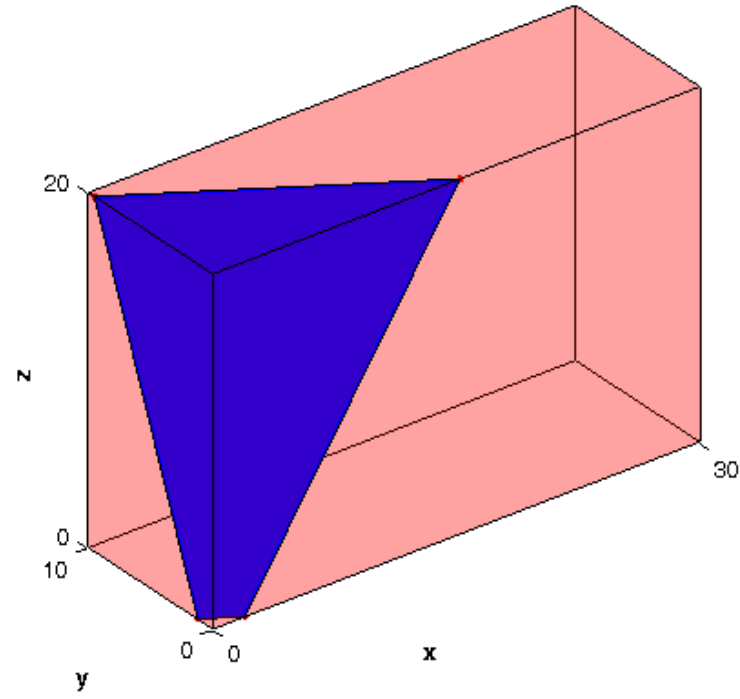

(b) $\langle d\rangle=7.55$

Figure 4.8: Examples of average normal distance between a matrix cell and its embedded fracture for complex 3D scenarios. (a) Normal vector of the fracture plane is $(-0.5,0.5, \sqrt{ } 2 / 2)$ and the fracture plane passes through the point $(10,10,10)$. (b) Normal vector of the fracture plane is $(0.5,0.8,-0.3317)$ and fracture plane passes through the point $(7,1,10)$. 


\subsubsection{NNC Type II}

For a NNC between two intersecting fracture segments, we use the same approach presented by Karimi-Fard et al. (2004), wherein the transmissibility is calculated by

$$
\begin{aligned}
& \frac{k^{n n c} A^{n n c}}{d^{n n c}}=\frac{T_{1} T_{2}}{T_{1}+T_{2}}, \\
& T_{1}=\frac{k_{f 1} \omega_{f 1} L_{i n t}}{d_{f 1}}, T_{2}=\frac{k_{f 2} \omega_{f 2} L_{i n t}}{d_{f 2}},
\end{aligned}
$$

where $L_{\text {int }}$ is the length of the intersection line bounded in a gridblock (black solid line in Figure 4.6b). Also, $\omega_{f}$ and $k_{f}$ are fracture aperture and fracture permeability, respectively. Likewise, $d_{f}$ is the average of normal distances from the center of the fracture subsegments (located in each side of the intersection line) to the intersection line.

Such a NNC is required for any pair of intersecting fractures. Thus, if more than two fractures intersect in a gridblock, a NNC is defined between each pair of intersecting fracture control volumes. Also, if two fractures penetrating a gridblock do not intersect with each other within the gridblock, no NNC is needed.

\subsubsection{NNC Type III}

For a NNC between two cells of an individual fracture, $k^{n n c}$ is equal to the fracture permeability and $d^{n n c}$ is the distance between the centers of two fracture segments. The black solid line in Figure 4.6c represents the intersection line of the fracture plane and the common face of two neighboring gridblocks. Parameter $A^{n n c}$ is the fracture aperture times the length of this intersection line. 


\subsubsection{Transmissibility of Neighboring Fracture Control Volumes}

As described in Chapter 3, the IPARS framework calculates the constant part of transmissibility between adjacent cells in all directions based on the geometry and the permeability of gridblocks. In implementing the EDFM approach, the fracture control volumes defined in the fracture domain do not represent the realistic location and geometry of fractures. Hence, the transmissibility between adjacent fracture control volumes should be computed in a pre-processing code rather than in the IPARS framework.

For illustration, consider the fracture cell marked with a black circle and numbered 1 in Figure 4.5a. This fracture cell should communicate with the fracture cell numbered 2 since the corresponding fracture segments are connected in the actual reservoir model (see Figure 4.4a). Such a communication is maintained through a proper transmissibility factor in the $\mathrm{x}$ direction in the fracture domain. However, the fracture cell numbered 1 should not communicate with the one numbered 11 although they are adjacent in the fracture domain, because the corresponding fracture segments are not directly connected in the actual model (see Figure 4.4a). Hence, the transmissibility in the y direction (between these two fracture cells) should be set to zero.

We need to properly calculate all the transmissibility factors in the $\mathrm{x}, \mathrm{y}$, and $\mathrm{z}$ directions for fracture control volumes in a pre-processing code. These factors are then used in the reservoir simulator over the course of simulation. We use the same approach described for the NNC type III to compute the transmissibility between fracture cells $\left(T_{f}\right)$, which is given by

$$
T_{f}=\frac{k_{f} \omega_{f} L_{f}}{d_{f}},
$$


where $k_{f}$ and $\omega_{f}$ are the fracture permeability and fracture aperture, respectively. Likewise, $d_{f}$ is the distance between the centers of two fracture segments, and $L_{f}$ is the length of the intersection line of the fracture plane and the common face of two neighboring gridblocks.

\subsubsection{Well and Fracture Intersection}

An accurate well model is required to relate the well rate to the well pressure and the pressure of fracture intersecting the well. This intersection has the most significant influence on well productivity. Peaceman (1983) established a mathematical model between the well block pressure and the wellbore pressure for a vertical well. The Peaceman's well index $(W I)$ for a vertical well, which is used in most reservoir simulators, is given by

$$
\begin{aligned}
& W I=\frac{k h}{\ln \left(\frac{r_{0}}{r_{w}}\right)}, \\
& r_{0}=0.28 \frac{\sqrt{\left(k_{y} / k_{x}\right)^{0.5} \Delta x^{2}+\left(k_{x} / k_{y}\right)^{0.5} \Delta y^{2}}}{\left(k_{y} / k_{x}\right)^{0.25}+\left(k_{x} / k_{y}\right)^{0.25}},
\end{aligned}
$$

where $k_{x}$ and $k_{y}$ are the permeability in the $\mathrm{x}$ and $\mathrm{y}$ directions, respectively, and $\Delta x$ and $\Delta y$ are the horizontal dimensions of the well block. Also, $k, h$, and $r_{w}$ are the well block permeability, the well block height (identical to the length of well in the gridblock), and the wellbore radius, respectively.

In EDFM, depending on well-fracture geometric configuration, we adapt the Peaceman's well model to derive a relationship for the well-fracture intersection. For instance, when a transverse hydraulic fracture intersects a horizontal well in a gridblock, 
the well index of a fracture, that relates the wellbore pressure to the pressure of the corresponding fracture control volume, is approximated by (assuming isotropic fracture permeability)

$$
\begin{aligned}
& W I_{f}=\frac{k_{f} \omega_{f}}{\ln \left(\frac{r_{0}}{r_{w}}\right)}, \\
& r_{0}=0.14 \sqrt{L_{f}{ }^{2}+h_{f}^{2}},
\end{aligned}
$$

where $k_{f}$ is the fracture permeability, $\omega_{f}$ is the fracture aperture (equal to the length of the well intercepted by a transverse fracture), $L_{f}$ is the fracture length bounded in the gridblock, and $h_{f}$ is the fracture height in the same block.

\subsection{Pre-Processing Code}

We have developed a pre-processing code to provide the required data for fluidflow simulations in GPAS. The input of the pre-processing code is the description of the model reservoir including the reservoir dimensions, fracture network, location of wells, structured grid for the matrix domain, aperture and permeability of fractures, and porosity and permeability of matrix. The following calculations are carried out in the preprocessing code:

- Check the intersection of each fracture plane with all matrix gridblocks and determine the exact specification of intersections.

- Check if any two fracture planes intersect and identify the gridblocks in which the intersection lies.

- Determine the arrangement of fracture control volumes in the fracture domain. Corresponding to each gridblock containing a segment of a fracture, a fracture 
control volume should be defined in the fracture domain. Figure 4.5 presented an example of fracture arrangement for a simple 3D model reservoir. Furthermore, arrangement of fracture cells leads to the identification of dead blocks.

- Calculate the porosity of fracture control volumes in the fracture domain. The porosity of each fracture control volume is the volume of the corresponding fracture segment bounded in a gridblock divided by the bulk volume of the gridblock. Moreover, the permeability of a fracture control volume is equal to the permeability of the corresponding fracture segment.

- Calculate the depth associated with each fracture control volume. Depth of each fracture cell is equal to the depth of fracture segment midpoint. Implementing the depth of fracture control volumes in the mass balance equation (Equations 4.2 and 4.3) will take into account the effect of gravity within vertical and non-vertical fractures.

- Calculate the number of NNCs for each computational gridblock either in the matrix domain or in the fracture domain.

- Prepare a list of NNC pairs. This list includes all three types of NNCs used in the EDFM approach.

- Calculate the transmissibility between each NNC pair using the methods presented earlier.

- Calculate the transmissibility factors in the $\mathrm{x}, \mathrm{y}$, and $\mathrm{z}$ directions for the fracture control volumes in the fracture domain.

- Identify fracture segments intersected by a well and calculate the well index of the corresponding fracture control volumes. 
The entire model, including the matrix grid and fracture control volumes, is entered into a reservoir simulator, which allows for non-neighboring connections and transmissibility modifiers. The governing equations for fracture control volumes are similar to those described for the matrix medium, implying that Darcy's law is used in the fracture domain.

In order to implement the EDFM approach in GPAS, we have added the NNC term to the mass balance equations, as previously described in Equations 4.2 and 4.3. In doing so, the residual vector and the Jacobian matrix (see Chapter 3) are augmented with additional terms associated with NNCs. Other modifications to GPAS have also been implemented to properly include the parameters calculated in the pre-processing code. These parameters include porosity, permeability, and depth of fracture control volumes, transmissibility factors between NNCs, transmissibility factors between adjacent fracture cells, and well indices for the fractures intercepted by a well. Also, we have made necessary changes to bypass all calculations carried out for the dead blocks in the fracture domain.

The addition of the NNC term to the mass balance equations affects the sparsity and location of zeros in the Jacobian matrix and reduces the sparseness of the linear system that must be solved. The EDFM approach may require excessive NNCs and consequently may affect the performance of the linear solvers used in the simulator. However, in several case studies presented in the forthcoming chapters, the performance of the linear solver, PETSc package (Balay et al., 1998), was not significantly diminished by adding excessive NNCs. 


\subsubsection{Example Calculation}

This section presents an example calculation including parameters calculated in the pre-processing code. For illustration purposes, the example is a $2 \mathrm{D}$ reservoir containing 14 vertical fractures as shown in Figure 4.9a. Simulations performed for this model will be presented in Chapter 6 . Reservoir dimensions are $500 \times 500 \times 20 \mathrm{ft}$ and Table 4.1 describes the location of fractures using two endpoints, (X1, Y1) and (X2, Y2). The matrix grid is $20 \times 20 \times 1$, where the sizes of each gridblock in the $\mathrm{x}, \mathrm{y}$, and $\mathrm{z}$ directions are 25,25 , and $20 \mathrm{ft}$, respectively. The aperture and permeability of all fractures are $0.025 \mathrm{ft}$ and $7 \times 10^{5} \mathrm{md}$, respectively. Also, porosity and permeability of the matrix are 0.1 and 20 md.

Figure $4.9 \mathrm{~b}$ shows the arrangement of fracture control volumes in the fracture domain, which are marked with the same color as their corresponding fracture in Figure 4.9a. In our implementation we do not place the fracture control volumes in the first and last rows in y direction. This is because of the future implementation of EDFM for parallel processing, wherein the reservoir domain is divided in the y direction into several subdomains and the ghost layers maintain communication between processors (see Chapter 3). Also, blue dashed lines in Figure 4.9b show the NNCs associated with intersection of fractures (NNC type II).

Table 4.2 presents only a portion of the calculated data in the pre-processing code for the model reservoir shown in Figure 4.9a. That is, only the relevant data associated with the first three fractures (as defined in Table 4.1 or depicted in Figure 4.9b) are presented in Table 4.2. The first column in this table counts the cell number of fracture control volumes in the fracture domain. This column starts with 21 owing to the absence of fracture cells in the first row. The cells are numbered sequentially, first in the $\mathrm{x}$ direction and then in the y direction. The second column of Table 4.2 presents the 
porosity assigned to each fracture control volume. The third column shows the cell number of matrix gridblocks that are connected to the fracture cells through NNCs type I. The fourth column presents the transmissibility of NNCs between corresponding matrix and fracture cells. When a fracture does not fully penetrate a gridblock, we first calculate the transmissibility assuming that the fracture fully penetrates the gridblock. Then, we correct the transmissibility for the partially-penetrating fracture (transmissibility is proportional to the fracture area bounded in the gridblock). The fifth column in Table 4.2 shows the computed transmissibility factors between adjacent fracture cells in the $\mathrm{x}$ direction. Furthermore, Table 4.3 presents the NNCs type II and type III for the reservoir shown in Figure 4.9. Both ends of NNCs type II and type III are located in the fracture domain. Table 4.3 includes the cell number of NNC pairs in conjunction with the transmissibility assigned to them.

\begin{tabular}{|c|c|c|c|c|}
\hline Fracture & X1 $(\mathrm{ft})$ & Y1 $(\mathrm{ft})$ & $\mathbf{X 2}(\mathrm{ft})$ & Y2 $(\mathrm{ft})$ \\
\hline 1 & 117.5 & 37.5 & 285 & 210 \\
\hline 2 & 396 & 251.5 & 206 & 336.5 \\
\hline 3 & 352.5 & 241 & 445 & 434 \\
\hline 4 & 297 & 83 & 128.5 & 246 \\
\hline 5 & 201.5 & 461.5 & 366.5 & 387 \\
\hline 6 & 297 & 351 & 401 & 454.5 \\
\hline 7 & 127 & 203.5 & 243.5 & 476.5 \\
\hline 8 & 211.5 & 101.5 & 436 & 211.5 \\
\hline 9 & 451.5 & 34 & 329.5 & 213.5 \\
\hline 10 & 44.5 & 411.5 & 181.5 & 285.5 \\
\hline 11 & 360.5 & 29.5 & 460.5 & 93.5 \\
\hline 12 & 42 & 120.5 & 87.5 & 231 \\
\hline 13 & 42 & 312 & 94.5 & 438.5 \\
\hline 14 & 36.5 & 37.5 & 160 & 70 \\
\hline
\end{tabular}

Table 4.1: Fracture endpoints for the model reservoir shown in Figure 4.9a. 


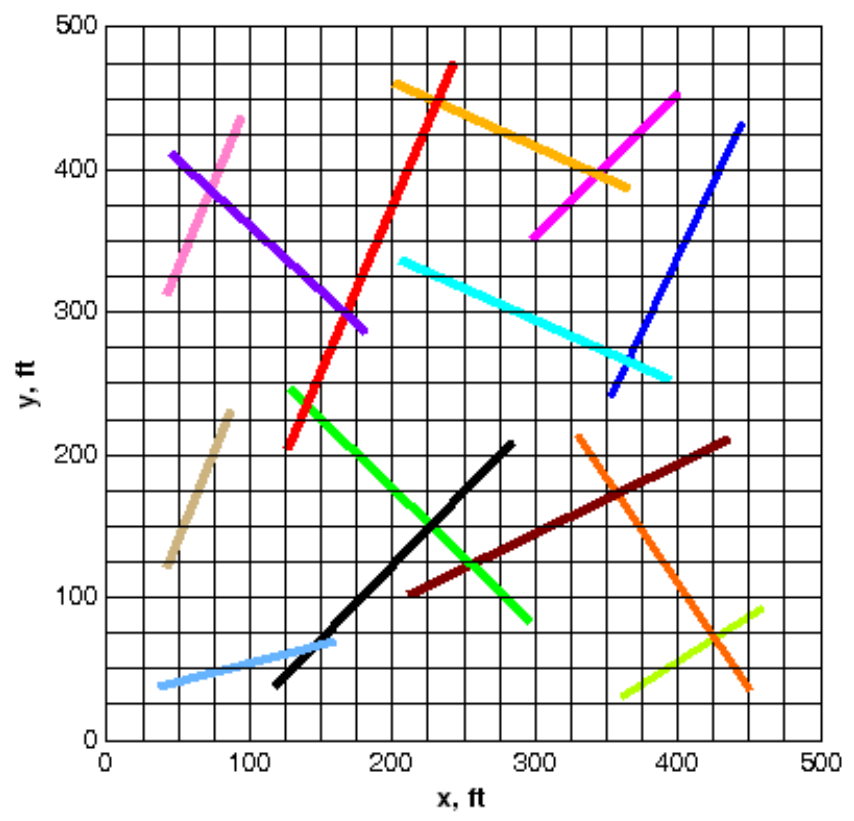

(a)

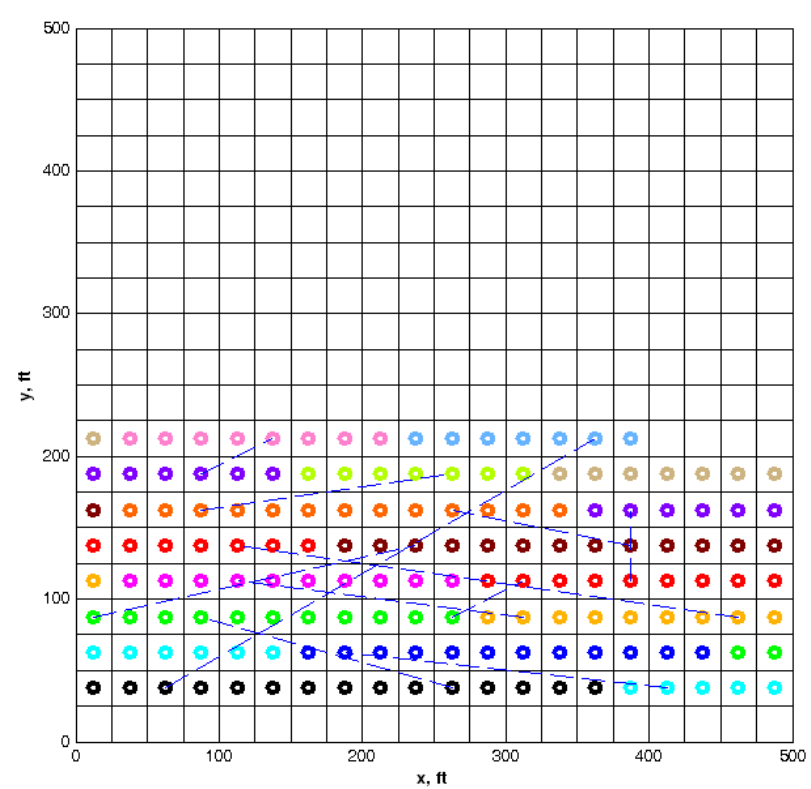

(b)

Figure 4.9: (a) A synthetic 2D model containing 14 fractures. (b) Arrangement of fracture control volumes in the fracture domain, which are marked with the same color as their corresponding fracture in Figure 4.9a. Also, blue dashed lines show the NNCs associated with intersection of fractures (NNC type II). 


\begin{tabular}{|c|c|c|c|c|}
\hline $\begin{array}{l}\text { Fracture } \\
\text { cell no. }\end{array}$ & $\begin{array}{l}\text { Fracture } \\
\text { porosity }\end{array}$ & $\begin{array}{l}\text { Matrix cell no. } \\
\text { (NNC type } 1)\end{array}$ & $\begin{array}{c}\text { NNC } \\
\text { Transmissibility }\end{array}$ & $\begin{array}{l}\text { x-transmissibility } \\
\text { between } G_{f} \text { and } G_{f-1}\end{array}$ \\
\hline$G_{f}$ & $\phi_{f}$ & $G_{m}$ & $\boldsymbol{T}_{\boldsymbol{n} n \boldsymbol{c}}(\mathrm{md} . \mathrm{ft})$ & $\boldsymbol{T}_{\boldsymbol{x}}$ (md.ft) \\
\hline 21 & 0.000431 & 25 & 655.34 & ----- \\
\hline 22 & 0.000266 & 26 & 185.08 & 40175.96 \\
\hline 23 & 0.001169 & 46 & 1828.53 & 19505.72 \\
\hline 24 & 0.000225 & 47 & 150.87 & 20087.98 \\
\hline 25 & 0.001211 & 67 & 1939.69 & 19505.72 \\
\hline 26 & 0.000183 & 68 & 118.87 & 20087.98 \\
\hline 27 & 0.001252 & 88 & 2047.59 & 19505.72 \\
\hline 28 & 0.000141 & 89 & 88.89 & 20087.98 \\
\hline 29 & 0.001294 & 109 & 2150.29 & 19505.72 \\
\hline 30 & 0.000100 & 110 & 60.78 & 20087.98 \\
\hline 31 & 0.001336 & 130 & 2245.81 & 19505.72 \\
\hline 32 & 0.000058 & 131 & 34.37 & 20087.98 \\
\hline 33 & 0.001377 & 151 & 2332.16 & 19505.72 \\
\hline 34 & 0.000017 & 152 & 9.53 & 20087.98 \\
\hline 35 & 0.000558 & 172 & 946.07 & 48764.30 \\
\hline 36 & 0.000920 & 216 & 1153.58 & 0 \\
\hline 37 & 0.001095 & 215 & 1643.41 & 13890.71 \\
\hline 38 & 0.000286 & 214 & 206.20 & 20265.93 \\
\hline 39 & 0.000809 & 234 & 844.34 & 25558.91 \\
\hline 40 & 0.001095 & 233 & 1780.51 & 14698.97 \\
\hline 41 & 0.000544 & 232 & 467.56 & ----- \\
\hline 42 & 0.000552 & 252 & 476.83 & 25558.91 \\
\hline 43 & 0.001095 & 251 & 1782.71 & 16999.28 \\
\hline 44 & 0.000802 & 250 & 831.53 & 14758.88 \\
\hline 45 & 0.000294 & 270 & 212.85 & 25558.91 \\
\hline 46 & 0.000833 & 269 & 1253.27 & 24857.06 \\
\hline 47 & 0.000399 & 195 & 368.01 & 0 \\
\hline 48 & 0.001109 & 215 & 1826.59 & 18566.02 \\
\hline 49 & 0.000574 & 235 & 507.32 & 16635.36 \\
\hline 50 & 0.000535 & 236 & 458.62 & 25249.79 \\
\hline 51 & 0.001109 & 256 & 1821.79 & 17035.78 \\
\hline 52 & 0.000670 & 276 & 636.66 & 15738.58 \\
\hline 53 & 0.000439 & 277 & 350.84 & 25249.79 \\
\hline 54 & 0.001109 & 297 & 1794.25 & 18091.44 \\
\hline 55 & 0.000766 & 317 & 783.32 & 14933.54 \\
\hline 56 & 0.000343 & 318 & 256.16 & 25249.79 \\
\hline 57 & 0.001109 & 338 & 1748.11 & 19286.58 \\
\hline 58 & 0.000399 & 358 & 439.64 & 18566.02 \\
\hline
\end{tabular}

Table 4.2: A portion of the calculated data for the fractures in Figure 4.9. 


\begin{tabular}{|c|c|c|c|}
\hline NNC end 1 & NNC end 2 & NNC type & NNC Transmissibility \\
\hline $\boldsymbol{G}_{\boldsymbol{f} \boldsymbol{1}}$ & $\boldsymbol{G}_{\boldsymbol{f} \boldsymbol{2}}$ & ---- & $\boldsymbol{T}_{\boldsymbol{n n \boldsymbol { c }}}(\mathrm{md} . \mathrm{ft})$ \\
\hline 40 & 41 & III & 17079.46 \\
\hline 60 & 61 & III & 19467.82 \\
\hline 80 & 81 & III & 25048.60 \\
\hline 100 & 101 & III & 25753.11 \\
\hline 120 & 121 & III & 26782.55 \\
\hline 140 & 141 & III & 20609.07 \\
\hline 160 & 161 & III & 21321.99 \\
\hline 23 & 175 & II & 25417.21 \\
\hline 31 & 64 & II & 37632.75 \\
\hline 37 & 48 & II & 25403.41 \\
\hline 61 & 110 & II & 34709.91 \\
\hline 71 & 93 & II & 24520.68 \\
\hline 73 & 85 & II & 32222.45 \\
\hline 79 & 105 & II & 32116.61 \\
\hline 96 & 136 & II & 35202.38 \\
\hline 116 & 131 & II & 32526.68 \\
\hline 124 & 151 & II & 39297.52 \\
\hline 144 & 166 & II & 33073.55 \\
\hline
\end{tabular}

Table 4.3: NNCs type II and type III for the reservoir shown in Figure 4.9. The cell number of NNC pairs and the transmissibility assigned to them are presented in this table. 


\subsection{Highlights OF THE CHAPTER}

- An embedded discrete fracture model (EDFM) is developed for our in-house fully-implicit compositional reservoir simulator (GPAS).

- The work performed in this study is an extension of an algorithm developed by Li and Lee (2008). The extended algorithm includes slanted fractures of any orientation, honoring the complexity and heterogeneity of a typical fractured reservoir.

- EDFM borrows the dual medium concept from dual continuum models, but also incorporates the effect of each fracture explicitly.

- EDFM employs a structured grid to surmount challenges associated with unstructured gridding.

- EDFM is based on non-neighboring connections (NNC). Three types of NNCs are required for modeling vertical and slanted fractures. It is necessary to properly calculate the transmissibility between NNCs and the transmissibility between fracture cells.

- The EDFM approach is compatible with reservoir simulators that allow for NNCs and transmissibility modifiers (such as commercial reservoir simulators).

- The NNC term is added to the mass balance equations in the EOS compositional module of GPAS. 


\section{Chapter 5: Verification of Embedded Discrete Fracture Model}

In this chapter, we present simulation examples to verify the accuracy of the EDFM approach. First, we compare the results of our method with analytical solutions for multiply-fractured horizontal wells in bounded rectangular reservoirs. Then, the accuracy of the EDFM approach is confirmed by comparing the results with the fine-grid, explicit-fracture simulations for case studies including isolated fractures, intersected orthogonal fractures, and a non-orthogonal fracture, respectively. The analytical solutions are presented and compared with EDFM for primary production, while the fine-grid simulations are presented to confirm the accuracy of the EDFM approach for two-phase water flooding and three-phase compositional gas injection. We also perform a grid sensitivity study for the case study with a non-orthogonal fracture to show that EDFM can achieve accurate results using moderate grid refinement. Furthermore, we present the computational performance of EDFM simulations indicating that the developed approach is computationally efficient.

\subsection{COMParison to ANALYTICAL SOlutions}

This section compares the predictions of the EDFM approach with analytical solutions for multiply-fractured horizontal wells in bounded rectangular reservoirs. We tested EDFM for two production schemes, constant-pressure production and constant-rate production. Figures 5.1a and 5.2a show the geometrical configuration of reservoirs, horizontal wells, and fractures for the production scenarios mentioned above, respectively. In both cases, the horizontal well is parallel to the longer side of the 
drainage region and the center of the well coincides with that of the drainage region. Likewise, three equally-spaced transverse fractures in each case are parallel to the shorter side and penetrate the formation completely. We consider flow of a slightly-compressible liquid in a homogenous porous medium. Analytical solutions are from Chen and Raghavan (1997) presented in dimensionless forms. The dimensionless bottom-hole pressure $\left(P_{w D}\right)$, dimensionless rate $\left(q_{D}\right)$, and dimensionless time $\left(t_{D}\right)$ are

$$
\begin{aligned}
& P_{w D}\left(t_{D}\right)=\frac{k_{m} h}{141.2 q \mu B}\left[P_{i}-P_{w}(t)\right] \\
& q_{D}\left(t_{D}\right)=\frac{141.2 \mu B}{k_{m} h\left(P_{i}-P_{w}\right)} q(t) \\
& t_{D}=\frac{0.0002637 k_{m}}{\phi c \mu L^{2}} t
\end{aligned}
$$

where $P_{i}, P_{w}, q, t, k_{m}, h, \mu, B$, and $\phi$ are initial reservoir pressure [psi], bottom-hole pressure [psi], production rate [STB/day], time [hr], matrix permeability [md], formation thickness [ft], viscosity [cp], formation volume factor $[\mathrm{RB} / \mathrm{STB}]$, and porosity, respectively. The fracture length is denoted by $x_{f}$ and the distance between the two outermost fractures is denoted by $d$. The reference length $(L)$ in Equation 5.3 is equal to fracture half-length for a single-fracture system and equal to $d / 2$ for a multiple-fracture system. Moreover, the dimensionless fracture conductivity $\left(C_{F D}\right)$ is defined as

$$
C_{F D}=\frac{k_{f} \omega_{f}}{k_{m} x_{f}} \text {, }
$$

where $k_{f}$ and $\omega_{f}$ are the fracture permeability and fracture aperture. In both scenarios under consideration, the properties of all fractures are identical and chosen so that the dimensionless fracture conductivity is equal to one. Also, the ratio of the horizontal-well length to the length of each fracture is 10. For the EDFM simulations, the fracture 
aperture, fracture permeability, formation permeability, fracture length, and horizontalwell length are $0.03 \mathrm{ft}, 10000 \mathrm{md}, 0.3 \mathrm{md}, 200 \mathrm{ft}$, and $2000 \mathrm{ft}$, respectively. Since the solutions are in dimensionless form, there is no additional constraint on other properties used in the simulations.

Figures $5.1 \mathrm{~b}$ and $5.2 \mathrm{~b}$ compare the predictions of the EDFM approach with the analytical solutions. The log-log plot of dimensionless production rate versus dimensionless time in Figure 5.1b and the log-log plot of dimensionless wellbore pressure derivative versus dimensionless time in Figure 5.2b indicate that the EDFM simulations are in very good agreement with the analytical solutions.

For the EDFM simulation in the first scenario, we used an $11 \times 45 \times 1$ matrix grid. The computational time of EDFM simulation was 6 seconds. Likewise, for the second scenario we used a $31 \times 55 \times 1$ matrix grid and the EDFM simulation took 65 seconds. The descriptions of matrix grid for both cases are given in Table 5.1. All simulations for this study were performed using Petros cluster, which is owned by the Center for Petroleum and Geosystems Engineering (CPGE) at The University of Texas at Austin. This 64-bit Linux cluster has 32 compute nodes where each node has 16 GB memory and 4 CPUs with the frequency of $2.73 \mathrm{GHz}$.

\begin{tabular}{|c|l|l|}
\hline $\begin{array}{c}\text { Production } \\
\text { Scenario }\end{array}$ & \multicolumn{2}{|c|}{ Grid Description } \\
\hline $\begin{array}{c}\text { Constant-pressure } \\
\text { production }\end{array}$ & $\Delta \mathrm{x}, \mathrm{ft}$ & $4 \times 100,80,40,80,4 \times 100$ \\
\cline { 2 - 3 } & $\Delta \mathrm{y}, \mathrm{ft}$ & $4 \times 100,2 *(50,30,16,8,16,50,30,8 \times 100), 50,30,16,8,16,50,30,4 \times 100$ \\
\hline $\begin{array}{c}\text { Constant-rate } \\
\text { production }\end{array}$ & $\Delta \mathrm{x}, \mathrm{ft}$ & $14 \times 100,80,40,80,14 \times 100$ \\
\cline { 2 - 3 } & $\Delta \mathrm{y}, \mathrm{ft}$ & $9 \times 100,2 *(50,30,16,8,16,50,30,8 \times 100), 50,30,16,8,16,50,30,9 \times 100$ \\
\hline
\end{tabular}

Table 5.1: Grid description for the EDFM simulations performed for the models shown in Figures 5.1a and 5.2a. 


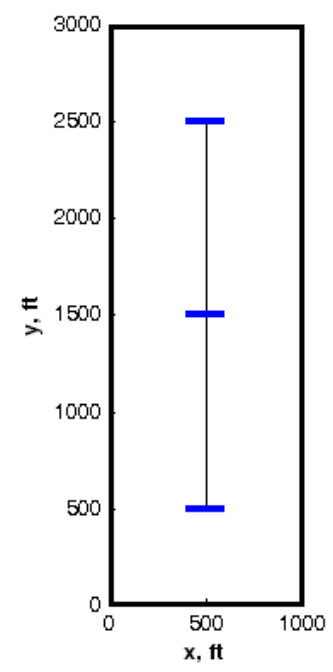

(a)

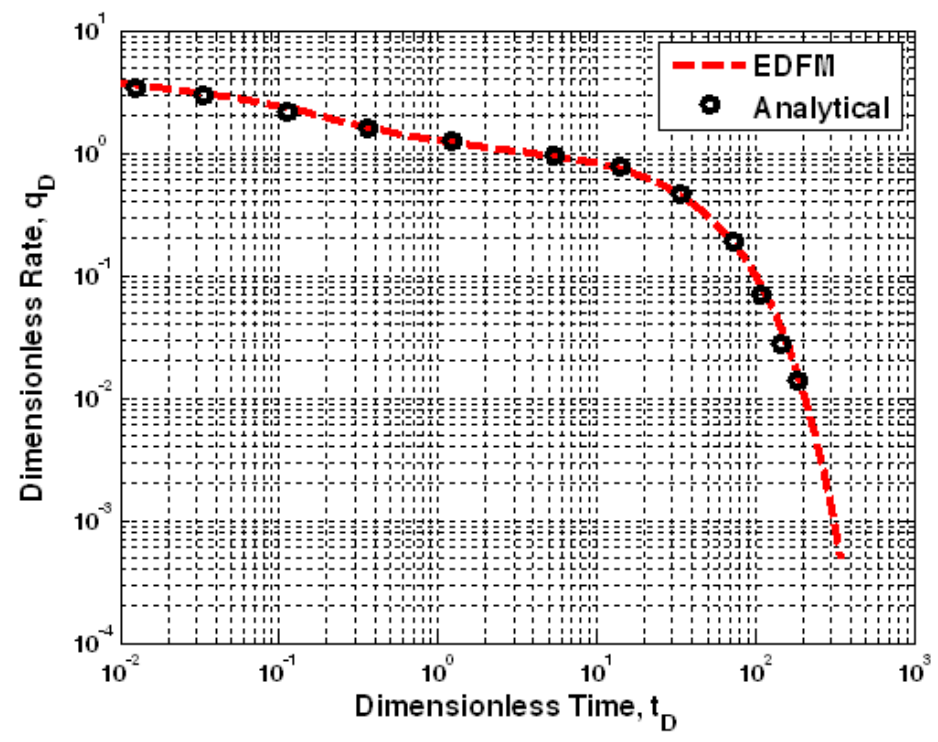

(b)

Figure 5.1: (a) Geometrical configuration of a reservoir with a multiply-fractured horizontal well producing at a constant bottom-hole pressure. (b) Comparison of dimensionless rate versus dimensionless time calculated by the EDFM simulation and analytical solutions (from Chen and Raghavan, 1997). 


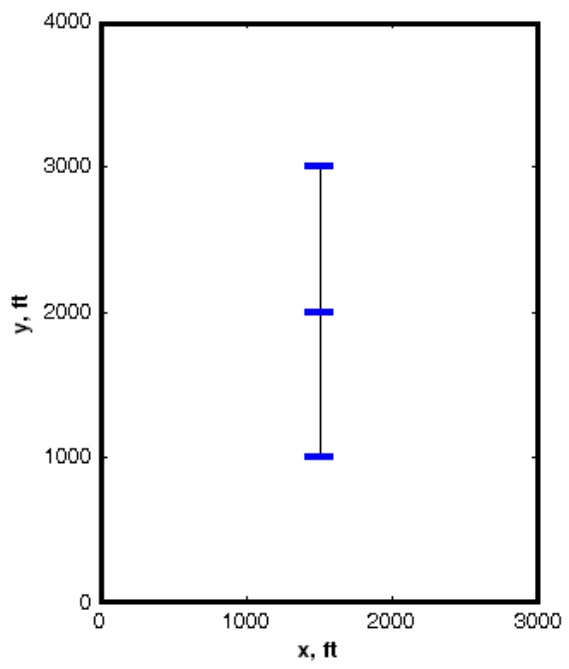

(a)

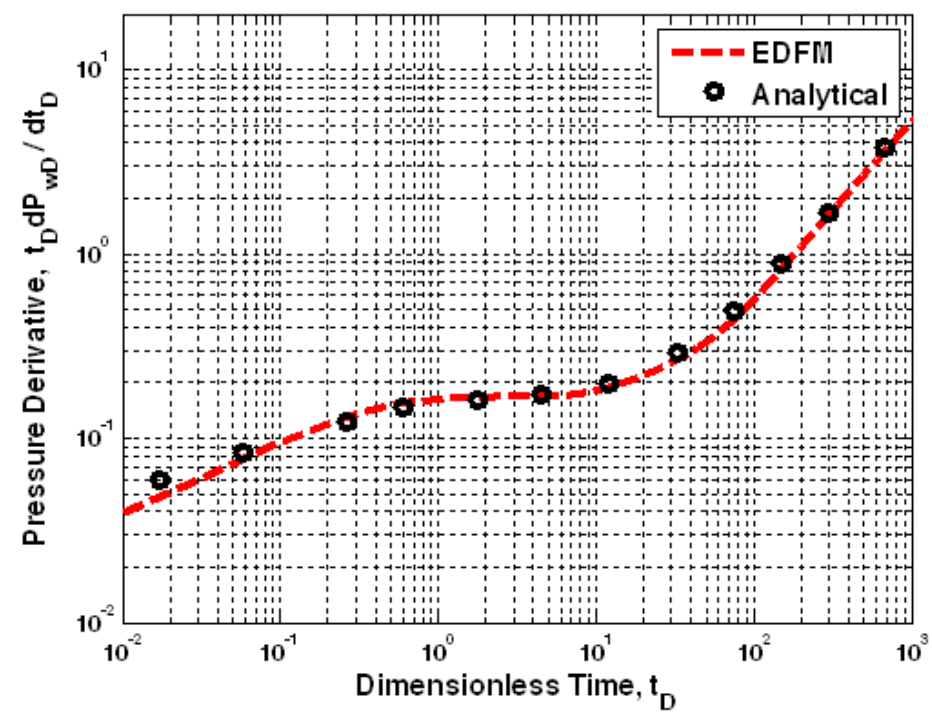

(b)

Figure 5.2: (a) Geometrical configuration of a reservoir with a multiply-fractured horizontal well producing at a constant rate. (b) Comparison of dimensionless wellbore pressure derivative versus dimensionless time calculated by the EDFM approach and analytical solutions (from Chen and Raghavan, 1997). 


\subsection{COMPARISON TO FINE GRID EXPLICIT FraCtURE SIMULATIONS}

In this section, we compare the EDFM approach with fine-grid, explicit-fracture models to investigate the accuracy and efficiency of the EDFM approach.

\subsubsection{Isolated Fractures}

Figure 5.3 shows a simple two-layer fractured reservoir containing two isolated fractures. Both fractures are vertical and aligned with the coordinate axes, thereby allowing us to perform standard finite-difference modeling. For the fine-grid simulation, the grid is $30 \times 55 \times 2$ cells in the $\mathrm{x}, \mathrm{y}$, and $\mathrm{z}$ directions respectively. The cell dimensions are $20 \times 15 \mathrm{ft}$ in $\mathrm{x}$ and $\mathrm{z}$, while they are non-uniform in $\mathrm{y}$ to accommodate refinement near fractures. For the EDFM simulation, a uniform $30 \times 15 \times 2$ matrix grid and 76 fracture cells are used. As shown in Figure 5.3, an injector is located in one side of the reservoir, injecting water at $250 \mathrm{bbl} / \mathrm{day}$, and a producer is placed in the opposite side. Initial reservoir pressure and the constant producer BHP are 5000 psi. Also, matrix and fracture capillary pressures are zero. We assume straight-line relative permeability curves (with zero residual saturations and endpoints equal to one) for fractures in all simulations of this work. However, there is no limitation to use other relative permeability curves for the fractures. The only hydrocarbon component in this example is $\mathrm{C}_{10} \mathrm{H}_{22}$. Table 5.2 summarizes petrophysical properties used in both simulations.

Water saturation and pressure profiles after 100 days of water injection (0.26 PV injected) calculated by EDFM and fine-grid explicit-fracture models are presented in Figure 5.4, indicating a very good agreement between both models. Furthermore, Figure 5.5 compares the oil production rate over a year ( $0.95 \mathrm{PV}$ injected) and confirms the accuracy of the EDFM approach. The computational times for the EDFM and fine-grid explicit-fracture models were 3.4 and 58.5 minutes, respectively. 


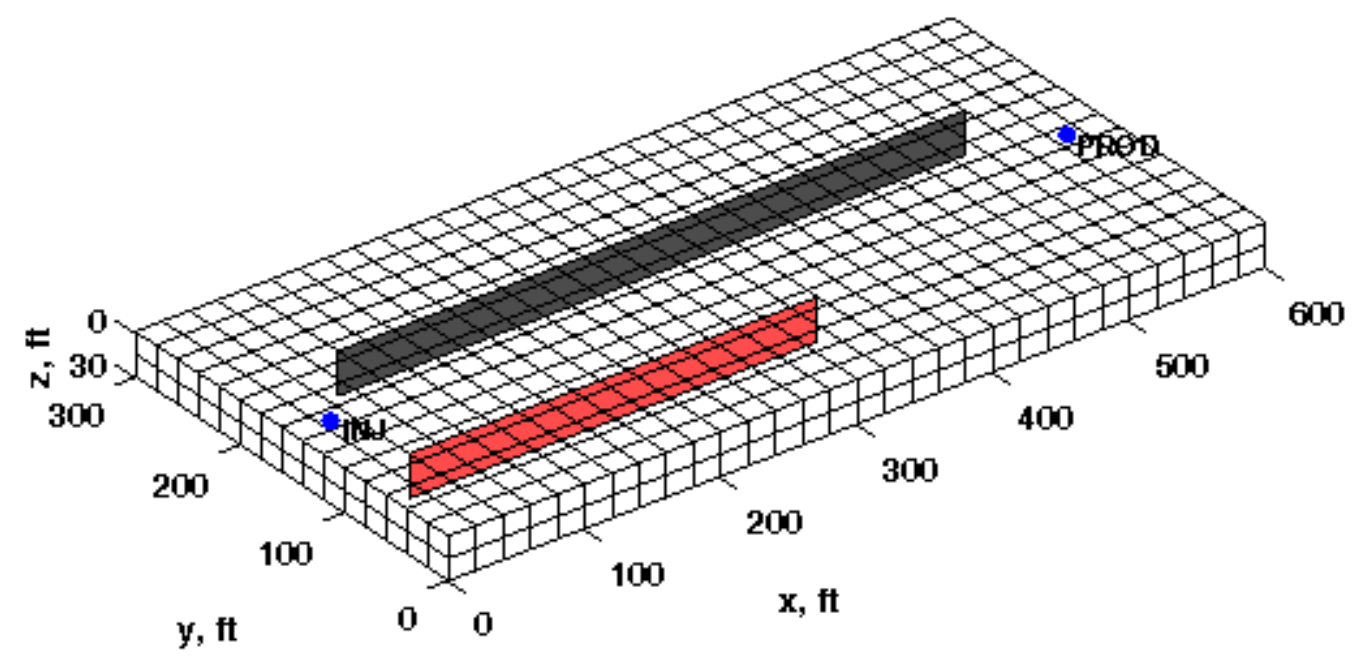

Figure 5.3: Geometrical representation of a simple 3D fractured reservoir $(600 \mathrm{ft} \times 300$ $\mathrm{ft} \times 30 \mathrm{ft}$ ) containing two isolated, discrete fractures. Both fractures are vertical and aligned with the coordinate axes.

\begin{tabular}{|c|c|c|c|}
\hline Variable & Value & Variable & Value \\
\hline Matrix porosity & 0.1 & Residual water saturation & 0.2 \\
\hline Matrix permeability & $20 \mathrm{md}$ & Residual oil saturation & 0.2 \\
\hline Fracture aperture & $0.0164 \mathrm{ft}$ & Water rel. perm. endpoint & 0.8 \\
\hline Fracture permeability & $10^{5} \mathrm{md}$ & Oil rel. perm. endpoint & 0.7 \\
\hline Init. water sat. in matrix/fracture & $0.2 / 0.0001$ & Water rel. perm. exponent & 4.0 \\
\hline Reservoir temperature & $60^{\circ} \mathrm{F}$ & Oil rel. perm. exponent & 2.0 \\
\hline \multicolumn{2}{|c|}{ Component $: \mathbf{C}_{\mathbf{1 0}} \mathbf{H}_{\mathbf{2 2}}$} \\
\hline Molecular weight (lb/lb-mol) & 142.3 & Critical temperature $\left({ }^{\circ} \mathrm{R}\right)$ & 1500.0 \\
\hline Acentric factor & 0.488 & Critical pressure $(\mathrm{psia})$ & 350.0 \\
\hline Parachor & 431.0 & Critical volume $\left(\mathrm{ft}{ }^{3} / \mathrm{lb}-\mathrm{mol}\right)$ & 10.087 \\
\hline
\end{tabular}

Table 5.2: $\quad$ Summary of properties assumed in the simulations performed for the model reservoir shown in Figure 5.3. 

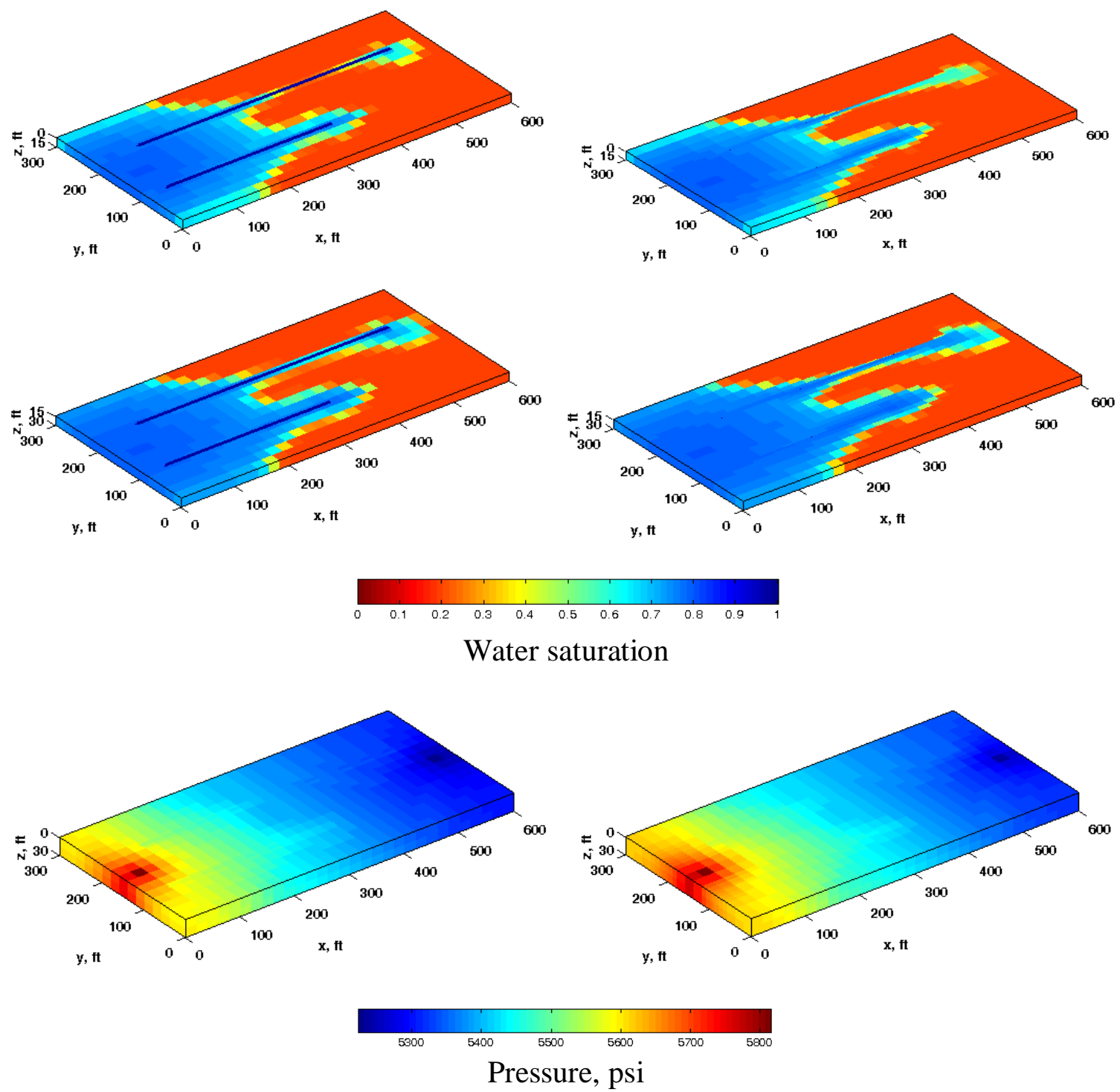

(a)

(b)

Figure 5.4: Profiles of water saturation and pressure predicted by (a) EDFM, and (b) fine-grid explicit-fracture model at $0.26 \mathrm{PV}$ injected. The first and second rows show water saturation maps in the first and second computational layers, respectively. The third row compares the pressure maps in the reservoir. 


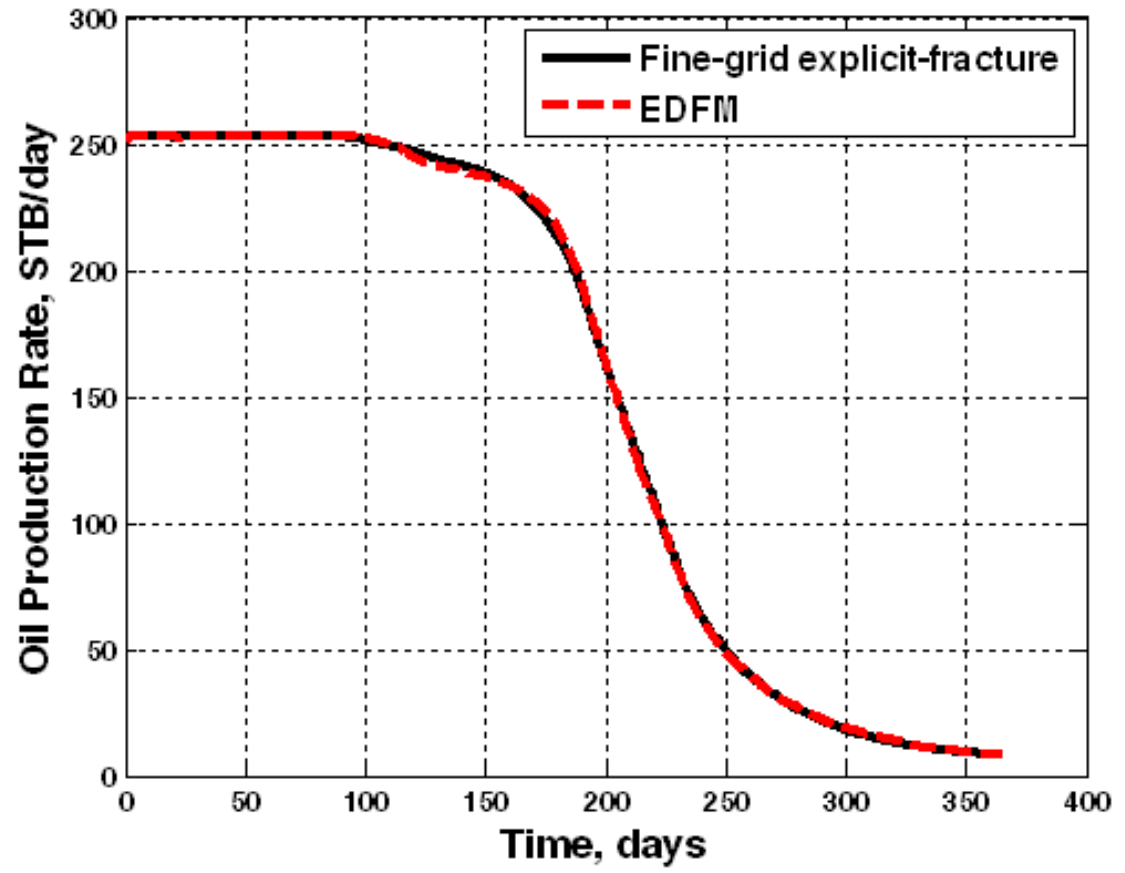

Figure 5.5: Comparison of oil production rate over a year (0.95 PV) of water injection for the model reservoir shown in Figure 5.3, calculated by the fine-grid explicit-fracture model and EDFM. 


\subsubsection{Intersected Orthogonal Fractures}

Figure 5.6 shows a 2D fractured reservoir containing three intersecting fractures. All fractures are vertical and aligned with the coordinate axes, thereby allowing us to perform standard finite-difference simulation. For the fine-grid simulation, the grid is $50 \times 55 \times 1$ cells in the $\mathrm{x}, \mathrm{y}$, and $\mathrm{z}$ directions, respectively. The cell dimensions are nonuniform in the $\mathrm{x}$ and $\mathrm{y}$ directions to accommodate refinement around fractures. The widths of the fracture gridblocks and their adjacent gridblocks were equal to the fracture aperture. Subsequently, the widths of gridblocks are increased by a factor of two, hence creating a refined grid around the fractures for up to $20 \mathrm{ft}$ on both sides of each fracture. For the EDFM simulation, a uniform $30 \times 15 \times 1$ matrix grid and 40 fracture cells are used. An injector is located in one corner of the reservoir and a producer is placed in the opposite corner.

This example is a displacement of oil by $\mathrm{CO}_{2}$, wherein the oil contains $\mathrm{CO}_{2}, \mathrm{CH}_{4}$, and $\mathrm{C}_{16} \mathrm{H}_{34}$. As described in Chapter 3, the Peng-Robinson equation-of-state (PR-EOS) is employed for the hydrocarbon phase behavior calculations. Table 5.3 describes the properties of hydrocarbon components used in these simulations. Also, the binary interaction coefficient between $\mathrm{CO}_{2}$ and hydrocarbon components is 0.12 . In this example, the initial reservoir-fluid composition is $1 \% \mathrm{CO}_{2}, 39 \% \mathrm{CH}_{4}$, and $60 \% \mathrm{C}_{16} \mathrm{H}_{34}$, while the injected-fluid composition is $98 \% \mathrm{CO}_{2}$ and $2 \% \mathrm{CH}_{4}$. The injection rate is 200 Mscf/day and both initial reservoir pressure and constant producer BHP are $1000 \mathrm{psi}$. Table 5.4 summarizes petrophysical properties used in the simulations. Matrix and fracture capillary pressures are assumed zero in the validation case studies.

The profiles of reservoir pressure, gas saturation, and $\mathrm{CO}_{2}$ mole fraction in the oleic and gaseous phases after 130 days of gas injection calculated by EDFM and finegrid explicit-fracture model are presented in Figures 5.7, 5.8, and 5.9. Again, a very good 
agreement exists between both models in all figures. Also, Figure 5.10 compares the oil and gas production rates over 600 days confirming the accuracy of the EDFM approach. The computational times for the fine-grid and EDFM simulations were 25.6 hours and 24.6 minutes, respectively.

The effect of phase behavior on fluid saturations is very pronounced in this example. Figure 5.11 shows the profiles of gas saturation simulated by EDFM after one month, three months, six months, and one year of gas injection. At the initial conditions as described in Table 5.4, both oil and gas phases are present in the reservoir. In a portion of the reservoir away from the wells and fractures and consequently from the injected fluid, a single-phase oil is formed owing to the pressure increase as time progresses (excluding irreducible water in the reservoir). However, in areas close to the wells and fractures, gas saturation gradually increases as $\mathrm{CO}_{2}$ overall mole fraction increases.

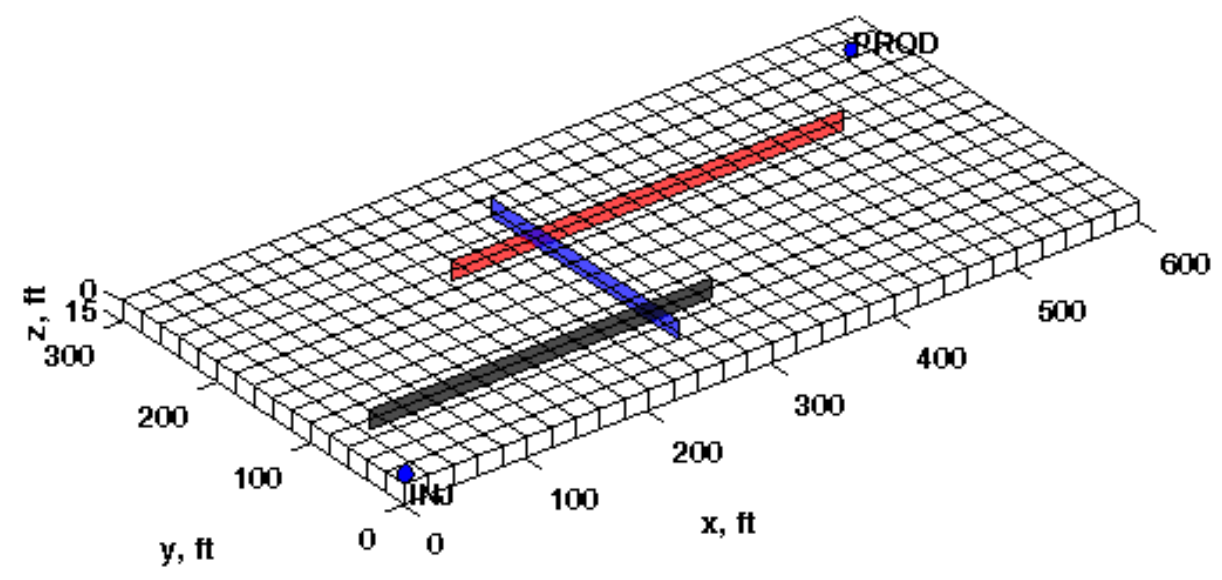

Figure 5.6: Geometrical representation of a simple fractured reservoir $(600 \mathrm{ft} \times 300 \mathrm{ft} \times$ $15 \mathrm{ft})$ containing three intersected fractures. All fractures are vertical and aligned with the coordinate axes. 


\begin{tabular}{|c|c|c|c|}
\hline Component & $\mathbf{C H}_{\mathbf{4}}$ & $\mathbf{C}_{\mathbf{1 6}} \mathbf{H}_{\mathbf{3 4}}$ & $\mathbf{C O}_{\mathbf{2}}$ \\
\hline Molecular weight $(\mathrm{lb} / \mathrm{lb}-\mathrm{mol})$ & 16.0 & 222.0 & 44.0 \\
\hline Critical temperature $\left({ }^{\circ} \mathrm{R}\right)$ & 343.1 & 1322.4 & 547.6 \\
\hline Critical pressure $(\mathrm{psia})$ & 667.2 & 252.1 & 1071.6 \\
\hline Critical volume $\left(\mathrm{ft}^{3} / \mathrm{lb}-\mathrm{mol}\right)$ & 1.586 & 13.377 & 1.506 \\
\hline Acentric factor & 0.008 & 0.684 & 0.225 \\
\hline Parachor & 71.0 & 831.9 & 49.0 \\
\hline
\end{tabular}

Table 5.3: Properties of the hydrocarbon components used in the simulations performed for the model reservoir shown in Figure 5.6.

\begin{tabular}{|c|c|c|c|}
\hline Variable & Value & Variable & Value \\
\hline Matrix porosity & 0.1 & Residual water saturation & 0.1 \\
\hline Matrix permeability & $1 \mathrm{md}$ & Residual oil saturation & 0.1 \\
\hline Fracture aperture & $0.0264 \mathrm{ft}$ & Residual gas saturation & 0.0 \\
\hline Fracture permeability & $8 \times 10^{4} \mathrm{md}$ & Water rel. perm. endpoint & 0.4 \\
\hline Initial water saturation & 0.1 & Oil rel. perm. endpoint & 0.6 \\
\hline Initial oil saturation & 0.633 & Gas rel. perm. endpoint & 0.6 \\
\hline Initial gas saturation & 0.267 & Water rel. perm. exponent & 3.0 \\
\hline Reservoir temperature & $160^{\circ} \mathrm{F}$ & Oil rel. perm. exponent & 2.0 \\
\hline Reservoir pressure & $1000 \mathrm{psi}$ & Gas rel. perm. exponent & 2.0 \\
\hline
\end{tabular}

Table 5.4: Summary of properties used in the simulations performed for the model reservoir shown in Figure 5.6. 


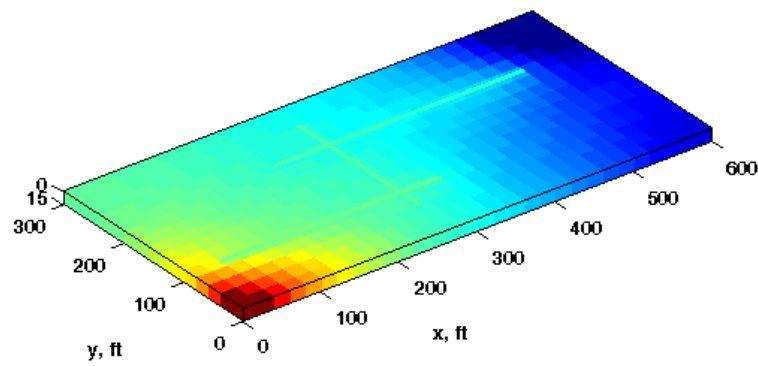

(a)

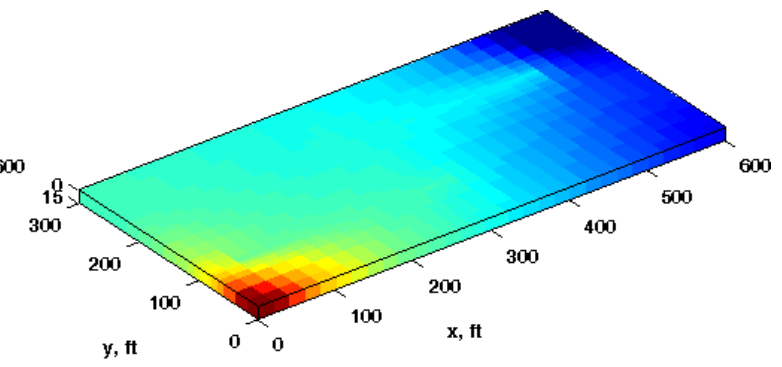

(b)

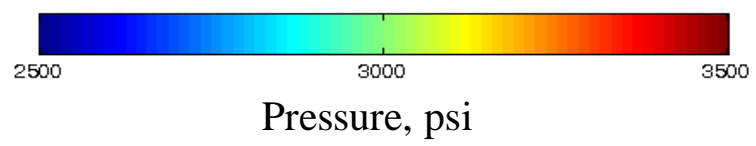

Figure 5.7: Profiles of reservoir pressure predicted by (a) EDFM and (b) fine-grid explicit-fracture model after 130 days of gas injection for the model reservoir shown in Figure 5.6.

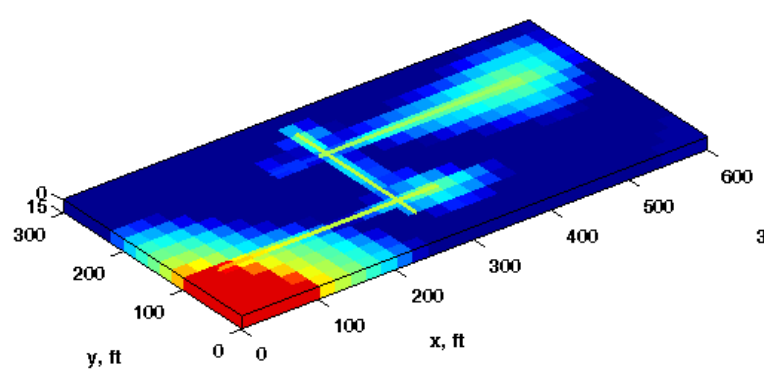

(a)

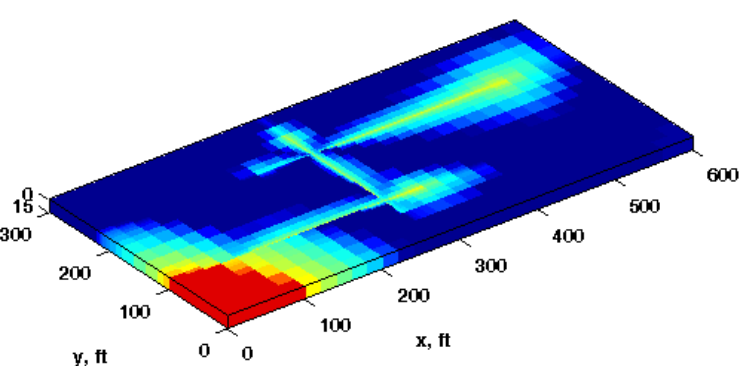

(b)

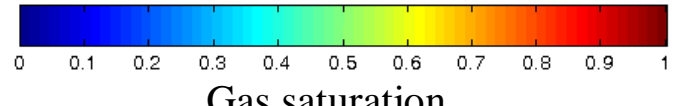

Gas saturation

Figure 5.8: Profiles of gas saturation predicted by (a) EDFM and (b) fine-grid explicitfracture model after 130 days of gas injection for the model reservoir shown in Figure 5.6. 

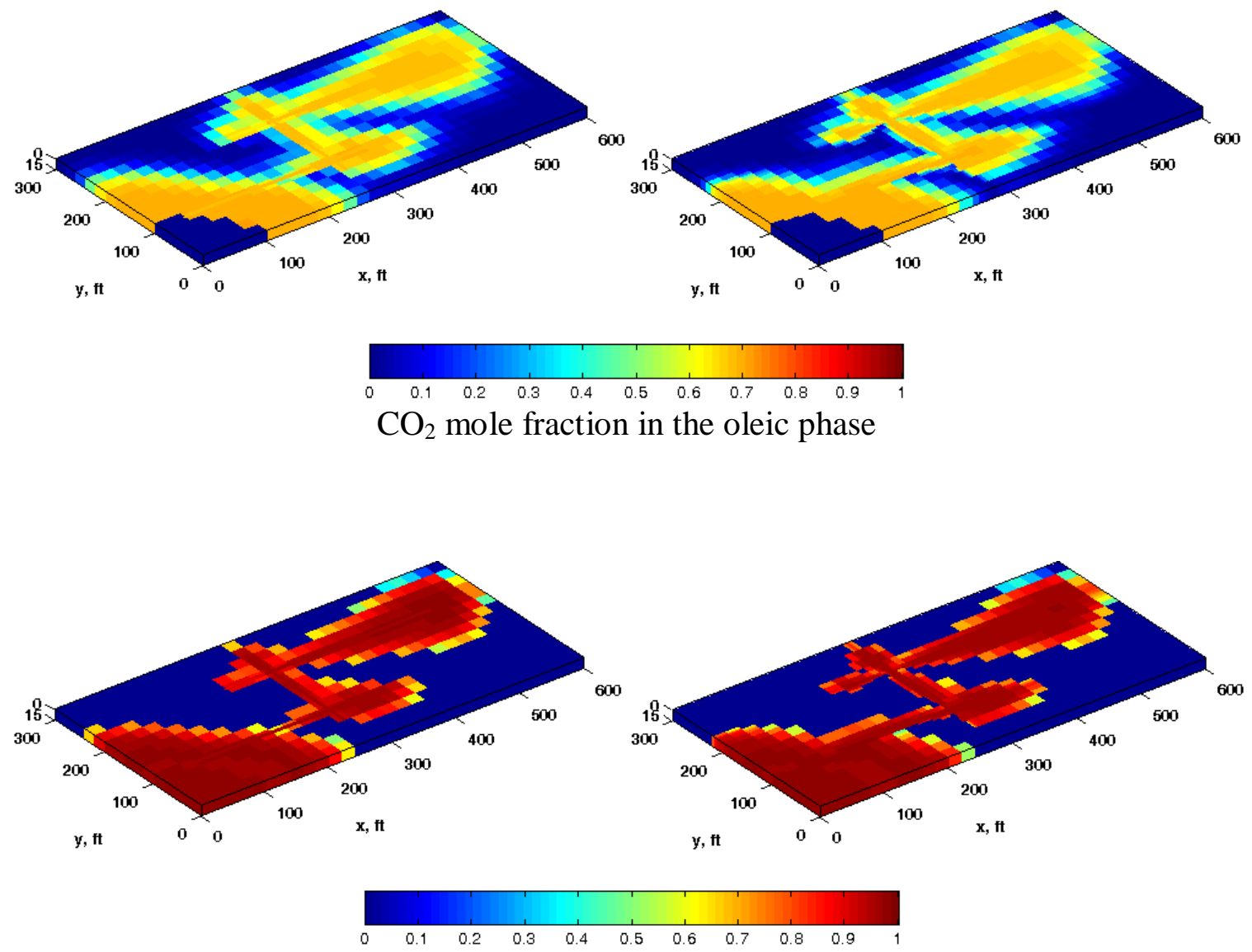

$\mathrm{CO}_{2}$ mole fraction in the gaseous phase

(a)

(b)

Figure 5.9: Profiles of $\mathrm{CO}_{2}$ mole fraction in the oleic and gaseous phases predicted by (a) EDFM and (b) fine-grid explicit-fracture model after 130 days of gas injection for the model reservoir shown in Figure 5.6. 


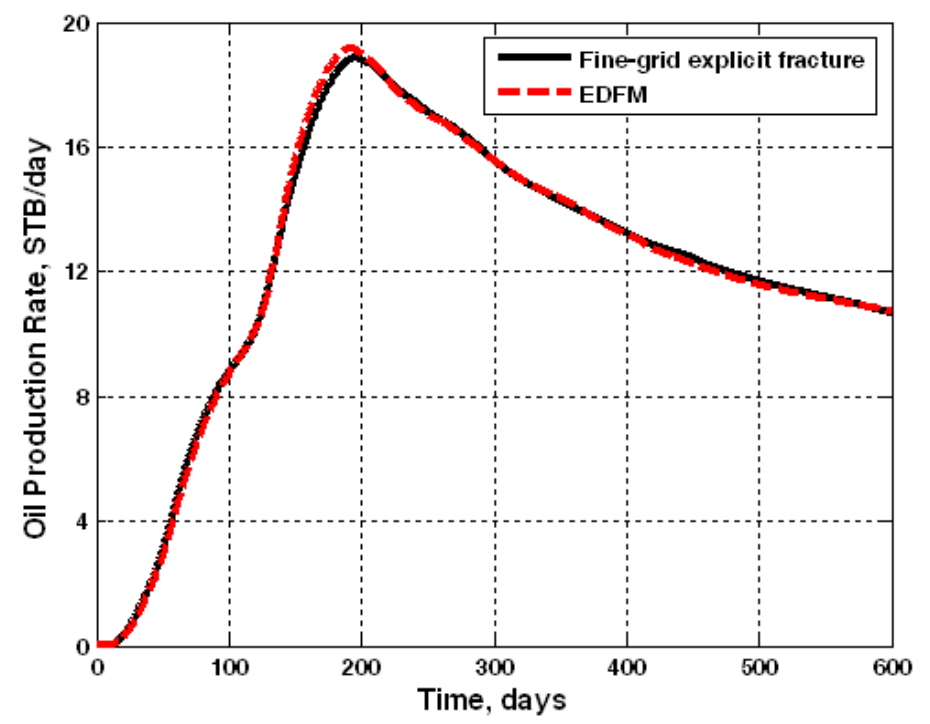

(a)

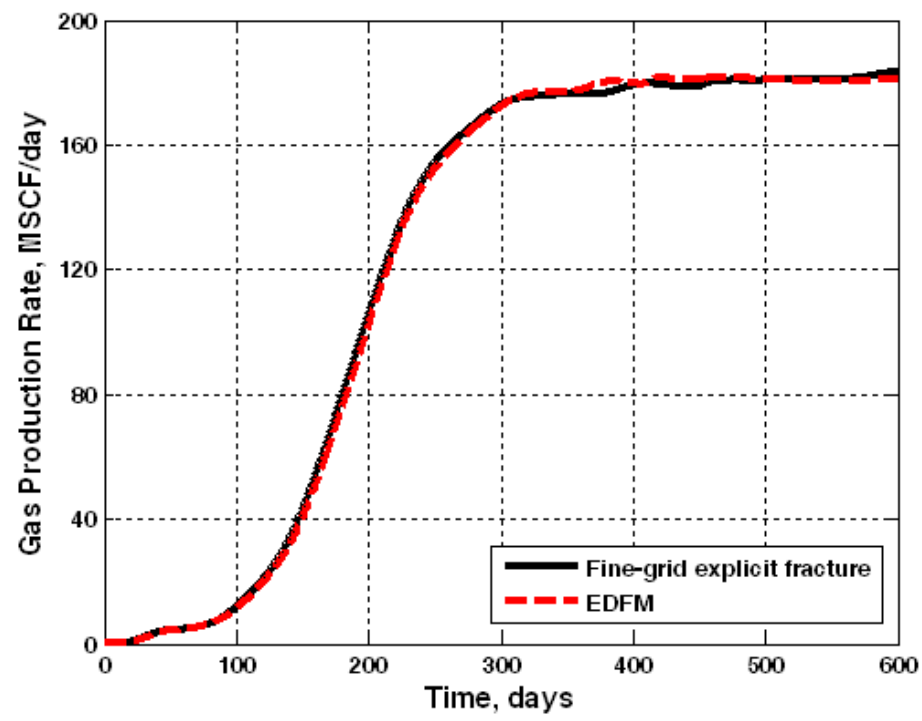

(b)

Figure 5.10: Comparison of (a) oil production rate, and (b) gas production rate over 600 days of gas injection for the model reservoir shown in Figure 5.6, calculated by the fine-grid explicit-fracture model and EDFM. 


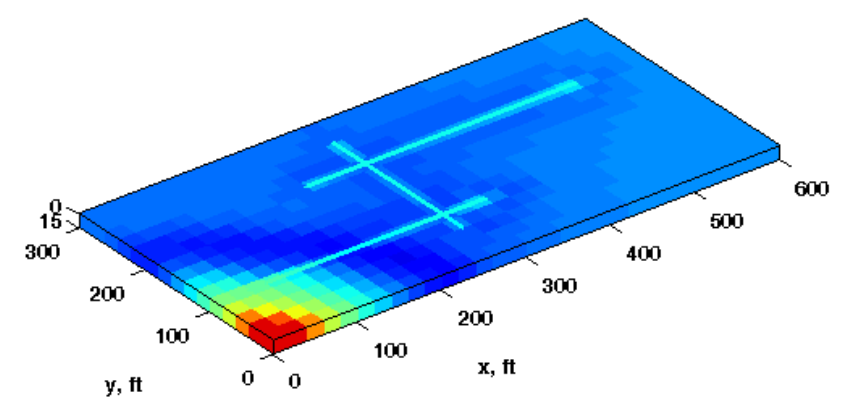

(a)

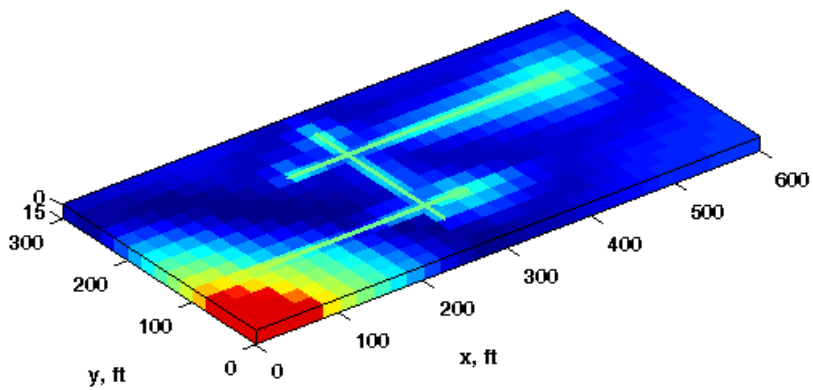

(b)

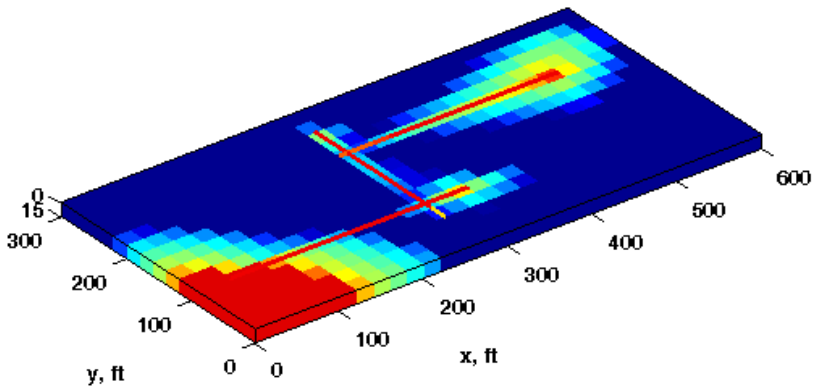

(c)

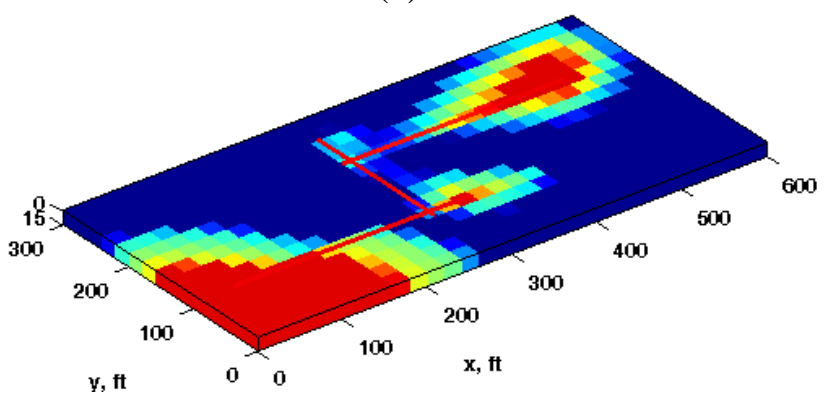

(d)

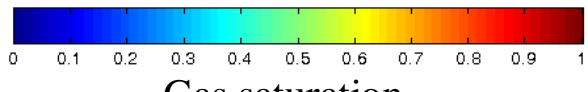

Gas saturation

Figure 5.11: Profiles of gas saturation predicted by EDFM after (a) 1 month, (b) 3 months, (c) 6 months, and (d) 1 year of gas injection. 


\subsubsection{Non-Orthogonal Non-Aligned Fracture}

The next validation case comprises a non-aligned fracture to investigate the accuracy of the EDFM approach for modeling arbitrary-oriented fractures. In this example, we compare the results of the EDFM approach to the results of a Cartesian single-porosity model that uses a very fine stair-stepping representation of the nonaligned fracture, as shown in Figure 5.12. The domain dimensions are $4 \times 4 \times 1 \mathrm{ft}$. The fracture endpoints are located at $(0.56,0.41)$ and $(3.49,3.34)$. An injector is located at one corner of the domain, injecting water at $0.001 \mathrm{bbl} / \mathrm{day}$, and a producer is placed at the opposite corner. Initial domain pressure and the constant producer BHP are 3000 psi. Likewise, $\mathrm{C}_{10} \mathrm{H}_{22}$ is the only hydrocarbon component in these simulations. All other properties used in the simulations, except the fracture permeability, are summarized in Table 5.5.

\begin{tabular}{|c|c|c|c|}
\hline Variable & Value & Variable & Value \\
\hline Matrix porosity & 0.2 & Residual water saturation & 0.2 \\
\hline Matrix permeability & $1 \mathrm{md}$ & Residual oil saturation & 0.2 \\
\hline Fracture aperture & $0.05 \mathrm{ft}$ & Water rel. perm. endpoint & 0.8 \\
\hline Init. water sat. in matrix & 0.2 & Oil rel. perm. endpoint & 0.7 \\
\hline Init. water sat. in fracture & 0.0001 & Water rel. perm. exponent & 4.0 \\
\hline Domain temperature & $60^{\circ} \mathrm{F}$ & Oil rel. perm. exponent & 2.0 \\
\hline \multicolumn{5}{|c|}{ Component $: \mathbf{C}_{\mathbf{1 0}} \mathbf{H}_{\mathbf{2 2}}$} \\
\hline Molecular weight (lb/lb-mol) & 142.3 & Critical temperature $\left({ }^{\circ} \mathrm{R}\right)$ & 1500.0 \\
\hline Acentric factor & 0.488 & Critical pressure $(\mathrm{psia})$ & 350.0 \\
\hline Parachor & 431.0 & Critical volume $\left(\mathrm{ft}{ }^{3} / \mathrm{lb}-\mathrm{mol}\right)$ & 10.087 \\
\hline \multicolumn{5}{|c|}{ Description of Fine-Grid } \\
\hline$\Delta \mathrm{x}, \mathrm{ft}$ & $0.2,3 \times 0.05,132 \times 0.025,3 \times 0.05,0.2$ & $\Delta \mathrm{y}, \mathrm{ft}$ & $0.2,3 \times 0.05,132 \times 0.025,3 \times 0.05,0.2$ \\
\hline
\end{tabular}

Table 5.5: Summary of properties used in the simulations performed for the model reservoir shown in Figure 5.12. 


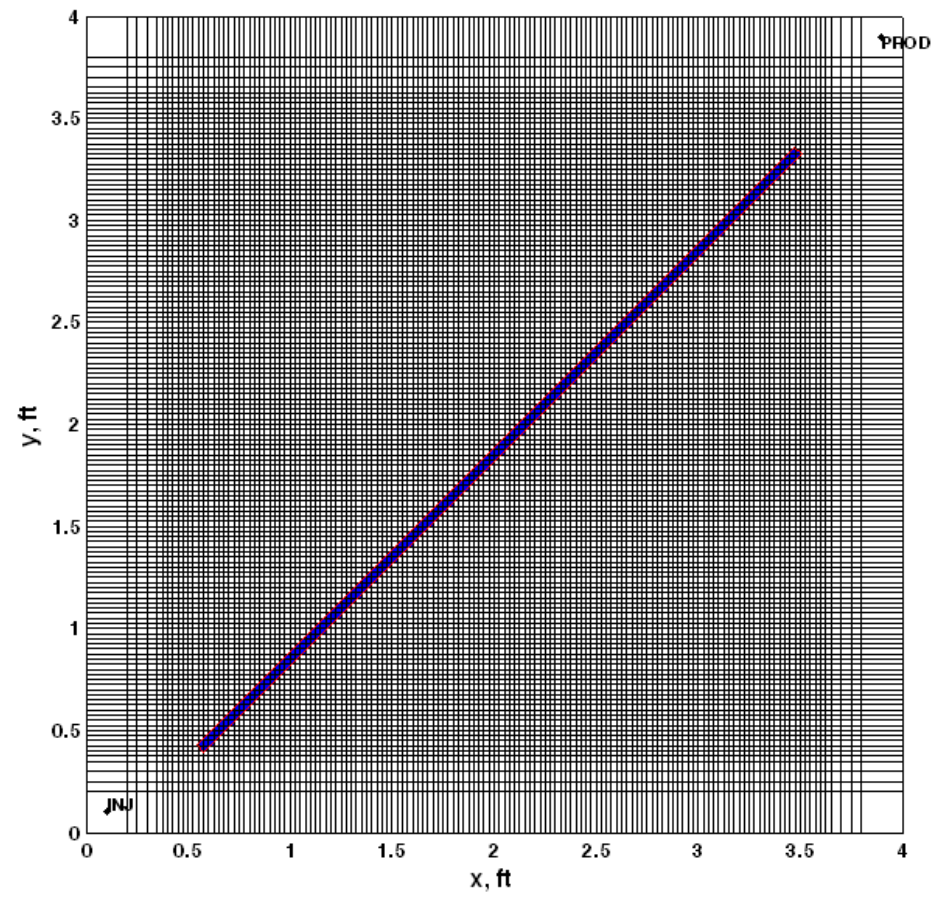

(a)

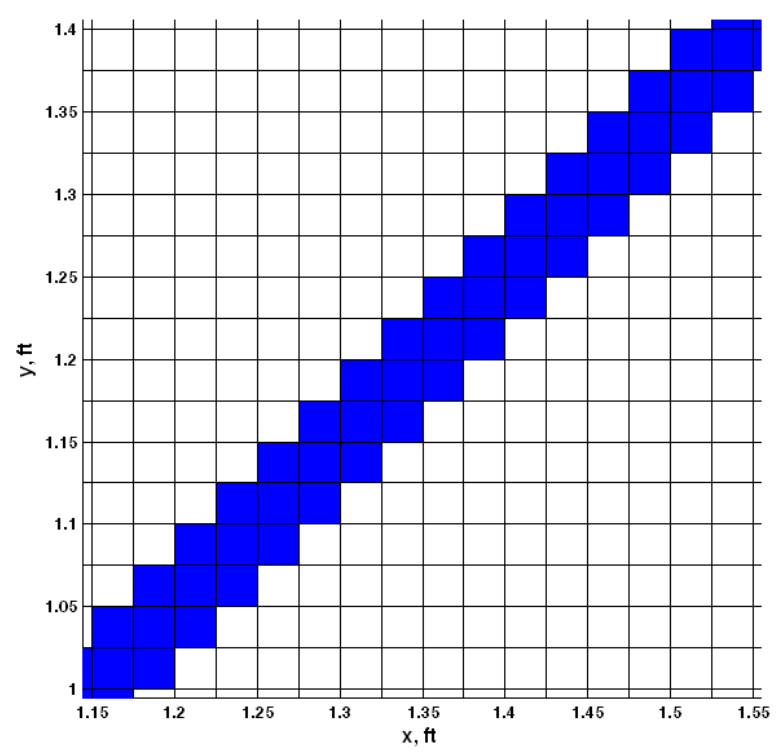

(b)

Figure 5.12: (a) A very fine stair-stepping representation of a non-aligned fracture used to investigate the accuracy of the EDFM approach for modeling arbitraryoriented fractures. The domain dimensions are $4 \times 4 \times 1 \mathrm{ft}$. (b) An enlarged region of the model shown in Figure 5.12a, depicting the size of the gridblocks used to represent the fracture gridblocks. 
We assume straight-line relative permeability curves (with zero residual saturations and endpoints equal to one) for the fracture in the simulations. For the finegrid explicit-fracture simulation, the grid is $140 \times 140 \times 1$ cells in the $\mathrm{x}, \mathrm{y}$, and $\mathrm{z}$ directions, respectively, and the cell dimensions are non-uniform in both $\mathrm{x}$ and $\mathrm{y}$ directions, as depicted in Figure 5.12a and described in Table 5.5.

The permeability of fracture cells in the fine-grid simulation using stair-stepping approach is $100 \mathrm{md}$. In order to mimic the simulation of fluid flow for the fine-grid simulation using the EDFM method, we adjusted the permeability of the $45^{\circ}$-deviated fracture to $70.7 \mathrm{md}(100 / \sqrt{2})$ in the EDFM approach to model the same fluid-flow behavior as for the stair-stepping case. The reason for modification of the permeability using $\sqrt{2}$ multiplier is given below:

As depicted in Figure 5.13, for the EDFM approach, flow between points 1 and 2 occurs only in one direction (parallel to fracture) while for the fine-grid simulation flow occurs in a stair-step manner resulting from the sum of the two routes shown in the figure. Owing to the location of wells and orientation of the fracture in Figure 5.12a, we assume that the flow rates in the two routes are equal. Hence,

$$
\begin{aligned}
& q_{E D F M}=q_{1, \text { fine }}+q_{2, \text { fine }}=2 q_{1, \text { fine }}, \\
& \frac{k_{f, E D F M} \omega_{f, E D F M} h_{f}}{\mu} \frac{\Delta P}{\sqrt{2} \Delta x_{f i n e}}=\frac{2 k_{f, f i n e} \Delta x_{f i n e} h_{f}}{\mu} \frac{\Delta P}{2 \Delta x_{f i n e}}, \\
& \frac{k_{f, E D F M} \omega_{f, E D F M}}{\sqrt{2}}=k_{f, \text { fine }} \Delta x_{f i n e}, \\
& \frac{k_{f, E D F M} \times 0.05}{\sqrt{2}}=k_{f, \text { fine }} \times 0.025, \\
& k_{f, \text { fine }}=\sqrt{2} k_{f, E D F M} .
\end{aligned}
$$


In order to perform similar fluid-flow simulations using two approaches (EDFM and fine-grid), as can be seen above, mimicking non-orthogonal fractures in Cartesian coordinates using a fine-grid is not an easy task for a general case. For different cases the above computations have to be carried out in order to come up with a corresponding permeability for the fine-grid simulations.

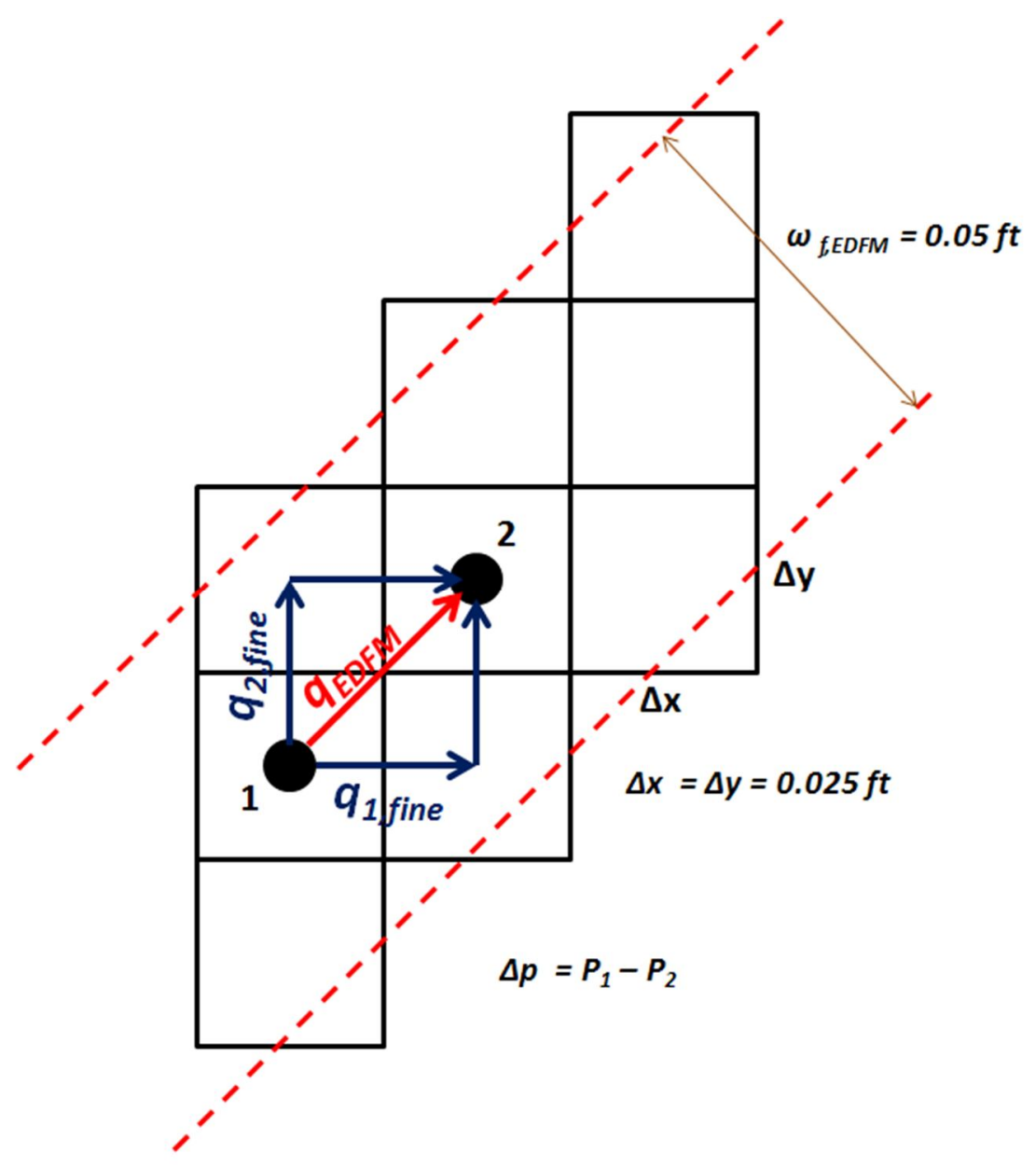

Figure 5.13: Representation of fracture permeability in the fine-grid simulation with respect to the fracture permeability in the EDFM simulations. 
We examine three EDFM simulations with different grid resolutions to check the method for convergence as the grid is refined. The first, second, and third simulations use uniform $10 \times 10 \times 1,20 \times 20 \times 1$, and $40 \times 40 \times 1$ matrix grids, respectively. Figure 5.14 presents water saturation profiles after 140 days of water injection ( $0.233 \mathrm{PV}$ injected) calculated by fine-grid and EDFM simulations. Also, Figure 5.15 compares the oil production rate over 350 days of water injection. From both figures, it is inferred that the EDFM approach could accurately reproduce the results of the fine-grid simulation using moderate grid refinement, demonstrating the general level of accuracy of the EDFM approach for modeling arbitrary-oriented fractures.

We performed the fine-grid simulation using the parallel processing option of GPAS with four processors and the computational time was 88.3 hours. Our main goal for performing this study was to compare our results with that of a very fine-grid simulation. We note here that our goal was not to find the minimum number of gridblocks required for the fine-grid simulation to obtain a converged solution. The computational times for three EDFM simulations on a single processor were 2, 13, and 127 seconds for various refinements as stated above.

Owing to excessive computational efforts to carry out detailed fine-grid simulation, we did not perform further studies to compare the EDFM approach with finegrid simulations for domains with multiple intersecting fractures. However, the presented example shows good level of agreement for a single non-orthogonal fracture. 


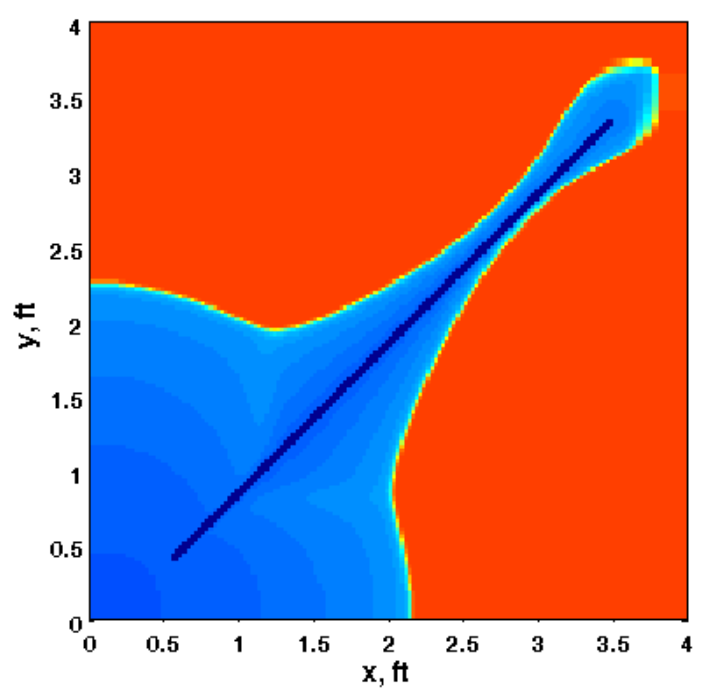

(a)

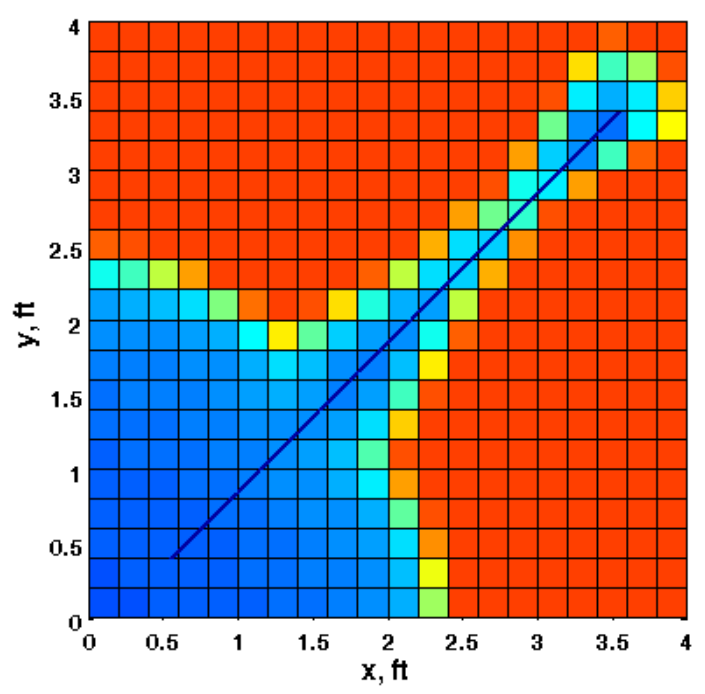

(c)

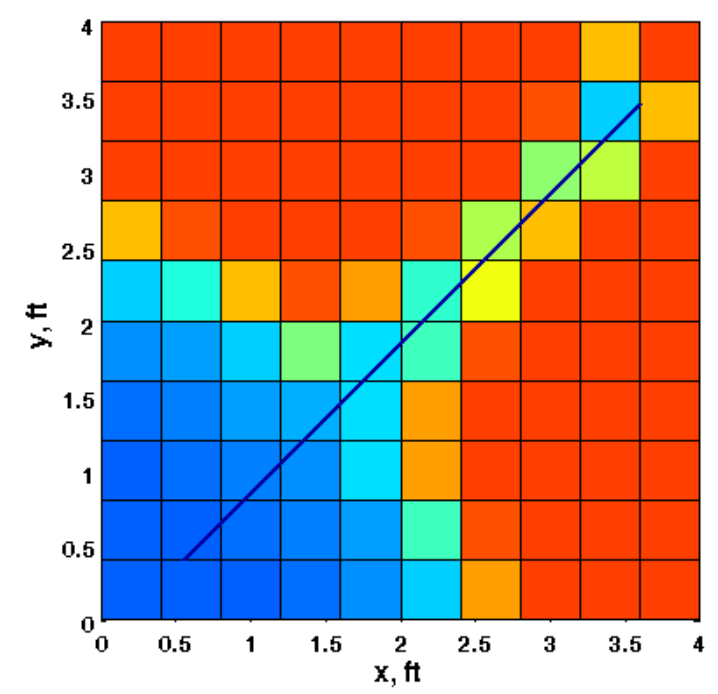

(b)

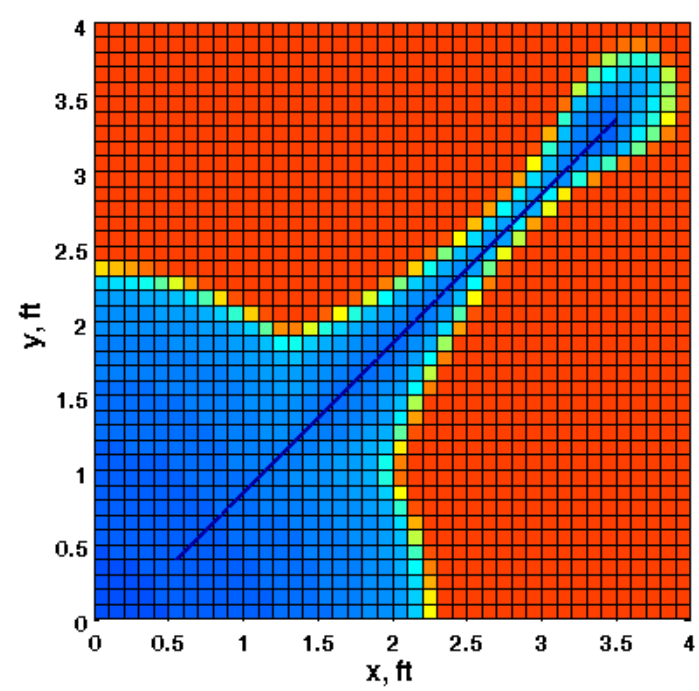

(d)

Figure 5.14: Profiles of water saturation predicted by (a) fine-grid explicit-fracture model, (b) EDFM approach using a $10 \times 10 \times 1$ matrix grid, (c) EDFM approach using a $20 \times 20 \times 1$ matrix grid, and (d) EDFM approach using a $40 \times 40 \times 1$ matrix grid after 140 days of water injection ( $0.233 \mathrm{PV}$ injected). 


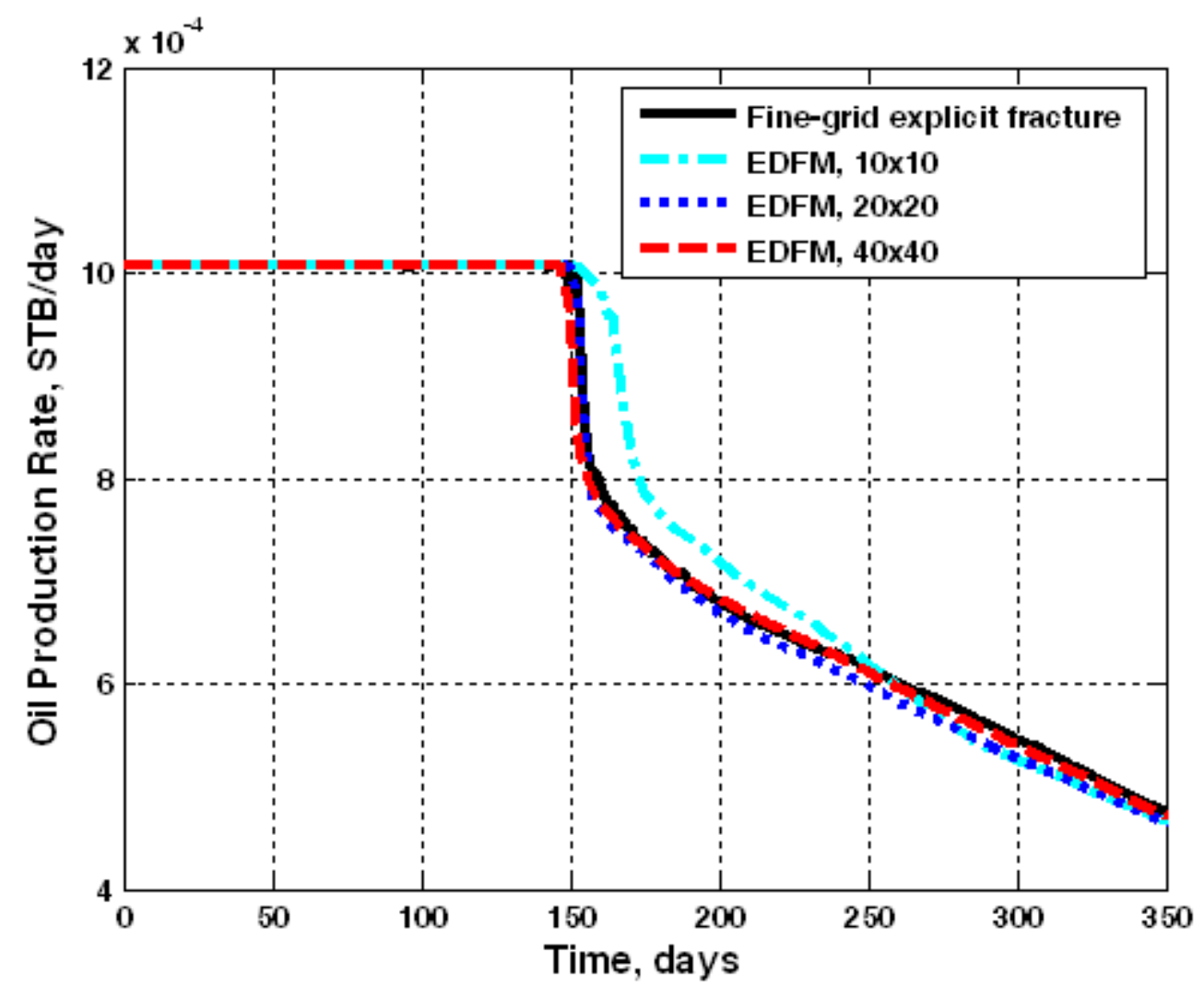

Figure 5.15: Comparison of oil production rate over 350 days of water injection for the model reservoir shown in Figure 5.12a, calculated by the fine-grid and EDFM simulations. 


\subsection{Highlights OF THE CHAPTer}

- The accuracy of the EDFM approach for modeling primary production from multiply-fractured horizontal wells is confirmed by its match with the analytical solutions for two production schemes of constant-pressure production and constant-rate production.

- The accuracy of the EDFM approach is confirmed by comparing the EDFM results with the fine-grid, explicit-fracture simulations for case studies including isolated fractures, intersected orthogonal fractures, and a nonorthogonal non-aligned fracture. The case studies examined both waterflooding and compositional gas injection.

- Comparison of our results using the EDFM approach with fine-grid simulations shows that accurate results can be achieved with moderate grid refinements. Hence, EDFM offers a computationally-efficient approach for simulating fluid flow in fractured reservoirs. 


\section{Chapter 6: Case Studies using EDFM}

In the first part of this chapter, we present examples of water-flooding, gas injection with a cubic equation-of-state, and primary depletion to demonstrate the performance and applicability of the developed approach for simulating fluid flow in naturally fractured reservoirs. GPAS is used for all examples presented in the first part. In the first example, we investigate the effect of capillary pressure on water imbibition from fractures to matrix. Moreover, we perform a grid sensitivity study to check the

convergence of the method as the grid is refined. The second example presents a compositional gas injection displacement to show the effect of gas-oil gravity drainage on oil recovery. In the third case study, we examine water-flooding into a 3D model and investigate the effect of fracture inclination. The last example examines primary depletion of a synthetic 3D reservoir to show the applicability of the EDFM approach for modeling a large number of obliquely dipping fractures.

The second part of this chapter is a part of a study performed during a summer internship with Chevron Energy Technology Company. As described in the previous chapters, Li and Lee (2008) developed the EDFM approach for simulating fluid flow in vertical fractures. They implemented the method for a Chevron proprietary reservoir simulator, called CHEARS. During this internship, we compared the EDFM approach to an unstructured discrete fracture model (called USDFM) and a dual permeability method for simulating multiphase flow in NFRs. We gratefully thank Chevron Energy Technology Company for the financial support of this work and for permitting its publication. 


\subsection{Simulation EXAMPLES USING EDFM IN GPAS}

In this section, we present various simulation case studies to show the applicability, robustness, and performance of the EDFM approach for simulating fluid flow in naturally fractured reservoirs.

\subsubsection{D Water-Flooding (Effect of Capillary Pressure)}

The purpose of the first example is to show the applicability of the EDFM approach for modeling a well-known recovery mechanism in naturally fractured reservoirs. Water-flooding produces oil from NFRs through spontaneous imbibition of water from fractures into the rock matrix and expulsion of oil from the rock matrix to the fractures and production wells. However, this capillary driving force is strong only when large capillary pressure contrasts exist between matrix and fractures, and when the rock matrix is water-wet. Many naturally fractured reservoirs are oil-wet or mixed-wet formations, leading to poor performance of water-flooding in these reservoirs. Here, we present two water-flooding simulations performed in the absence and presence of capillary pressure in the matrix.

The model reservoir evaluated in this example is a synthetic 2D reservoir shown in Figure 6.1. The model contains 14 long, vertical, natural fractures striking at different orientations. The synthetic model is a quarter of a five-spot well pattern where an injector is located in one corner and a producer is installed in the opposite corner. The aperture and permeability of all fractures are $0.025 \mathrm{ft}$ and $7 \times 10^{5} \mathrm{md}$, respectively. Also, porosity and permeability of matrix are 0.1 and $20 \mathrm{md}$, respectively. All other petrophysical properties are similar to those reported in Table 5.2, and straight-line relative permeability is assumed for the fractures. Initial reservoir pressure and the constant producer BHP are $3000 \mathrm{psi}$. The water injection rate is $100 \mathrm{bbl} / \mathrm{day}$. The matrix grid is 
$20 \times 20 \times 1$, where the sizes of each gridblock in the $\mathrm{x}, \mathrm{y}$, and $\mathrm{z}$ directions are 25,25 , and 20 $\mathrm{ft}$, respectively. Also, fractures are discretized into 156 control volumes in the fracture domain. Both matrix and fracture capillary pressures are assumed zero in the first simulation, but we use the following relation for matrix capillary pressure in the second simulation:

$$
P_{\text {cow }}=-5 \times \ln \left(S_{w}\right) \text {, }
$$

where $S_{w}$ is the water saturation and $P_{\text {cow }}$ is the oil-water capillary pressure in psi. Fracture capillary pressure is zero in the second simulation. We used the above matrix capillary pressure relation since this was the relation used by Monteagudo and Firoozabadi (2004). The capillary pressure equation presented by Brooks and Corey (1964) would have also been an alternative equation to be used in this example.

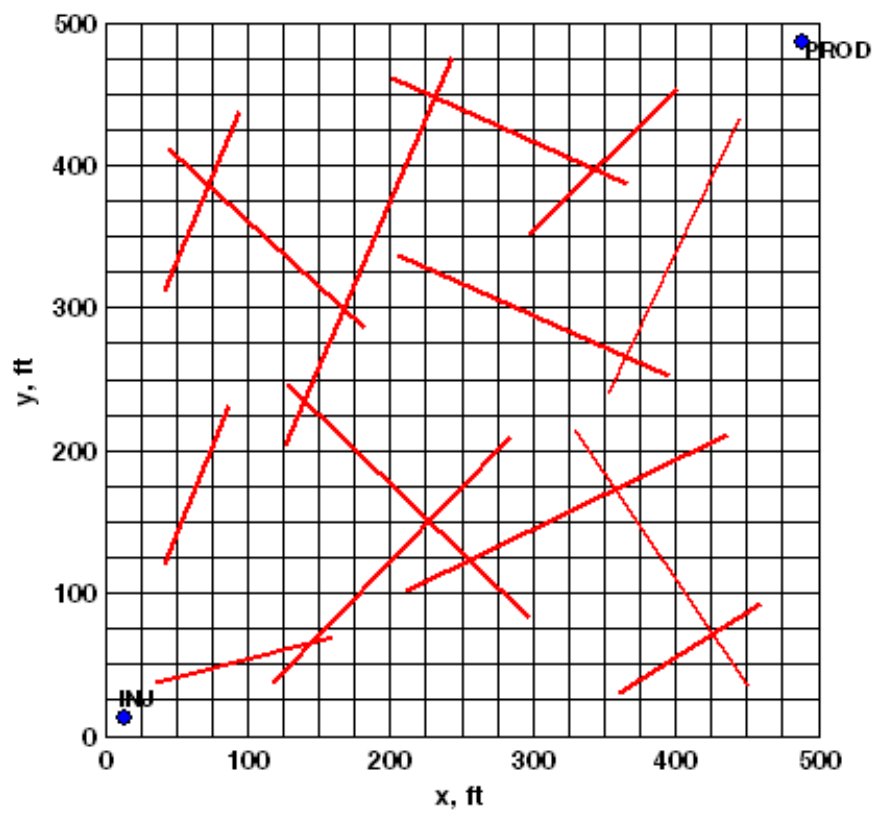

Figure 6.1: A synthetic 2D fractured reservoir $(500 \mathrm{ft} \times 500 \mathrm{ft} \times 20 \mathrm{ft})$ considered in Example 1. The model contains 14 long, vertical fractures striking at different orientations. 
Figure 6.2 compares the oil production rate calculated by EDFM for both simulations, while Figure 6.3 depicts the water saturation profiles at 0.17 and 0.28 of pore volumes injected. The first row in Figure 6.3 corresponds to the first case and the second row corresponds to the case wherein capillary contrasts exist between matrix and fractures. Water channeling through the highly conductive fracture network is evident in Figure 6.3. Both Figures 6.2 and 6.3 indicate that the water breakthrough is significantly delayed when there is a difference in the capillary pressures of matrix and fracture, owing to an improved sweep. As shown in Figure 6.3, in the presence of capillary contrasts, invasion of water is more apparent in the matrix around fractures as a result of stronger spontaneous imbibition.

The oil recovery factors after one year of water injection are 0.441 and 0.474 in the absence and presence of capillary pressure contrasts, respectively. Consequently, favorable rock wettability along with capillary contrasts between matrix and fracture resulted in $7.5 \%$ increased oil recovery in this example. The consistency of our results with well-established findings of previous studies demonstrates the applicability of the EDFM approach for modeling different recovery mechanisms. The computational times in the absence and presence of capillary pressure contrasts were 2.5 and 3.8 minutes, respectively.

To evaluate the impact of grid resolution on accuracy of the EDFM approach, we also performed a mesh sensitivity study for the case in the absence of capillary pressure contrasts. Retaining the same fracture network, we examined three additional EDFM simulations that use uniform $40 \times 40 \times 1,50 \times 50 \times 1$, and $80 \times 80 \times 1$ matrix grids, respectively, to check the convergence of the method as the grid is refined. Figure 6.4 compares the oil production rate simulated by four grid resolutions under consideration. Also, Figure 6.5 shows the water saturation profiles after 250 days of water injection ( $0.28 \mathrm{PV}$ injected) as 
we refined the grid. As it can be seen, the results which correspond to the $40 \times 40 \times 1$ mesh are close to the results generated using $50 \times 50 \times 1$ and $80 \times 80 \times 1$ matrix grids. Figures 6.4 and 6.5, once again, verify that the EDFM approach can achieve high accuracy using moderate mesh refinement. The computational times for three additional EDFM simulations were 26, 63, and 386 minutes for various refinements as stated above.

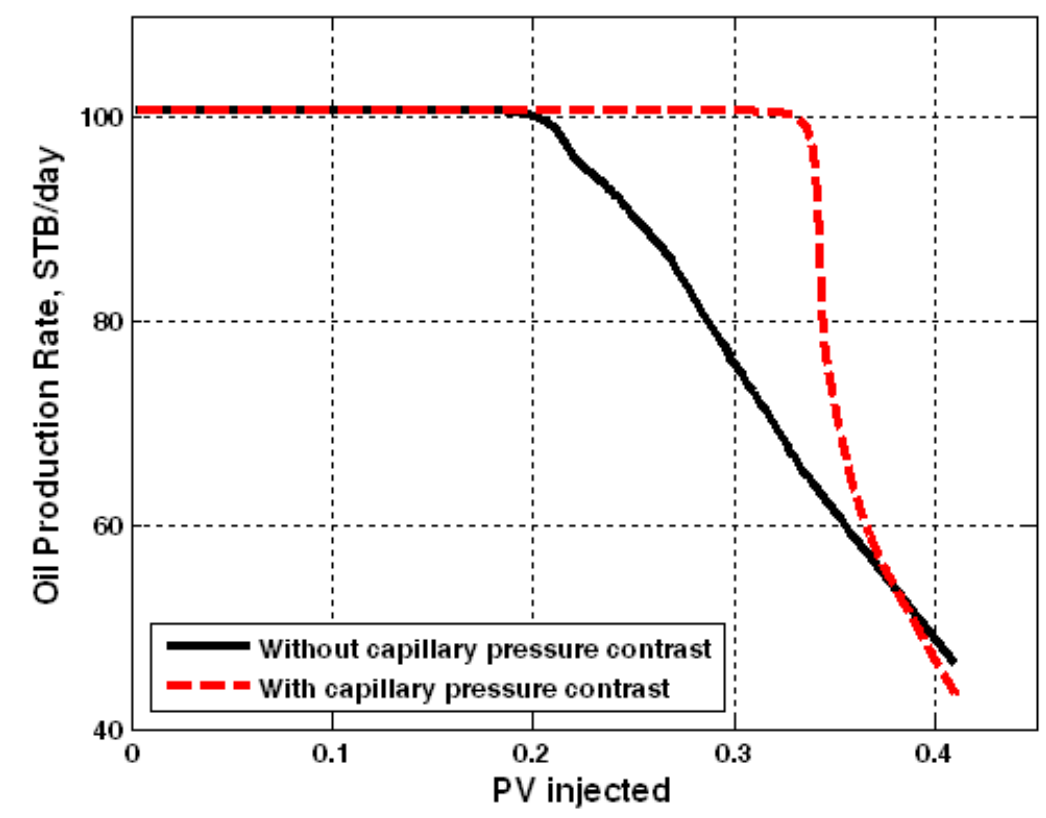

Figure 6.2: Comparison of oil production rate calculated in the absence and presence of capillary pressure contrasts between matrix and fracture for the model reservoir shown in Figure 6.1. 

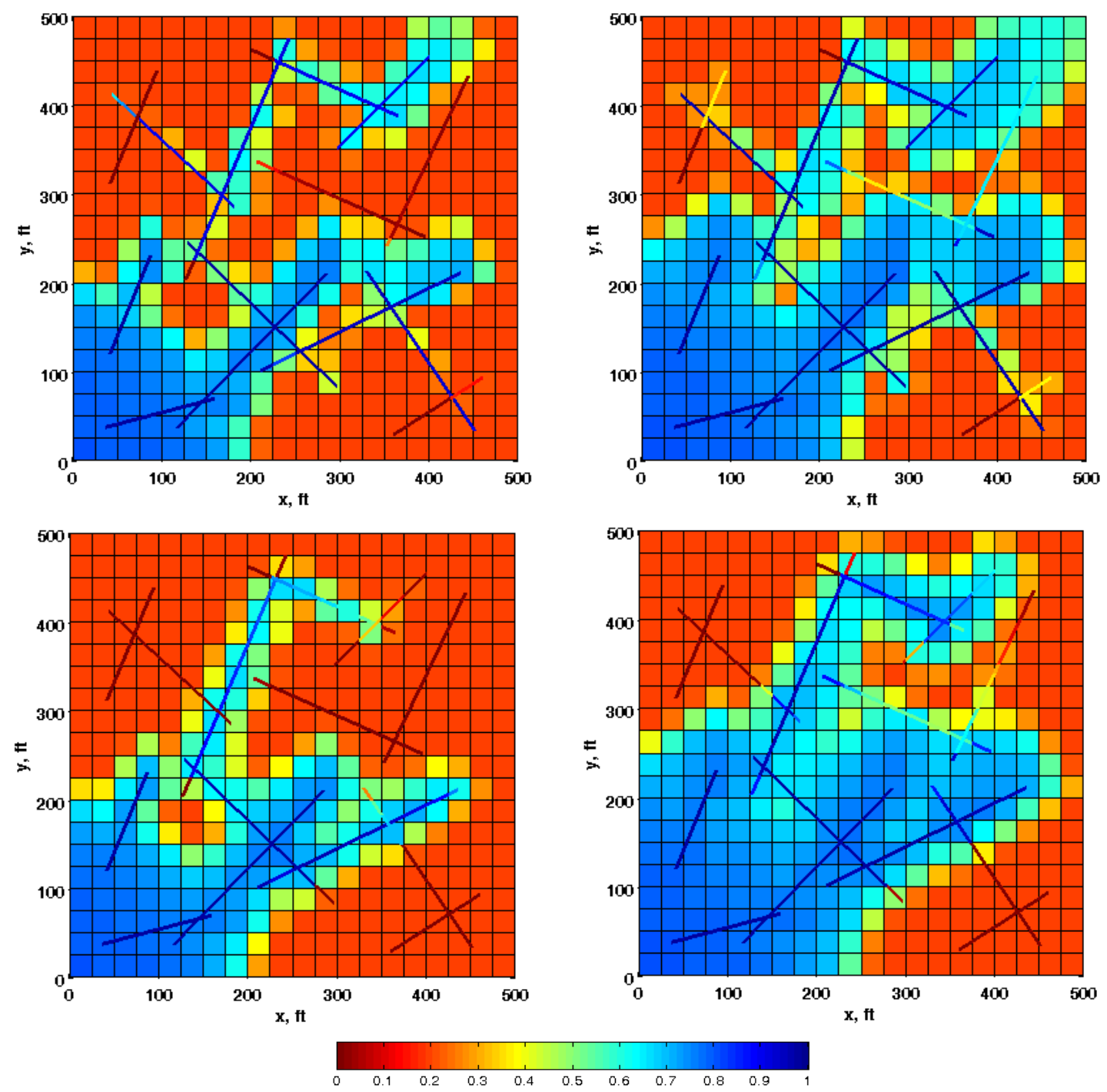

Water saturation

(a)

(b)

Figure 6.3: Water saturation profiles calculated by EDFM at (a) $0.17 \mathrm{PV}$ injected (150 days), and (b) $0.28 \mathrm{PV}$ injected (250 days). The first row shows water saturation maps simulated in the absence of capillary contrasts and the second row shows water saturation maps simulated in the presence of capillary contrasts between fractures and matrix. 


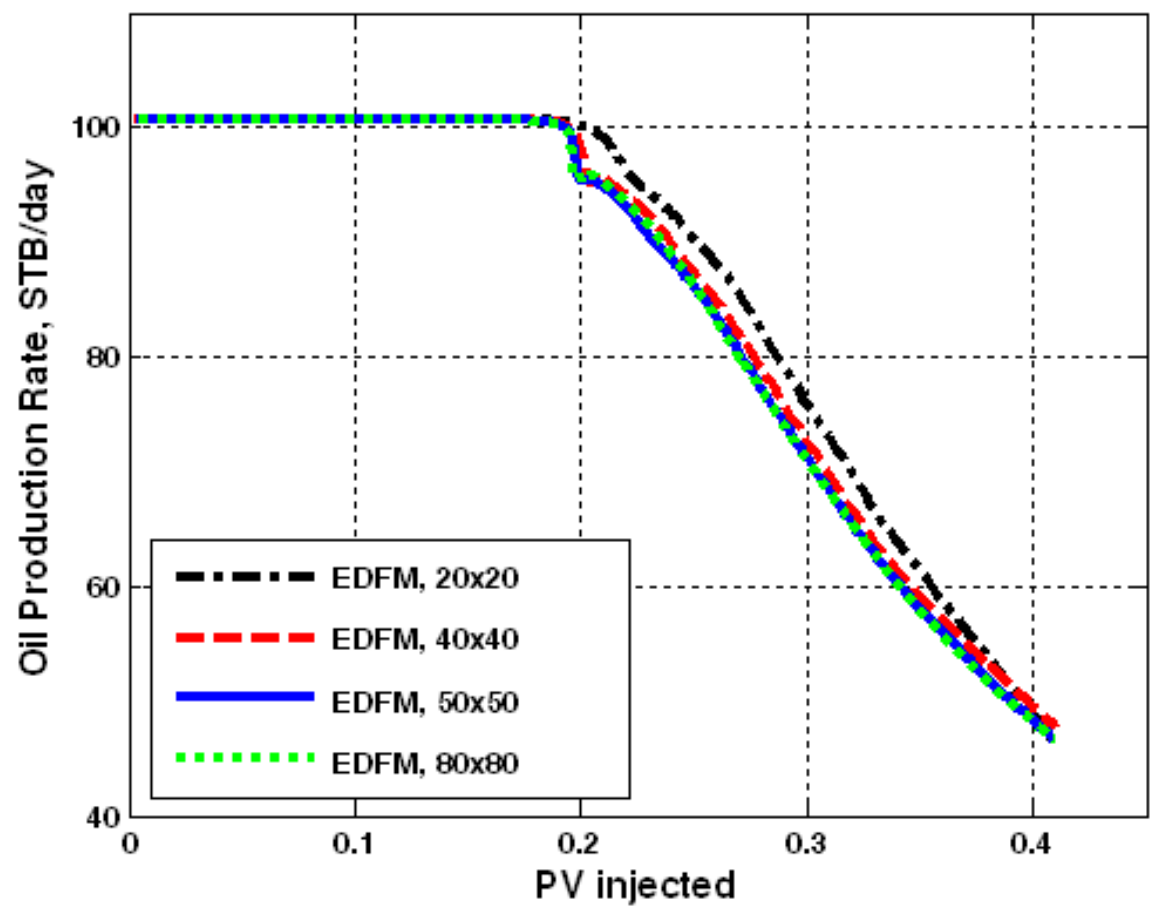

Figure 6.4: Comparison of oil production rate calculated by the EDFM approach using four grid resolutions under consideration for the model reservoir shown in Figure 6.1 in the absence of capillary pressure contrasts. 


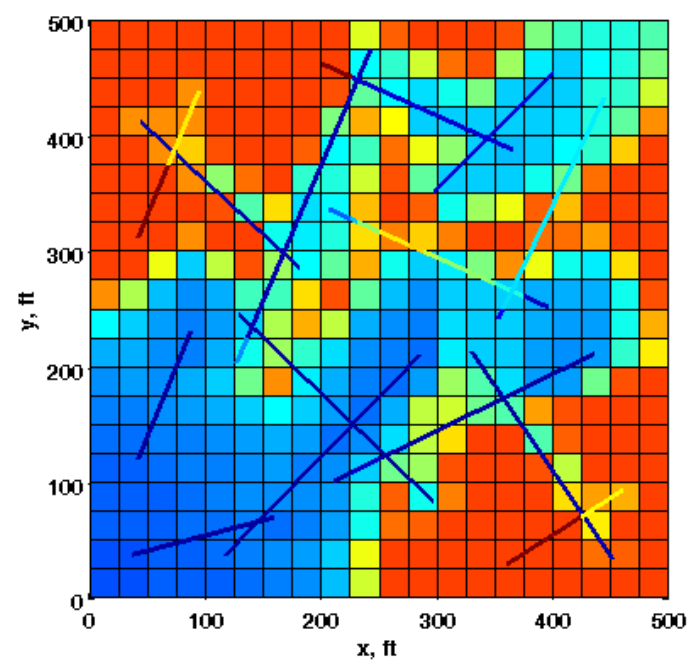

(a)

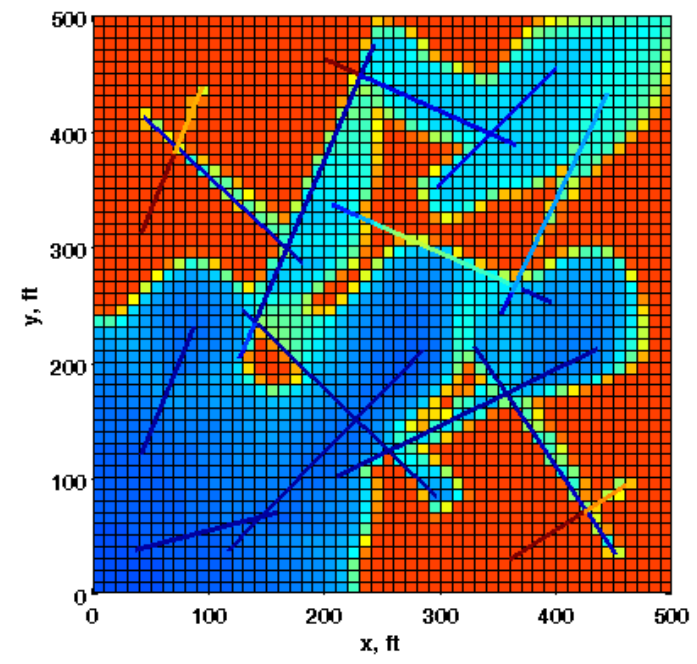

(c)

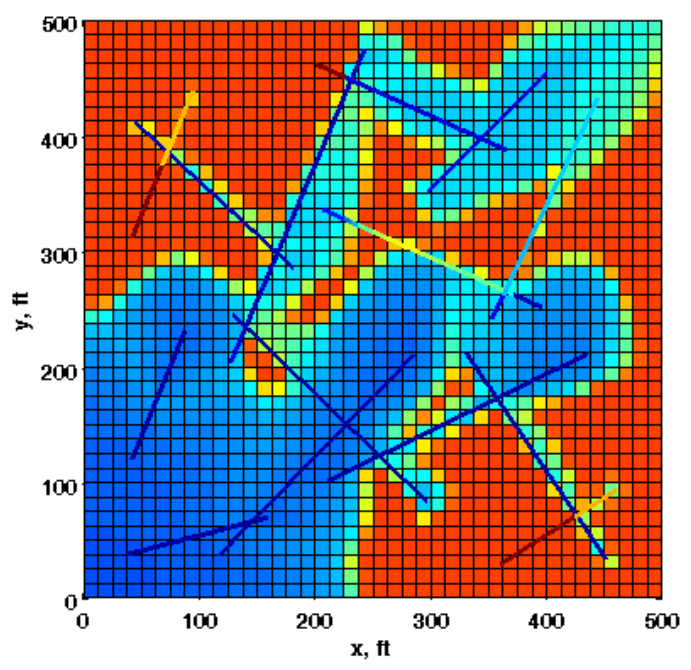

(b)

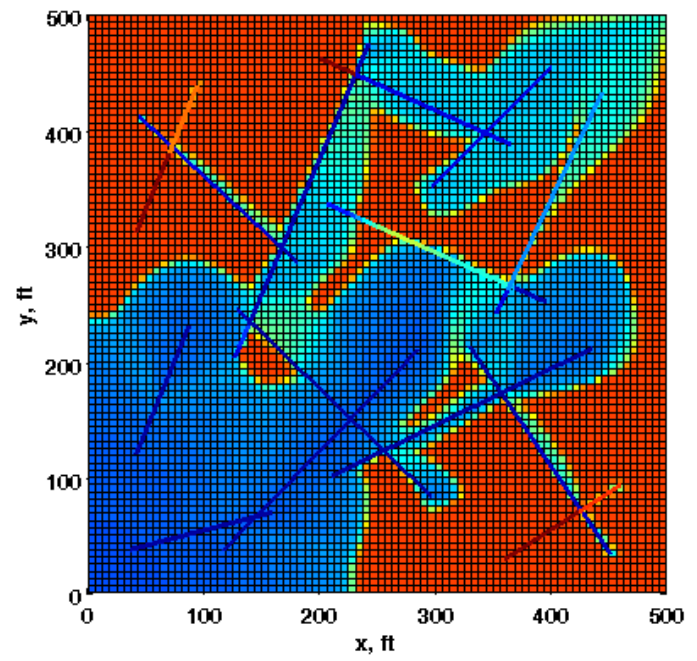

(d)

Figure 6.5: Profiles of water saturation predicted by (a) EDFM using a $20 \times 20 \times 1$ matrix grid, (b) EDFM using a 40×40×1 matrix grid, (c) EDFM using a $50 \times 50 \times 1$ matrix grid, and (d) EDFM using a $80 \times 80 \times 1$ matrix grid after 250 days of water injection (0.28 PV injected) for the model reservoir shown in Figure 6.1 in the absence of capillary pressure contrasts. 
In Section 4.3.1, we described a methodology to calculate the transmissibility between fracture control volumes and matrix gridblocks for partially-penetrating fractures. That is, when a fracture does not fully penetrate a gridblock, we first calculate the transmissibility assuming that the fracture fully penetrates the gridblock and then we correct the transmissibility for the partially-penetrating fracture. To evaluate the accuracy of this approximation, the top row in Figure 6.6 shows an enlarged region of the reservoir shown in Figure 6.1 around a partially-penetrating fracture using $40 \times 40 \times 1$ and $80 \times 80 \times 1$ matrix grids. The matrix gridblock containing the fracture segment in the $40 \times 40 \times 1$ grid is represented by four gridblocks in the $80 \times 80 \times 1$ grid, only one of which includes the fracture segment. The bottom row in Figure 6.6 shows water saturation in the mentioned gridblocks after 20 days of simulation. The water saturation in the single gridblock of the $40 \times 40 \times 1$ case is 0.337 and water saturations in the four gridblocks of the $80 \times 80 \times 1$ case are $0.579,0.284,0.281$, and 0.2 . Hence, average water saturation of the four gridblocks in the $80 \times 80 \times 1$ case is 0.336 , which is very close to the water saturation of the corresponding gridblock in the $40 \times 40 \times 1$ case. Furthermore, Figure 6.7 extends this comparison over the course of simulation, indicating that good agreement exists between water saturations calculated in both cases around the partially-penetrating fracture under consideration.

We performed an additional simulation for the model reservoir shown in Figure 6.1 to demonstrate the applicability of the EDFM approach for modeling fluid flow in heterogeneous reservoirs. Figure 6.8 presents the porosity and permeability maps assigned to a $40 \times 40 \times 1$ matrix grid, wherein porosity of matrix ranges from 0.042 to 0.158 and permeability of matrix ranges from 0.74 to 39.74 md. All other parameters are identical to those used in the previous simulations of this section. Subsequently, Figure 
6.9 shows the water saturation profiles after 150 and 250 days of water injection. The computational time for this simulation was 30.6 minutes.
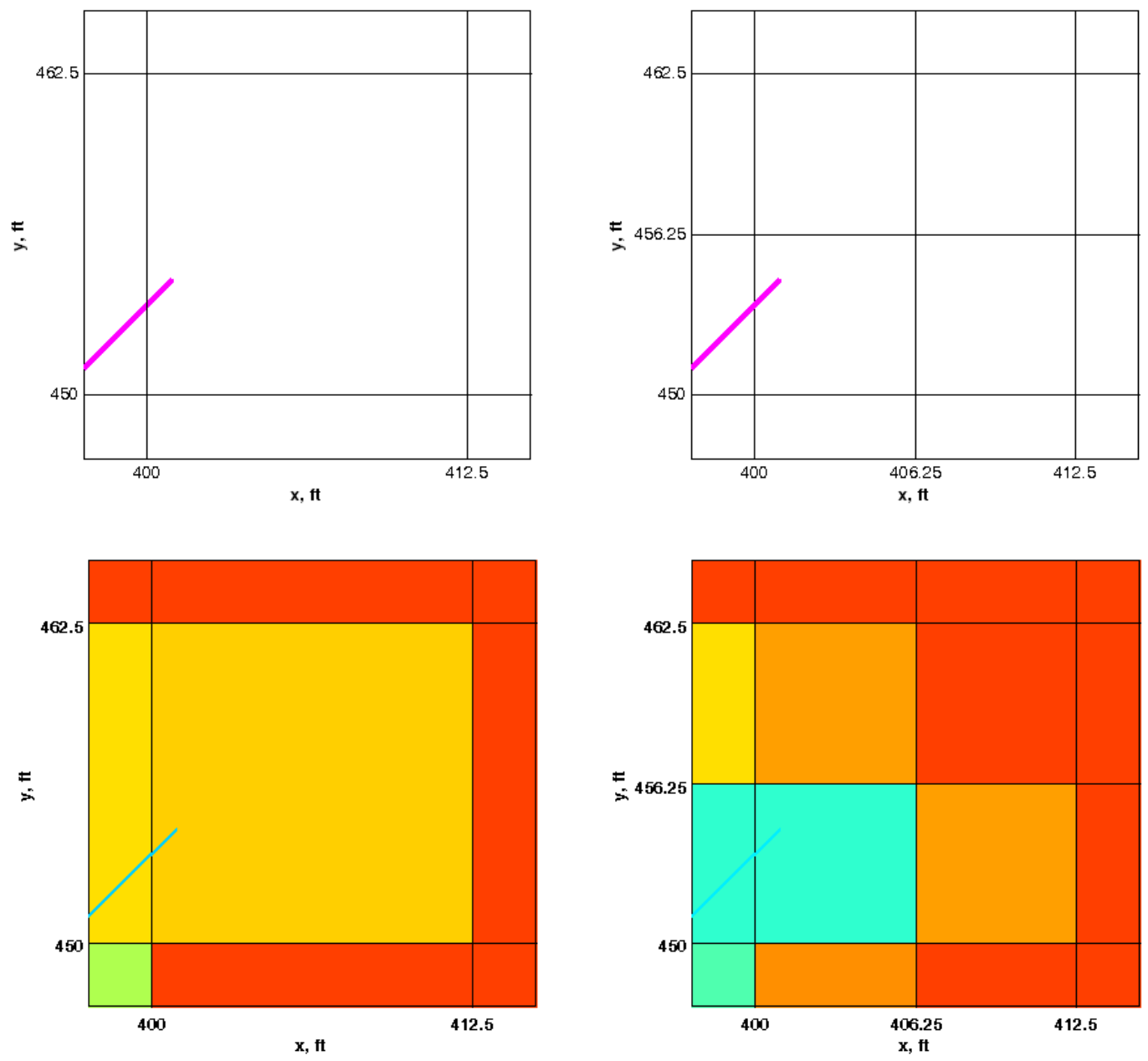

(a)

(b)

Figure 6.6: The top row presents an enlarged region of the reservoir shown in Figure 6.1 around a partially-penetrating fracture using (a) $40 \times 40 \times 1$ and (b) $80 \times 80 \times 1$ matrix grids. The bottom row shows the corresponding water saturation profiles after 20 days of water injection. 


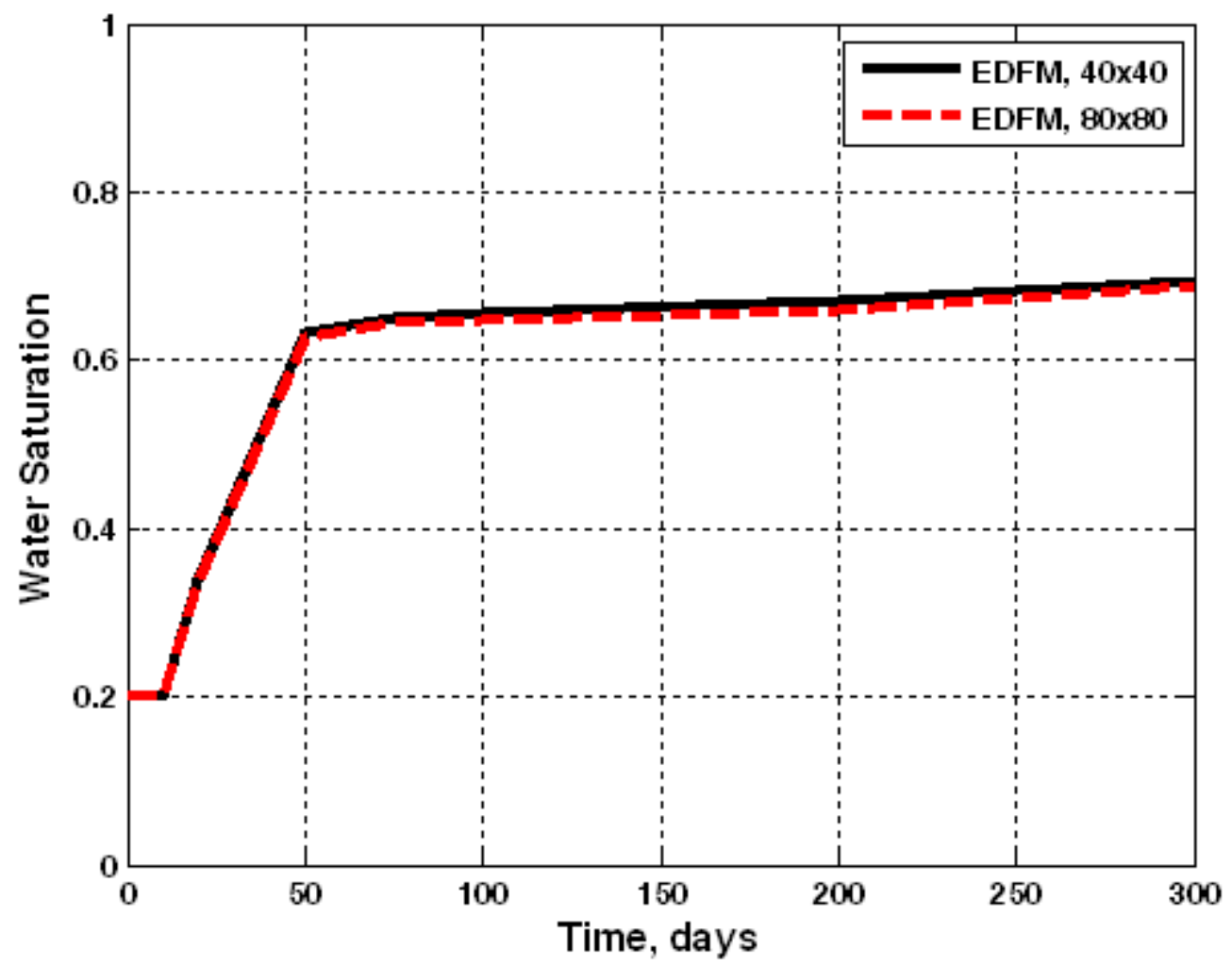

Figure 6.7: Water saturation in the gridblock shown in Figure 6.6a calculated in the $40 \times 40 \times 1$ simulation compared to the average water saturation of four gridblocks shown in Figure $6.6 \mathrm{~b}$ calculated in the $80 \times 80 \times 1$ simulation. 


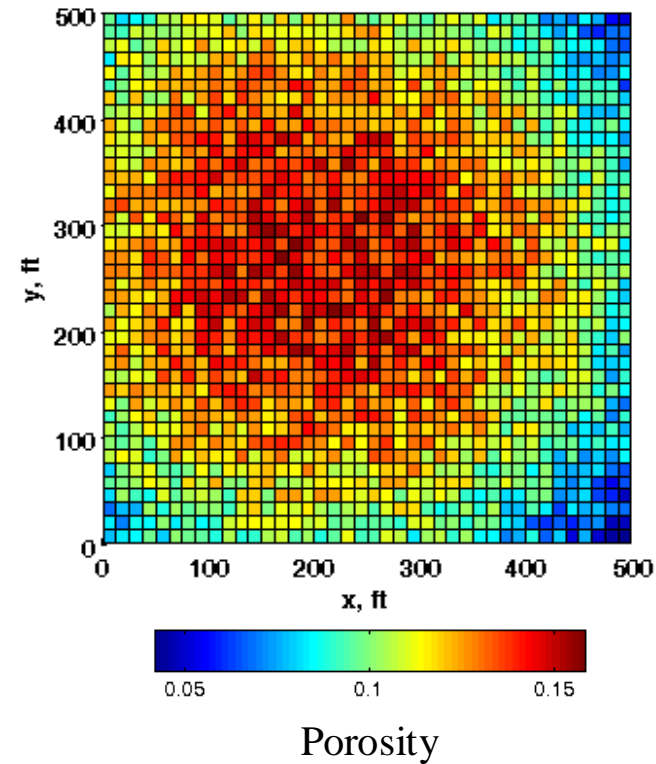

(a)

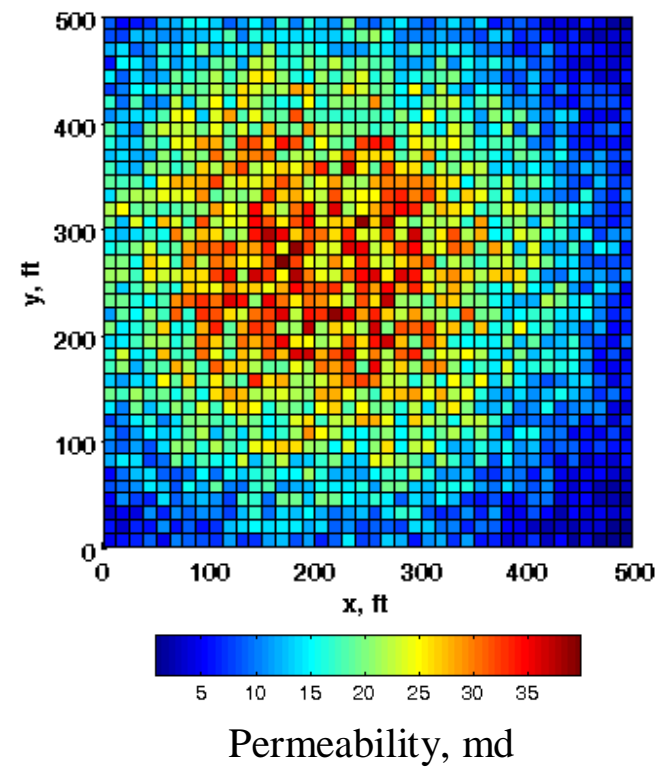

(b)

Figure 6.8: Description of (a) porosity and (b) permeability maps assigned to a $40 \times 40 \times 1$ matrix grid for the fractured reservoir shown in Figure 6.1.
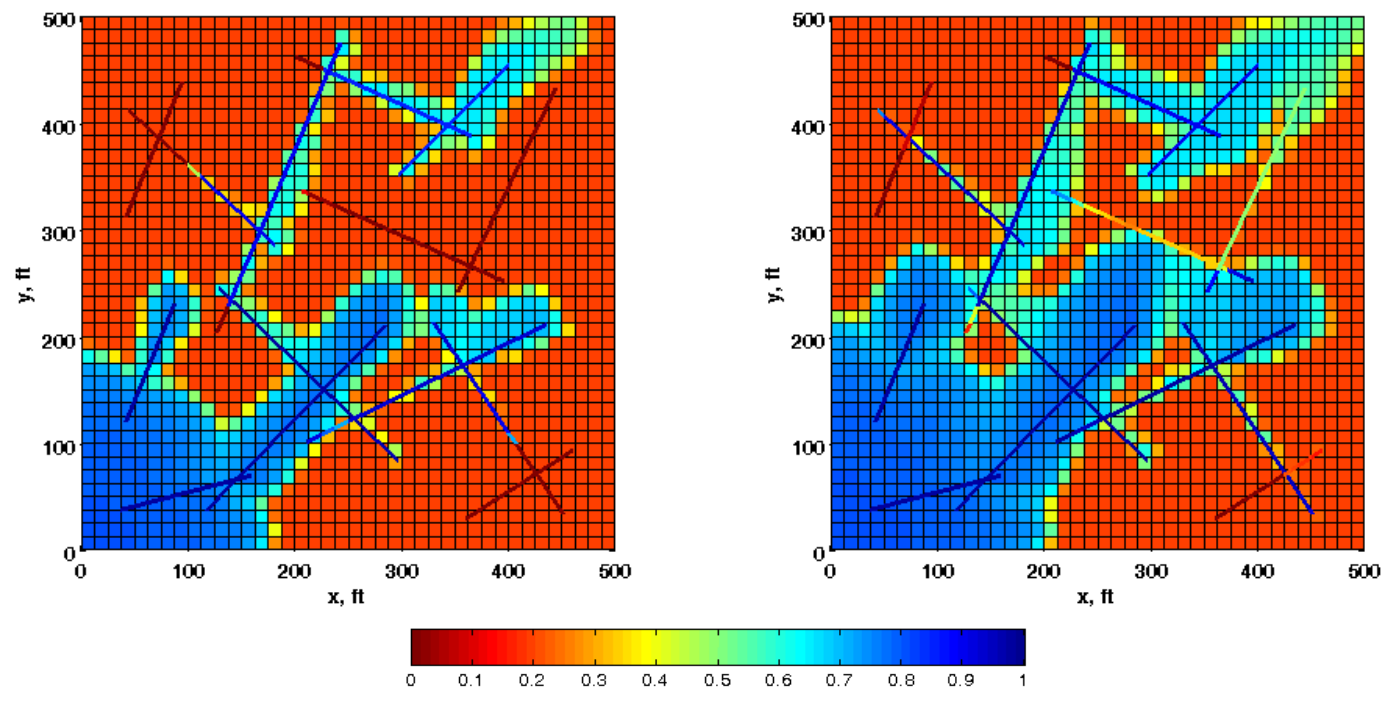

Water saturation

(a)

(b)

Figure 6.9: Water saturation profiles calculated for the heterogeneous reservoir described in Figure 6.8 at (a) 0.17 PV injected (150 days), and (b) 0.28 PV injected (250 days). 


\subsubsection{D Gas Injection (Effect of Gravity)}

Gas injection into a reservoir is examined next to demonstrate that the EDFM method can treat both compositional gas flood processes and 3D flow. Also, we investigate the effect of a fracture network on oil recovery in this example. The reservoir considered for gas injection is shown in Figure 6.10. The matrix grid is $20 \times 20 \times 2$, with cell dimensions of $25 \times 25 \times 20 \mathrm{ft}$ in the $\mathrm{x}, \mathrm{y}$, and $\mathrm{z}$ directions, respectively. The model comprises five angled macro-fractures. The dip angle of the fractures ranges from 60 to 70 degrees. An injector is located in one corner and a producer is placed in the opposite corner. Both injector and producer have vertical wellbores completed in both layers. In addition to water, six hydrocarbon components are present in this example: $\mathrm{CH}_{4}, \mathrm{C}_{3} \mathrm{H}_{8}$, $\mathrm{C}_{6} \mathrm{H}_{14}, \mathrm{C}_{10} \mathrm{H}_{22}, \mathrm{C}_{15} \mathrm{H}_{32}$, and $\mathrm{C}_{20} \mathrm{H}_{42}$, and the Peng-Robinson equation-of-state is employed for the hydrocarbon phase behavior calculations. The initial reservoir-fluid composition is $50 \% \mathrm{CH}_{4}, 3 \% \mathrm{C}_{3} \mathrm{H}_{8}, 7 \% \mathrm{C}_{6} \mathrm{H}_{14}, 20 \% \mathrm{C}_{10} \mathrm{H}_{22}, 15 \% \mathrm{C}_{15} \mathrm{H}_{32}$, and $5 \% \mathrm{C}_{20} \mathrm{H}_{42}$, while the injected-fluid composition is $98 \% \mathrm{CH}_{4}, 1 \% \mathrm{C}_{3} \mathrm{H}_{8}$, and $1 \% \mathrm{C}_{6} \mathrm{H}_{14}$. Initial reservoir pressure and the producer BHP are $2100 \mathrm{psi}$, and the injection rate is $250 \mathrm{MSCF} / \mathrm{day}$. Table 6.1 summarizes other petrophysical properties used in this example. Also, Table 6.2 describes the properties of hydrocarbon components assumed in the simulations.

Figure 6.11 shows the profiles of gas saturation after 30 and 150 days of gas injection, simulated by EDFM. The left and right panels in this figure correspond to the top and bottom computational layers, respectively. It is inferred from Figure 6.11 that significant oil is left unswept in the bottom layer, owing to gravity and presence of fractures. That is, high-permeability fractures not only expedite gas breakthrough, but also increase segregation of gas towards the top of the reservoir, leading to very low sweep efficiency in this example. 
To assess the effect of fracture network on oil recovery, we repeated the simulation in the absence of fractures. Figure 6.12 illustrates the profiles of gas saturation simulated in the absence of fractures, indicating that the bottom layer is swept by the injected gas to some extent. A remarkable reduction of oil production in the presence of fractures is evident in Figure 6.13a, where, simulated oil production rates are compared for both cases. Likewise, Figure $6.13 \mathrm{~b}$ compares the average reservoir pressure in the presence and absence of the fracture network. The computational times for the simulations in the presence and absence of fractures were 5.5 and 2.3 minutes, respectively.

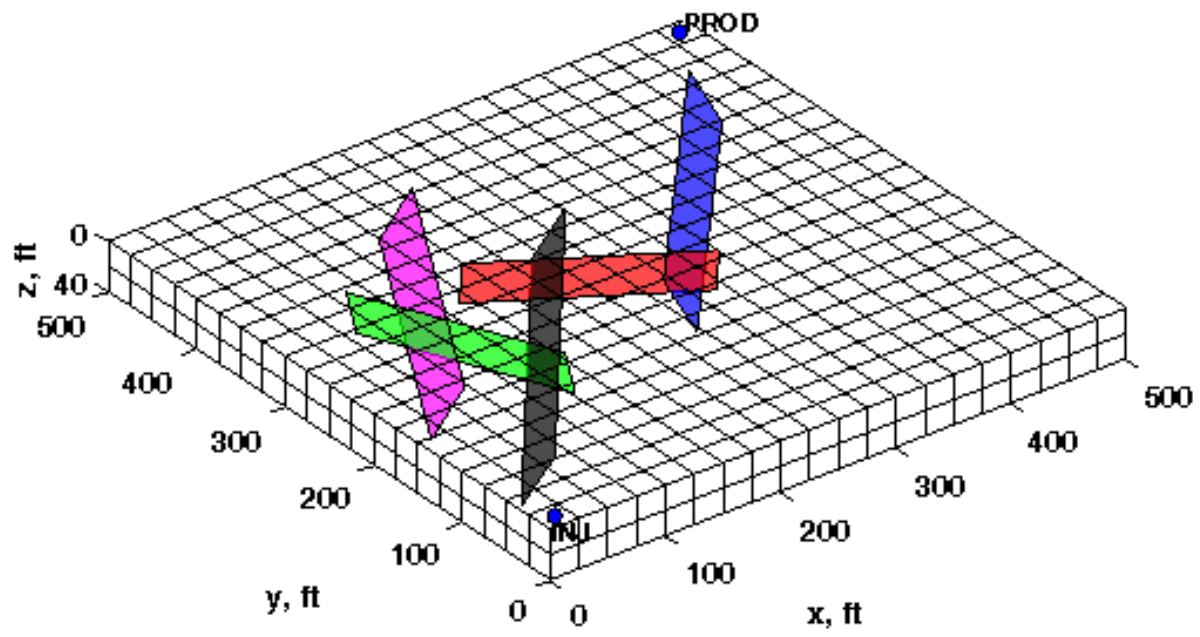

Figure 6.10: A synthetic 3D fractured reservoir $(500 \mathrm{ft} \times 500 \mathrm{ft} \times 40 \mathrm{ft})$ studied in the second example. The model reservoir comprises five inclined macrofractures. The dip angle of the fractures ranges from 60 to 70 degrees. 


\begin{tabular}{|c|c|c|c|}
\hline Variable & Value & Variable & Value \\
\hline Matrix porosity & 0.1 & Residual water saturation & 0.3 \\
\hline Matrix horizontal perm. & $20 \mathrm{md}$ & Residual oil saturation & 0.1 \\
\hline Matrix vertical perm. & $2 \mathrm{md}$ & Residual gas saturation & 0.0 \\
\hline Fracture aperture & $0.0264 \mathrm{ft}$ & Water rel. perm. endpoint & 0.4 \\
\hline Fracture horizontal perm. & $7 \times 10^{5} \mathrm{md}$ & Oil rel. perm. endpoint & 0.9 \\
\hline Fracture vertical perm. & $7 \times 10^{4} \mathrm{md}$ & Gas rel. perm. endpoint & 0.9 \\
\hline Initial water saturation & 0.17 & Water rel. perm. exponent & 3.0 \\
\hline Reservoir temperature & $160^{\circ} \mathrm{F}$ & Oil rel. perm. exponent & 2.0 \\
\hline Reservoir pressure & $2100 \mathrm{psi}$ & Gas rel. perm. exponent & 2.0 \\
\hline
\end{tabular}

Table 6.1: Petrophysical properties assumed in the simulations performed for the model reservoir shown in Figure 6.10.

\begin{tabular}{|c|c|c|c|c|c|c|}
\hline Component & $\mathbf{C H}_{\mathbf{4}}$ & $\mathbf{C}_{\mathbf{3}} \mathbf{H}_{\mathbf{8}}$ & $\mathbf{C}_{\mathbf{6}} \mathbf{H}_{\mathbf{1 4}}$ & $\mathbf{C}_{\mathbf{1 0}} \mathbf{H}_{\mathbf{2 2}}$ & $\mathbf{C}_{\mathbf{1 5}} \mathbf{H}_{\mathbf{3 2}}$ & $\mathbf{C}_{\mathbf{2 0}} \mathbf{H}_{\mathbf{4 2}}$ \\
\hline Molecular weight $(\mathrm{lb} / \mathrm{lb}-\mathrm{mol})$ & 16.0 & 44.1 & 86.2 & 142.3 & 206.0 & 282.0 \\
\hline Critical temperature $\left({ }^{\circ} \mathrm{R}\right)$ & 343.0 & 665.7 & 913.4 & 1111.8 & 1270.0 & 1380.0 \\
\hline Critical pressure (psia) & 667.8 & 616.3 & 436.9 & 304.0 & 200.0 & 162.0 \\
\hline Critical volume $\left(\mathrm{ft}^{3} / \mathrm{lb}-\mathrm{mol}\right)$ & 1.599 & 3.211 & 5.923 & 10.087 & 16.696 & 21.484 \\
\hline Acentric factor & 0.013 & 0.152 & 0.301 & 0.488 & 0.650 & 0.850 \\
\hline Parachor & 71.00 & 151.0 & 271.0 & 431.0 & 631.0 & 831.0 \\
\hline
\end{tabular}

Table 6.2: Properties of the hydrocarbon components used in the simulations performed for the model reservoir shown in Figure 6.10. 

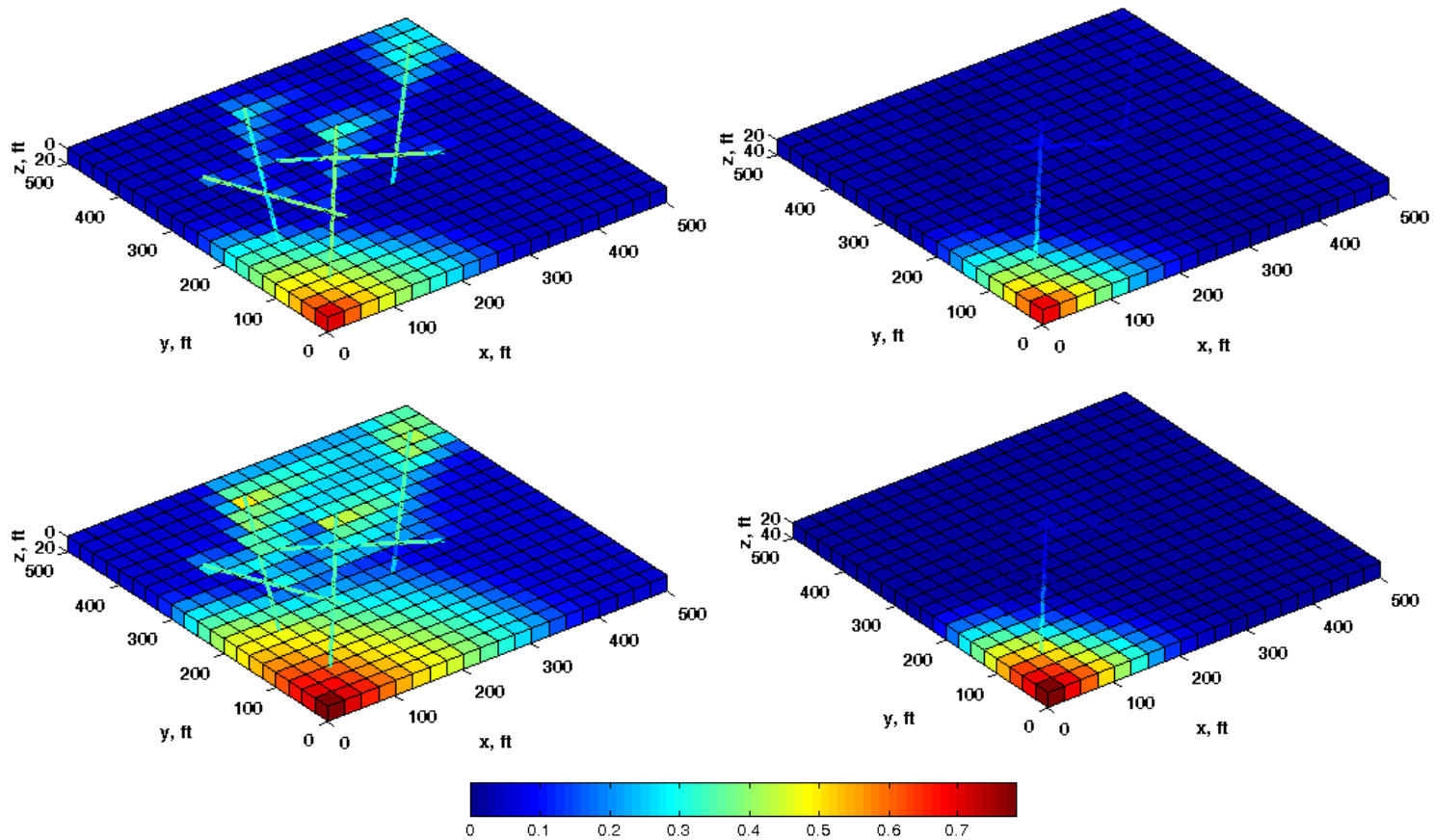

Gas saturation

(a)

(b)

Figure 6.11: Profiles of gas saturation in the (a) top layer, and (b) bottom layer of the model reservoir shown in Figure 6.10, simulated by EDFM. The first and second rows show gas saturation maps after 30 and 150 days of gas injection, respectively.
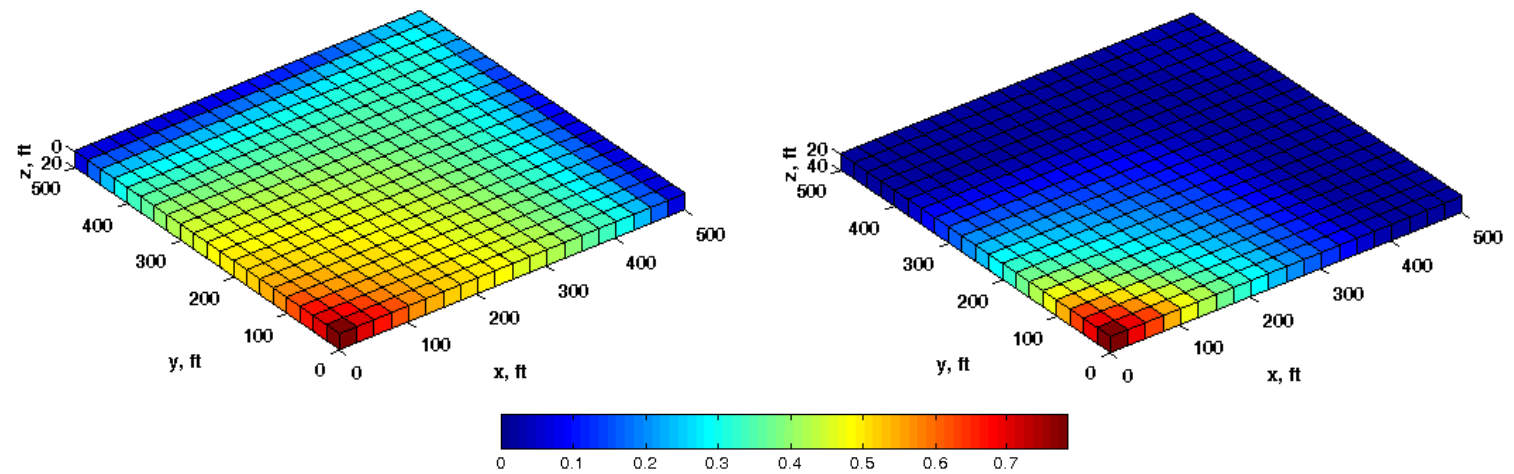

Gas saturation

(a)

(b)

Figure 6.12: Profiles of gas saturation in the (a) top layer, and (b) bottom layer of a reservoir $(500 \mathrm{ft} \times 500 \mathrm{ft} \times 40 \mathrm{ft})$ without natural fractures. The saturation maps are presented at 150 days of gas injection. 


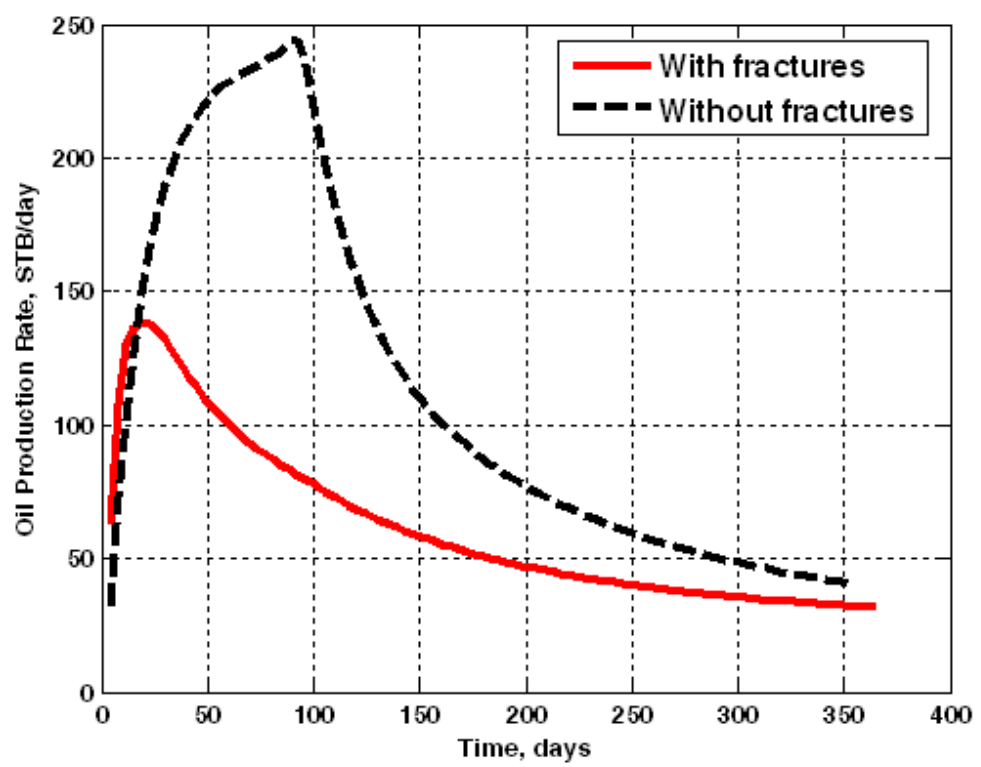

(a)

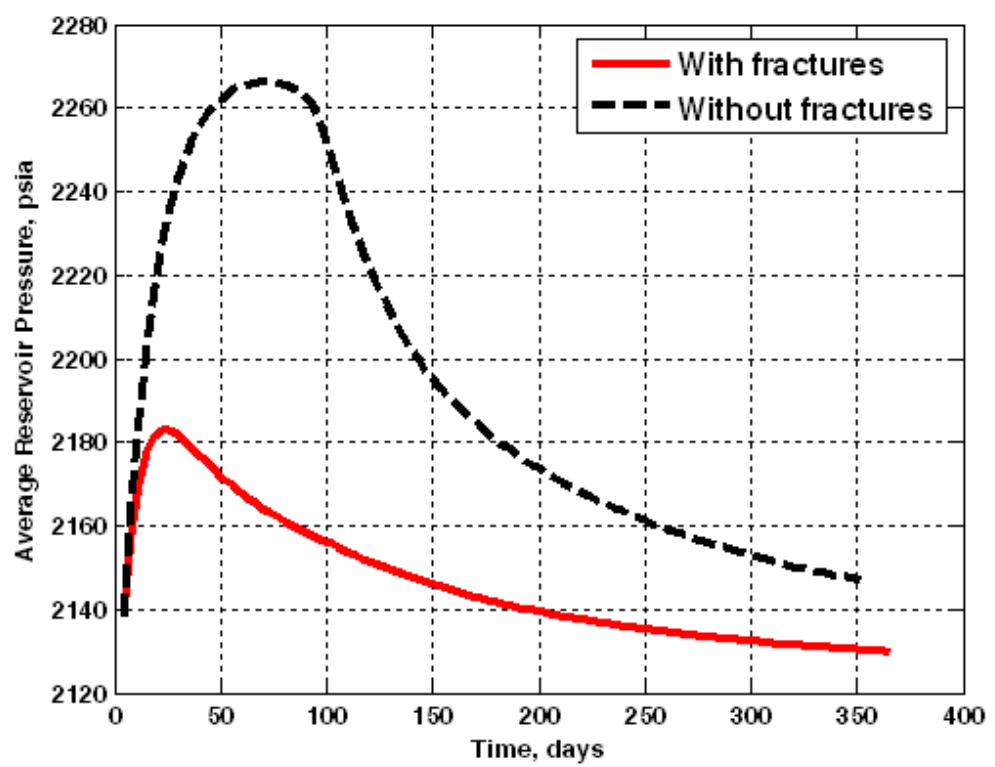

(b)

Figure 6.13: Comparison of (a) oil production rate, and (b) average reservoir pressure, over a year of gas injection into the model reservoir shown in Figure 6.10, in the presence and absence of the fracture network. 


\subsubsection{D Water Flooding}

In most simulation studies performed in the past, natural fractures were assumed to be vertical in the subsurface. However, the new implementation of the EDFM approach presented in this research provides an effective and reliable environment to model obliquely dipping fractures. Hence, the third example examines water-flooding into a 3D model reservoir shown in Figure 6.14, comprising 13 inclined fractures. The fractures are extended through different layers. We also investigate the effect of fracture inclination in this example. The dip angle of fractures ranges from 55 to 90 degrees. The matrix grid is $20 \times 20 \times 4$, with cell dimensions of $25 \times 25 \times 20 \mathrm{ft}$ in the $\mathrm{x}, \mathrm{y}$, and $\mathrm{z}$ directions, respectively. The aperture and permeability of all fractures are $0.025 \mathrm{ft}$ and $3 \times 10^{5} \mathrm{md}$, and the permeability of matrix is $15 \mathrm{md}$, and other petorphysical properties are identical to those assumed in the first example. Initial reservoir pressure and the constant producer BHP are $5000 \mathrm{psi}$, and the water injection rate is $1000 \mathrm{bbl} / \mathrm{day}$.

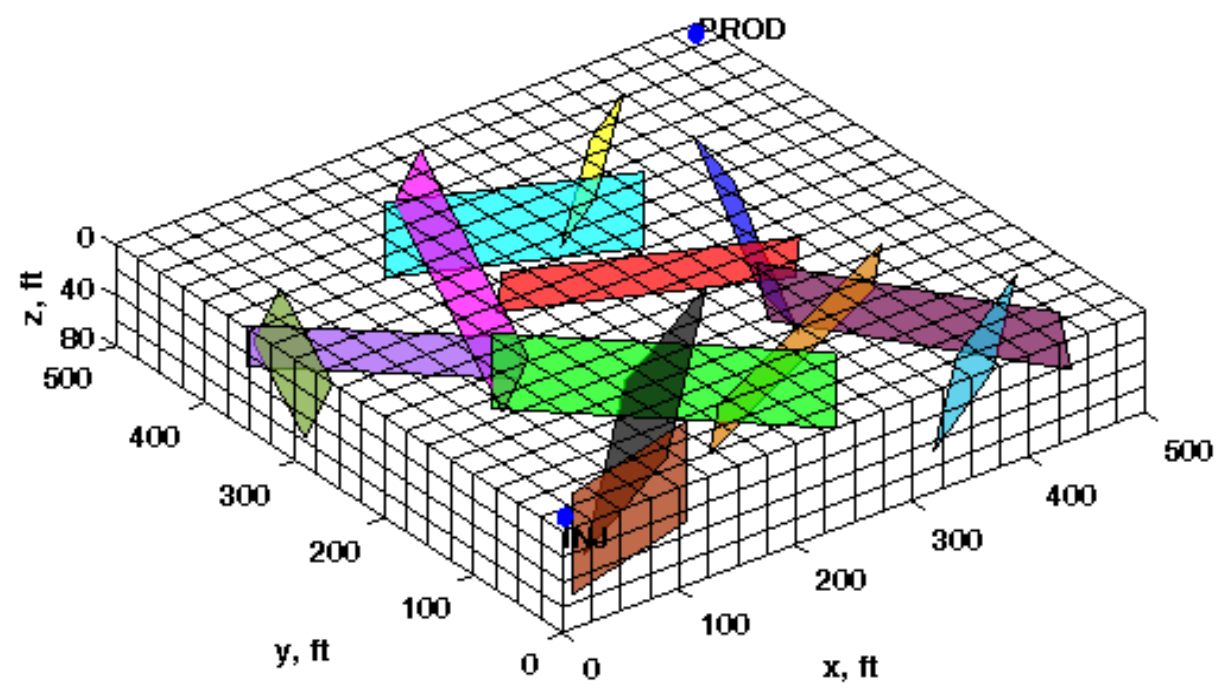

Figure 6.14: A synthetic 3D fractured reservoir $(500 \mathrm{ft} \times 500 \mathrm{ft} \times 80 \mathrm{ft})$ studied in the third example. The model reservoir comprises 13 inclined macro-fractures. The dip angle of the fractures ranges from 55 to 90 degrees. 
Figure 6.15 shows the water saturation maps in four computational layers after 80 days of water injection ( $0.225 \mathrm{PV}$ injected). It can be seen in Figure 6.15 that due to presence of inclined fractures, which are extended through different layers, the invasion patterns are different in various layers.

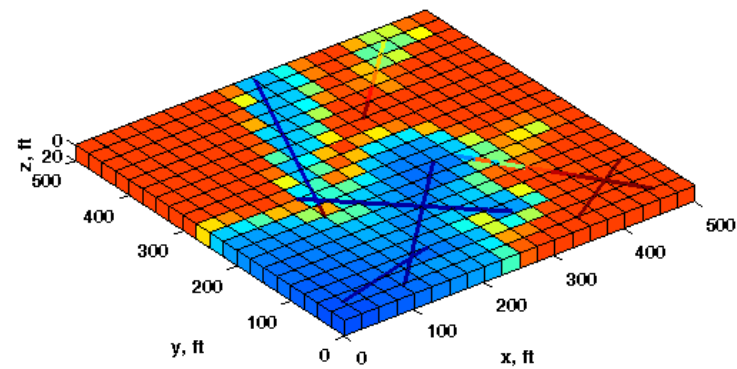

(a)

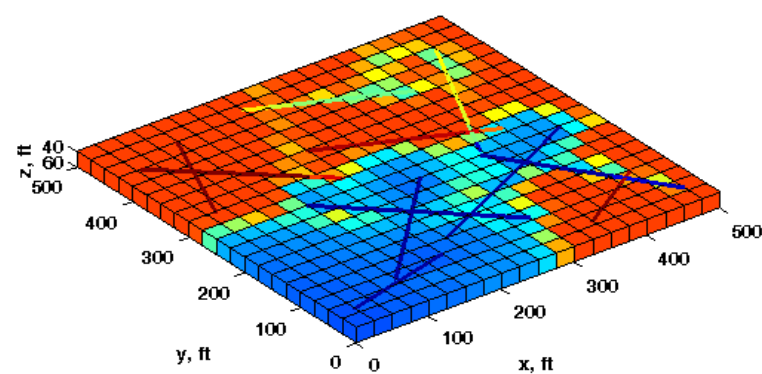

(c)

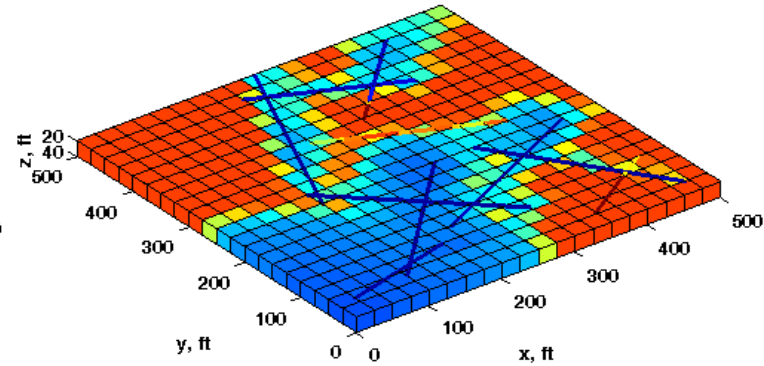

(b)

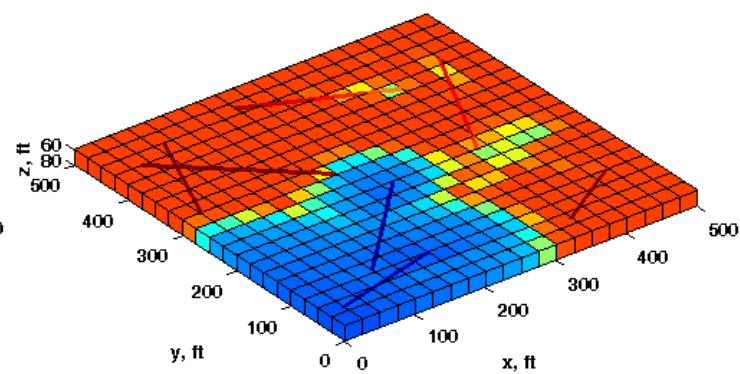

(d)

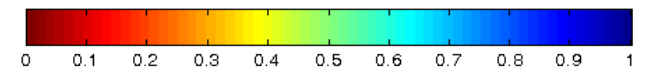

Water saturation

Figure 6.15: Profiles of water saturation in the (a) top layer, (b) second layer, (c) third layer, and (d) bottom layer of the model reservoir shown in Figure 6.14. The saturation maps are presented at 80 days of water injection. 
We also repeated the simulation assuming that fractures are all vertical. Figure 6.16 compares the oil production rate predicted by EDFM for both fracture configurations (inclined and vertical), showing different recovery curves for the scenarios under consideration. Thus, incorporating realistic fracture dip angles obtained from fracture characterization studies can be important for simulating fluid flow in naturally fractured reservoirs.

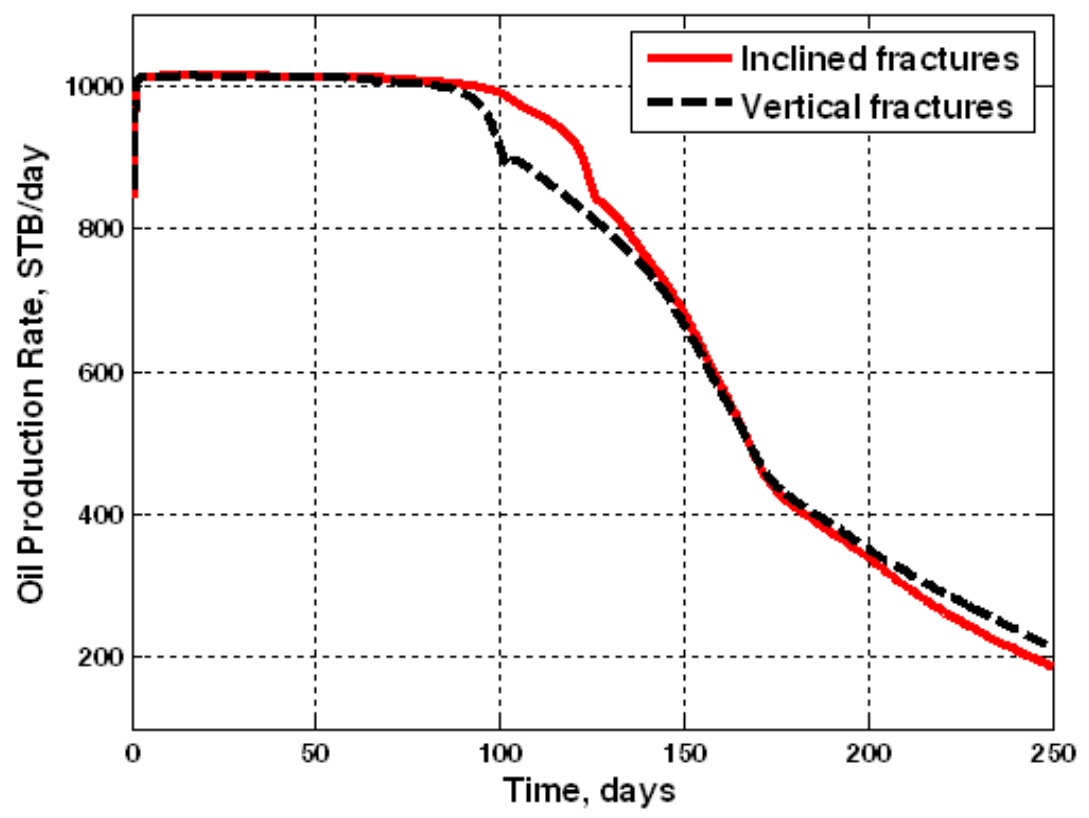

Figure 6.16: Oil production rate calculated by EDFM for the model reservoir shown in Figure 6.14, compared with the one calculated for the same reservoir wherein all fractures are assumed to be vertical. 


\subsubsection{D Primary Depletion}

The new implementation of the EDFM approach presented in this research provides an effective and reliable method to model a large number of obliquely dipping fractures. In order to show the applicability and performance of the EDFM approach for realistic simulation of fractured reservoirs, the next example examines primary depletion of a synthetic 3D model reservoir shown in Figure 6.17. The model reservoir represents a naturally fractured reservoir wherein a large number of fractures with various heights, lengths, orientations, spacings, and network connectivity are present. The reservoir dimensions are $1000 \times 1000 \times 75 \mathrm{ft}$ and a vertical well is located at the center of the reservoir and perforated along the total height of the formation. The model reservoir contains a total of 150 vertical and slanted natural fractures.

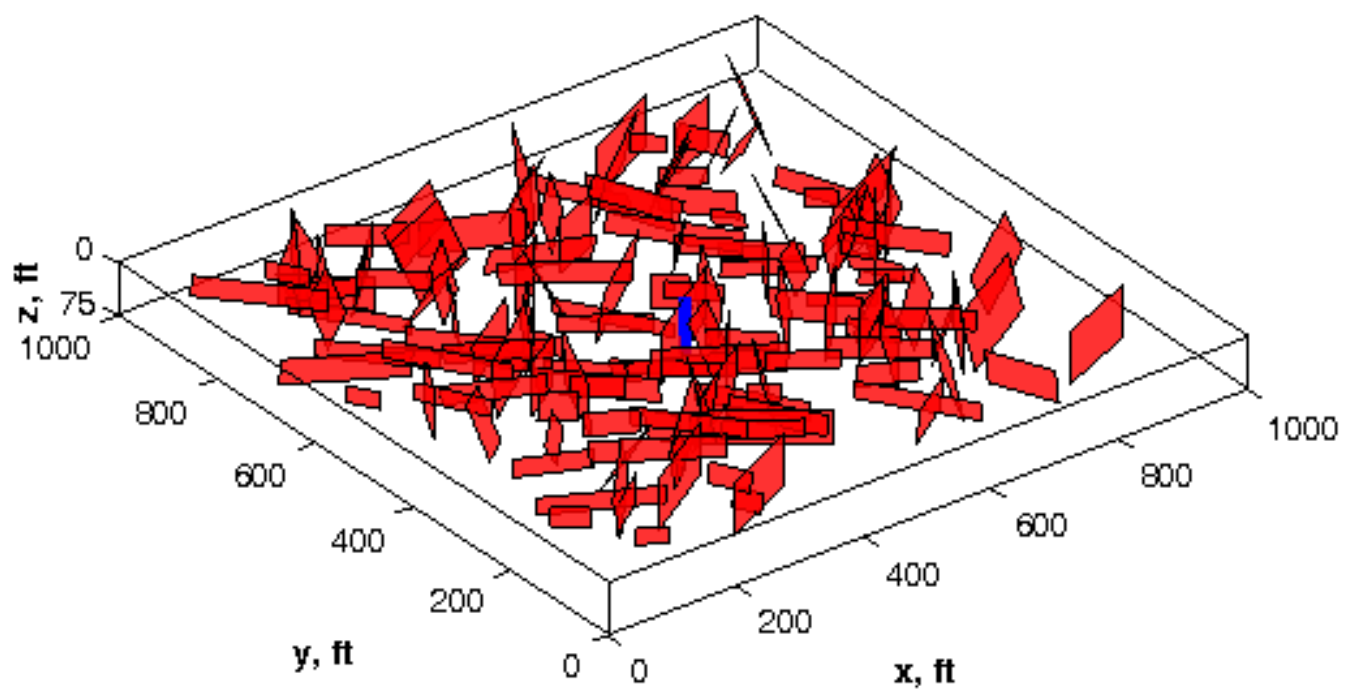

Figure 6.17: A synthetic 3D model reservoir $(1000 \times 1000 \times 75 \mathrm{ft})$ that contains a total of 150 vertical and slanted natural fractures. 
The matrix grid is $45 \times 45 \times 3$ cells in the $\mathrm{x}, \mathrm{y}$, and $\mathrm{z}$ directions, respectively. The dip angle of the fractures ranges from 60 to 90 degrees. Some fractures penetrate all three numerical layers while the rest of them penetrate only one or two layers. Moreover, fractures have different apertures, ranging from 0.52 to $1.28 \mathrm{~mm}$. The permeability of fractures, which is proportional to the square of fracture aperture, therefore ranges from 54 to 335 Darcies. The reservoir fluid is oil with specific gravity of 0.74 and viscosity of $1.5 \mathrm{cp}$, and the formation porosity and permeability are 0.26 and $0.1 \mathrm{md}$, respectively. Also, the formation temperature and the rock compressibility are $120^{\circ} \mathrm{F}$ and $5 \times 10^{-6} \mathrm{psi}^{-1}$, respectively. The water saturation in rock matrix is at the irreducible water saturation of 0.2 and the endpoint relative permeability of oil is 0.8 . The initial reservoir pressure is 4000 psi and the vertical well produces at a constant bottomhole pressure of 2000 psi, which is above the bubble point pressure.

Figure 6.18 shows the pressure profiles after 150 days of production, computed by the EDFM approach. Pressure profiles in all three numerical layers are presented in Figure 6.18, depicting different pressure depletion patterns in various layers because of the presence of randomly-generated fractures. Also, Figure 6.19 shows the oil production rate and average reservoir pressure over two years of production. The computational time of the simulation was 3.31 hours. 


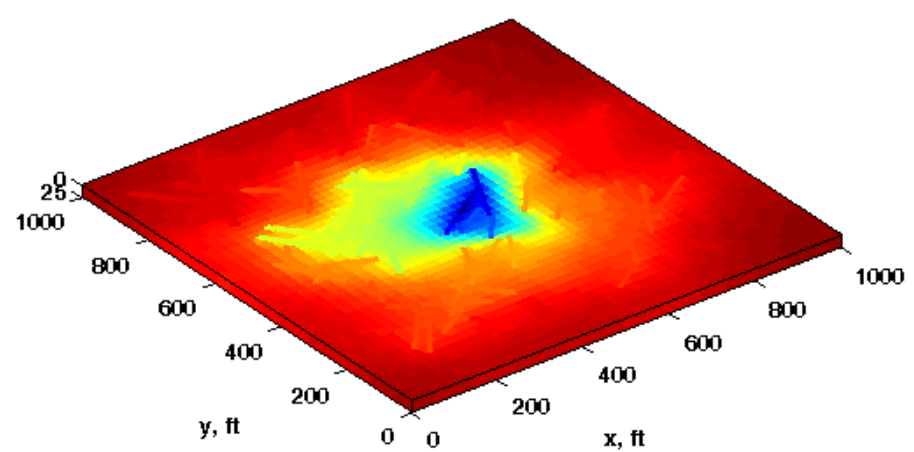

(a)

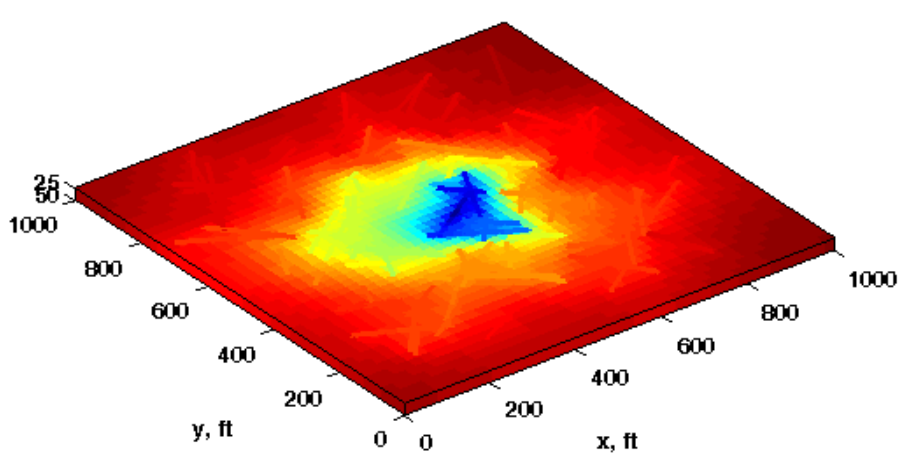

(b)

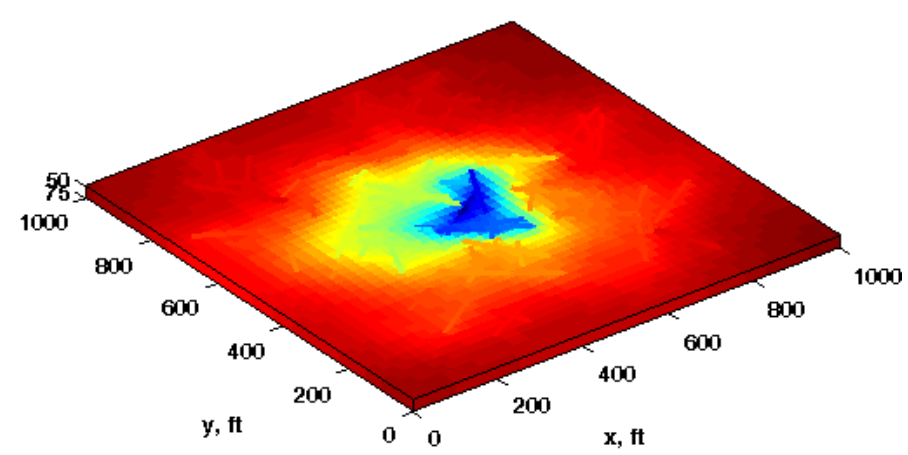

(c)

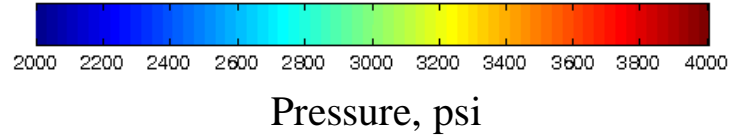

Figure 6.18: Profiles of pressure after 150 days of production in the (a) top, (b) middle, and (c) bottom numerical layers of the model reservoir shown in Figure 6.17 . 


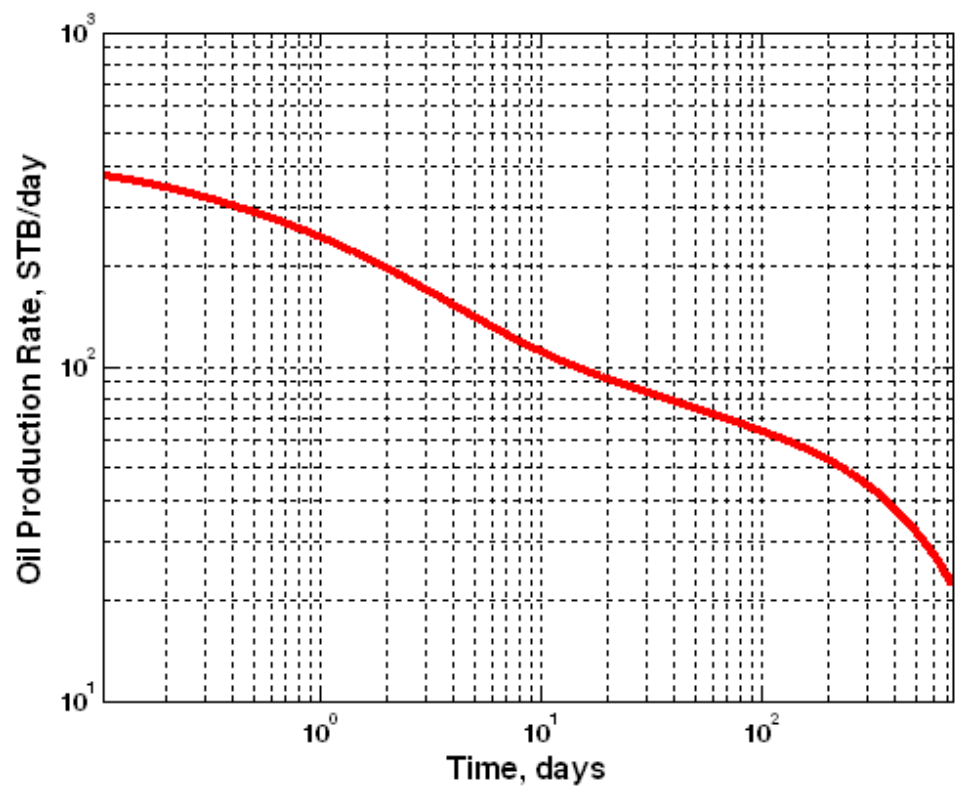

(a)

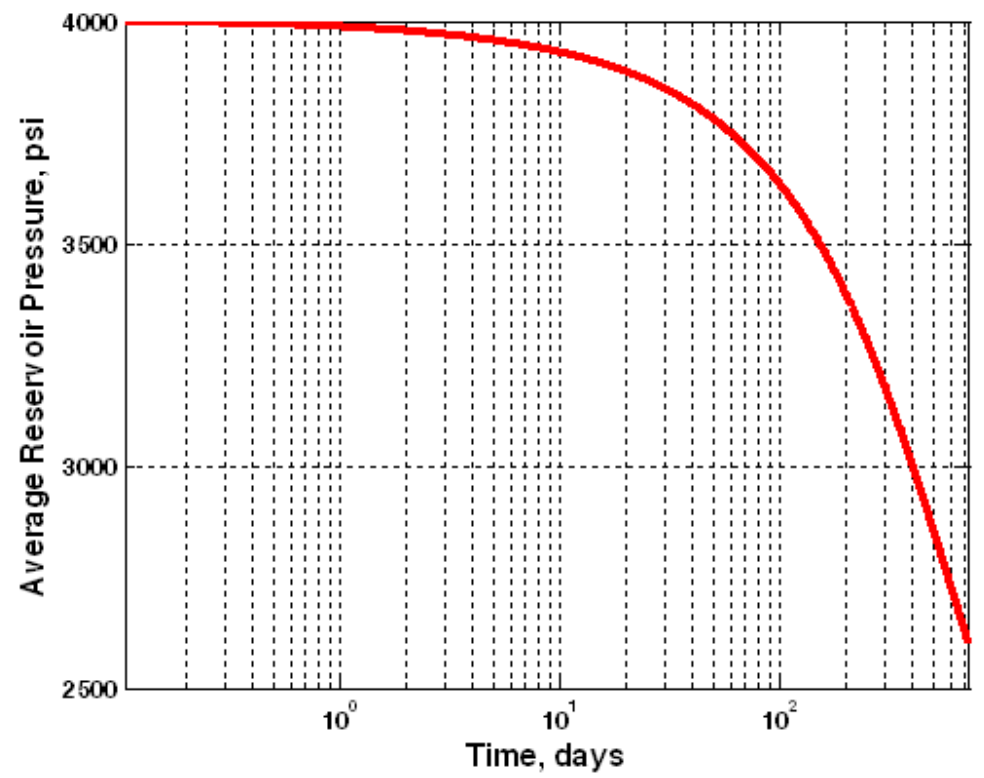

(b)

Figure 6.19: History of (a) oil production rate, and (b) average reservoir pressure over two years of production for the model reservoir shown in Figure 6.17. 


\subsection{Comparison of EDFM to An UnSTRUCTURED DFM AND A DUAL PERMEABILITy MODEL}

This section presents a study performed during a summer internship with Chevron Energy Technology Company (Chevron ETC). The financial support for this work and the permission for its publication are gratefully appreciated.

As previously described, natural fracture systems commonly show an asymmetrical distribution of fracture sizes, with numerous small fractures and fewer large fractures (Odling et al., 1999). It is not practical to treat small fractures explicitly in discrete fracture simulations. Hence, Lee et al. (2001) presented a hierarchical modeling approach for addressing flow in NFRs. In this approach, small fractures are represented by their aggregate effective properties and the large-scale fractures are modeled explicitly. Small fractures are less than the longest dimension of a grid cell in length while the large, explicit fractures are those longer than grid cells. Two discrete fracture modeling methodologies, developed at Chevron ETC, are designed to model the largest fluid conductive fractures in the system.

\subsubsection{Chevron Discrete Fracture Models}

Two discrete fracture models, based on different, independent techniques, have been developed for simulating the flow behavior of naturally fractured reservoirs. The unstructured discrete fracture model (USDFM) is based on unstructured gridding with local refinement near fractures (Hui and Mallison, 2009). Alternatively, the embedded discrete fracture model (EDFM) directly incorporates fractures in a conventional, structured grid (Li and Lee, 2008). Both modeling methods allow large-scale, beddingnormal fractures to be included into sector and full-field models. In both techniques, fractures are approximated by planar rectangles that are orthogonal to bedding, but have 
arbitrary orientation in the horizontal plane. For simplicity of geometric design, both models are only implemented for vertical fractures.

The USDFM uses the discretization approach proposed by Karimi-Fard et al. (2004). The method is based on unstructured gridding and employs the so-called lower dimensional approach to DFM gridding where the rock matrix is modeled by 3D polyhedral cells and the fracture network is represented by a subset of the $2 \mathrm{D}$ interfaces separating grid cells. The material balance for each control volume requires the knowledge of neighboring control volumes (a connectivity list) and the transmissibility associated with each connection in order to compute fluid exchange between neighboring control volumes. A two-point flux approximation is applied in the transmissibility calculations.

Grid generation is a critical step for all DFMs that rely on unstructured gridding. Available general-purpose grid-generation tools are not well suited for gridding fracture networks. Addressing this limitation, Mallison et al. (2010) presented new gridgeneration algorithms for simulating fluid flow in NFRs. Moreover, Mallison et al. (2010) argue that there is inherent uncertainty in the precise position and geometry of fractures in an NFR and hence chose not to impose an exact agreement of fracture geometry between the geologic and flow-simulation models. Instead, their gridgeneration algorithms are designed to capture only geometric features that are larger than the specified grid resolution. That is, compromises are made between maintaining good cell quality and honoring fracture geometry. These gridding tools make it possible to construct multiple models during the course of a single simulation study. 


\subsubsection{Grid Sensitivity of DFMs}

As grid orientation can affect the accuracy of modeling, we investigated the sensitivity of the DFMs to grid design. Figure 6.20a shows a synthetic 2D reservoir containing 12 discrete fractures. Figures $6.20 \mathrm{~b}$ and $6.20 \mathrm{c}$ show the same fracture configuration relative to a coordinate axes rotated $22.5^{\circ}$ and $45^{\circ}$ counter-clockwise, respectively, about the $\mathrm{z}$ axis. Grid cells outside of the outer circle in each model are inactive. Petrophysical parameters and fluid properties are summarized in Table 6.3. Initial conditions and wellbore operating constraints for this test are also described in Table 6.3. We simulate water injection for each grid orientation using both EDFM and USDFM.

EDFM utilizes a fixed Cartesian grid. Therefore, the only relative differences between model realizations are the location of wells and fractures. In contrast, the simulation grid for the USDFM approach is constructed on a coarse background structured grid that is refined locally near the fractures and adjusted to approximate the fracture geometry. These local changes vary depending on the location and orientation of fractures within the background Cartesian grid. Hence, the three choices for the coordinate axes lead to three distinct grids. We used the same resolution for the EDFM Cartesian grid and the USDFM background grid.

Figure 6.21 shows the water saturation maps predicted by EDFM approach for three grid orientations. We can see that the saturation maps are in good agreement with each other. That is, there is no appreciable effect of grid orientation on EDFM performance. This observation helps confirm that the model parameters of EDFM are accurate in cases where fractures are not aligned with the grid. Likewise, Figure 6.22 presents the water saturation maps calculated by USDFM for the three cases. Water saturation maps in Figures $6.22 \mathrm{~b}$ and $6.22 \mathrm{c}$ corresponded to the rotation of coordinate 
axes by $22.5^{\circ}$ and $45^{\circ}$, respectively, are very similar to those simulated by the EDFM approach. However, a difference is observed in the water saturation map in Figure 6.22a. Due to approximations made during grid generation, two fractures in the $0^{\circ}$ USDFM model have established a direct connection that does not exist in the other models. This "snapping" together of fractures can occur when they are offset by distances less than the grid resolution. This issue can be resolved through refinement of the grid.

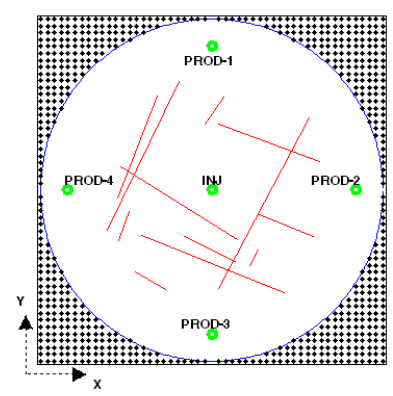

(a)

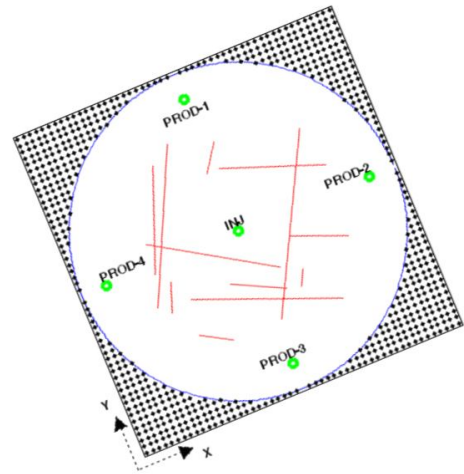

(b)

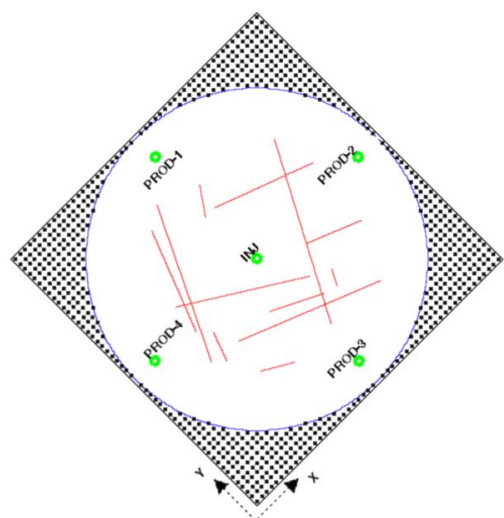

(c)

Figure 6.20: (a) A synthetic 2D $(5100 \mathrm{ft} \times 5100 \mathrm{ft})$ reservoir used to evaluate the sensitivity of DFMs to grid orientation. Panels (b) and (c) show the same fractured realization overlain on rotated coordinate axes. The coordinate $\mathrm{x}$ and y axes in Panels (b) and (c) are rotated $22.5^{\circ}$ and $45^{\circ}$ counter-clockwise, respectively, with regard to the coordinate axes in Panel (a). The reservoir model is cylindrical with the thickness of $20 \mathrm{ft}$. 


\begin{tabular}{|c|c|c|c|}
\hline Variable & Value & Variable & Value \\
\hline Matrix porosity & 0.03 & Injector BHP & $14000 \mathrm{psi}$ \\
\hline Matrix permeability & $1 \mathrm{md}$ & Producer BHP & $10000 \mathrm{psi}$ \\
\hline Fracture aperture & $0.5 \mathrm{~mm}$ & Reservoir pressure & $10000 \mathrm{psi}$ \\
\hline Fracture permeability & $2.11 \times 10^{7} \mathrm{md}$ & Reservoir temperature & $228^{\circ} \mathrm{F}$ \\
\hline Depth & $14765 \mathrm{ft}$ & Initial water saturation & 0.15 \\
\hline
\end{tabular}

Water-oil relative permeability and capillary pressure data for the matrix

\begin{tabular}{|c|c|c|c|}
\hline $\boldsymbol{S}_{\boldsymbol{w}}$ & $\boldsymbol{k}_{\boldsymbol{r w}}$ & $\boldsymbol{k}_{\boldsymbol{r o}}$ & $\boldsymbol{P}_{\text {cow }}, \mathrm{psi}$ \\
\hline 0.15 & 0 & 1 & 120 \\
\hline 0.20 & 0.013 & 0.828 & 50 \\
\hline 0.25 & 0.025 & 0.658 & 19.230 \\
\hline 0.30 & 0.035 & 0.520 & 17.307 \\
\hline 0.35 & 0.045 & 0.394 & 15.384 \\
\hline 0.40 & 0.055 & 0.240 & 13.461 \\
\hline 0.45 & 0.069 & 0.137 & 11.538 \\
\hline 0.50 & 0.089 & 0.090 & 9.615 \\
\hline 0.55 & 0.112 & 0.061 & 7.692 \\
\hline 0.60 & 0.135 & 0.037 & 5.769 \\
\hline 0.65 & 0.162 & 0.017 & 3.846 \\
\hline 0.70 & 0.200 & 0.001 & 1.923 \\
\hline 0.75 & 0.250 & 0 & 0 \\
\hline 0.80 & 0.315 & 0 & 0 \\
\hline 0.85 & 0.402 & 0 & 0 \\
\hline 0.90 & 0.523 & 0 & 0 \\
\hline 0.95 & 0.704 & 0 & 0 \\
\hline 1.00 & 1 & 0 & 0 \\
\hline
\end{tabular}

Table 6.3: Petrophysical properties and wellbore operating constraints assumed in the simulations performed for the model reservoirs shown in Figure 6.20. 


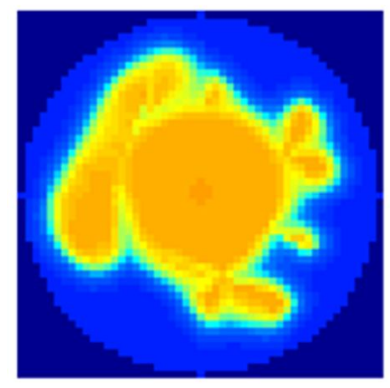

(a)

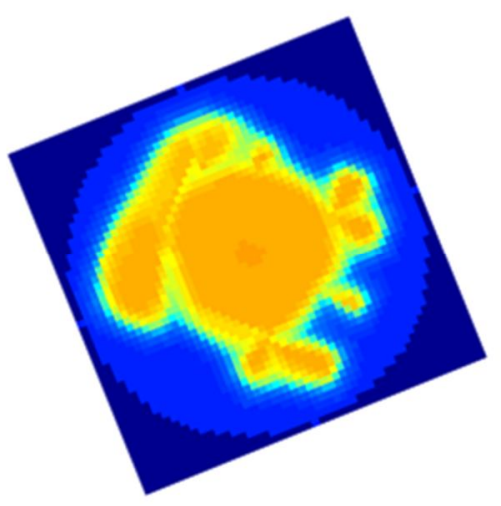

(b)

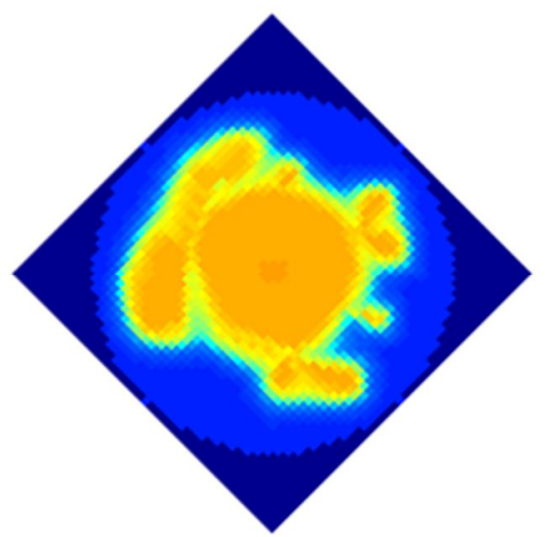

(c)

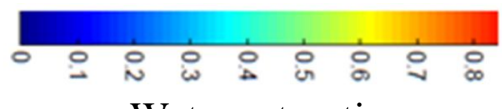

Water saturation

Figure 6.21: Water saturation maps simulated by EDFM for three grid orientations shown in Figure 6.20. Panels (a), (b), and (c) correspond to similar panels in Figure 6.20.

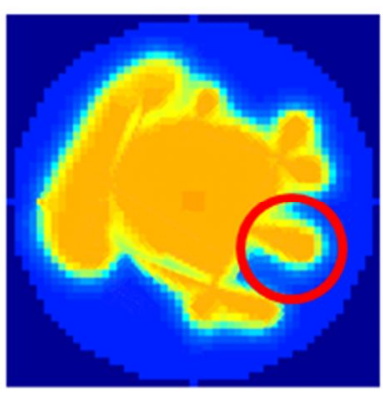

(a)

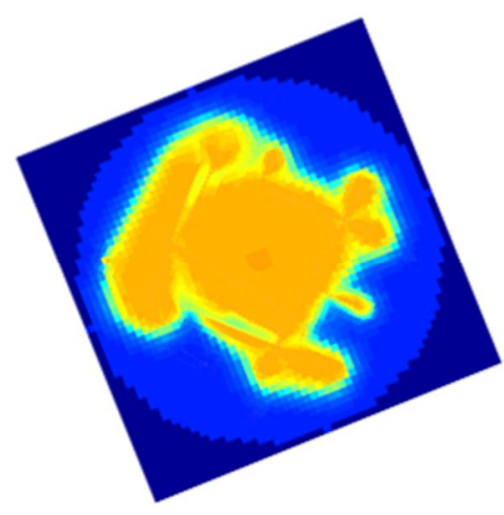

(b)

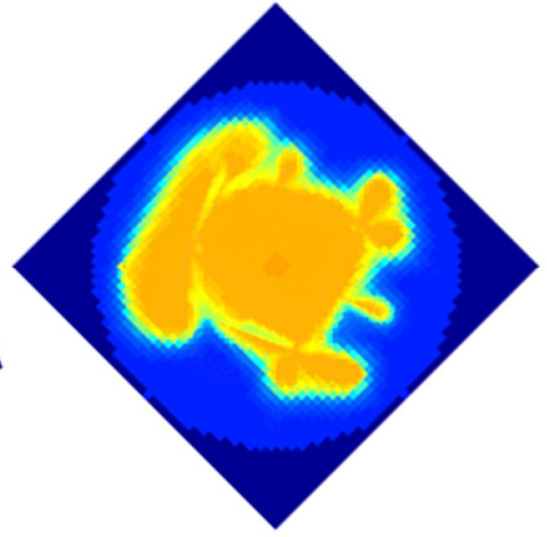

(c)

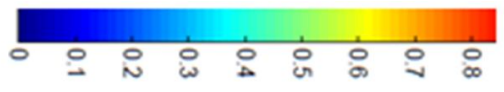

Water saturation

Figure 6.22: Water saturation maps simulated by USDFM for three grid orientations shown in Figure 6.20. Panels (a), (b), and (c) correspond to similar panels in Figure 6.20. The red circle in Panel (a) indicates the region where water saturation map differs slightly from those shown in Panels (b) and (c). 


\subsubsection{Dual Permeability Modeling}

Dershowitz et al. (2000) proposed a technique to integrate discrete fracture models with dual porosity models. Parameters used for dual porosity simulations are derived through more geologically realistic discrete fracture networks in order to reflect the connectivity, heterogeneity, and anisotropy of fractured reservoirs more accurately. They derived directional fracture system permeability from stochastically generated fracture models, and the calculated flow parameters were then used as a basis for dual porosity simulation. This approach provides an opportunity to improve dual porosity simulations. In the subsequent sections, we compare the discrete fracture models with classical dual continuum models for solving various fluid-flow problems in complex fractured reservoirs. We focus on dual permeability modeling rather than dual porosity because allowing flow to occur between matrix gridblocks can provide a more accurate simulation of NFRs than dual porosity. Moreover, to improve dual continuum simulations, we extend the approach proposed by Dershowitz et al. (2000) to dual permeability modeling as described below.

Our dual permeability simulation entails the calculation of effective fracture porosity and effective directional permeability tensor for each cell containing fractures. This improves modeling of connectivity and heterogeneity in fractured porous media. Fracture porosity is the volume of fractures embedded in a cell divided by its bulk volume. Furthermore, a single-phase upscaling tool is used to compute fracture system directional (tensor) permeability from fracture and matrix permeabilities and their interaction. The details of calculation of effective directional permeability are presented in Lee et al. (2000). In order to simulate large (multi-cell) discrete fractures, active dual permeability cells are arranged stepwise to form connected linear pathways. 


\subsubsection{Comparison of DFMs and Dual Permeability}

In this section, we compare the discrete fracture models (USDFM and EDFM) and the proposed dual permeability approach for modeling fluid flow in complex fractured reservoirs. We consider two important NFR recovery mechanisms in our tests: capillary imbibition during water-flooding and gravity drainage in oil-gas systems. We evaluate two different reservoir models for the comparison: a sparsely fractured reservoir and an irregular, anisotropic fractured reservoir.

\subsubsection{2D Sparsely Fractured Reservoir, Water Injection}

The first reservoir model evaluated in this section is the synthetic $2 \mathrm{D}$ reservoir previously considered for the grid-orientation sensitivity test (Figure 6.20a). This model represents a sparsely fractured reservoir containing only 12 discrete fractures. Figures 6.21a and 6.22a show water saturation maps computed by two DFMs. Figure 6.23 shows a water saturation map predicted by the dual permeability model using the same Cartesian grid as that of the EDFM. Differences between water saturation maps are relatively small. Likewise, Figure 6.24 compares the field water-cut with respect to time calculated by the three models, exhibiting a good agreement among the three models. Consequently, the dual permeability technique successfully reproduced the results of the DFMs. 


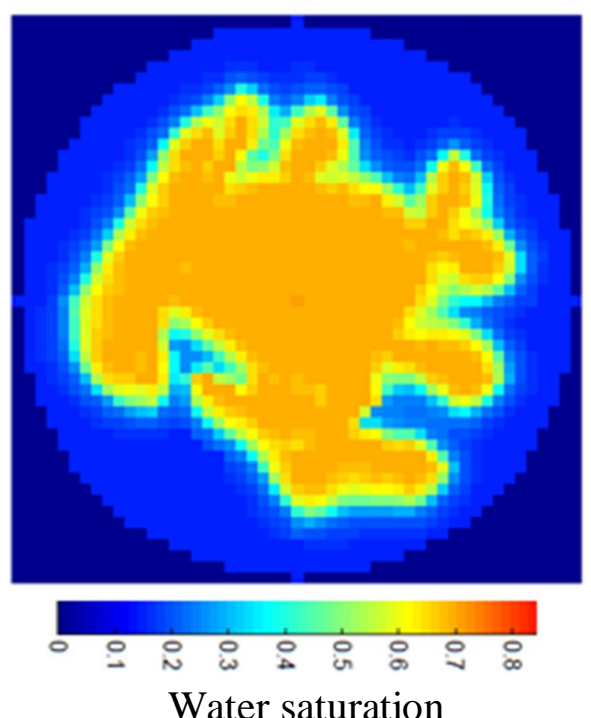

Figure 6.23: Water saturation map simulated by the systematic dual permeability model for the sparsely fractured reservoir of Figure 6.20a.

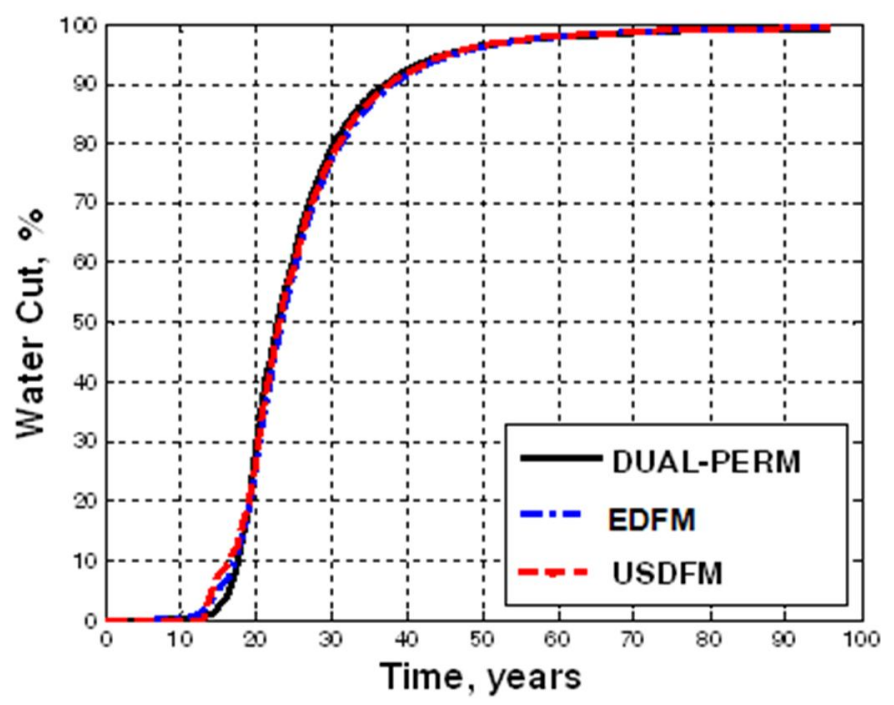

Figure 6.24: Comparison of field water-cut calculated by dual permeability, EDFM, and USDFM models for the sparsely fractured reservoir of Figure 6.20a. 


\subsubsection{3D Sparsely Fractured Reservoir, Gas-Oil Gravity Drainage}

The second comparison between the dual permeability and DFM approaches involves gas-oil gravity drainage in a synthetic 3D reservoir. Viewed in a horizontal plane, the fracture pattern is the same as previous example (Figure 6.20a) but fractures are extended vertically from the top to the bottom of the reservoir (Figure 6.25). A horizontal producing well is completed at the bottom of the reservoir where it intersects several fractures. The petrophysical and fluid properties are presented in Table 6.4, indicating that the bottom layer has high matrix permeability. The horizontal producer is completed in this high-permeability layer to provide productivity during depletion. Table 6.4 also describes the initial conditions and wellbore operating constraints of this example. Production initially occurs due to fluid expansion. When the reservoir pressure drops below the bubble point pressure, 3664 psi, a two-phase oil-gas system develops and gravity drainage occurs as a result of the large oil-gas density difference and the highlypermeable vertical fractures.

We performed DFM and dual permeability simulations for this reservoir model. The spatial distribution of pressure after 5 years of production simulated by USDFM, EDFM, and dual permeability results in pressure maps that are reasonably similar (Figure 6.26). Furthermore, Figure 6.26 illustrates the impact of the changing production mechanism. The producing well creates a pressure gradient in the fractures connected to it, which in turn create a pressure drawdown on the adjacent matrix. Thus, fractures connected to the producing well play an important role in bringing oil to the producer. Figure 6.26 also shows that the pressure in parts of the reservoir is below the bubble point pressure at the illustrated time-step, suggesting the presence of both oil and gas. This is confirmed by Figure 6.27, which shows the spatial distribution of oil saturation at the same time-step as in Figure 6.26. Although small differences are observed, a reasonable 
agreement is evident between the dual permeability and DFM results. Figure 6.27a also shows oil saturation in the fractures, indicating that fractures are nearly saturated with gas. Figure 6.28 compares the oil-production rate and cumulative oil produced predicted by the three models. The ultimate oil recovery calculated by the dual permeability model is underestimated by $6 \%$ compared to the nearly identical forecasts of the DFMs. The differences in oil recovery curves start when the reservoir pressure drops below the bubble point pressure. However, the differences present in Figures 6.26, 6.27, and 6.28 are relatively small, thus affirming our earlier observation that the three modeling methods tested here are in close agreement for a sparsely fractured reservoir.

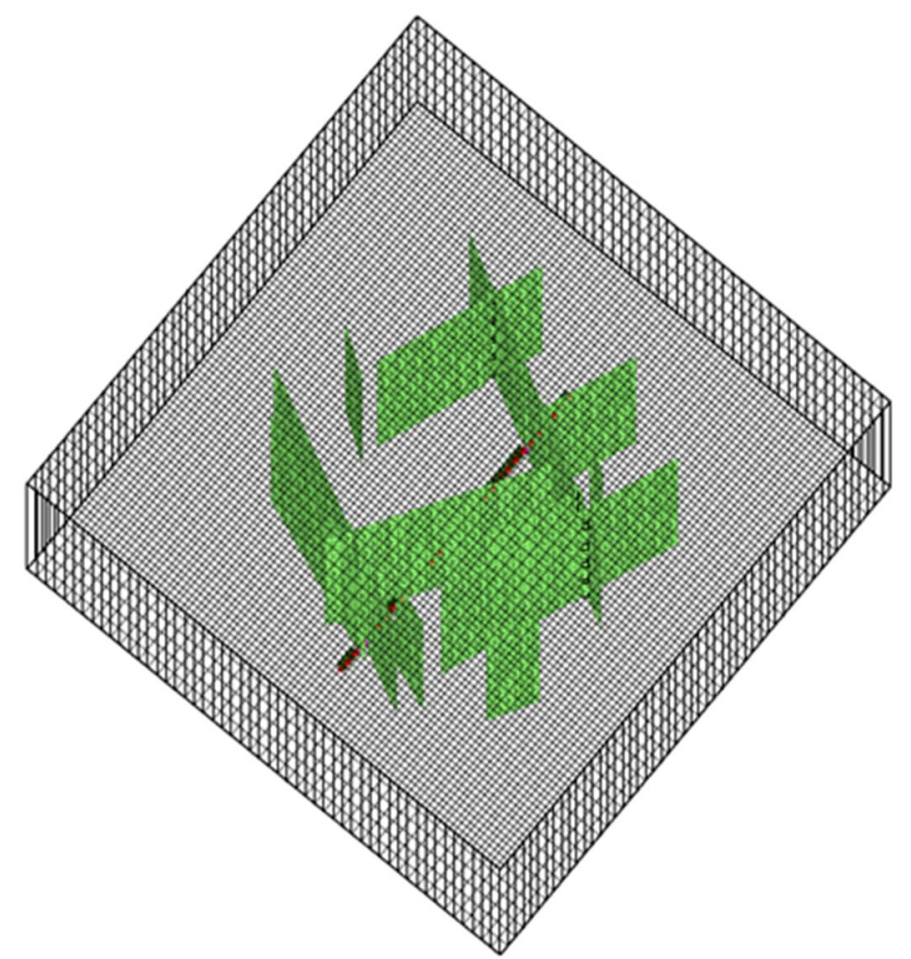

Figure 6.25: A 3D $(5100 \mathrm{ft} \times 5100 \mathrm{ft} \times 2000 \mathrm{ft})$ sparsely fractured reservoir studied in the second comparison case. A horizontal producer is completed at the bottom of reservoir in a high-permeability layer to provide productivity during depletion. The horizontal producer intersects some discrete fractures. 


\begin{tabular}{|c|c|c|c|}
\hline Variable & Value & Variable & Value \\
\hline Matrix porosity & 0.03 & Bottom-layer porosity & 0.05 \\
\hline Matrix permeability & $1 \mathrm{md}$ & Bottom-layer perm. & $1000 \mathrm{md}$ \\
\hline Fracture aperture & $0.5 \mathrm{~mm}$ & Reservoir pressure & $10000 \mathrm{psi}$ \\
\hline Fracture permeability & $2.11 \times 10^{7} \mathrm{md}$ & Reservoir temperature & $228^{\circ} \mathrm{F}$ \\
\hline Initial oil saturation & 1.0 & Producer BHP & $1000 \mathrm{psi}$ \\
\hline
\end{tabular}

Oil-gas relative permeability and capillary pressure data for the matrix

\begin{tabular}{|c|c|c|c|}
\hline $\boldsymbol{S}_{\boldsymbol{g}}$ & $\boldsymbol{k}_{\boldsymbol{r g}}$ & $\boldsymbol{k}_{\boldsymbol{r} \boldsymbol{}}$ & $\boldsymbol{P}_{\boldsymbol{c o g}}, \mathrm{psi}$ \\
\hline 0.050 & 0 & 1 & 1.283 \\
\hline 0.084 & 0 & 0.991 & 1.603 \\
\hline 0.119 & 0 & 0.970 & 1.924 \\
\hline 0.153 & 0 & 0.922 & 2.484 \\
\hline 0.188 & 0.001 & 0.830 & 2.938 \\
\hline 0.223 & 0.002 & 0.693 & 3.312 \\
\hline 0.257 & 0.004 & 0.539 & 3.653 \\
\hline 0.292 & 0.008 & 0.400 & 3.943 \\
\hline 0.326 & 0.012 & 0.290 & 4.171 \\
\hline 0.361 & 0.017 & 0.208 & 4.371 \\
\hline 0.396 & 0.024 & 0.148 & 4.639 \\
\hline 0.430 & 0.033 & 0.105 & 5.003 \\
\hline 0.465 & 0.043 & 0.074 & 5.245 \\
\hline 0.500 & 0.056 & 0.051 & 5.420 \\
\hline 0.534 & 0.071 & 0.035 & 5.753 \\
\hline 0.569 & 0.088 & 0.024 & 6.108 \\
\hline 0.603 & 0.107 & 0.015 & 6.326 \\
\hline 0.638 & 0.129 & 0.009 & 6.663 \\
\hline 0.673 & 0.155 & 0.005 & 7.088 \\
\hline 0.707 & 0.183 & 0.002 & 7.629 \\
\hline 0.742 & 0.214 & 0 & 8.429 \\
\hline 0.850 & 1 & 0 & 12.344 \\
\hline & & & \\
\hline & & 0 & \\
\hline
\end{tabular}

Table 6.4: Petrophysical properties assumed in the simulations performed for the model reservoir shown in Figure 6.25. 


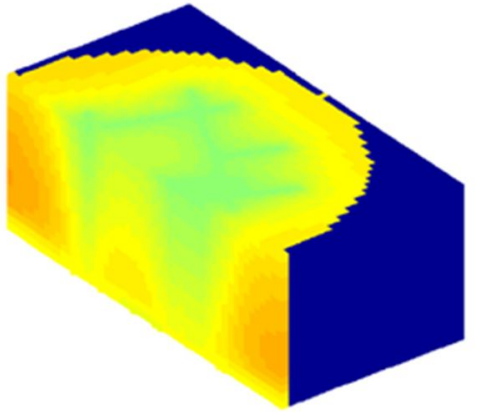

(a)

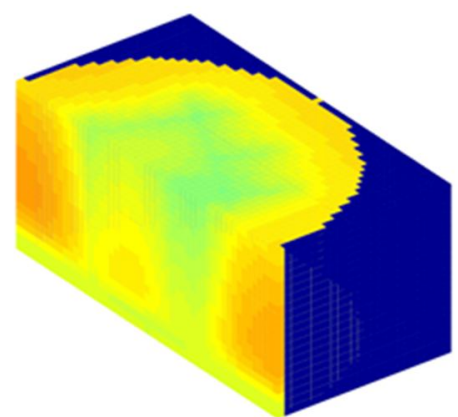

(b)

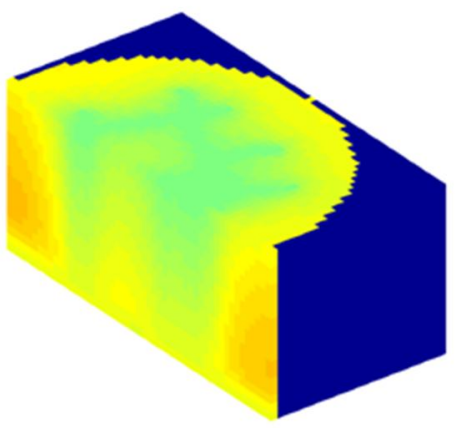

(c)

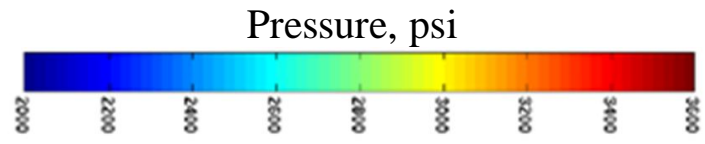

Figure 6.26: Cut-section view of profiles of pressure after 5 years of production simulated by (a) USDFM, (b) EDFM, and (c) dual permeability models for the fractured reservoir shown in Figure 6.25.

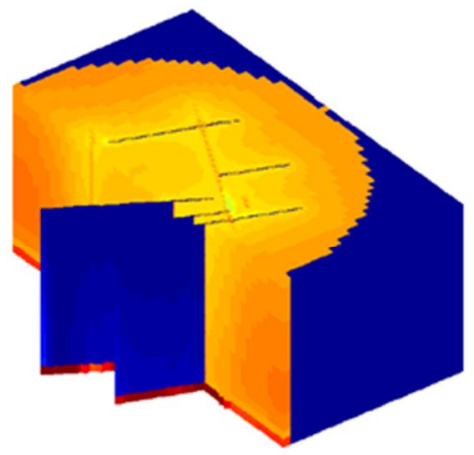

(a)

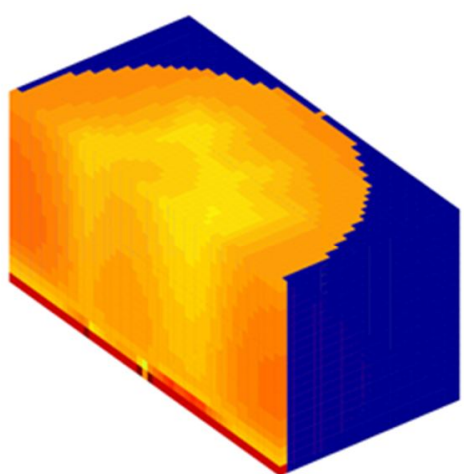

(b)

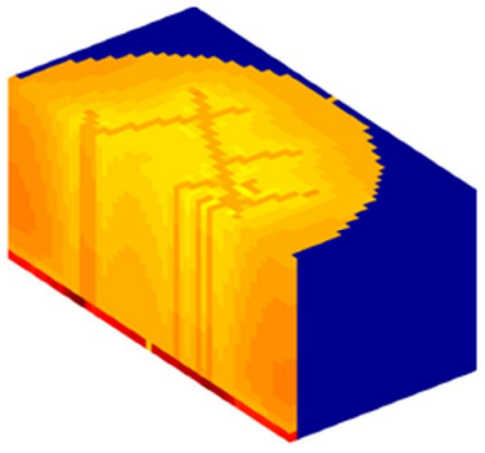

(c)

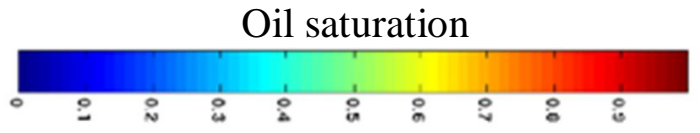

Figure 6.27: Cut-section view of profiles of oil saturation after 5 years of production simulated by (a) USDFM, (b) EDFM, and (c) dual permeability models for the fractured reservoir shown in Figure 6.25. Panel (a) also shows the oil saturation in some fractures. 


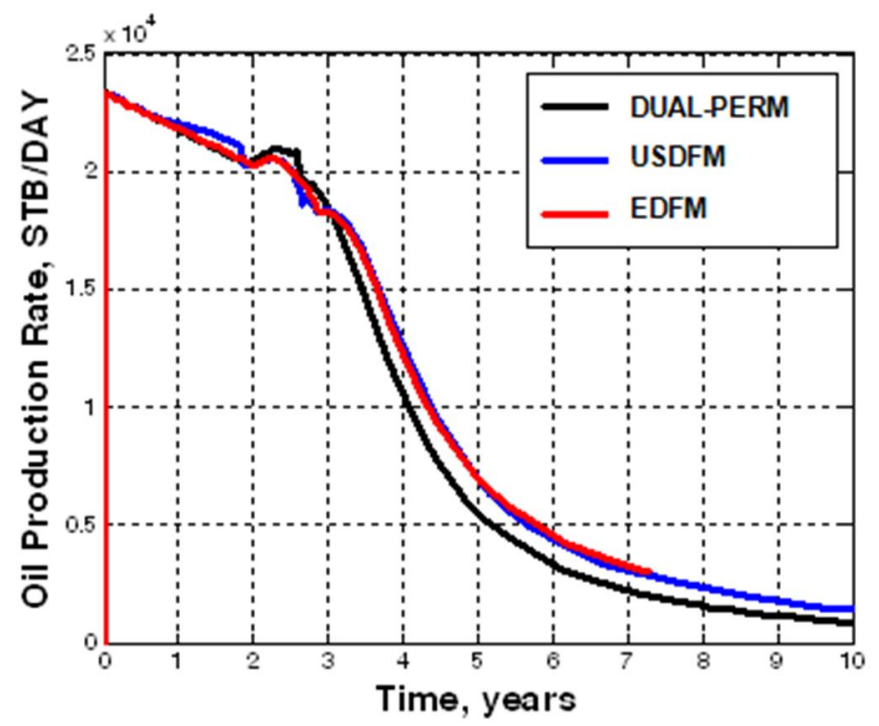

(a)

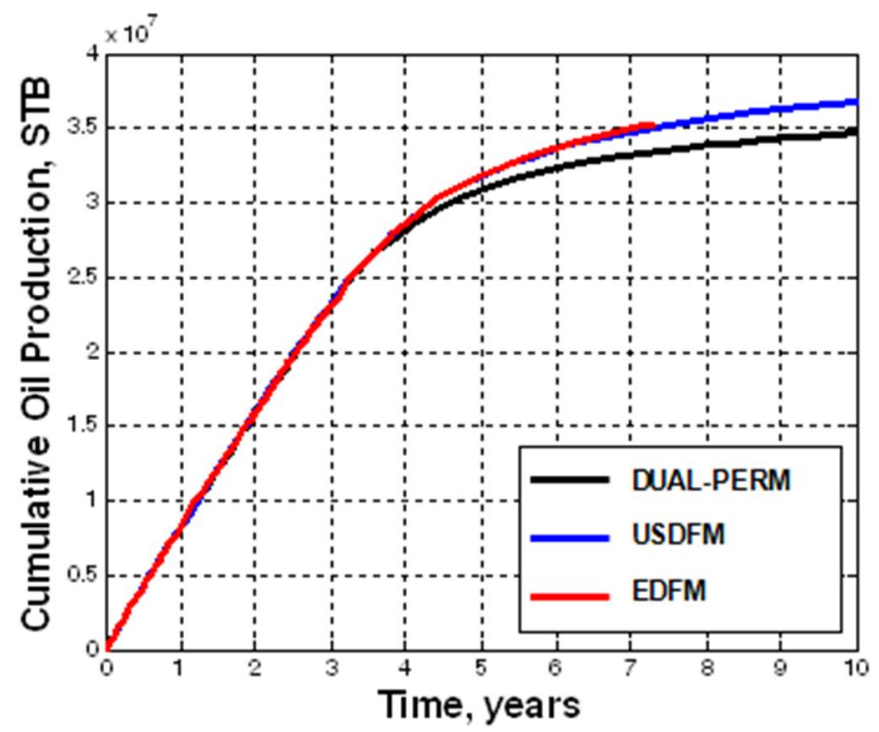

(b)

Figure 6.28: Comparison of (a) oil production-rate and (b) cumulative oil produced, calculated by USDFM, EDFM, and dual permeability models for the fractured reservoir shown in Figure 6.25. 


\subsubsection{Irregular and Anisotropic 2D Fractured Reservoir, Water Injection}

The synthetic 2D reservoir model in Figure 6.29 has 254 fractures arranged as two sets of aligned fractures with heterogeneous distributions. One set strikes approximately $030^{\circ}$ azimuth and the other strikes approximately $120^{\circ}$. The intensity of fractures varies in different regions. For instance, fractures oriented at $120^{\circ}$ are most abundant in the southwest portion of the model, and rare in the north. A few fractures are near injecting/producing wells, affecting fluid flow significantly. All petrophysical parameters and fluid properties, except the injector operating constraint, remain identical to those of the first comparison example. In this example, the injector is injecting water at constant BHP of 12000 psi.

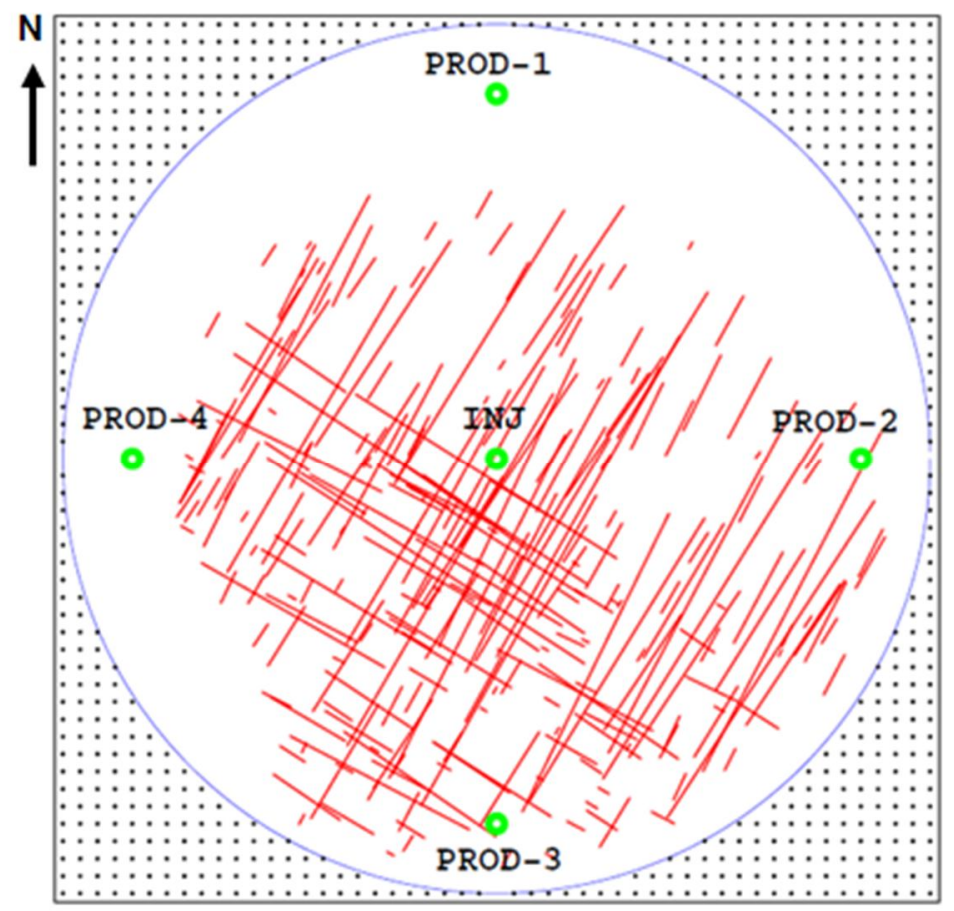

Figure 6.29: A synthetic $2 \mathrm{D}(5100 \mathrm{ft} \times 5100 \mathrm{ft})$ complex fractured reservoir studied in the third comparison example. This model comprises 254 fractures in two nearly orthogonal sets. The reservoir thickness is $20 \mathrm{ft}$. 
Here we are interested to assess the effect of the fracture network in oil recovery for a fractured reservoir with a higher degree of geometrical complexity. Figure 6.30 compares the field water-cut prediction from a matrix-only model with one in which fractures are included, using the USDFM approach. As expected, water breakthrough time is significantly earlier in the presence of highly fluid-conductive fractures. Producer 3 records the earliest water breakthrough time among producing wells while the latest one occurs at the Producer 1.

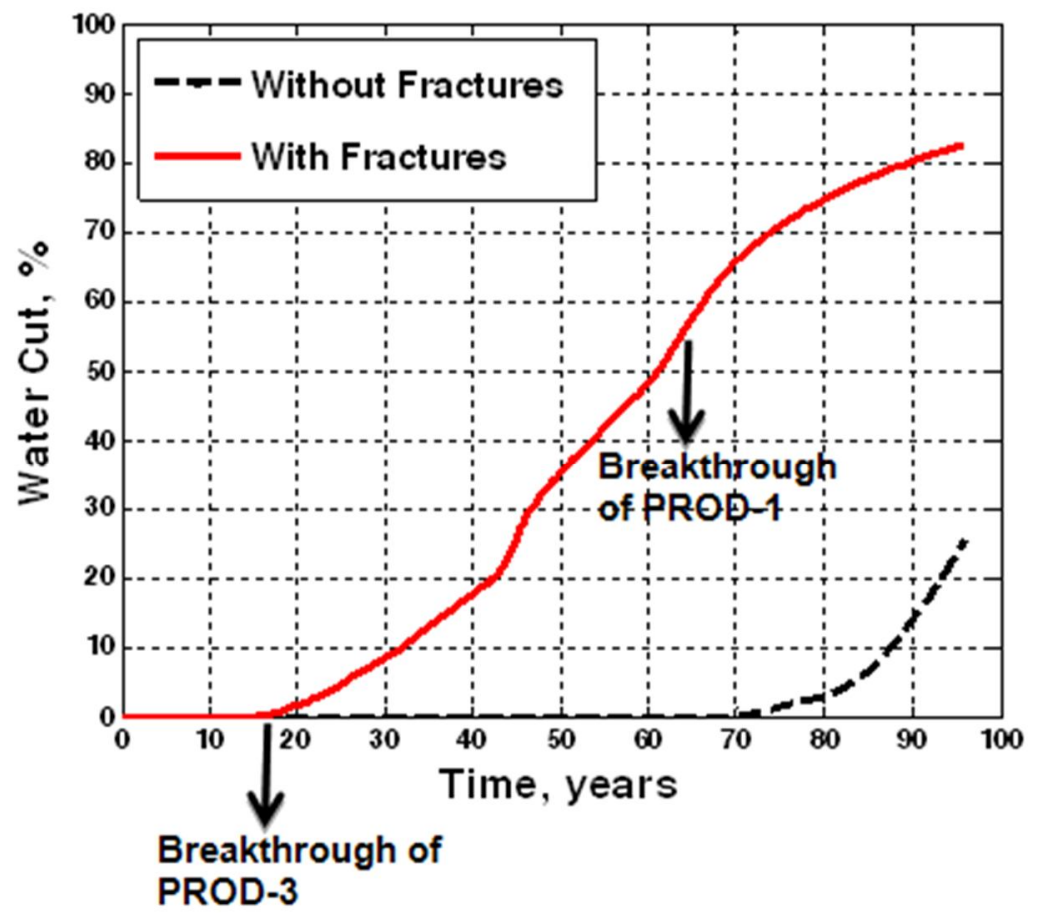

Figure 6.30: Comparison of water-cut calculated by USDFM approach in the presence and absence of fractures. In the presence of fractures, Producer 3 records the earliest water breakthrough time among producing wells while the latest breakthrough occurs at Producer 1. 
We performed simulations with both DFMs and dual permeability for this complex NFR. For this case, the dual permeability results are remarkably different from those of the DFM simulations. Figure 6.31 shows the water saturation maps after 1616 and 5356 days of water injection simulated by DFMs and the dual permeability model. Similar to previous examples, good agreement exists between the results of USDFM and EDFM approaches. The dual permeability model, however, significantly smears the water saturation distribution compared to the channelized flow evident in the DFM results. Figure 6.32 further shows that the water-cut calculated by dual permeability simulation is also considerably different from those predicted by the DFMs. For instance, water breakthrough time predicted by the dual permeability model occurs about 10 years later than those predicted by the DFMs.

It is clear from these results that the dual permeability model is not able to capture the strong anisotropy of flow caused by the irregular fractures that are not aligned with the grid. Relative to the size of the grid cells, the fracture variability is too complex to model accurately with a dual medium approach because the discrete nature of the fractures cannot be distinctly maintained. In contrast, the consistency between the results of the DFMs, which are based on entirely different technologies, suggests that their forecasts are reliable. 

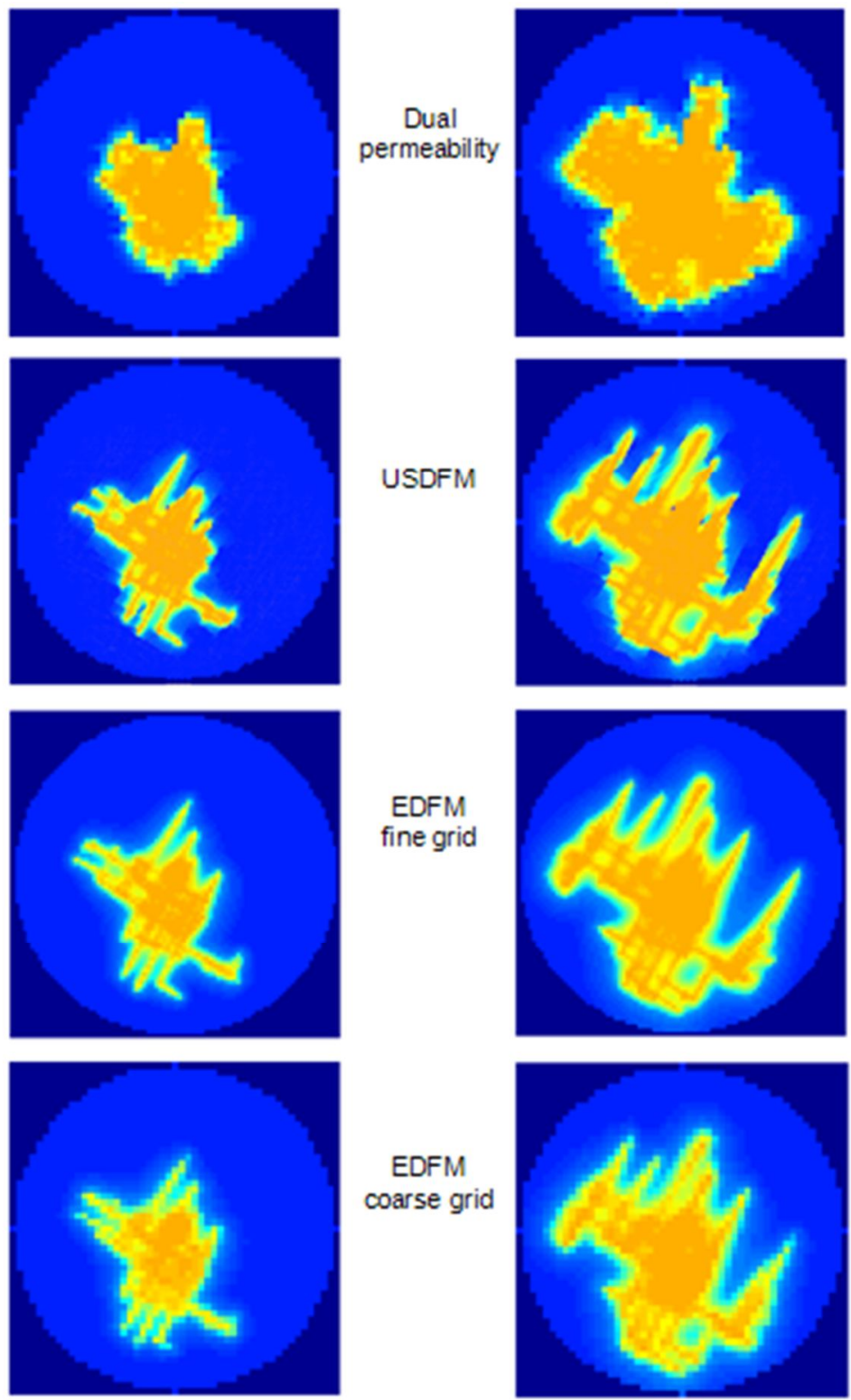

(a)

(b)

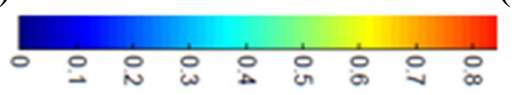

Water saturation

Figure 6.31: Profiles of water saturation after (a) 1616 and (b) 5356 days of water injection acquired in the fractured reservoir shown in Figure 6.29. The first, second, third, and forth rows correspond to simulations of dual permeability, USDFM, fine-grid $(85 \times 85)$ EDFM, and coarse-grid $(51 \times 51)$ EDFM approaches, respectively. 


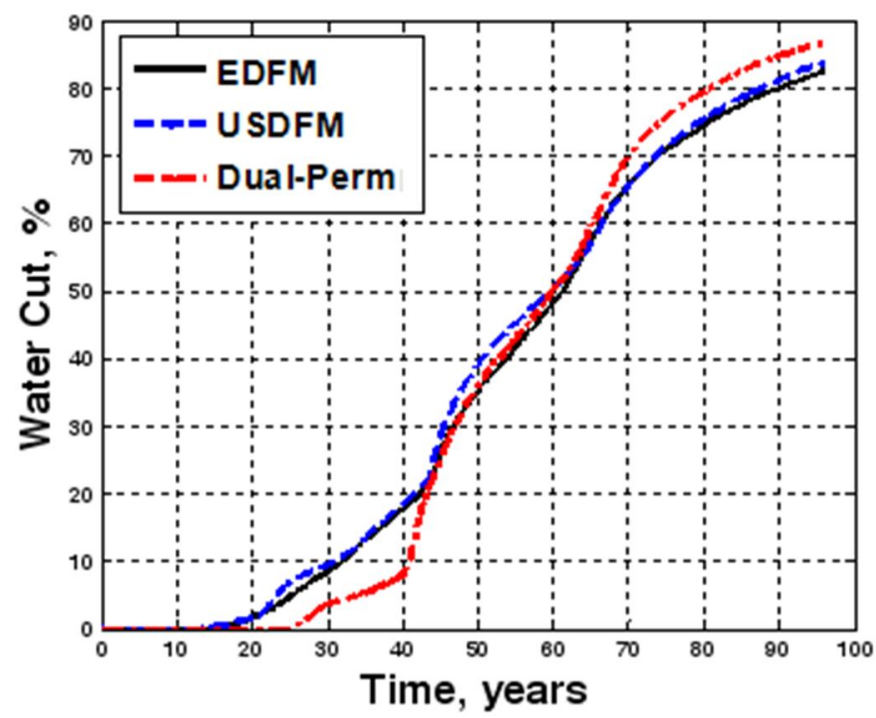

(a)

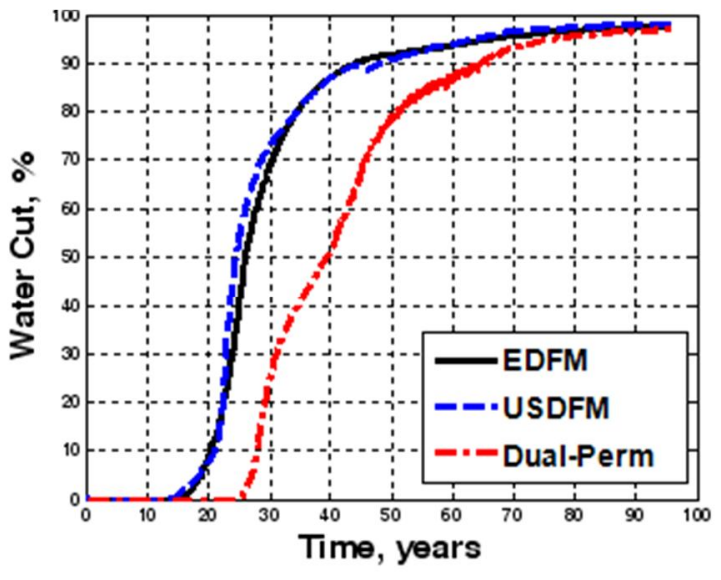

(b)

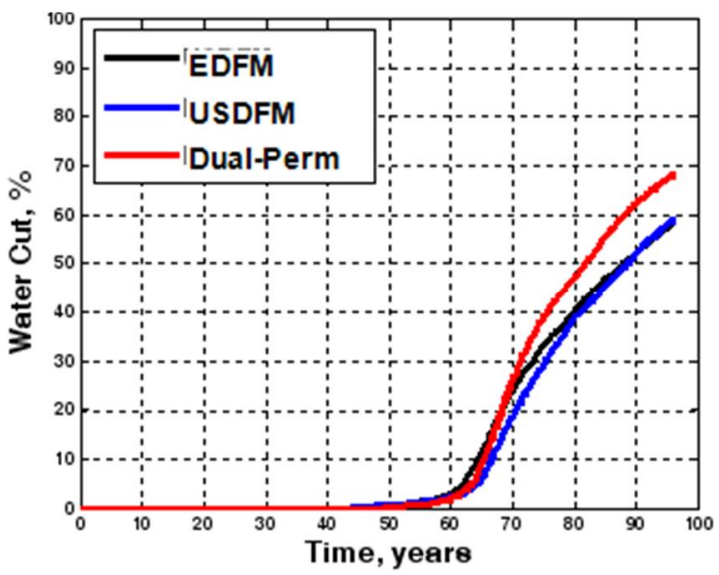

(c)

Figure 6.32: Comparison of (a) field water-cut, (b) water-cut of Producer 3 which has the earliest breakthrough time, and (c) water-cut of Producer 1 which has the latest breakthrough time, calculated by EDFM, USDFM, and dual permeability models for the fractured reservoir shown in Figure 6.29. 
We also evaluated the impact of grid resolution by comparing the results from this complex model based on fine and coarse grids. The third row in Figure 6.31 shows results of an EDFM simulation performed on an $85 \times 85$ Cartesian grid. We repeated the EDFM simulation on a $51 \times 51$ Cartesian grid, and results are shown in the fourth row of Figure 6.31. The water saturation maps obtained with the coarse-grid simulation are qualitatively similar to those obtained with the fine-grid simulation. The water-cut curves for these simulations shown in Figure 6.33 are very similar, but show water breakthrough occurring earlier in the fine-grid model.

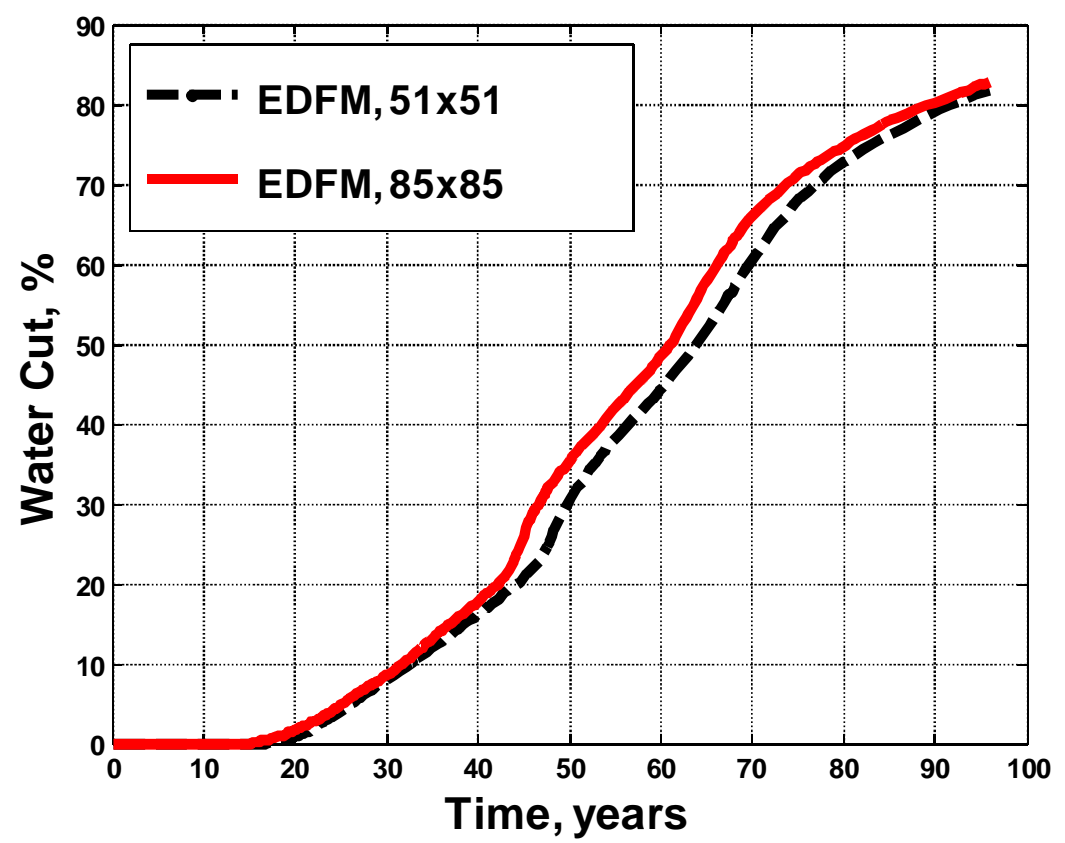

Figure 6.33: Comparison of field water-cut, calculated by fine-grid $(85 \times 85)$ and coarsegrid $(51 \times 51)$ EDFM simulations for the fractured reservoir shown in Figure 6.29 . 


\subsubsection{Discussion of DFMs}

The EDFM method is fundamentally a simpler approach than USDFM as it does not require specialized gridding. With the same background grid resolution, EDFM simulations are usually faster than USDFM simulations because the local refinement of the unstructured grid leads to a larger number of grid cells. EDFM is conceptually similar to dual continuum approaches, but improves them in several ways. Fluid transport between matrix and each individual fracture is systematically calculated based on the fracture geometry, rather than general transfer functions which rely on effective parameters for fracture spacing. Embedded fractures are able to transport fluids in any direction relative to the grid axes which makes it possible to construct models that reflect the high localized anisotropy observed in naturally fractured reservoirs. The USDFM technique requires specialized gridding and hence substantial modifications from conventional simulation workflows that are based on structured grids. However, Hui et al. (2008) and Lim et al. (2009) showed that multiple grids can be constructed for simulation during the course of practical studies.

Comparing the computational performance of the two DFM methods was not straightforward because they are designed to run with simulators that have different solvers and native grid structures. The EDFM was run on a proprietary general-purpose simulator that was designed for structured grid models. The USDFM runs were performed using a next-generation simulator that was designed for parallel simulation of unstructured grid models (DeBaun et al., 2005). Because performance data are not directly comparable we do not present them here. 


\subsubsection{Concluding Remarks}

Simulations showed that the EDFM approach is insensitive to grid orientation. Simulations also showed consistency and agreement of results using the EDFM and USDFM methods in synthetic models with complex fracture patterns, in contrast with the less-satisfactory results from a conventional dual permeability model. Although dual continuum approaches were originally introduced for studying the behavior of densely fractured reservoirs with good connectivity, we found that the dual permeability approach described in this study is reasonably accurate for predicting the behavior of sparsely fractured systems. The simulations showed that for very sparse fractured reservoirs, where fracture spacing is much larger than grid cell size, the dual permeability model can capture the general trends. However, when the fracture density is greater and fracture pattern is more irregular, our tests showed that the dual permeability model is generally unable to resolve details of complex flow paths and high, localized anisotropy in naturally fractured reservoirs. In contrast, both EDFM and USDFM were found to be capable of representing flow anisotropy and preferential channeling caused by realistic fracture geometries. 


\subsection{Highlights OF THE CHAPTer}

- Examples of water-flooding, compositional gas injection, and primary depletion are presented to demonstrate the applicability, robustness, and efficiency of the EDFM approach (developed in GPAS) for modeling fluid flow in naturally fractured reservoirs.

- The grid sensitivity study, once again, verifies that the EDFM approach can achieve high accuracy using moderate mesh refinement.

- The EDFM approach is found to be insensitive to grid orientation.

- Simulations show consistency and agreement of results using the EDFM approach with results using an unstructured discrete fracture model (USDFM) for synthetic models with complex fracture patterns.

- Simulations also show that the dual permeability model fails to provide accurate solutions in the presence of large-scale fractures and high localized anisotropy. 


\section{Chapter 7: Incorporation of Dynamic Behavior of Fractures into EDFM}

One advantage of the embedded discrete fracture model (EDFM) developed in this research is that it can be easily extended to many applications by incorporating different physics in the model. For instance, a number of studies have demonstrated the dynamic behavior of fractures with pressure changes and the subsequent effects on hydrocarbon recovery. Hence, we have incorporated the dynamic behavior of fractures into EDFM. In this chapter, we present the methodology used to implement fracture deformation into EDFM, followed by several example simulations.

\subsection{INTRODUCTION}

Pore pressure changes caused by production from a reservoir or injection into a reservoir can induce rock deformations. Fluid-flow characteristics of reservoir rocks, such as permeability and pore compressibility, can be very sensitive to effective stress changes caused by changes in pore pressure. Settari and Mourits (1998) coupled a commercial reservoir simulator with a three-dimensional geomechanics code to account for interactions between geomechanics and multiphase flow. Chin et al. (2000) developed a fully coupled geomechanics and fluid-flow model to analyze pressure transient problems in stress-sensitive reservoirs with nonlinear elastic and plastic constitutive behavior. Also, Raghavan and Chin (2004) presented correlations to evaluate productivity losses owing to permeability changes in stress-sensitive reservoirs. Gutierrez et al. (2001) discussed the issues related to the interaction between rock deformation and fluid-flow 
behavior of hydrocarbon reservoirs. Moreover, Samier et al. (2006) discussed and compared various approaches to perform coupled reservoir-geomechanical simulations.

The effect of geomechanics on fluid flow is more crucial in fractured media due to presence of fissures, which might be more stress-sensitive than the rock matrix. Fractures are the major conduits for fluid flow in fractured reservoirs and thus, fracture permeability plays an important role in production. The flow characteristics of fractures are significantly affected by effective normal stress acting on them. Bandis et al. (1983) and Barton et al. (1985) presented laboratory experiments on fractured cores to propose an unfilled joint deformation model. Also, experiments conducted by Wilbur and Amedei (1990) and Makurat and Gutierrez (1996) showed that the transmissivity of a fracture depends on the stress acting on that fracture. Likewise, Lorenz (1999) discussed stress sensitivity of fractures in several reservoirs wherein variations in reservoir deliverability during production are caused by changes in permeability of the fractured system. Moreover, Pinzon et al. (2000) presented field data indicating that the fracture permeability is dynamic rather than static and declines with pressure depletion. In spite of extensive experimental and field studies that have demonstrated the dynamic behavior of fractures, flow characteristics of fractures have been often treated as static parameters in simulations of naturally fractured reservoirs. Hence, changes in fracture aperture, porosity, and permeability with pressure changes should be accounted for in modeling fractured systems.

A coupled fluid-flow and geomechanics model is required for a better understanding and accurate modeling of naturally fractured reservoirs. Bai et al. (1999) are among the first who considered the effect of fracture deformation on permeability in flow simulations but they neglected the non-linearity of fracture deformation. Later, Pao and Lewis (2002), Shchipanov and Nazarov (2005), Zhao and Chen (2006), Shchipanov 
and Rusakov (2008), Khalili (2008), Bagheri and Settari (2008), Tao et al. (2009), and Ranjbar et al. (2011) incorporated fracture geomechanics into modeling of naturally fractured reservoirs. For example, Bagheri and Settari (2008) included the effect of fractured-media deformation into a dual porosity approach by incorporating dynamic tensor permeability for fractures. None of the above-mentioned studies considered discrete fracture network modeling. There have been very few attempts to couple flow and geomechanics in discrete fractures models. An attempt was made by Monteagudo et al. (2011) who coupled a finite-element poroelastic code with a control volume discrete fracture flow simulator.

We have incorporated the dynamic behavior of fractures into EDFM. The model allows inclusion of the impact of stress regime on fluid flow in a $3 \mathrm{D}$ discrete fracture network. We use empirical joint models to represent normal deformation of pre-existing natural fractures and couple them with the EDFM approach. Using these models, the aperture and permeability of an arbitrarily-oriented fracture become functions of the effective normal stress acting on the fracture plane. In addition, we allow for fractureconductivity tables to model dynamic behavior of propped hydraulic fractures in stimulated reservoirs.

In the following sections, we first describe empirical joint models that relate deformation of fractures to effective stress changes. We also describe the methodology used to couple the discrete fracture model with fracture deformation. Several examples are then presented to demonstrate the dynamic behavior of fractures and its impact on hydrocarbon production in naturally fractured reservoirs. We illustrate the dependency of fracture aperture and permeability on pressure changes during simulations. Furthermore, we examine the dynamic behavior of propped hydraulic fractures in unconventional gas reservoirs. 


\subsection{FraCtURE DEFORMATION}

Fully-coupled geomechanics and flow models can provide an accurate environment to incorporate geomechanical effects into fluid-flow simulation of fractured reservoirs. In these models, mechanical deformation of rock and fractures is numerically simulated. However, fully-coupled approaches for modeling fractured systems are numerically difficult and complex to implement and computationally expensive. In order to include dynamic behavior of fractures in the simulation of fractured reservoirs, one simplified approach is to use stress- or pressure-dependent empirical models for fracture deformation. In this section, first, we present empirical models that describe dynamic behavior of natural fractures with pressure changes. Then, we describe pressuredependent conductivity of propped hydraulic fractures.

\subsubsection{Deformation of Natural Fractures}

Pore pressure depletion due to hydrocarbon production induces more compression on fracture planes, thereby reducing the fracture aperture. Goodman (1974) explained the basic mechanics of joint normal deformation and performed experiments to show that the joint closure under increasing normal stress varies in a non-linear manner resembling a hyperbola. Later, Bandis et al. (1983) and Barton et al. (1985) used a large number of experimental data on interlocked block samples with natural unfilled joints. They conducted experiments on fresh and weathered joint samples from five rock types, namely slate, dolerite, limestone, siltstone, and sandstone. The interlocked joint samples were subjected to a sequence of loading/unloading cycles. Figure 7.1 shows typical normal stress vs. closure curves calculated from the experiments. 


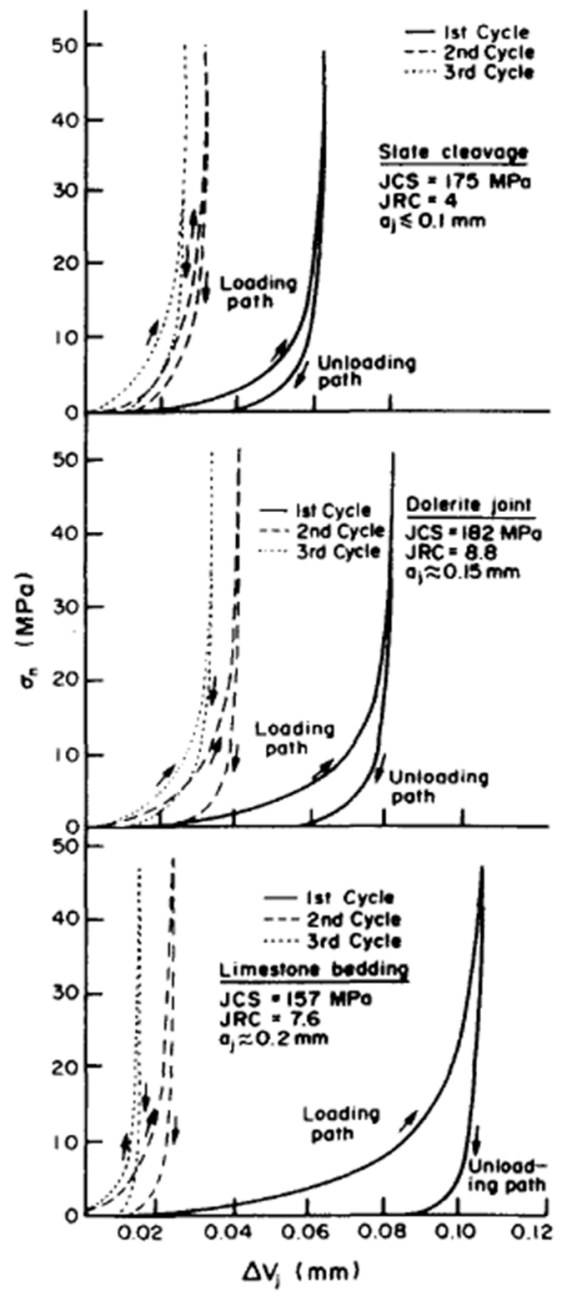

(a)

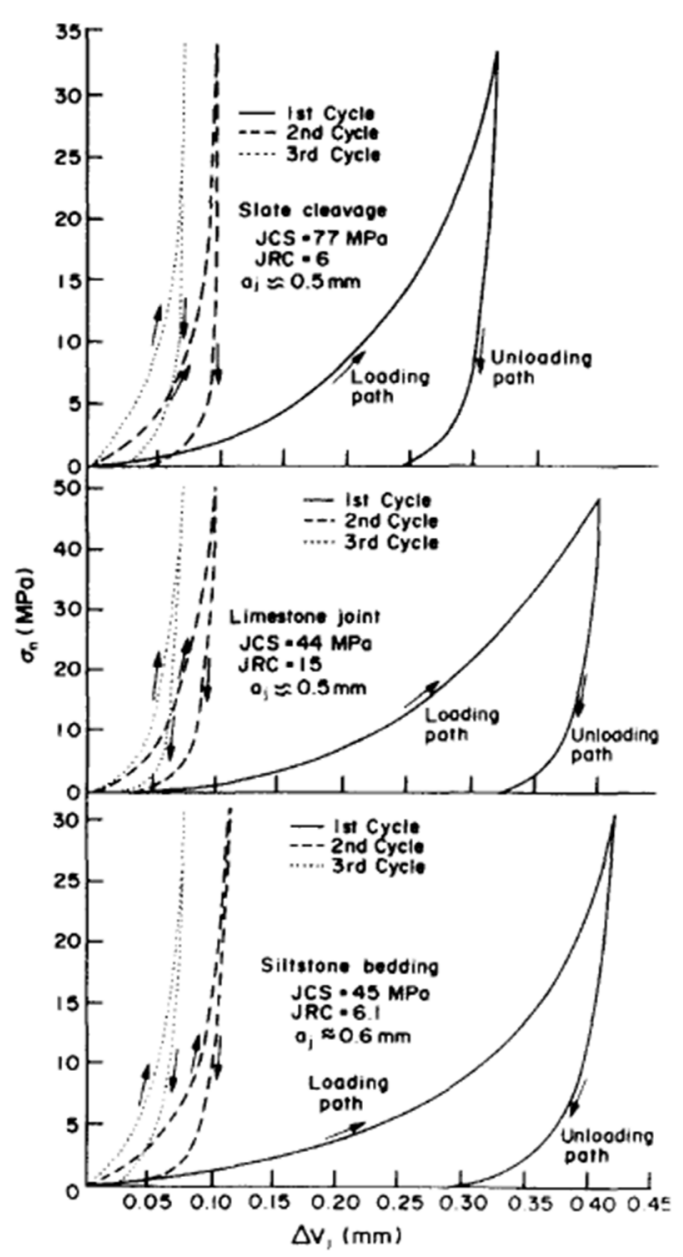

(b)

Figure 7.1: Normal stress $\left(\sigma_{n}\right)$ vs. closure $\left(\Delta V_{j}\right)$ curves for a range of (a) fresh joints and (b) weathered joints in different rock types, under repeated loading cycles (from Bandis et al., 1983). 
Based on their experimental data, Bandis et al. (1983) and Barton et al. (1985) suggested a hyperbolic model for the normal deformation of fractures as given below:

$$
\sigma_{n}=\frac{D_{n}}{a-b D_{n}}
$$

where $\sigma_{n}$ is the effective normal stress and $D_{n}$ is the normal closure of fracture. Also, $a$ and $b$ are constants related to two joint parameters, initial normal stiffness and maximum joint closure. For very large values of normal stress, the fracture closure reaches its maximum and thus,

$$
D_{n \max }=a / b
$$

where $D_{n \max }$ is the maximum normal closure of fracture. Also, joint normal stiffness $\left(K_{n}\right)$ is defined as the ratio of change in normal stress to change in normal closure and can be obtained from the derivative of Equation 7.1 as

$$
K_{n}=\frac{d \sigma_{n}}{d D_{n}}=\frac{1}{a\left(1-\frac{b}{a} D_{n}\right)^{2}}
$$

The normal stiffness at zero normal stress is called the initial normal stiffness $\left(K_{n i}\right)$, which corresponds to zero normal closure and can be written as

$$
K_{n i}=1 / a
$$

Hence, two basic joint parameters, initial normal stiffness and maximum normal closure, uniquely define the hyperbolic relationship between normal stress and closure of a fracture. These parameters can be determined experimentally. Thus, we can calculate the normal fracture closure in terms of joint parameters and normal effective stress (using Equations 7.1, 7.2, and 7.4), which is given by 


$$
D_{n}=\frac{D_{n \max } \sigma_{n}}{K_{n i} D_{n \max }+\sigma_{n}} .
$$

Normal stress $\left(S_{n}\right)$ acting on a fracture plane can be calculated by considering the orientation of the fracture with respect to the in-situ stress field. If principal in-situ stresses act in the $\mathrm{x}, \mathrm{y}$, and $\mathrm{z}$ directions, then the normal stress acting on an arbitraryoriented fracture plane is resolved by

$$
S_{n}=n_{x}^{2} S_{x x}+n_{y}^{2} S_{y y}+n_{z}^{2} S_{z z},
$$

where $n_{x}, n_{y}$, and $n_{z}$ are the directional cosines of the angles between the normal of the fracture plane and the principal stress axes, and $S_{x x}, S_{y y}$, and $S_{z z}$ are the present-day principal stresses acting in the $\mathrm{x}, \mathrm{y}$, and $\mathrm{z}$ directions, respectively. Subsequently, effective normal stress can be calculated by

$$
\sigma_{n}=S_{n}-P_{p},
$$

where $P_{p}$ is the pore pressure. Hence, in anisotropic stress field, different fracture planes experience different normal deformation depending on their orientations. The BartonBandis model is the most commonly applied deformation model for natural fractures in reservoir conditions. Other empirical models also exist which relate fracture normal closure to effective normal stress. For example, Evans et al. (1992) suggested a logarithmic relationship for fracture normal deformation but this model cannot be used at highly stressed in-situ conditions. Compared to normal deformation, fracture deformation behavior under shear stress is more complicated and requires a complex mathematical modeling. Furthermore, the effect of shear stress on fracture flow properties is secondary. Thus, we only consider the normal deformation of fractures in this study. 
In order to incorporate the dynamic behavior of fractures into EDFM, one simplified approach is to use stress- or pressure-dependent empirical models for fracture deformation rather than fully coupled fluid-flow and geomechanics models, which are more complicated and computationally expensive. Therefore, we use the non-linear Barton-Bandis joint model to represent deformation of natural fractures and couple it with the EDFM approach. As a result, fracture properties (aperture, porosity, and permeability) become functions of the effective normal stress acting on the fracture plane. At the end of each time-step in reservoir simulation, effective normal stress and normal closure are re-calculated for each fracture. Also, fracture permeability $\left(k_{f}\right)$, which is proportional to the fracture aperture square according to the Poiseuille law, is updated by

$$
k_{f}=k_{f i}\left(\frac{\omega_{f}}{\omega_{f i}}\right)^{2} \text {, }
$$

where $\omega_{f}$ is the new fracture aperture and subscript $i$ denotes the properties at the initial conditions. For the EDFM approach, as described in Chapter 4, we compute the transmissibility between fracture control volumes in the fracture domain, the transmissibility between matrix grids and fracture control volumes (connected through non-neighboring connections), the transmissibility between intersecting fractures (also connected through non-neighboring connections), and the transmissibility between fractures and wells using a pre-processing code prior to reservoir simulation. Hence, at the end of each time-step we re-calculate all these transmissibilities using the updated values of fracture apertures and fracture permeabilities. The new porosities, permeabilities, and transmissibilities are then returned to the flow equations for the next time-step. 


\subsubsection{Deformation of Propped Hydraulic Fractures}

Apart from naturally fractured reservoirs, development of unconventional resources through hydraulic fracturing of horizontal wells has received increased attention in the last few years. Hydraulic fracture conductivity is a key parameter to economic development of low-permeability reservoirs. However, the conductivity of hydraulic fractures is significantly reduced by pressure depletion. Fracture-deformation empirical models, previously mentioned, are appropriate for describing dynamic behavior of pre-existing natural fractures in in-situ conditions and are not necessarily applied to propped hydraulic fractures.

Some effort has been made to investigate the effect of closure stress on hydraulic conductivity of proppant packs. Maloney et al. (1989) showed that the proppant packs may crush under stress leading to reduction in the fracture porosity. Likewise, Fredd et al. (2001) presented laboratory data showing the effect of closure stress on propped fracture conductivity for a rock with relatively high Young's modulus. Therefore, recent studies such as Rubin (2010) and Cipolla et al. (2010) used specific closure-stressconductivity tables in the simulation of unconventional reservoirs to account for the dynamic behavior of hydraulic fractures. Closure stress acting on the hydraulic fracture is defined as the horizontal stress perpendicular to the fracture minus the pressure inside the fracture. Hence, for dynamic treatment of fractures in the EDFM approach we allow for closure-stress-conductivity tables as well as empirical fracture closure models. 


\subsection{EXAMPLE SimUlationS}

In this section, we present several examples to investigate the effect of pressuredependent fracture aperture and permeability on production of fractured reservoirs. The first set of examples investigates the effect of fracture deformation on oil production from naturally fractured reservoirs using the Barton-Bandis empirical joint-deformation model. The second example compares gas production from a hydraulically-fractured lowpermeability reservoir under static and dynamic treatments of fractures.

\subsubsection{Depletion of an Oil Reservoir}

A synthetic 2D model reservoir shown in Figure 7.2 is evaluated in the first example, which is used as the base case. The model contains a few long, vertical, and highly-conductive fractures. Reservoir dimensions are $1000 \times 1000 \times 50 \mathrm{ft}$. A vertical well is located at the center of the model and intercepted by one long fracture. All fractures and the well completely penetrate the height of the formation. The reservoir fluid is oil with specific gravity of 0.74 and viscosity of $1.5 \mathrm{cp}$, and the formation porosity and permeability are 0.26 and $0.1 \mathrm{md}$, respectively. Also, the formation temperature and the

rock compressibility are $120^{\circ} \mathrm{F}$ and $5 \times 10^{-6} \mathrm{psi}^{-1}$, respectively. The water saturation in rock matrix is at the irreducible water saturation of 0.2 and the endpoint relative permeability of oil is 0.8 .

The initial reservoir pressure is $4000 \mathrm{psi}$ and the vertical well produces at a constant bottomhole pressure of $2000 \mathrm{psi}$, which is above the bubble point pressure. The vertical stress is 7500 psi while the minimum horizontal stress $\left(S_{\text {hmin }}\right)$ and maximum horizontal stress $\left(S_{H \max }\right)$ are 4500 psi and 7200 psi, respectively, representing high horizontal stress anisotropy. Fractures are oriented at different angles ranging from parallel-to- $S_{\text {hmin }}$ to parallel-to- $S_{H \max }$. Also, initial normal stiffness and maximum closure 
for all fractures are $7 \times 10^{4} \mathrm{psi} / \mathrm{ft}$ and $0.011 \mathrm{ft}$, respectively. Initial aperture of fractures are assigned using the Barton-Bandis model and assuming that the maximum aperture size is equal to the maximum closure and corresponds to zero effective normal stress. By rewriting Eq. 7.5, we can calculate the fracture aperture as

$$
\omega_{f}=D_{n \max } \frac{K_{n i} D_{n \max }}{K_{n i} D_{n \max }+\sigma_{n}} .
$$

Thus, fractures have different initial apertures, ranging from 0.65 to $2.03 \mathrm{~mm}$. Initial permeability of fractures, which is proportional to the square of initial fracture aperture, therefore ranges from 43 to 418 Darcies.

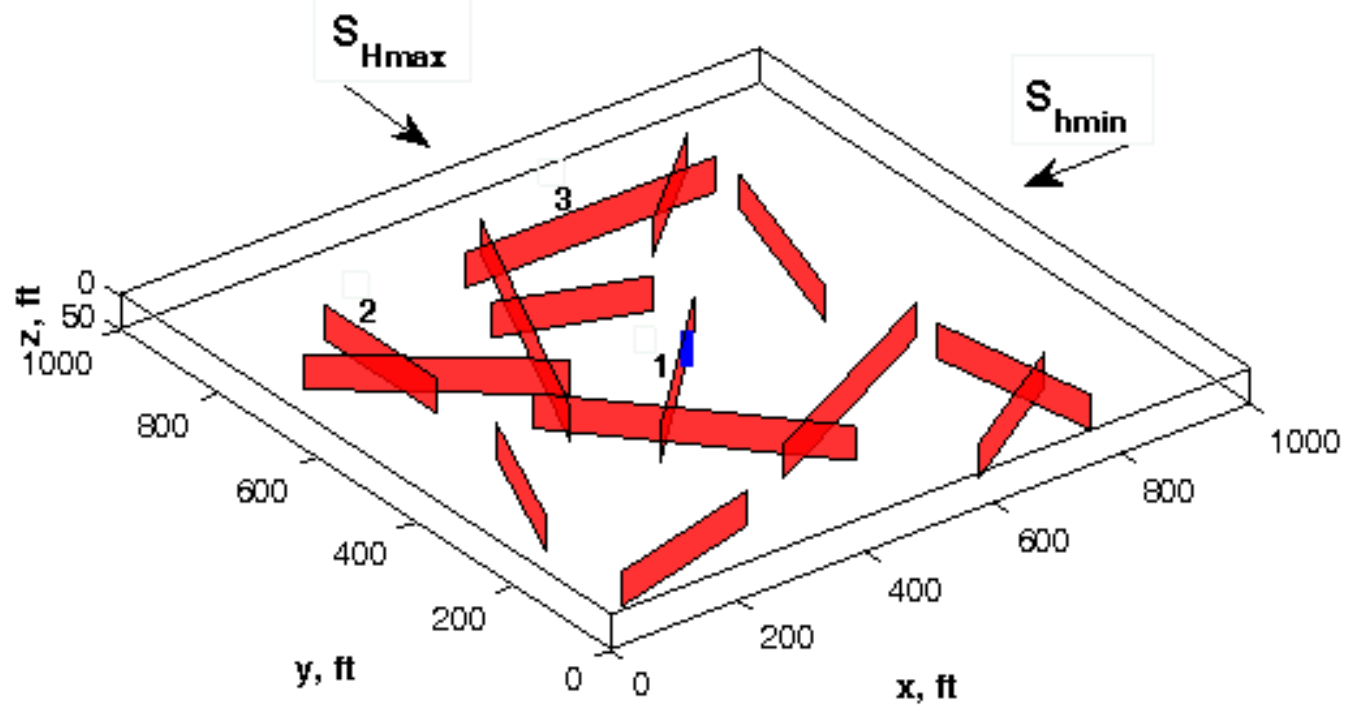

Figure 7.2: A synthetic $2 \mathrm{D}$ fractured reservoir $(1000 \times 1000 \times 50 \mathrm{ft})$ considered in the first example. Three fractures are shown with numbers for detailed investigation during production. 
The matrix grid is $45 \times 45 \times 1$ cells in $\mathrm{x}, \mathrm{y}$, and $\mathrm{z}$ directions, respectively, and there are 267 fracture control volumes in the fracture domain, representing 14 discrete fractures in the model. We investigate the behavior of three fractures, which are shown with numbers in Figure 7.2, in more detail. The first fracture is directly connected to the wellbore while the second and third fractures are oriented parallel to $S_{H \max }$ and $S_{\text {hmin }}$, respectively. Figure 7.3 shows the ratio of fracture aperture to the maximum closure (maximum aperture) for the given joint parameters, depicting the nonlinear relationship of effective normal stress and fracture closure. At the initial conditions, Fracture 2, which is perpendicular to $S_{h \min }$, is under the lowest effective normal stress (marked with a red circle in Figure 7.3) and has the largest initial aperture among fractures while Fracture 3, which is perpendicular to $S_{\text {Hmax }}$, is under the highest effective normal stress (marked with a blue square in Figure 7.3) and has the smallest initial aperture. This suggests that the fractures oriented normal to the minimum principal stress are the most conductive.

Since the pressure drawdown in this example is 2000 psi, pressure inside each fracture may decrease by 2000 psi during simulation. After pressure depletion of 2000 psi in the fractures, Fracture 2 would lose $61 \%$ of its initial aperture (as shown in Figure 7.3) and subsequently, lose $85 \%$ of its initial permeability. However, Fracture 3 would lose $33 \%$ of its initial aperture and $55 \%$ of its initial permeability. Therefore, fractures under lower initial effective normal stress are prone to larger aperture decline and permeability loss due to pressure depletion. That is, the rate of deformation is greatest at low values of normal stress.

Figure 7.4a shows the pressure histories simulated for a segment of the abovementioned fractures over two years of production. Correspondingly, Figure $7.4 \mathrm{~b}$ presents the permeability profiles of the fractures during simulation. Fracture 1, which is intercepted by the wellbore, experiences a rapid pressure drop over the first days of 
production, which results in significant reduction in its hydraulic conductivity. However, fractures 2 and 3 undergo moderate pressure depletion over the simulation duration. Figure $7.4 \mathrm{~b}$ indicates that the permeabilities of fractures 1,2 , and 3 are reduced to 0.38 , 0.25 , and 0.59 of their initial values, respectively, over two years of production.

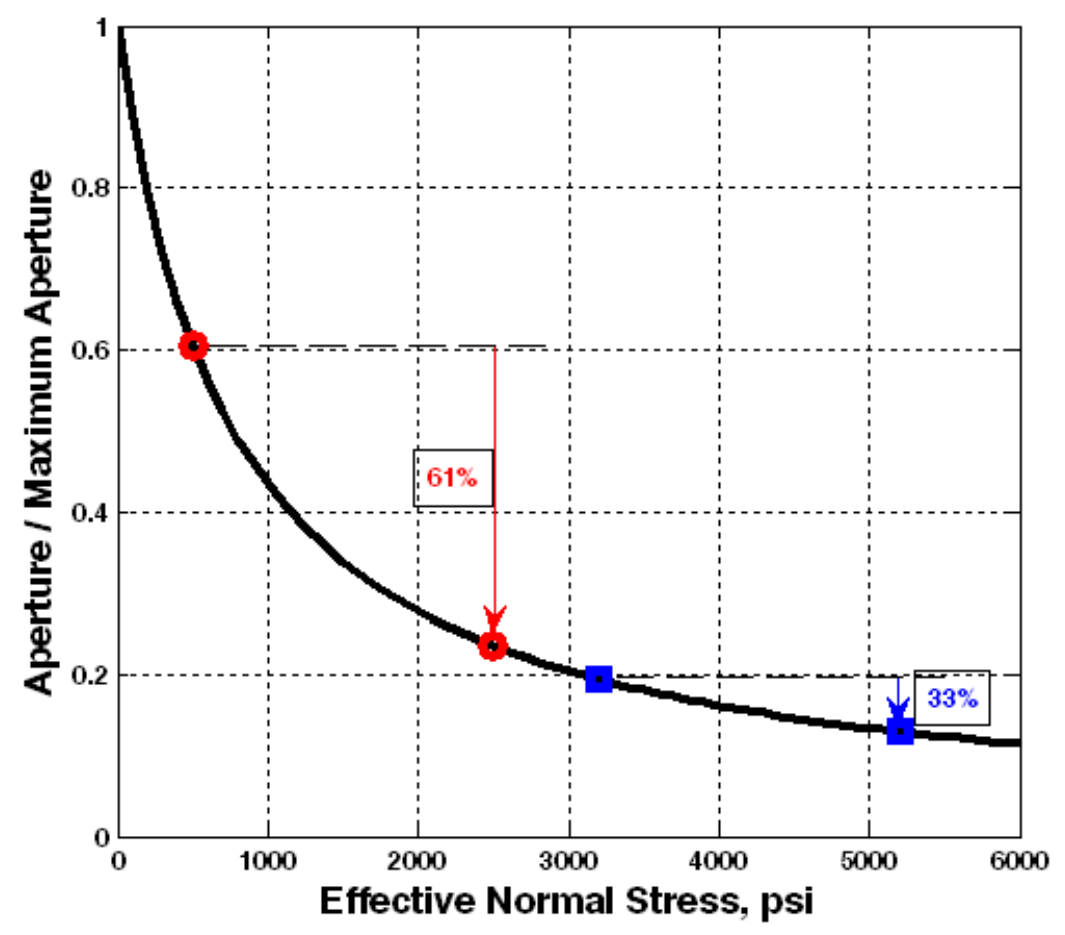

Figure 7.3: Ratio of fracture aperture to the maximum closure calculated by the BartonBandis model. Initial normal stiffness is $7 \times 10^{4} \mathrm{psi} / \mathrm{ft}$ and maximum fracture closure is $0.011 \mathrm{ft}$. 


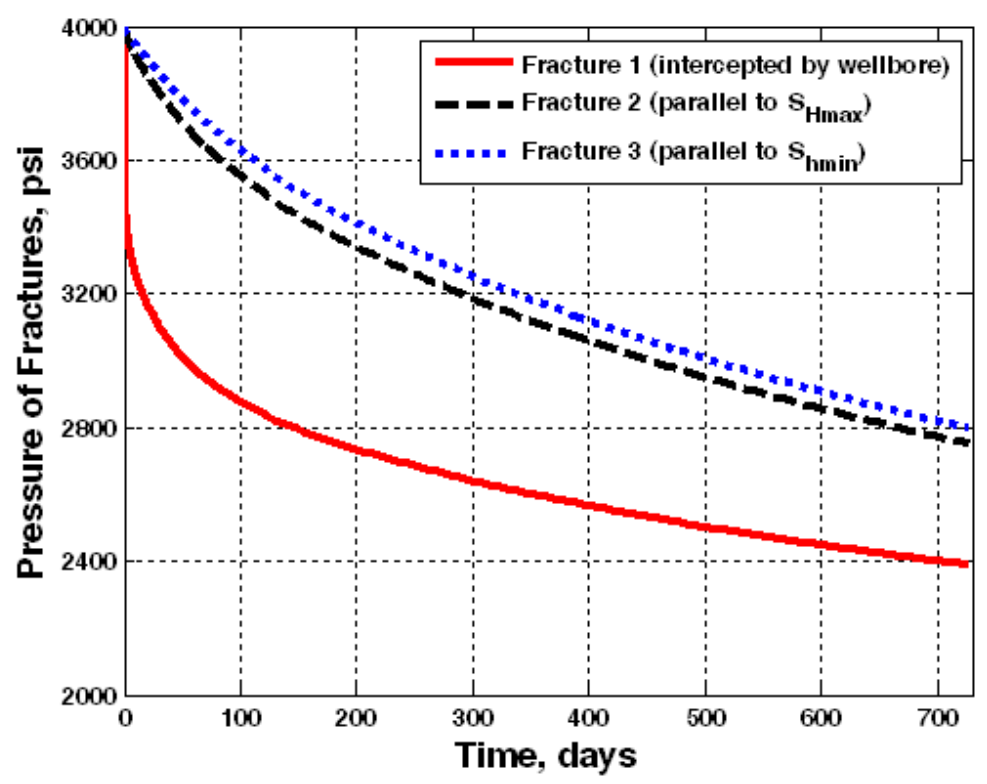

(a)

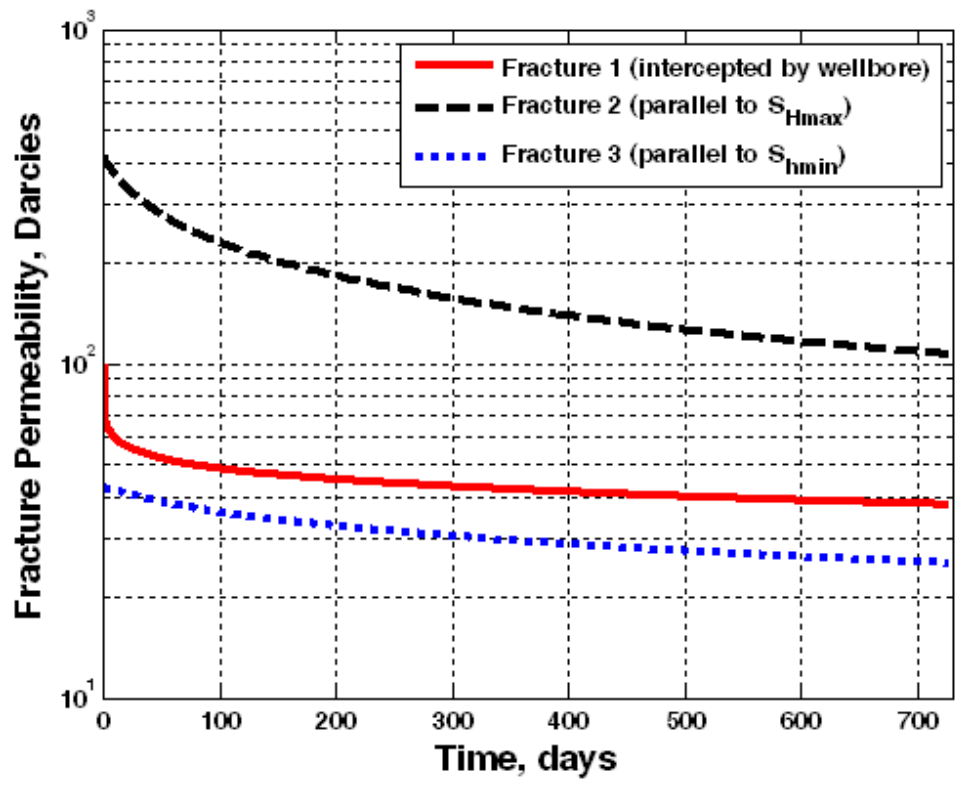

(b)

Figure 7.4: Comparison of (a) pressure and (b) permeability profiles simulated for three fractures, shown with numbers in Figure 7.2, over two years of production. 
Taking into account the dynamic behavior of fractures, slower reservoir depletion and lower hydrocarbon recovery are expected due to reduction of fracture network conductivity, compared to static treatment of the fractures. Figure 7.5 compares the pressure profiles after 150 days of production for dynamic and static cases, demonstrating a smaller depleted area in the dynamic case. The pressures of fractures are also mapped back to their original locations and depicted in the profiles. Furthermore, Figure 7.6 shows the history of oil production rate and cumulative oil production for both cases. When properties of fractures are pressure-dependent, the total oil production after two years is $27.5 \%$ lower than the one associated with the static case. Thus, considering fracture closure induced by production and pressure depletion can be very important in evaluating production from fractured reservoirs. However, dynamic treatment of fractures does not always result in such a substantial difference. The significance of this effect depends on many parameters involved in the dynamic behavior of fractures as well as parameters determining the degree of contribution of fractures in the production. The computational times for the dynamic and static simulations were 1.8 and 2.0 minutes, respectively. 


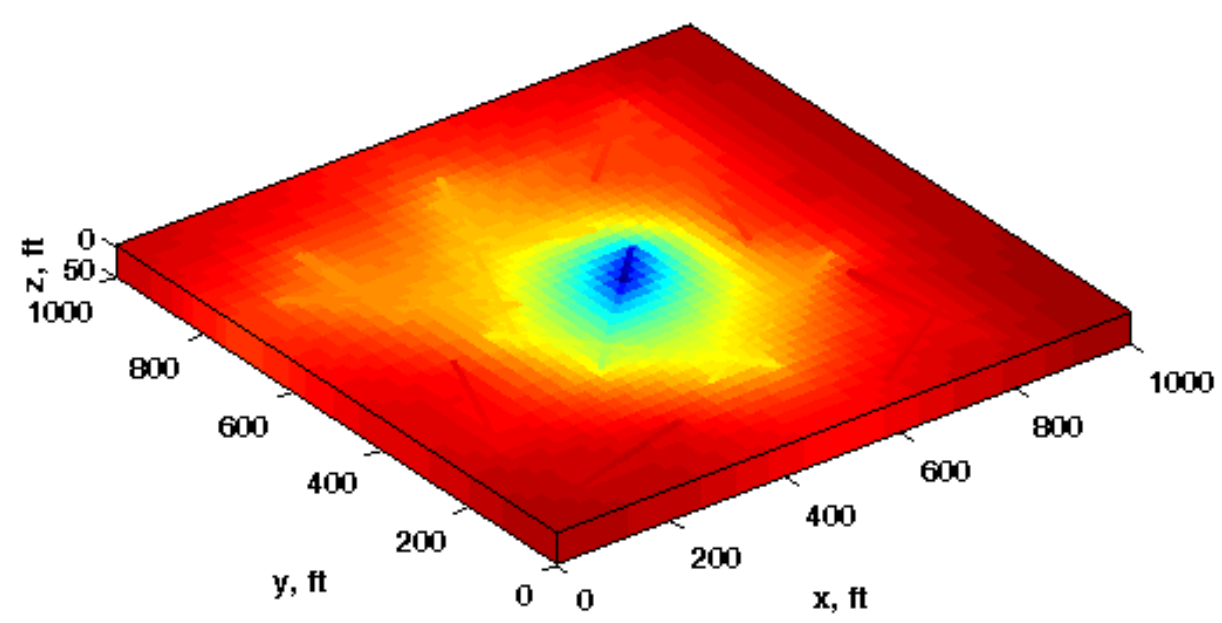

(a)

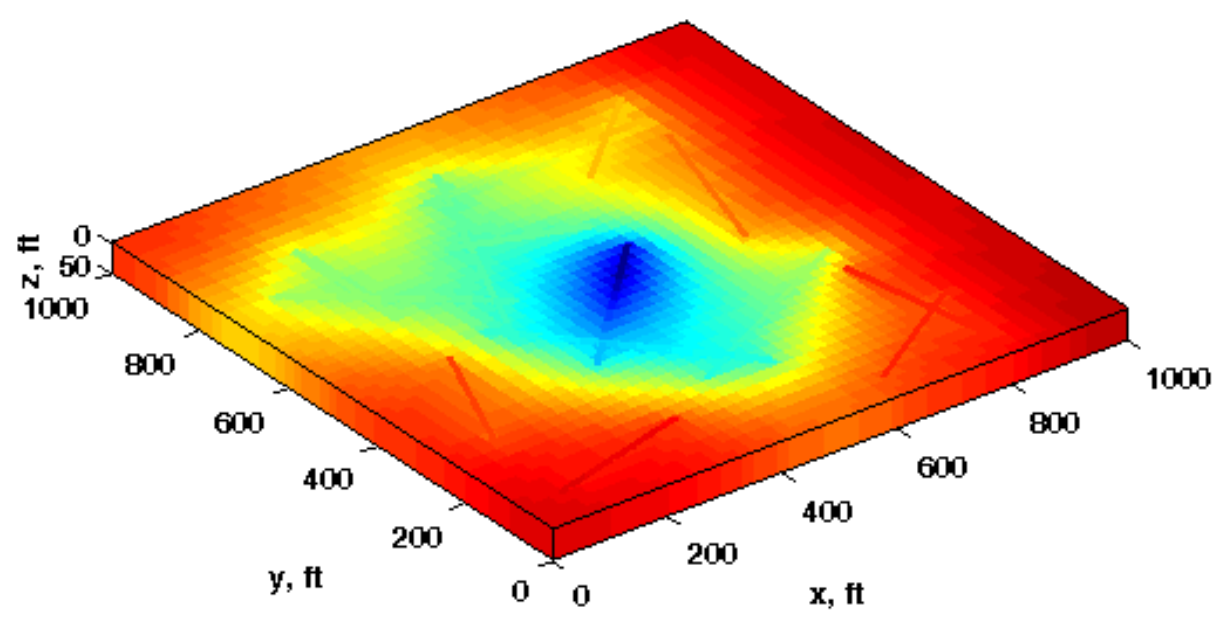

(b)

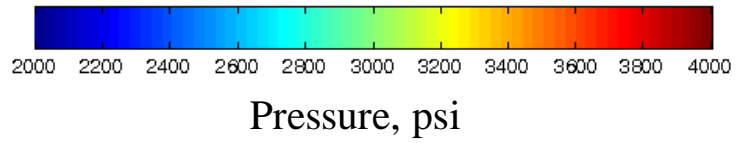

Figure 7.5: Pressure profiles after 150 days of production simulated by the coupled model when (a) fracture properties are pressure-dependent, and (b) fracture properties are constant during production. 


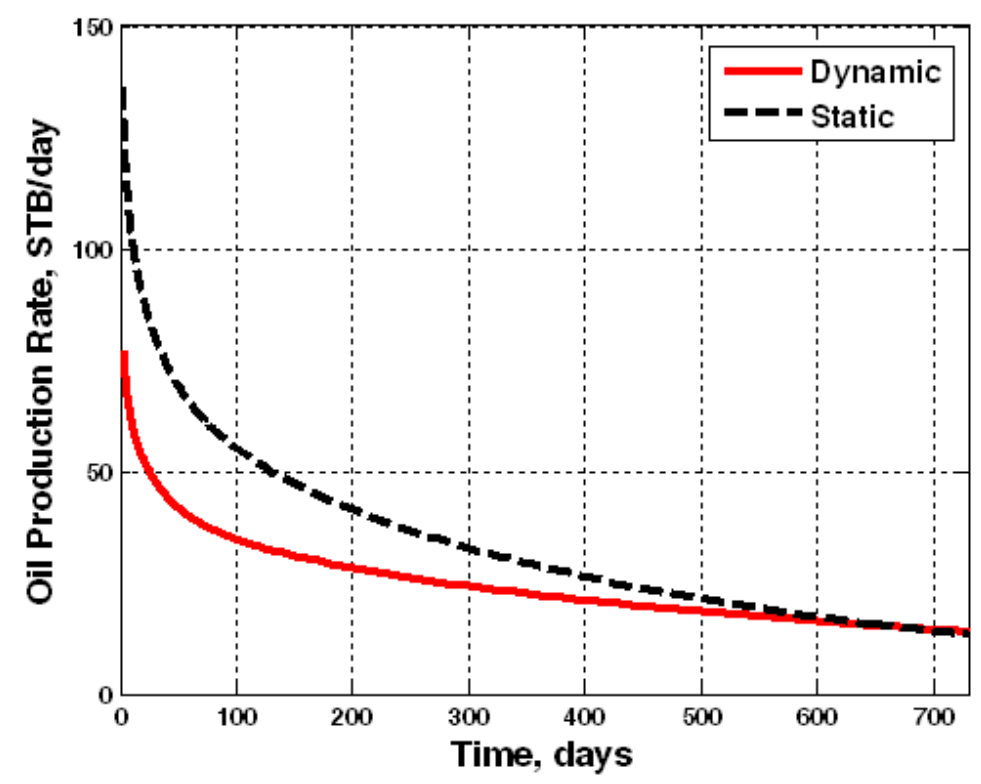

(a)

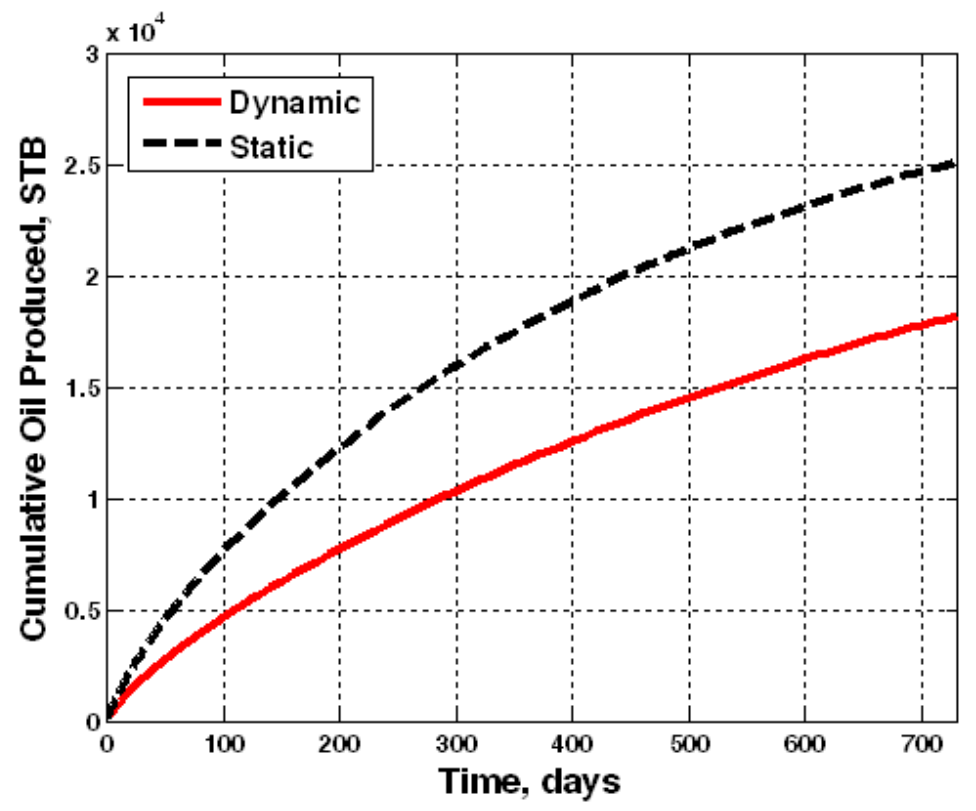

(b)

Figure 7.6: History of (a) oil production rate, and (b) cumulative oil production over two years of production, for the dynamic and static treatments of fractures in the simulations. 
We repeated the simulations for another scenario, which retains the same fracture network and parameters as the base case, but the initial normal stiffness is increased to $3 \times 10^{5} \mathrm{psi} / \mathrm{ft}$. That is, natural fractures are stiffer compared to the previous case. Similar to Figure 7.3, Figure 7.7 shows the ratio of fracture aperture to the maximum closure, calculated by the Barton-Bandis model, for the new joint parameters. Comparison of these figures leads to the conclusion that the initial aperture and permeability of fractures are larger in this case. More importantly, the aperture decline and the permeability loss caused by pressure depletion are smaller in the new scenario. Upon 2000 psi pressure drawdown in fractures, Fracture 2 (in Figure 7.2) would lose $34 \%$ of its initial aperture and $56 \%$ of its initial permeability. Also, Fracture 3 (in Figure 7.2 ) loses $23 \%$ of its initial aperture and $41 \%$ of its initial permeability. As these fractures are stiffer, the reductions in their conductivities are not as severe as those reported for the base case.

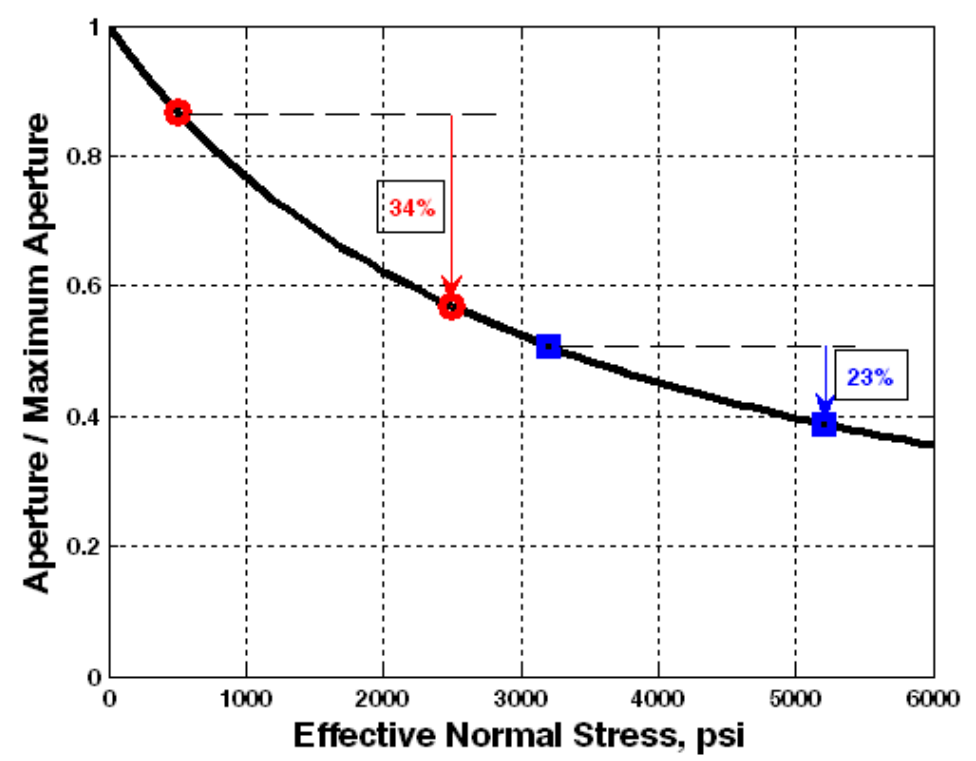

Figure 7.7: Ratio of fracture aperture to the maximum closure calculated by the BartonBandis model. Initial normal stiffness is $3 \times 10^{5} \mathrm{psi} / \mathrm{ft}$ and maximum fracture closure is $0.011 \mathrm{ft}$. 
Figure 7.8 compares the cumulative oil production for dynamic and static treatments of fractures, indicating a much smaller difference between both treatments compared to the base case. When fracture properties are pressure-dependent, the total oil production after 1 and 2 years are $6.6 \%$ and $2.5 \%$ lower than the ones calculated assuming that fracture properties are constant. Consequently, the influence of fracture deformation on production strongly depends on parameters controlling the deformation behavior of fractures, such as the initial normal stiffness.

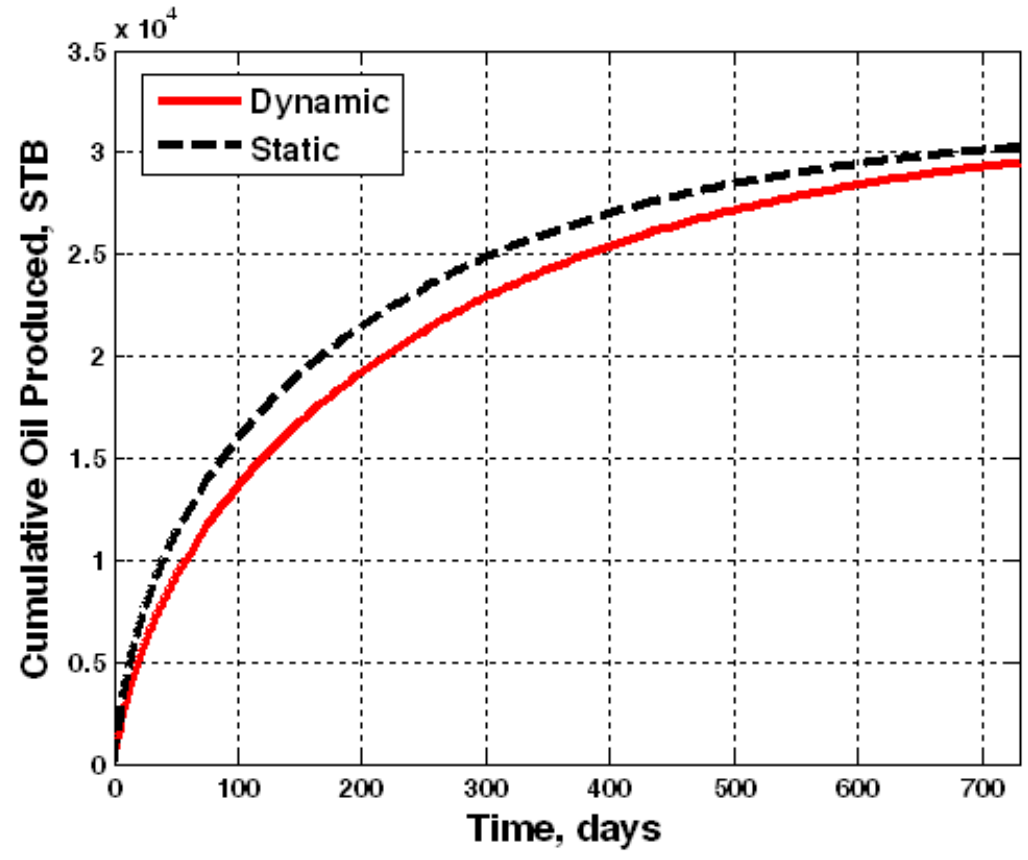

Figure 7.8: History of cumulative oil production over two years of production for the dynamic and static treatments of fractures in the simulations. 
In order to show the applicability and performance of the coupled geomechanicsEDFM approach for realistic simulation of fractured reservoirs, additional simulations were performed on a synthetic 3D model reservoir shown in Figure 7.9. The model reservoir represents a naturally fractured reservoir wherein a large number of fractures with various heights, lengths, orientations, spacings, and network connectivity are present. The reservoir dimensions are $1000 \times 1000 \times 75 \mathrm{ft}$ and a vertical well is located at the center of the reservoir and perforated along the total height of the formation. The model reservoir contains a total of 148 vertical and slanted natural fractures. The matrix grid is $45 \times 45 \times 3$ cells in $\mathrm{x}, \mathrm{y}$, and $\mathrm{z}$ directions, respectively. Some fractures penetrate all three numerical layers while the rest of them penetrate only one or two layers. All parameters in the simulations are identical to those used in the base case.

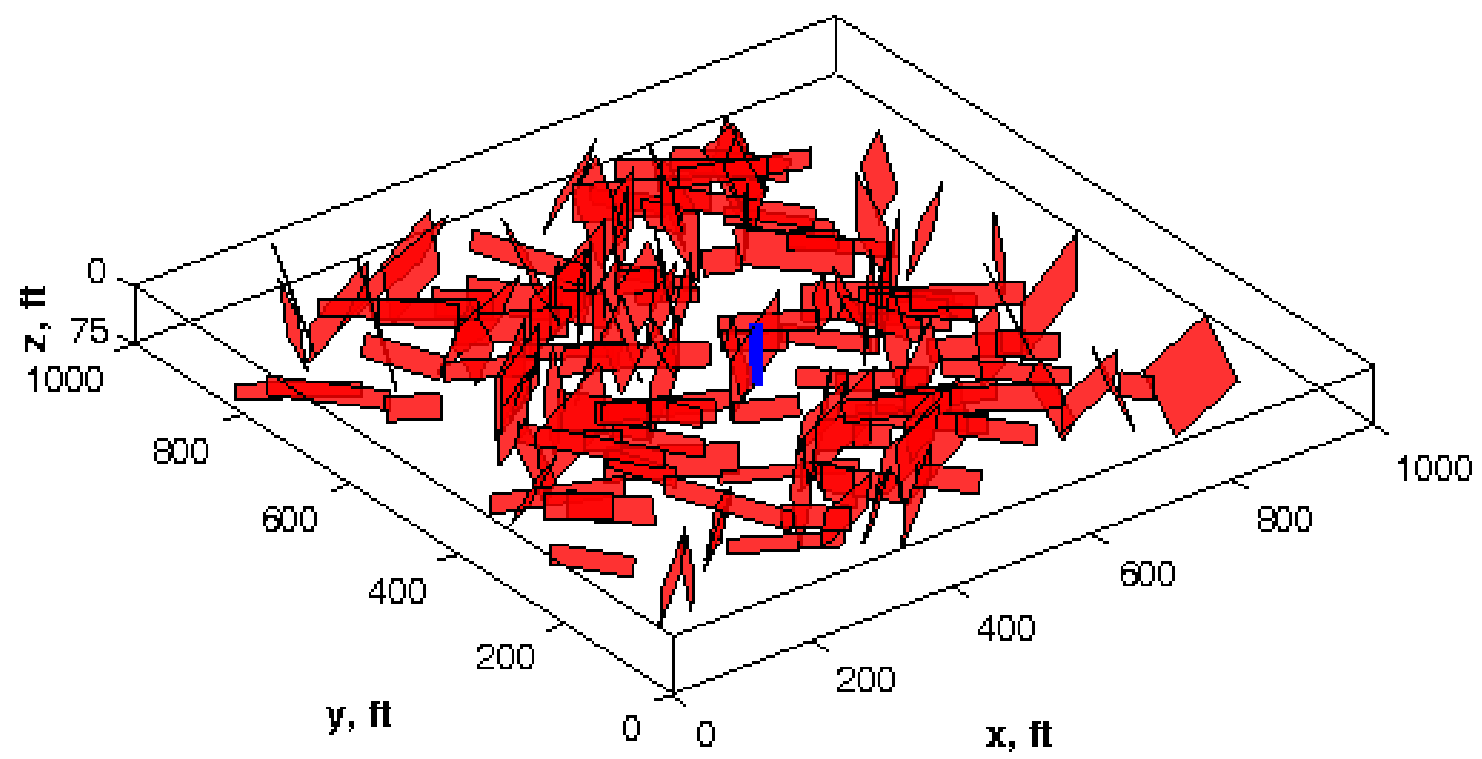

Figure 7.9: A synthetic 3D model reservoir $(1000 \times 1000 \times 75 \mathrm{ft})$ that contains a total of 148 vertical and slanted natural fractures. 
Figure 7.10 shows the pressure profiles after 150 days of production, computed by the coupled geoemchanics-EDFM approach. The first and second rows correspond to the dynamic and static treatments of fractures, respectively. For the sake of completeness, pressure profiles in all three numerical layers are presented in Figure 7.10, depicting different pressure depletion patterns in various layers due to the presence of randomlygenerated natural fractures. Again, slower pressure depletion is observed when fracture properties are pressure-dependent. Also, Figure 7.11 compares cumulative oil production and average reservoir pressure over two years of production for dynamic and static cases. The total oil production after two years calculated by the dynamic treatment of fractures is 0.86 of that computed assuming fracture properties are static. Similarly, the average reservoir pressure drop after two years in the dynamic case is $979 \mathrm{psi}$, which is 0.86 of that for the static case (1135 psi). The computational times for the dynamic and static simulations were 63.8 and 60.4 minutes, respectively, indicating that incorporating dynamic behavior of fractures into EDFM does not degrade the computational performance of EDFM. 

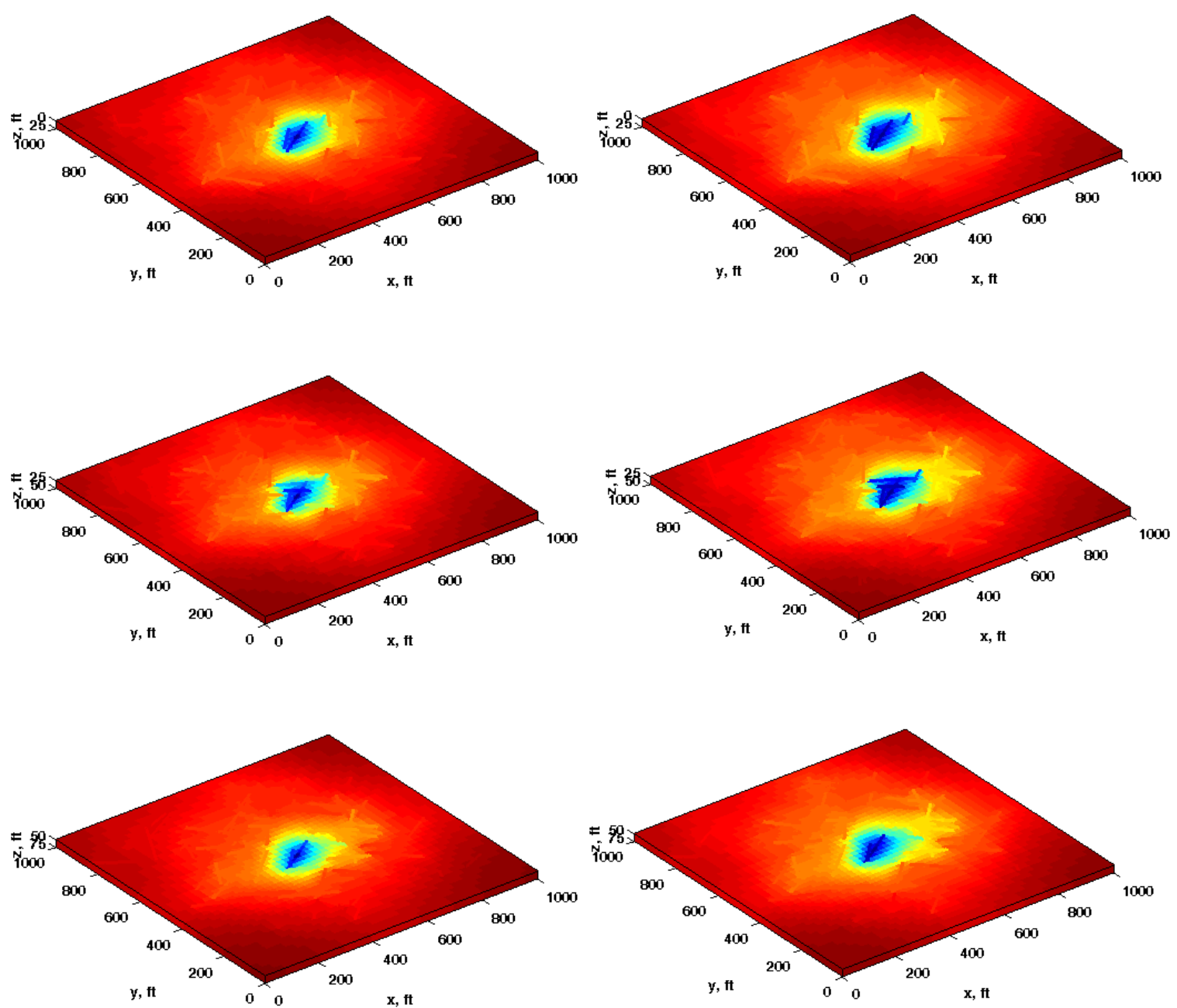

(a)

(b)

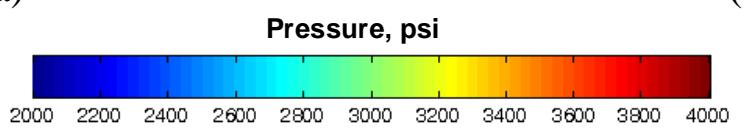

Figure 7.10: Profiles of pressure after 150 days of production for the (a) dynamic and (b) static treatments of fractures. The first, second, and third rows correspond to the top, middle, and bottom layers of the reservoir shown in Figure 7.9. 


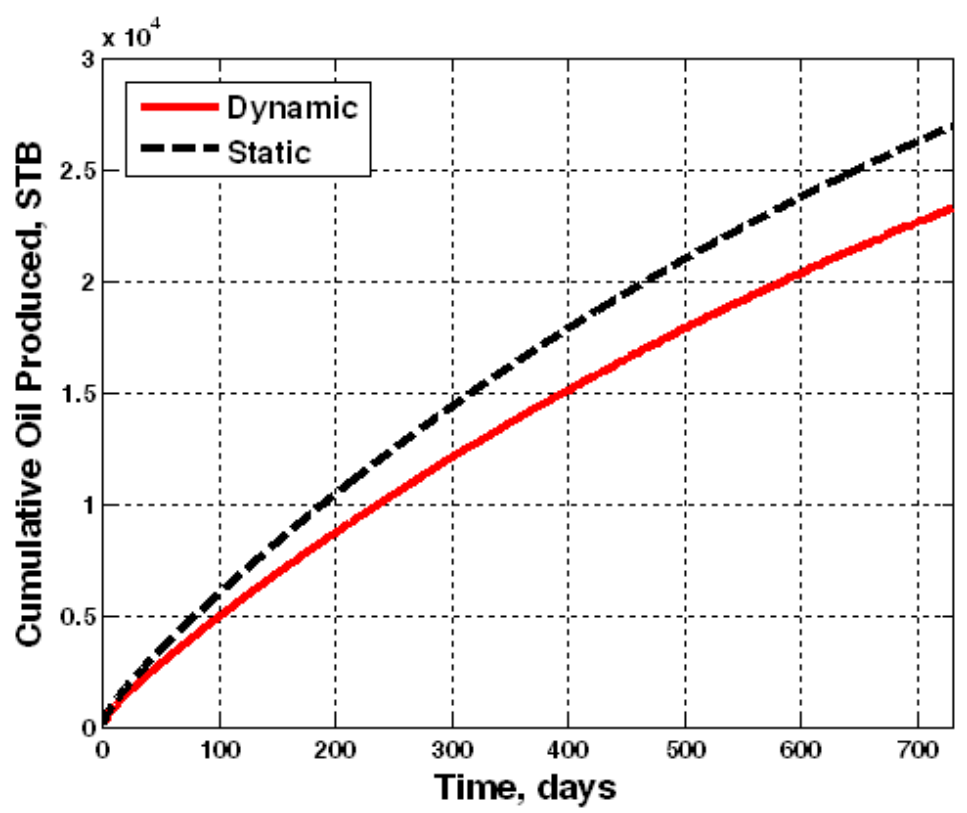

(a)

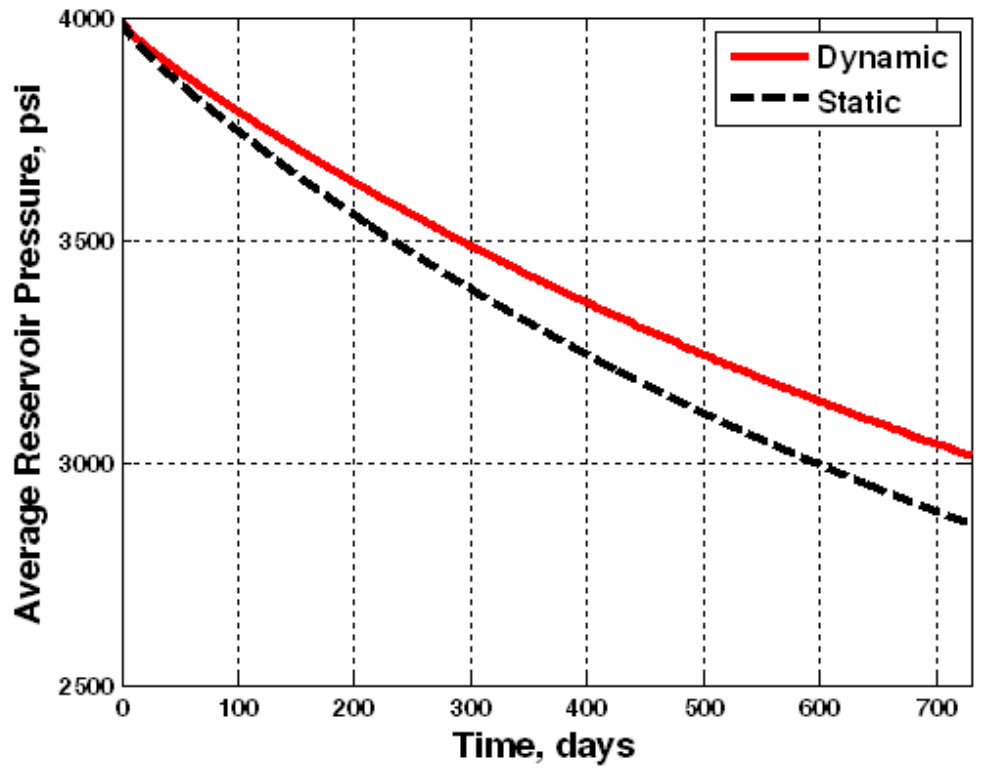

(b)

Figure 7.11: History of (a) cumulative oil production, and (b) average reservoir pressure over two years of production from the model reservoir shown in Figure 7.9. Comparisons are made between the dynamic and static treatments of fractures. 


\subsubsection{Depletion of an Unconventional Gas Reservoir}

The purpose of the second example is to investigate the effect of hydraulic fracture closure resulting from pressure depletion in an unconventional gas reservoir shown in Figure 7.12. The reservoir dimensions are $3000 \times 1500 \times 50 \mathrm{ft}$. The model contains five transverse hydraulic fractures with $600-\mathrm{ft}$ spacing. All vertical fractures intercept a common horizontal well and penetrate the entire height of the formation. Also, they are perpendicular to the minimum horizontal stress, which is 5000 psi. The half-length of all fractures is $150 \mathrm{ft}$. The formation porosity, temperature, and the rock compressibility are $0.03,120^{\circ} \mathrm{F}$, and $1 \times 10^{-6} \mathrm{psi}^{-1}$, respectively. The water saturation in the rock matrix is at the irreducible water saturation of 0.2 and the endpoint relative permeability of gas is 1.0 . The initial reservoir pressure is 3800 psi and the horizontal well produces at a constant bottomhole pressure of 1000 psi. All fractures are propped and thus, we do not use the Barton-Bandis model for the dynamic behavior of hydraulic fractures. Instead, we use Table 7.1, which relates the conductivity of hydraulic fractures (fracture aperture times fracture permeability) to the closure stress acting on them.

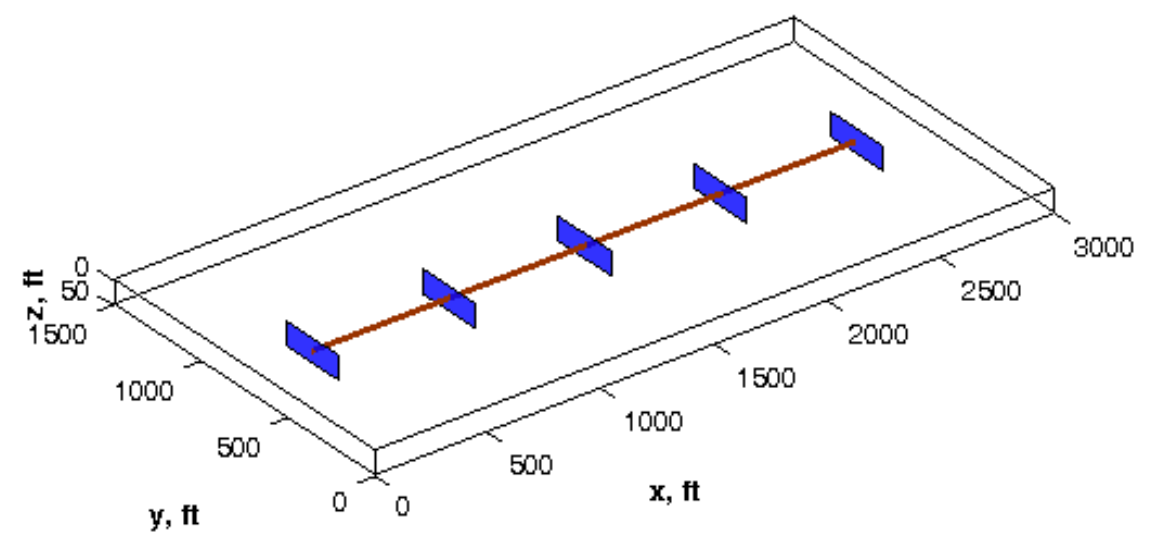

Figure 7.12: A 2D model representing an unconventional gas reservoir, which is considered in the second example. The model comprises a $2400-\mathrm{ft}$ horizontal well (brown line) and five transverse hydraulic fractures (blue planes). 
We examine gas production for two matrix permeabilities, 0.0001 and $0.001 \mathrm{md}$. The matrix grid is $85 \times 31 \times 1$ cells in $\mathrm{x}, \mathrm{y}$, and $\mathrm{z}$ directions, respectively. In order to achieve higher accuracy, the gridblocks around the hydraulic fractures and the wellbore are moderately refined. Figure 7.13 shows the pressure profiles after 15 years of production, simulated by the coupled geomechanics-EDFM approach, for both matrix permeabilities examined. Significantly greater pressure depletion is seen for the case of $0.001 \mathrm{md}$ matrix permeability compared to the case of $0.0001 \mathrm{md}$.

Also, Figure 7.14 compares the cumulative gas production calculated by the dynamic and static treatments of hydraulic fractures for both scenarios. When matrix permeability is $0.0001 \mathrm{md}$, the effect of hydraulic fracture closure with pressure depletion is very small and results in $2.1 \%$ lower gas recovery after 30 years. However, the effect is more pronounced for the case of 0.001 md matrix permeability. That is, total gas production would be overestimated by $5.5 \%$ if we neglect the dependency of hydraulic fracture conductivity on pressure changes. At the beginning, hydraulic fractures in both cases behave like infinite-conductivity fractures. After conductivity reduction due to pressure depletion, hydraulic fractures in the first scenario $\left(k_{m}=0.0001 \mathrm{md}\right)$ still behave close to that of infinite-conductivity fractures, while in the second scenario $\left(k_{m}=0.001\right.$ md), they behave like finite-conductivity fractures, resulting in a considerable difference in production between dynamic and static treatments of hydraulic fractures. Thus, creating sufficiently high-conductivity fractures during stimulation treatment of low permeability reservoirs could mitigate the adverse effect of fracture closure on production to a good extent. The computational times for all four cases presented here were less than 2 minutes. However, more realistic simulation of unconventional gas reservoirs requires incorporating the effect of gas desorption and non-Darcy flow, which were neglected in this example. 


\begin{tabular}{|c|c|c|c|c|c|c|c|}
\hline Closure stress, psi & 1200 & 1500 & 2000 & 2500 & 3000 & 3500 & 4000 \\
\hline Fracture conductivity, md-ft & 21.50 & 12.82 & 6.24 & 2.51 & 1.45 & 0.92 & 0.61 \\
\hline
\end{tabular}

Table 7.1: Conductivity of hydraulic fractures in Figure 7.12 versus closure stress

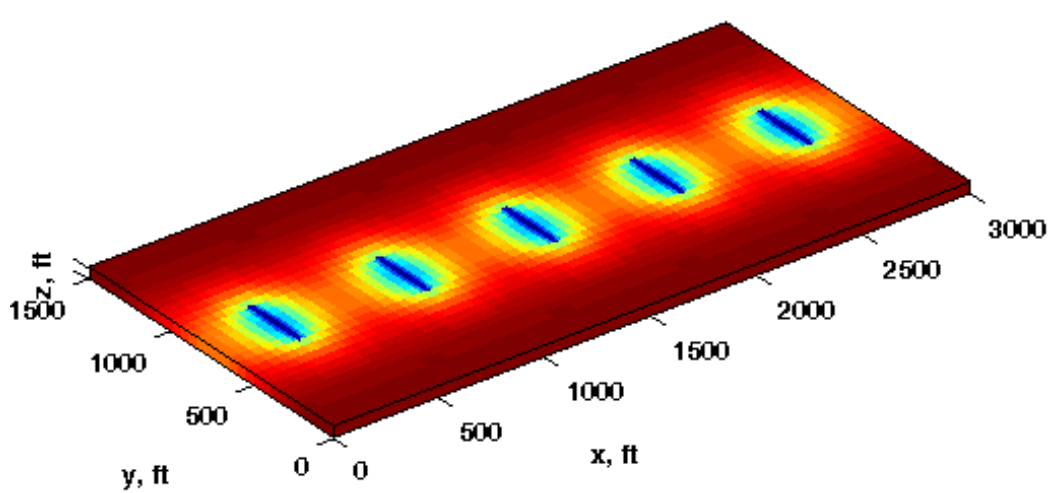

(a)

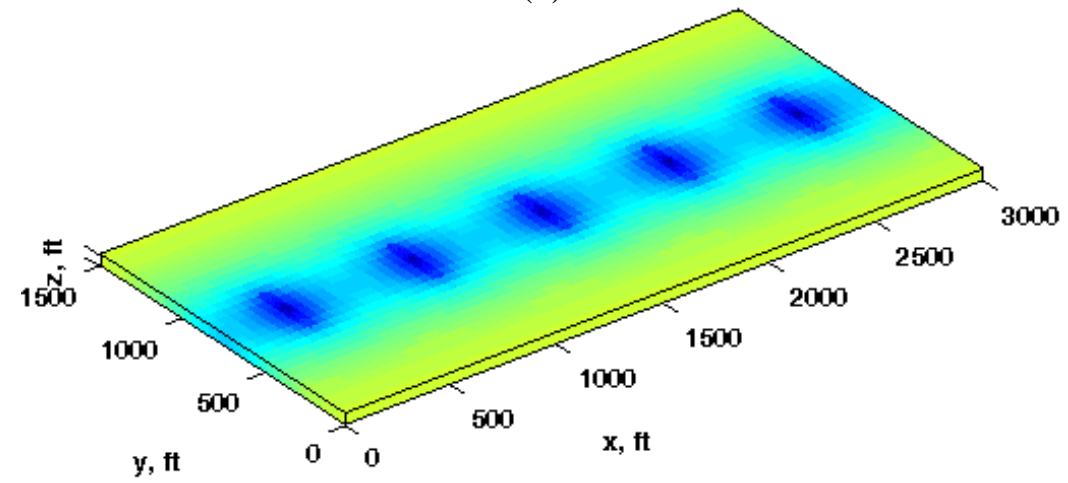

(b)

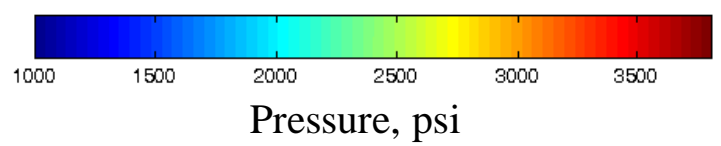

Figure 7.13: Pressure profiles after 15 years of production when fracture properties are pressure-dependent and formation permeability is (a) $0.0001 \mathrm{md}$, and (b) $0.001 \mathrm{md}$. 


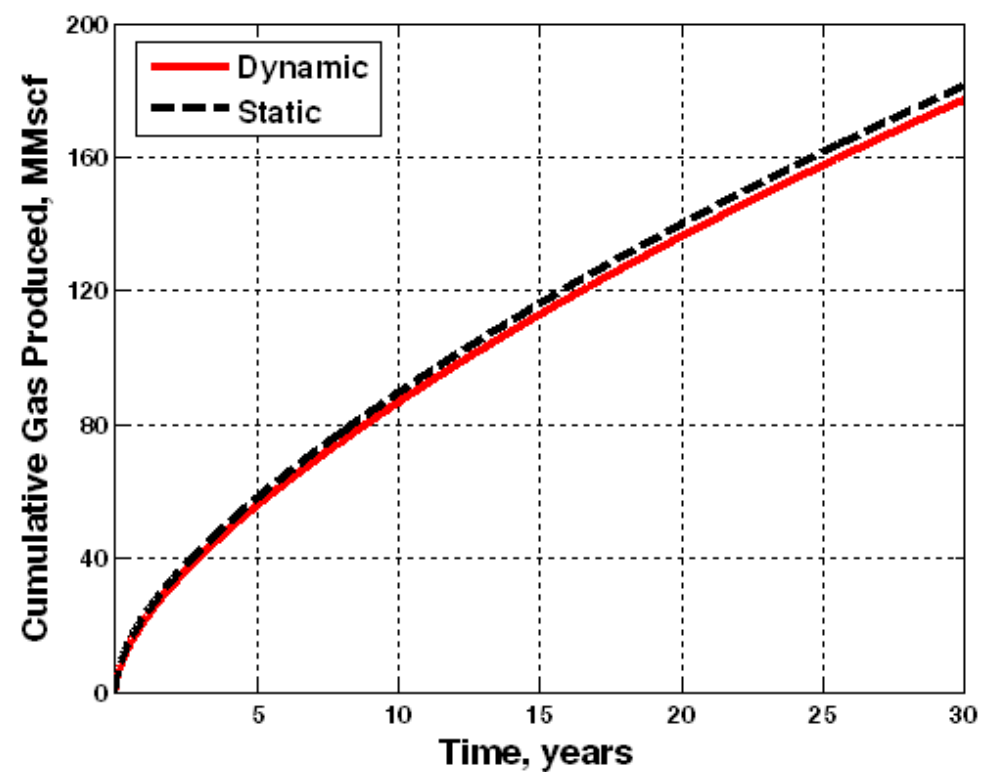

(a)

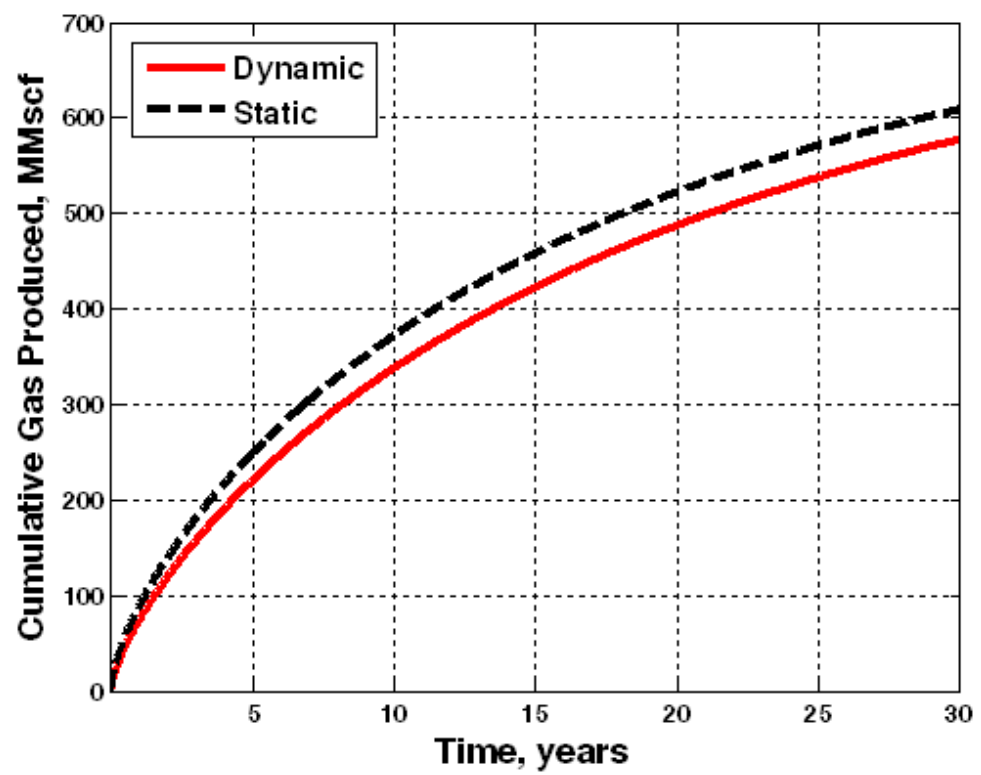

(b)

Figure 7.14: Comparison of the cumulative oil production over 30 years for the dynamic and static treatments of hydraulic fractures when formation permeability is (a) $0.0001 \mathrm{md}$, and (b) $0.001 \mathrm{md}$. 


\subsection{Discussion}

The study presented in this chapter is among the first attempts to couple geomechanics and flow for a discrete fracture model. That is, we incorporated the effect of fracture geomechanics into EDFM in order to simulate naturally fractured reservoirs more accurately. We used the empirical fracture-closure models to account for normal deformation of fractures, which is caused by changes in effective normal stress acting on the fracture and leads to fracture permeability reduction with pressure depletion. Nevertheless, in some special cases where fractures are very weak, fracture permeability could be enhanced by production due to shear deformation. Likewise, dynamic treatment of rock-matrix properties may affect production in fractured systems. Therefore, the developed model can be improved by including matrix geomechanics and fracture shear deformation, which were neglected in this study. However, the effect of shear stress on flow characteristics of fractures is not well understood. Also, we considered the static impact of stress regime on fracture conductivity while, in order to accomplish more realistic simulations of fractured reservoirs, the impact of fractures and production on local changes of stress field should be taken into account. Fully-coupled geomechanicsflow simulations, wherein mechanical deformation of rock and fractures is numerically simulated, can handle the effect of geomechanics more precisely, but they are very complex and computationally-expensive. The coupling approach presented in this study does not degrade the computational performance of a discrete fracture model, even though its measure of accuracy should be evaluated by comparison to a fully-coupled geomechanics-flow model. 


\subsection{Highlights OF THE CHAPTER}

- One advantage of the EDFM approach is its extensibility for various applications by incorporating different physics in the model.

- The dynamic behavior of fractures is incorporated into EDFM. The coupled approach allows considering the impact of stress regime on fluid flow in a $3 \mathrm{D}$ discrete fracture network.

- The non-linear Barton-Bandis joint model is used to represent normal deformation of pre-existing natural fractures. Also, fracture-conductivity tables are allowed to model dynamic behavior of propped hydraulic fractures in stimulated reservoirs.

- Several examples are presented to demonstrate the applicability and performance of the developed model for simulating fractured reservoirs.

- Using the Barton-Bandis model, fractures under lower initial effective normal stress are prone to larger aperture decline and permeability loss with pressure depletion.

- Consideration of dynamic behavior of fractures substantially affects pressure depletion and hydrocarbon recovery. The significance of such effects on production strongly depends on parameters controlling the deformation behavior of fractures.

- Creating sufficiently high-conductivity fractures during stimulation treatment of low permeability reservoirs can mitigate the adverse effect of hydraulic fracture closure to a good extent.

- Implementation of fracture geomechanical effects in EDFM did not degrade the computational performance of EDFM. 


\section{Chapter 8: A Coupled Dual Continuum and Discrete Fracture Model for Application in Unconventional Reservoirs}

Accurate modeling of hydrocarbon production is a necessary, yet challenging step for economic exploitation of unconventional resources. One of the main challenges is to model flow to a horizontal well from a complex network of hydraulic and natural fractures. Many unconventional reservoirs comprise well-developed natural fracture networks with multiple orientations and complex hydraulic fracture patterns based on microseismic data. Conventional dual porosity and dual permeability models are not adequate for modeling these complex networks of natural and hydraulic fractures. Also, it is neither practical nor advantageous to model a large number of pre-existing fractures with a discrete fracture model.

An appropriate approach to model production from these reservoirs is to perform discrete fracture modeling for hydraulic fractures and employ a dual continuum approach for numerous natural fractures. We have developed a coupled dual continuum and discrete fracture model to efficiently overcome challenges for the simulation of unconventional reservoirs. In this chapter, we present the methodology used to implement the coupled approach followed by several example simulations.

\subsection{INTRODUCTION}

The exploitation of unconventional reservoirs is increasingly a major source of short- and long-term energy in the United States. The economic development of unconventional oil and gas hinges in part on effective stimulation of low-permeability 
rock through multi-stage hydraulic fracturing of horizontal wells. To achieve this goal, accurate characterization and simulation of production is necessary for selecting the best stimulation strategy. However, the presence of highly-conductive hydraulic fractures neighboring tight or ultra-tight rock matrix makes numerical simulation of fluid flow very challenging.

Challenges for the simulation of unconventional reservoirs are not limited to the large contrast between the permeability of hydraulic fractures and their neighboring tight rock matrix. Many shale gas units and tight sandstones contain numerous embedded natural fractures, making the numerical simulation of these unconventional reservoirs more challenging. Although recent advances in seismic technology have improved mapping of fracture orientations and densities in unconventional reservoirs, detailed characterization of pre-existing natural fractures is often unavailable. Another substantial challenge for unconventional reservoir simulations is the presence of complex hydraulic fracture geometry. Warpinski et al. (1993) and Fast et al. (1994) presented observations of multi-stranded fracture propagation in cores drilled across expected hydraulic fracture planes and challenged the conventional assumption of single, planar hydraulic fracture propagation. Moreover, complexity of hydraulic fracture propagation became more evident over the last decade with the prevalent application of fracture diagnostic measurements. Maxwell et al. (2002) and Fisher et al. (2005) interpreted microseismic data in the Barnett Shale and observed significant branching of hydraulic fractures, likely the result of opening pre-existing natural fractures. Also, Weng et al. (2011) showed that low in-situ stress anisotropy and pre-existing natural fractures play important roles in creating fracture network complexity. 
Analytical approaches for predicting production in unconventional reservoirs do not consider a complex network of fractures with multiple orientations. Also, Cipolla et al. (2011) showed that dual porosity models are not adequate for simulating complex fracture patterns. They presented flow simulations indicating that neither early-time rate nor estimated ultimate recovery is captured by the dual porosity approach. Hence, we have developed a coupled dual continuum and discrete fracture method to simulate production from unconventional reservoirs. Large-scale hydraulic fractures (macrofractures) are modeled explicitly using EDFM and numerous small-scale natural fractures (micro-fractures) are modeled using a dual continuum approach.

In this chapter, we first describe the methodology used for dual continuum modeling of numerous small-scale natural fractures. Then, we consider a synthetic tight oil reservoir including multi-stage hydraulic fractures and numerous natural fractures to evaluate the accuracy and performance of the coupled approach by comparing its results with the results using the EDFM approach. We also present several examples to demonstrate the applicability and robustness of the hybrid approach for simulating unconventional reservoirs. We examine multi-stage hydraulic fractures with multiple configurations in the presence of numerous pre-existing fractures. We also examine production from a tight gas reservoir wherein hydraulic fractures partially penetrate the formation height. Finally, we discuss the physics, which may affect fluid flow in unconventional reservoirs, leading to the conclusion that the hybrid model presented in this chapter can be easily extended to develop a complete and robust unconventional reservoir simulator. 


\subsection{Methodology}

Large-scale hydraulic fractures provide the main conduits for production of unconventional resources. Thus, a discrete fracture model that can accurately evaluate production from these fractures is preferred to a dual continuum model. Moreover, DFMs allow for complex hydraulic fracture geometry that exists in shales. Compared to hydraulic fractures, numerous small-scale natural fractures play a secondary, yet important role in unconventional oil and gas recovery. Detailed characterization of preexisting natural fractures is still challenging for geologists and engineers so that it is neither practical nor advantageous to model a large number of natural fractures with a discrete fracture model. Hence, a dual continuum approach is ideal for modeling these numerous small fractures. The approach used here is to couple a DFM to account for the large-scale hydraulic fractures with a dual continuum model that accounts for flow in the naturally fractured networks. We use the EDFM approach for the large-scale hydraulic fractures. As demonstrated in the previous chapters, EDFM allows for not only transverse and longitudinal hydraulic fractures but also macro-fractures of any orientation. Therefore, the coupled model is a type of triple porosity approach, including three domains:

1. Matrix domain

2. Discrete-fracture domain

3. Continuum-fracture domain

The same gridblock sizes are used for all three domains; however, there is no constraint for three grid sizes to be the same in the coupled model. Because of the triple porosity nature of the model, we must calculate the appropriate transmissibilities between the various types of fractures and matrix. 
To illustrate the methodology used in the coupled model, Figure 8.1a shows a synthetic 2D model comprising a 2000-ft horizontal well (brown line), seven transverse hydraulic fractures (blue lines) with 300-ft fracture spacing, and 1819 small natural fractures (black lines) with various orientations. Figure $8.1 \mathrm{~b}$ depicts an enlarged region of the model in Figure 8.1a showing the grid boundaries and the embedded natural fractures. This figure indicates that every gridblock may contain multiple natural fractures, which is why a continuum model for those fractures is applicable.

The transport parameters between matrix and discrete-fracture domains are calculated using the same methodology described for the EDFM approach in Chapter 4. In order to account for the effect of small natural fractures in the coupled approach, we need to properly calculate the following transport parameters associated with the third domain (continuum-fracture domain):

- Transmissibility between fracture cells in the continuum-fracture domain and the corresponding matrix gridblocks.

- Transmissibility between adjacent fracture cells in the continuum-fracture domain.

- Transmissibility between a fracture cell in the continuum-fracture domain and a fracture control volume in the discrete-fracture domain if any embedded natural fracture within a gridblock intersects a hydraulic fracture segment. 


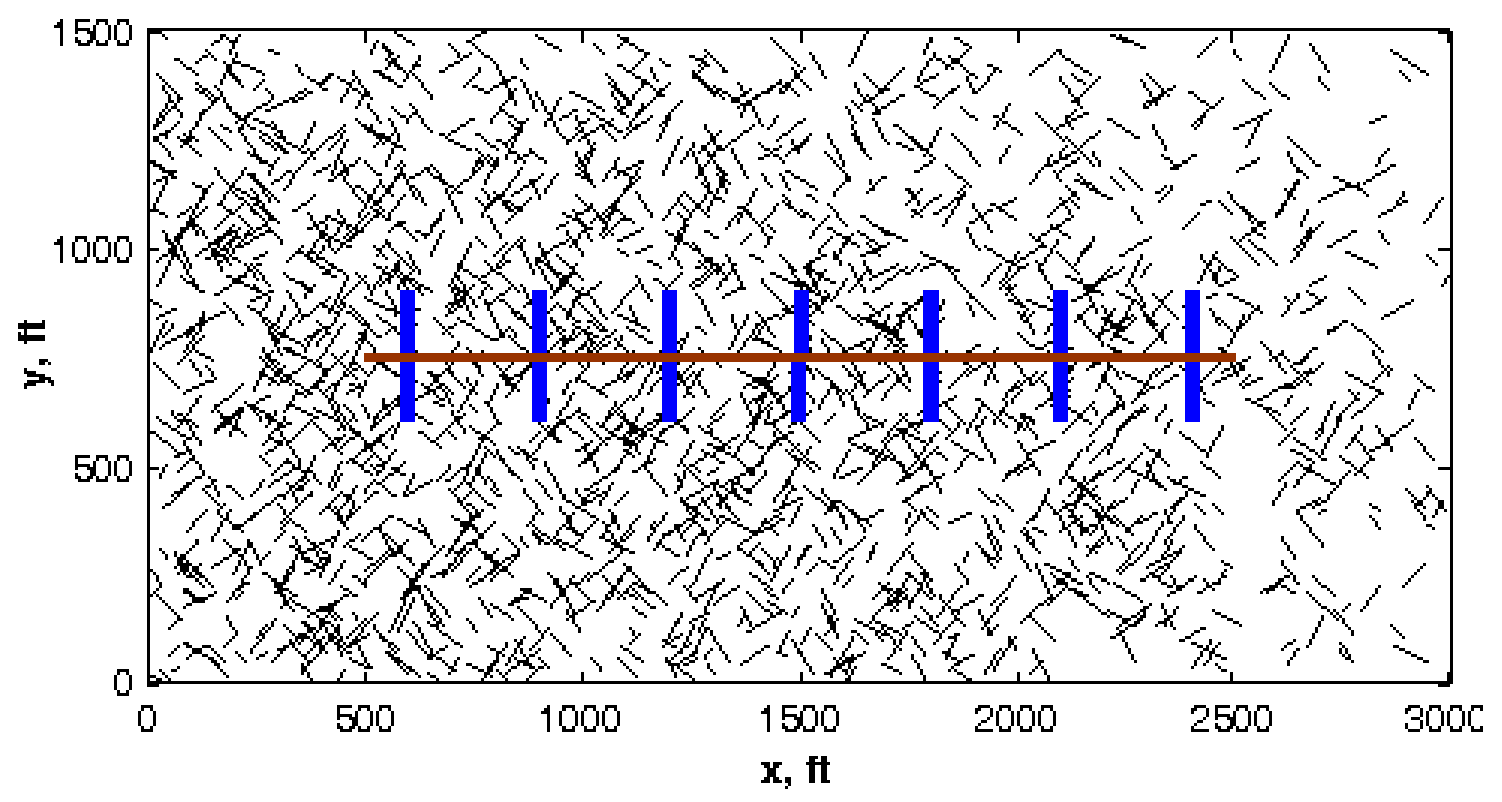

(a)

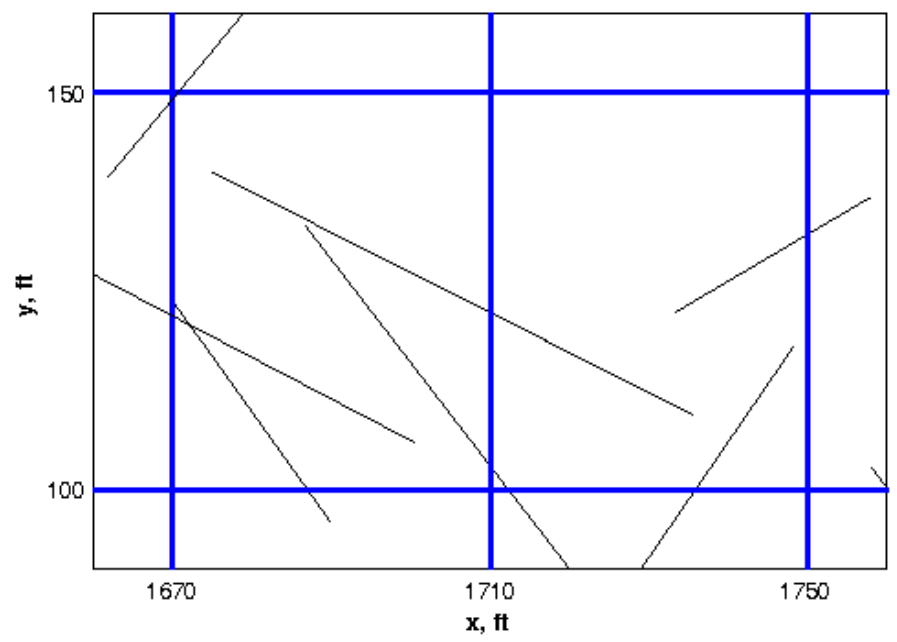

(b)

Figure 8.1: (a) A synthetic 2D reservoir used to illustrate the methodology applied in the coupled model. The model comprises a 2000- $\mathrm{ft}$ horizontal well (brown line), 7 transverse hydraulic fractures (blue lines), and 1819 small-scale natural fractures (black lines). (b) An enlarged region of the model in Figure 8.1a showing the grid boundaries and the embedded natural fractures. 


\subsubsection{Transport Parameters for Dual Continuum Modeling}

In this section, we describe a systematic approach to calculate the aforementioned transport parameters. The subscripts $m, F$, and $f$ in the forthcoming equations refer to the cells in the matrix, discrete-fracture, and continuum-fracture domains, respectively. In Chapter 4, we described the concept of non-neighboring connection (NNC) used in EDFM and the transmissibilities associated with NNC pairs. In the coupled model, we consider a matrix gridblock and its corresponding continuum-fracture gridblock as a NNC pair. Fluid communication between them is accounted for in the NNC term shown in Equation 4.2. The transmissibility between this NNC pair is a weighted sum of transmissibility of individual embedded fractures with the corresponding matrix gridblock and is given by

$$
\begin{aligned}
& T_{m-f}=\sum_{i=1}^{N_{n f}} w_{i} T_{m-f, i}, \\
& T_{m-f, i}=\frac{k_{m-f, i} A_{f, i}}{d_{m-f, i}}
\end{aligned}
$$

where $N_{n f}$ is the number of embedded natural fractures in the gridblock, $w_{i}$ is the volume of the $i$-th fracture bounded in that gridblock divided by the total volume of fractures in that cell, and $T_{m-f, i}$ is the transmissibility of the $i$-th fracture with the matrix gridblock. Subsequently, $k_{m-f, i}$ is the harmonic average of the matrix and fracture permeabilities (which is close to the matrix permeability when the natural fracture permeability is much greater than the matrix permeability), $A_{f, i}$ is the fracture surface area in the gridblock, and $d_{m-f, i}$ is the average normal distance of the fracture from the matrix gridblock calculated using Equation 4.4. Detailed description of parameters used in Equation 8.2 is presented in Chapter 4 (see Section 4.2.3.1). 
Furthermore, if any natural fracture embedded in a gridblock intersects a hydraulic fracture segment in that cell, there should be a connection between the continuum-fracture gridblock and the corresponding fracture control volume in the discrete-fracture domain through a NNC. The transmissibility between this NNC pair is a weighted sum of transmissibility of individual embedded fractures with the hydraulic fracture segment and is given by

$$
T_{F-f}=\sum_{i=1}^{N_{n f}} w_{i} T_{F-f, i}
$$

where $T_{F-f, i}$ is the transmissibility of the $i$-th fracture with the hydraulic fracture segment approximated using Equations 4.5 and 4.6 (see Section 4.2.3.2). Clearly, $T_{F-f, i}$ is zero if two fractures do not intersect within the gridblock.

In order to calculate the transmissibility of two neighboring fracture cells in the continuum-fracture domain, we use the following equation:

$$
\begin{aligned}
& T_{f_{1}-f_{2}}=\sum_{f=1}^{N_{f_{1}-f_{2}}} w_{f} T_{f}, \\
& T_{f}=\frac{k_{f} \omega_{f} L_{f}}{d_{f}},
\end{aligned}
$$

where $N_{f_{1}-f_{2}}$ is the number of natural fractures crossing both neighboring gridblocks. Subsequently, $w_{f}$ is the product of the fracture $w_{i}$-values in two neighboring gridblocks, $k_{f}$ is the fracture permeability, $\omega_{f}$ is the fracture aperture, $L_{f}$ is the length of the intersection line of the fracture plane with the common face of two neighboring gridblocks, and $d_{f}$ is the distance between the centers of two fracture segments (see Section 4.2.4). If none of the embedded natural fractures in a gridblock extends to a neighboring gridblock, then the transmissibility between two gridblocks in the 176 
continuum-fracture domain is zero. For instance, the right gridblock in Figure $8.1 \mathrm{~b}$ has zero transmissibility with the gridblock above it.

The porosity of a fracture cell in the continuum-fracture domain is the total volume of natural fractures embedded in a gridblock divided by the bulk volume of the gridblock. Hence, if a gridblock contains no embedded natural fractures, then the corresponding fracture cell in the continuum-fracture domain is treated as a null block in the fluid-flow simulations to avoid increased computational time. We remark here that the intersections between embedded natural fractures are neglected in the calculation of the transmissibilities described above.

It can be easily inferred from the above-mentioned equations that the parameters for dual continuum modeling of numerous natural fractures are derived by upscaling the EDFM equations. Consequently, we have extended our pre-processing code, previously developed for EDFM (see Section 4.3), to incorporate the required calculations associated with the fracture gridblocks in the continuum-fracture domain. The entire model, including the matrix gridblocks, discrete-fracture control volumes, and continuum-fracture gridblocks, is then an input into a reservoir simulator, which allows for non-neighboring connections and transmissibility modifiers (such as commercial reservoir simulators). The governing equations for the fracture cells in the continuumfracture domain are similar to those described for the matrix and discrete-fracture domains, implying that Darcy's law is used in the continuum-fracture domain. 


\subsection{ACCURACY AND PERFormance OF THE COUPLED APPROACH}

In this section, we evaluate the accuracy and performance of the coupled dual continuum and EDFM approach. Figure 8.2 shows a synthetic 2D reservoir comprising a 660 - $\mathrm{ft}$ horizontal well, four identical transverse hydraulic fractures with 220 - $\mathrm{ft}$ spacing, and 740 small-scale natural fractures. The reservoir dimensions are $1200 \times 800 \times 30 \mathrm{ft}$. The half-length, width, and permeability of each hydraulic fracture are $160 \mathrm{ft}, 0.025 \mathrm{ft}$, and 10 Darcies, respectively. All fractures completely penetrate the height of the formation and there is no additional perforated interval along the well. The reservoir fluid is oil with specific gravity of 0.82 and viscosity of $0.5 \mathrm{cp}$, and the formation porosity and permeability are 0.08 and $0.001 \mathrm{md}$, respectively, representing a tight oil reservoir. Also, the formation temperature and the rock compressibility are $120^{\circ} \mathrm{F}$ and $8 \times 10^{-6} \mathrm{psi}^{-1}$, respectively. The water saturation in rock matrix is at the irreducible water saturation of 0.2 and the endpoint relative permeability of oil is 0.8 . The aperture and permeability of all natural fractures are assumed to be identical and equal to $0.003 \mathrm{ft}$ and 1 Darcy, respectively. The initial reservoir pressure is 4000 psi and the horizontal well produces at a constant bottomhole pressure of 2000 psi, which is still above the bubble point pressure. Table 8.1 summarizes the properties used in the simulations of this section.

In order to provide a reference solution to evaluate the accuracy of the coupled method, we perform an EDFM simulation in which all hydraulic and natural fractures are modeled explicitly. For the EDFM simulation, a $150 \times 101 \times 1$ matrix grid is used. Also, we examine three simulations using the coupled model with different grid resolutions. The first, second, and third simulations use $30 \times 21 \times 1,60 \times 41 \times 1$, and $100 \times 67 \times 1$ matrix grids, respectively. The descriptions of matrix grid for all simulations are given in Table 8.2. Figure 8.3 presents profiles of pressure in the matrix domain after 200 days of production 
calculated by EDFM and coupled simulations. Also, Figure 8.4 compares the oil production rate over six years of production.

As it can be seen, the results of the coupled approach which correspond to the $60 \times 41 \times 1$ and $100 \times 67 \times 1$ matrix grids are in close agreement with the results using the EDFM approach. The computational time for the EDFM simulation was 74.1 minutes and the computational times for three simulations using the coupled approach were 2.1, 6.3, and 32.6 minutes for various refinements as stated above.

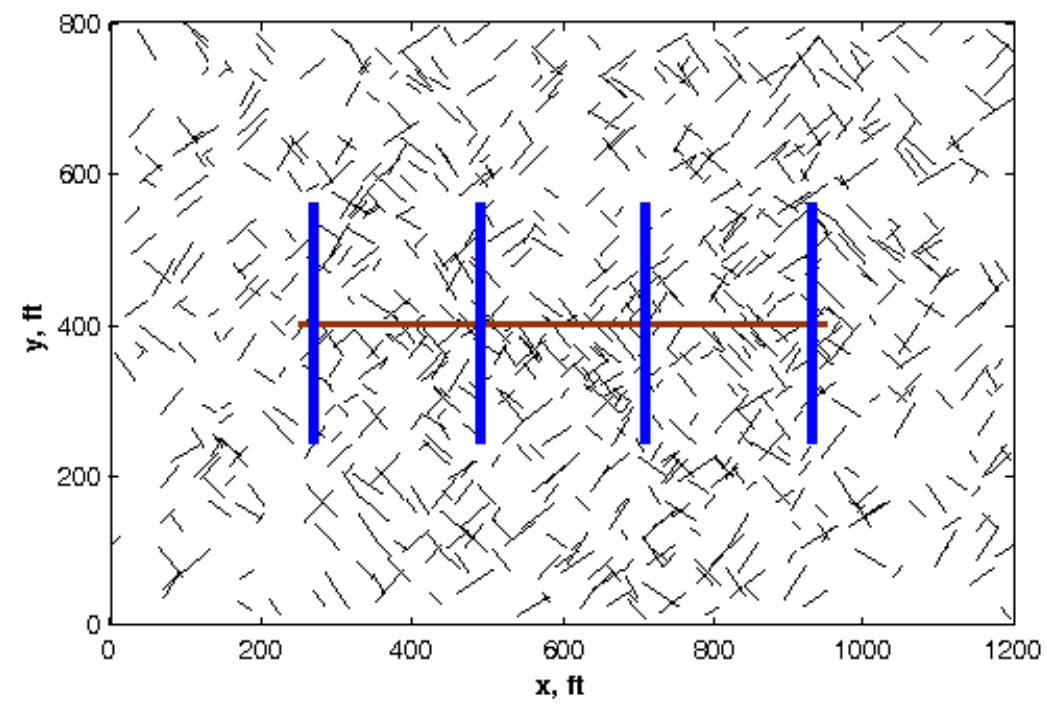

Figure 8.2: A synthetic 2D reservoir used to evaluate the accuracy of the coupled approach. The model comprises a 660-ft horizontal well (brown line), four transverse hydraulic fractures (blue lines), and 740 small-scale natural fractures (black lines). 


\begin{tabular}{|c|c|c|c|}
\hline Variable & Value & Variable & Value \\
\hline Matrix porosity & 0.08 & Specific gravity of oil & 0.82 \\
\hline Matrix permeability & $0.001 \mathrm{md}$ & Oil viscosity & $0.5 \mathrm{cp}$ \\
\hline Hydraulic fracture half-length & $160 \mathrm{ft}$ & Reservoir temperature & $120^{\circ} \mathrm{F}$ \\
\hline Hydraulic fracture width & $0.025 \mathrm{ft}$ & Rock compressibility & $8 \times 10^{-6} \mathrm{psi}^{-1}$ \\
\hline Hydraulic fracture permeability & $10 \mathrm{D}$ & Initial water saturation & 0.2 \\
\hline Natural fracture aperture & $0.003 \mathrm{ft}$ & Initial reservoir Pressure & $4000 \mathrm{psi}$ \\
\hline Natural fracture permeability & $1 \mathrm{D}$ & Producer BHP & $2000 \mathrm{psi}$ \\
\hline
\end{tabular}

Table 8.1: Summary of properties used in the simulations performed for the model reservoir shown in Figure 8.2.

\begin{tabular}{|l|c|l|}
\hline Simulation approach & $\Delta \mathrm{x}(\mathrm{ft})$ & \multicolumn{1}{|c|}{$\Delta \mathrm{y}(\mathrm{ft})$} \\
\hline EDFM $-150 \times 101 \times 1$ & $150 \times 8$ & $49 \times 8,6,4,6,49 \times 8$ \\
\hline Coupled $-100 \times 67 \times 1$ & $100 \times 12$ & $33 \times 12,8,33 \times 12$ \\
\hline Coupled $-60 \times 41 \times 1$ & $60 \times 20$ & $19 \times 20,16,8,16,19 \times 20$ \\
\hline Coupled $-30 \times 21 \times 1$ & $30 \times 40$ & $9 \times 40,30,20,30,9 \times 40$ \\
\hline
\end{tabular}

Table 8.2: Grid description for the simulations performed for the model shown in Figure 8.2. 


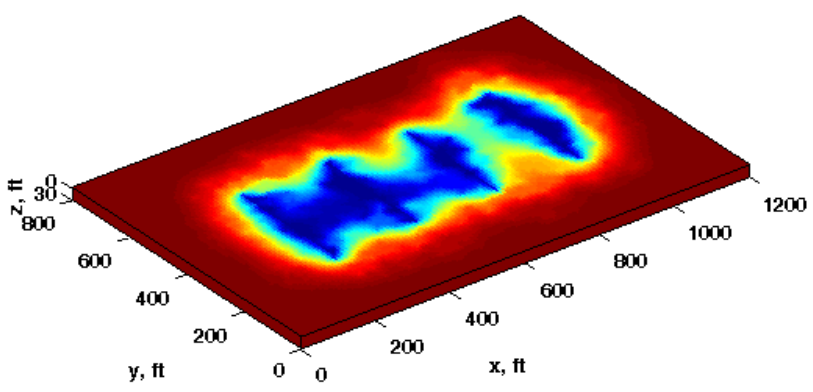

(a)

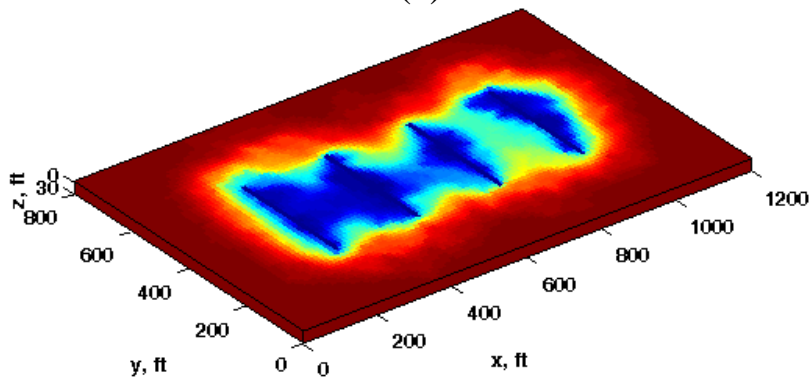

(b)

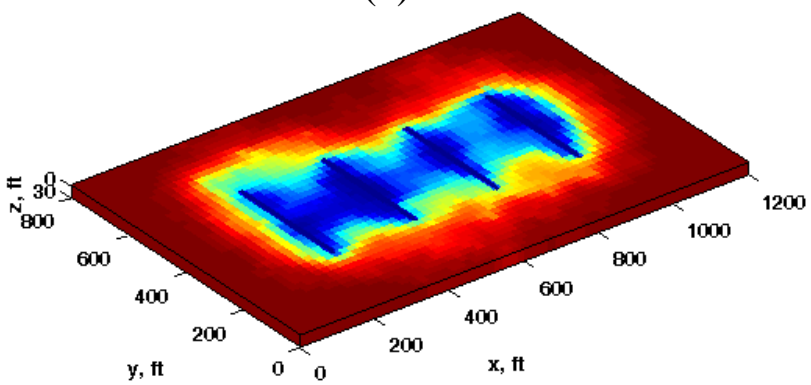

(c)

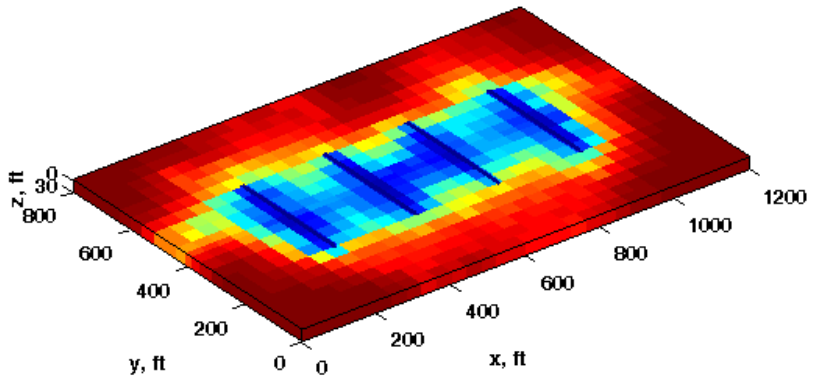

(d)

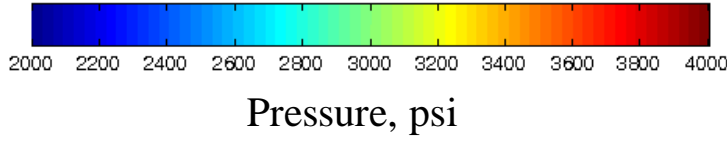

Figure 8.3: Pressure profiles simulated by the (a) EDFM approach, (b) coupled model using a $100 \times 67 \times 1$ matrix grid, (c) coupled model using a $60 \times 41 \times 1$ matrix grid, and (d) coupled model using a $30 \times 21 \times 1$ matrix grid after 200 days of production for the reservoir shown in Figure 8.2. 


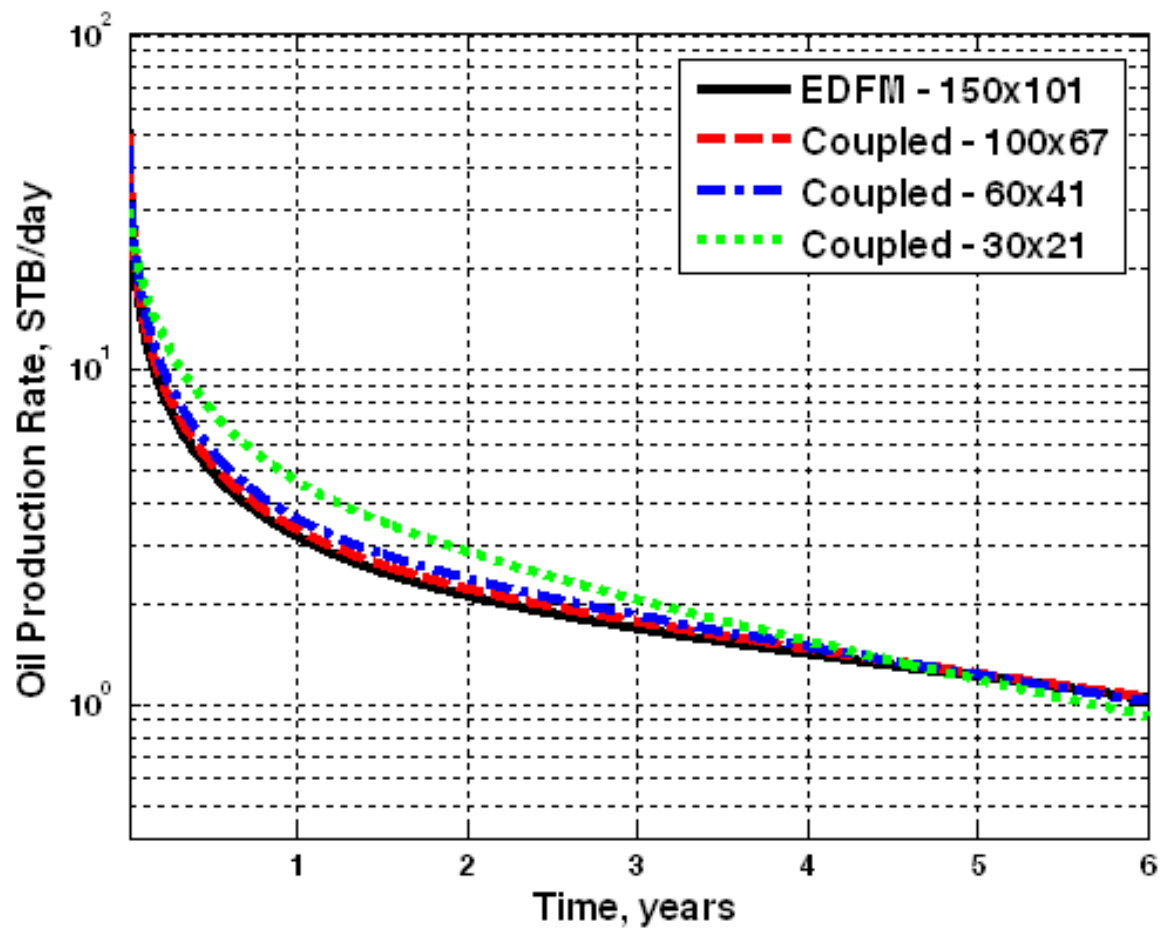

Figure 8.4: Comparison of oil production rate over six years of production for the reservoir shown in Figure 8.2 calculated by the EDFM approach and three simulations using the coupled approach. 


\subsection{EXAMPLE Simulations}

In this section, we present several examples to demonstrate the applicability, robustness, and performance of the coupled model for simulating production from unconventional reservoirs.

\subsubsection{D Tight Oil Reservoir}

In the first example, we simulate production from the synthetic $2 \mathrm{D}$ reservoir shown in Figure 8.1a. The reservoir dimensions are $3000 \times 1500 \times 70 \mathrm{ft}$. Seven identical transverse hydraulic fractures with $300-\mathrm{ft}$ spacing are placed along a $2000-\mathrm{ft}$ horizontal well and there is no additional perforated interval along the well. The half-length, width, and permeability of each hydraulic fracture are $150 \mathrm{ft}, 0.025 \mathrm{ft}$, and 10 Darcies, respectively. The synthetic model resembles a densely-fractured, tight reservoir, which comprises 1819 small-scale natural fractures with various lengths and different orientations. The intensity of fractures varies in different regions. All other petrophysical parameters, fluid properties, and wellbore operating conditions are identical to the previous example.

The matrix grid is $157 \times 77 \times 1$ cells in $\mathrm{x}, \mathrm{y}$, and $\mathrm{z}$ directions, respectively. Table 8.3 describes the matrix grid used in the simulations of this example. There are 119 fracture control volumes, corresponding to seven hydraulic fractures, in the discrete-fracture domain, indicating that most gridblocks in this domain are treated as dead blocks in the simulations. Also, out of 12089 gridblocks in the continuum-fracture domain, 5771 cells do not contain any embedded natural fracture and are null blocks for flow simulations. Figure 8.5 shows four pressure profiles after three months, one year, three years, and six years of production for the reservoir shown in Figure 8.1a, which is the base case for the first example. The dark red areas denote original reservoir pressure and the dark blue 183 
represents wellbore bottomhole pressure. The pressures of hydraulic fractures are also mapped back to their original locations and depicted in the profiles. The impact of natural fracture network on the reservoir depletion is very clear in Figure 8.5. That is, regions with higher intensity of natural fractures experience faster pressure depletion. Furthermore, the pressure depletion pattern reflects the presence of locally-connected natural fracture networks close to the hydraulic fractures.

We repeated the simulation in the absence of numerous natural fractures to quantify their effect on production of unconventional resources. Figure 8.6 shows four pressure profiles in the absence of natural fracture network after three months, one year, three years, and six years of production, depicting significantly smaller drainage area compared to those shown in Figure 8.5. Even after six years, oil production is still limited to the proximity of the hydraulic fracture network. The profiles presented in Figure 8.6 are similar to those in previously published studies, which simulated unconventional reservoirs. The profiles shown in Figure 8.5 exhibit the novelty and applicability of the coupled model, developed in this study, for realistic simulation of fractured, low- permeability reservoirs.

Figure 8.7 compares oil production rate and average reservoir pressure calculated by the coupled model for both simulations, indicating much higher oil production and pressure depletion in the presence of the natural fracture network. Also, oil recovery after six years of production in the first simulation is about 2 times larger than that of the second simulation, emphasizing the noticeable contribution of pre-existing natural fractures on total oil production. The computational times for the first and second simulations were 67.6 and 2.6 minutes, respectively. 


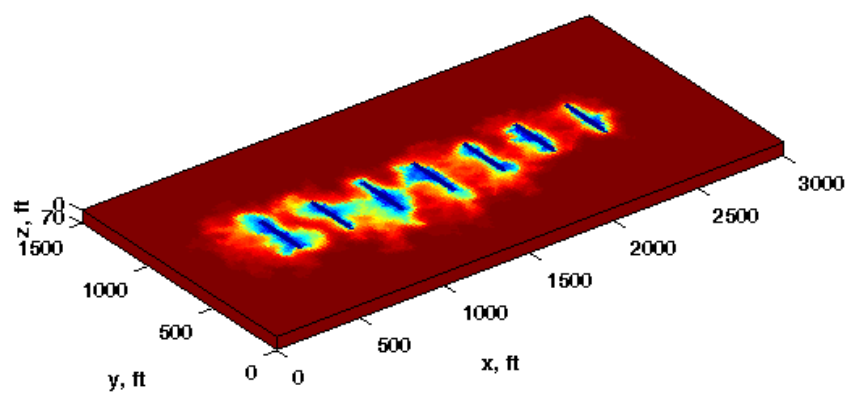

(a)

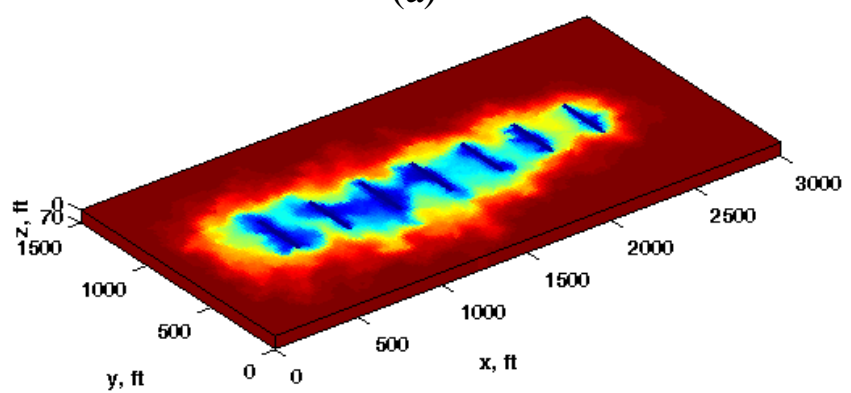

(b)

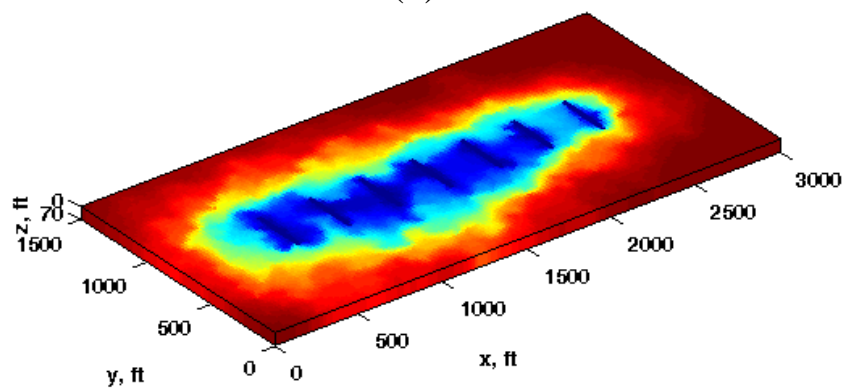

(c)

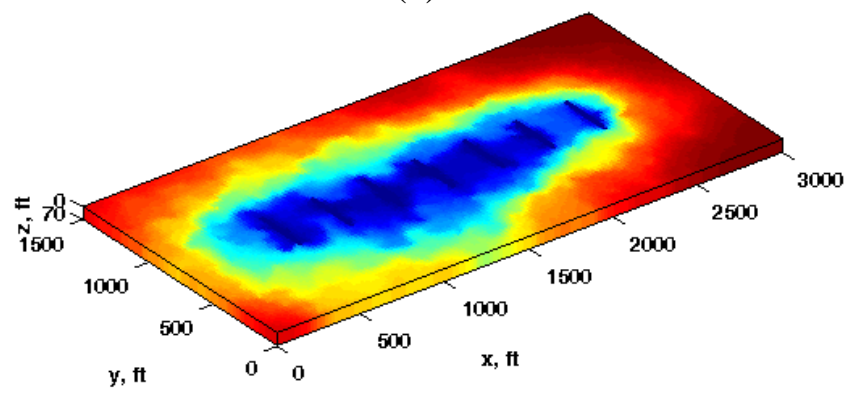

(d)

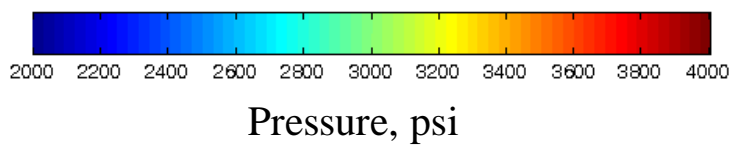

Figure 8.5: Pressure profiles simulated by the coupled model after (a) 3 months, (b) 1 year, (c) 3 years, and (d) 6 years of production for the reservoir shown in Figure 8.1a. 


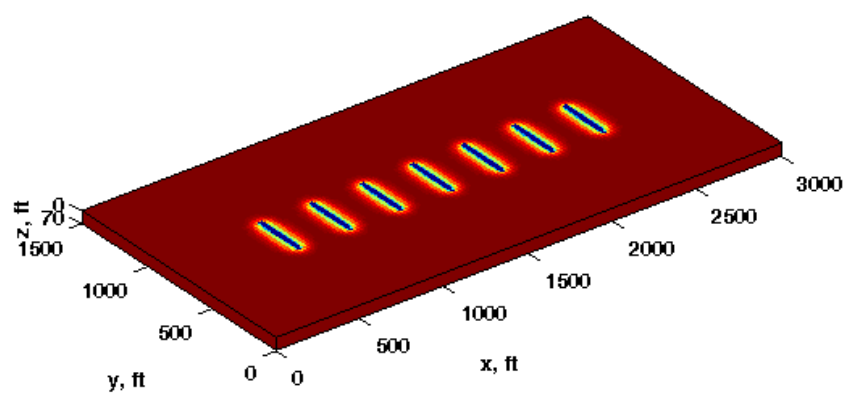

(a)

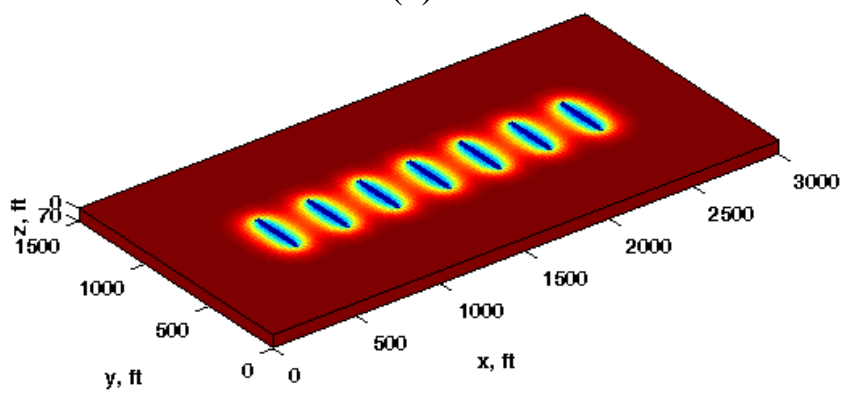

(b)

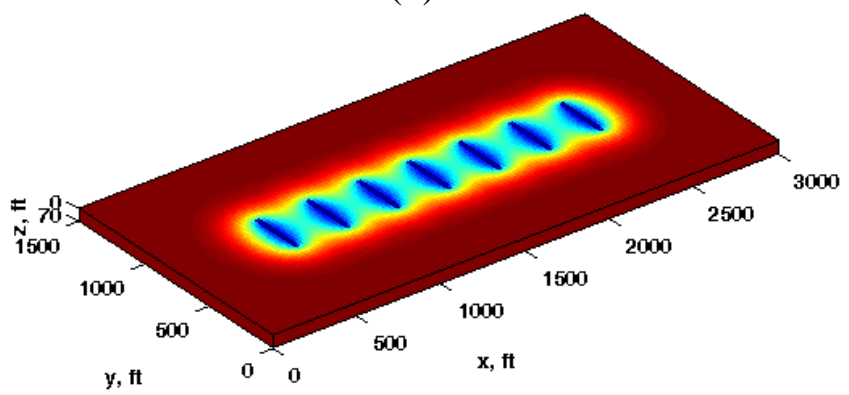

(c)

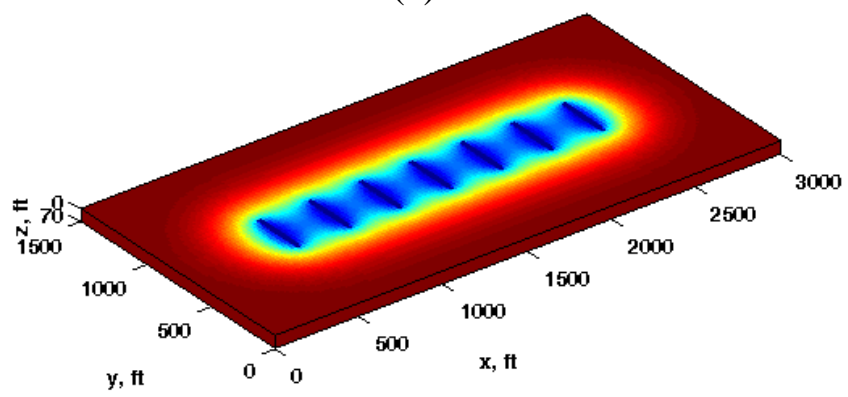

(d)

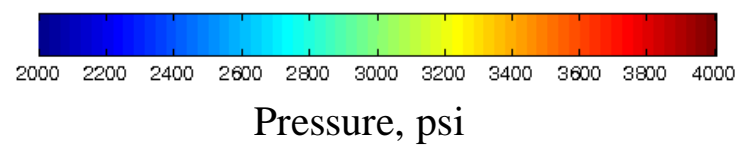

Figure 8.6: Pressure profiles simulated by EDFM after (a) 3 months, (b) 1 year, (c) 3 years, and (d) 6 years of production for the reservoir shown in Figure 8.1a but in the absence of the natural fracture network.

186 


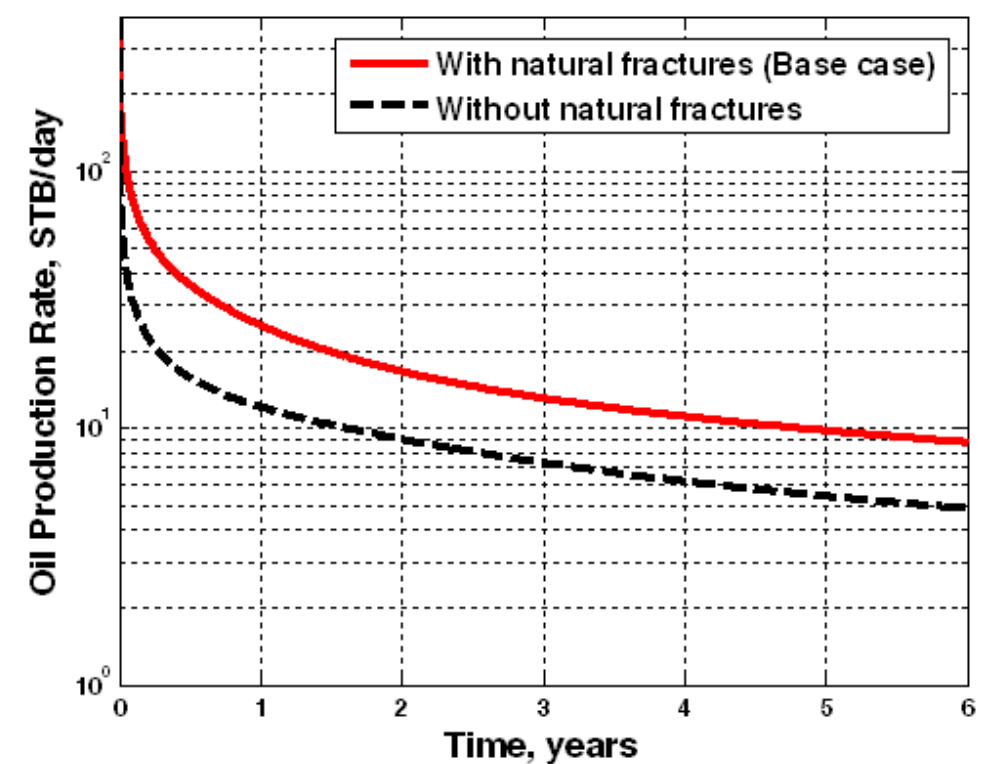

(a)

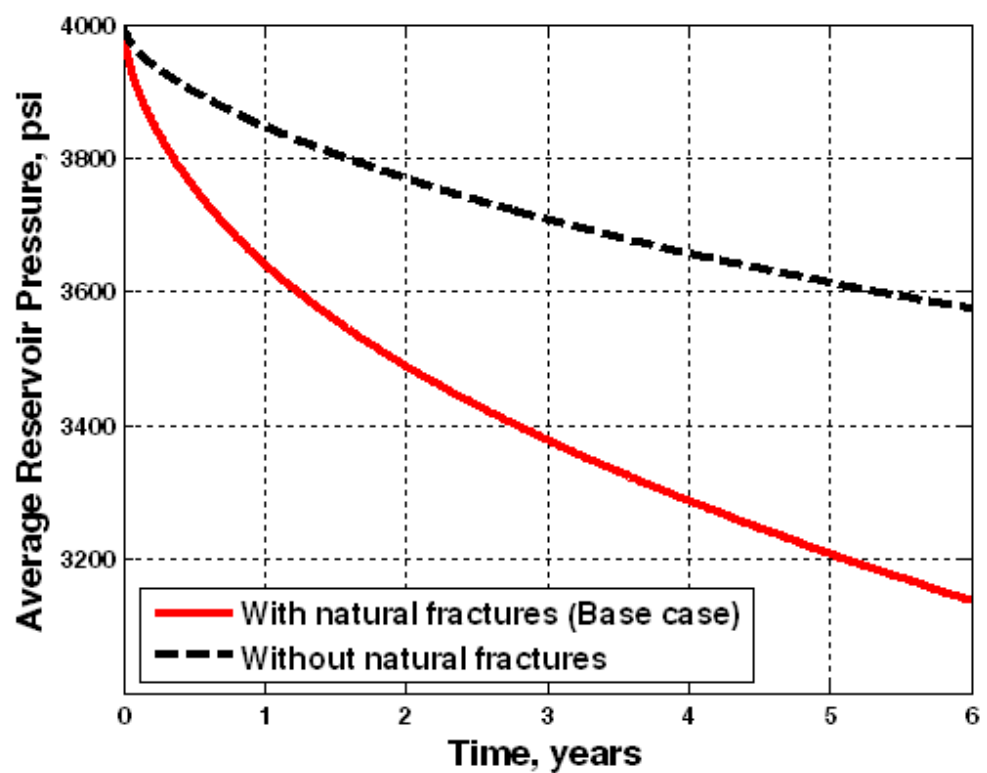

(b)

Figure 8.7: Comparison of (a) oil production rate, and (b) average reservoir pressure, over six years of production for the reservoir shown in Figure 8.1a, in the presence and absence of the natural fracture network. 
Additional simulations were made on the previous model reservoir to demonstrate the potential use of our coupled approach for optimizing fracture treatments and evaluating the efficiency of different completion schemes. We examine two additional stimulation schemes while retaining the network of natural fractures. Figure 8.8a shows the first scenario in which hydraulic fractures are longer and thinner compared to the base case. The width and half-length of seven hydraulic fractures are $0.015 \mathrm{ft}$ and $250 \mathrm{ft}$, respectively. Likewise, Figure 8.9a shows the second stimulation scheme in which ten hydraulic fractures with $200-\mathrm{ft}$ spacing are placed along the horizontal well. In this scenario, the width and half-length of hydraulic fractures are $0.0175 \mathrm{ft}$ and $150 \mathrm{ft}$, respectively. Thus, the total volume of hydraulic fractures is identical in all three scenarios.

The pressure profiles after three years of production are depicted in Figure $8.8 \mathrm{~b}$ and $8.9 \mathrm{~b}$ for both configurations. Likewise, Figure 8.10 compares oil production rate and also cumulative oil production for all three stimulation schemes. Despite its higher operational costs, creating ten hydraulic fractures is not the best stimulation strategy for maximizing production over six years for this model reservoir. However, the stimulation scenario with longer and thinner hydraulic fractures significantly improves total oil production after six years of production compared to the other two cases $(20.5 \%$ and 9.8\% higher oil recovery compared to the base and the last cases, respectively). These conclusions were made assuming that we want to maximize total production over six years. Based on different economic goals for field development, different decisions can be made for fracture treatments. The purpose of this example was to show how the coupled model can be used efficiently to improve stimulation designs and completion strategies. The computational times for simulating the model reservoirs shown in Figures 8.8a and $8.9 \mathrm{a}$ were 74.3 and 76.5 minutes, respectively. 


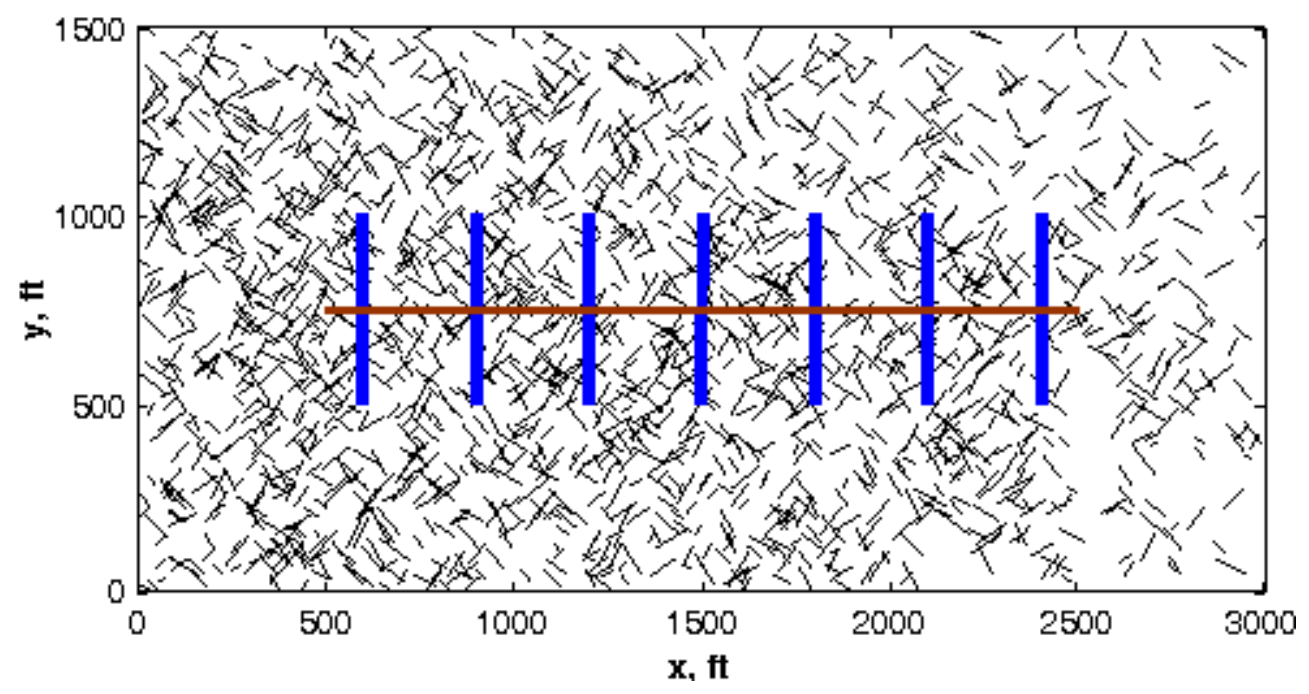

(a)

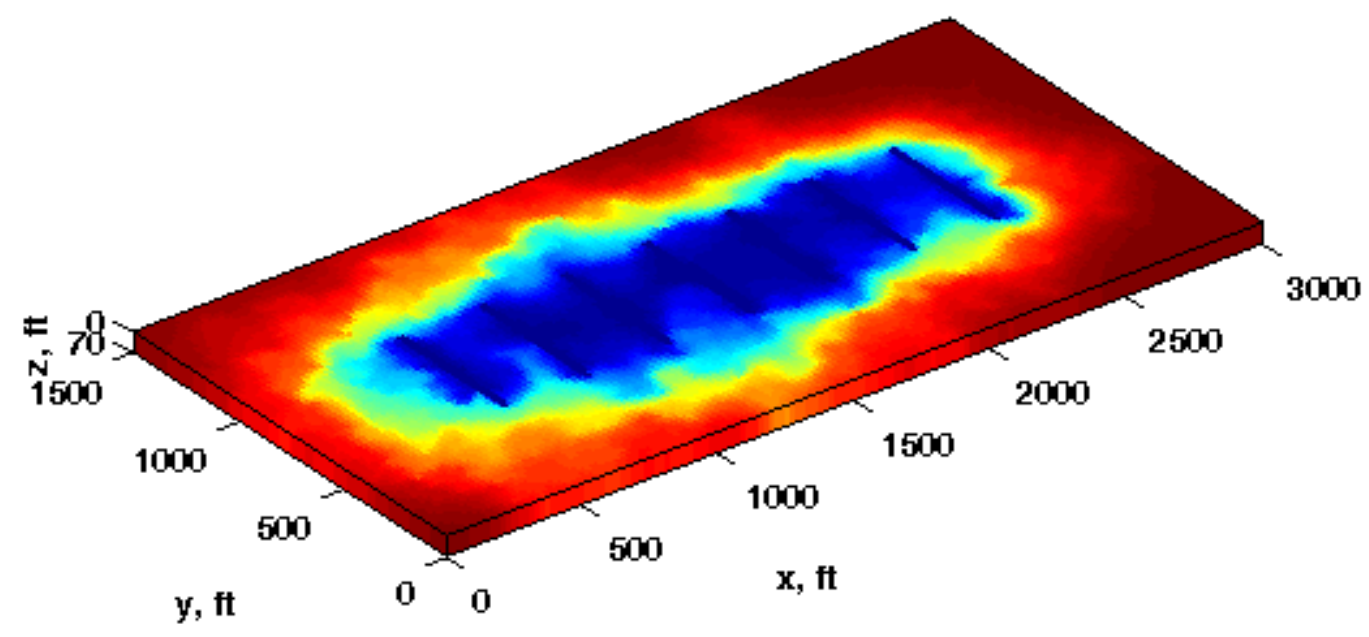

Pressure, psi

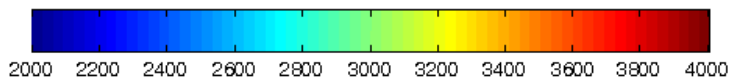

(b)

Figure 8.8: (a) A model reservoir which retains the natural fracture network in Figure 8.1a, but contains longer hydraulic fractures. (b) Pressure profile simulated by the coupled model after three years of production for the reservoir shown in Figure 8.8a. 


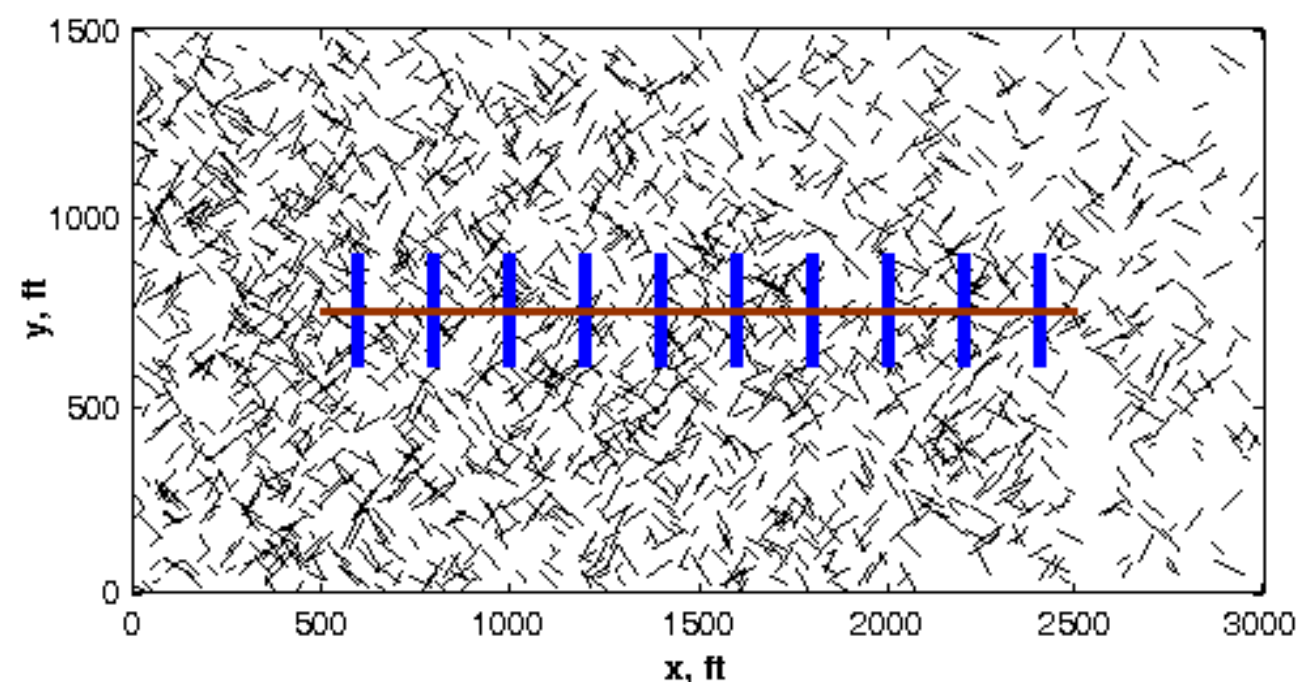

(a)

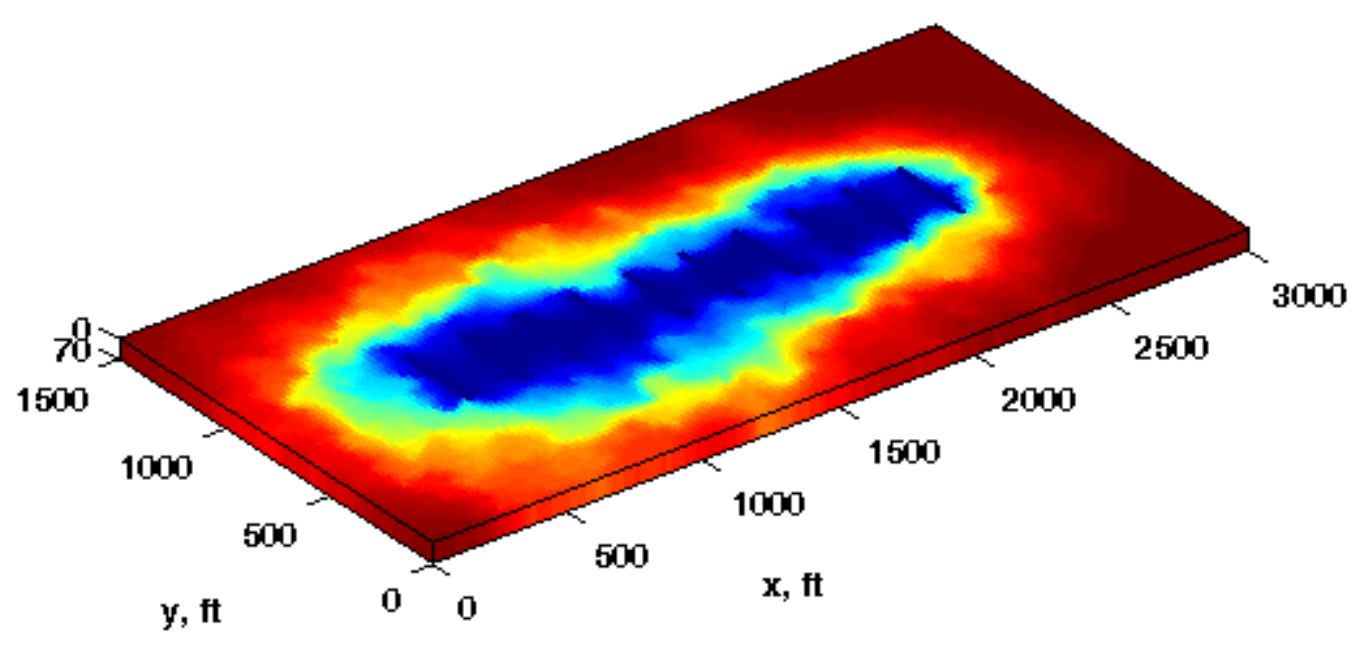

Pressure, psi

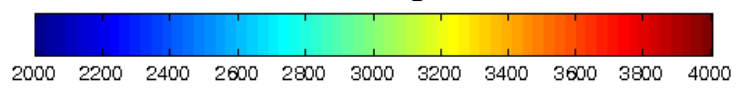

(b)

Figure 8.9: (a) A model reservoir which retains the natural fracture network in Figure 8.1a, but contains ten hydraulic fractures. (b) Pressure profile simulated by the coupled model after three years of production for the reservoir shown in Figure 8.9a. 


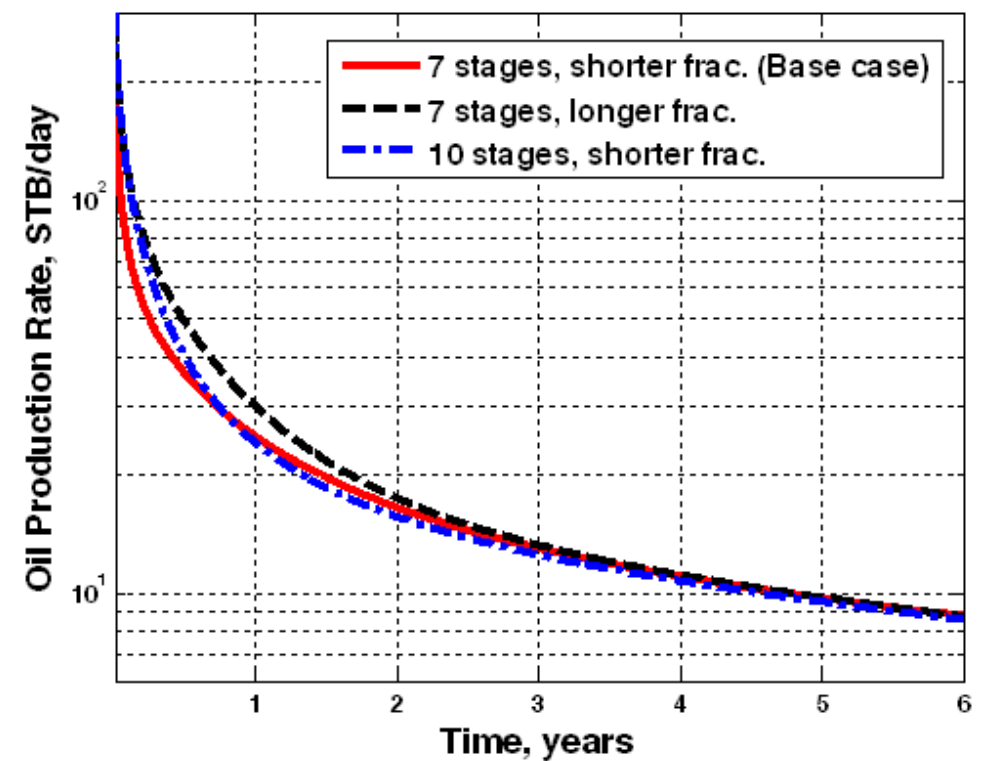

(a)

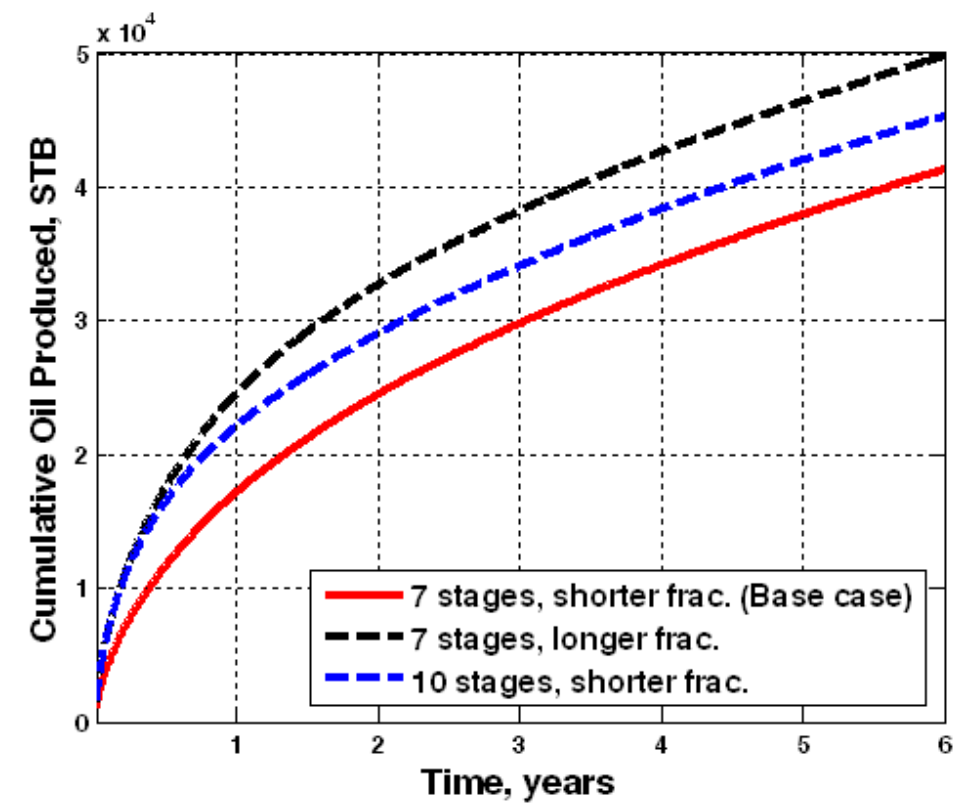

(b)

Figure 8.10: Comparison of (a) oil production rate, and (b) cumulative oil production, over six years of production for the reservoir models shown in Figures 8.1a, 8.8a, and 8.9a. 
As previously mentioned, hydraulic fracture complexity is a common occurrence for unconventional reservoirs. Since we use the EDFM approach for modeling hydraulic fractures, we can solve for complex hydraulic fracture patterns as well as simple fracture geometries. Thus, the next simulation scenario presented retains the same natural fracture network as the other cases but includes a multi-stranded, complex hydraulic fracture pattern, as depicted in Figure 8.11a. This hydraulic fracture geometry resembles a more realistic hydraulic fracture pattern observed in unconventional reservoirs, as schematically depicted in Figure $8.11 \mathrm{~b}$. All parameters for the simulation except the fracture half-length are identical to the base case.

Figure 8.12 shows pressure profiles after six months and three years of production. The first and second rows correspond to the simulations in the absence and presence of the natural fracture network, respectively. The pressure maps, especially those simulated in the absence of natural fractures, clearly illustrate that pressure depletion is primarily limited to the reservoir area directly adjacent to the hydraulic fracture network. The computational time for simulating the model reservoir shown in Figure 8.11a was 82.3 minutes.

The pressure maps exhibit the applicability of the EDFM approach for studying production performance of horizontal wells with complex hydraulic fracture patterns. Capturing the effect of this complexity can be very important in evaluating the performance of multi-lateral wells. There has been a considerable debate on the desirability of complex fracture growth in low-permeability reservoirs and our modeling approach can be used efficiently to shed light on this problem. 


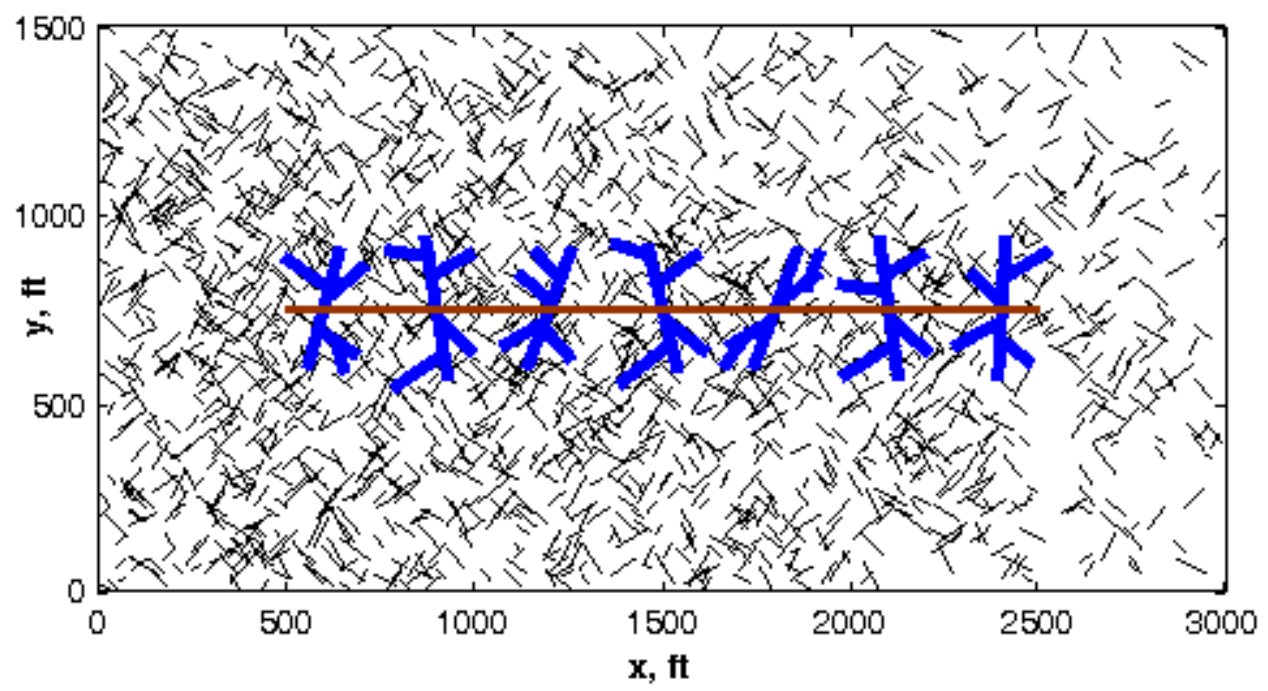

(a)

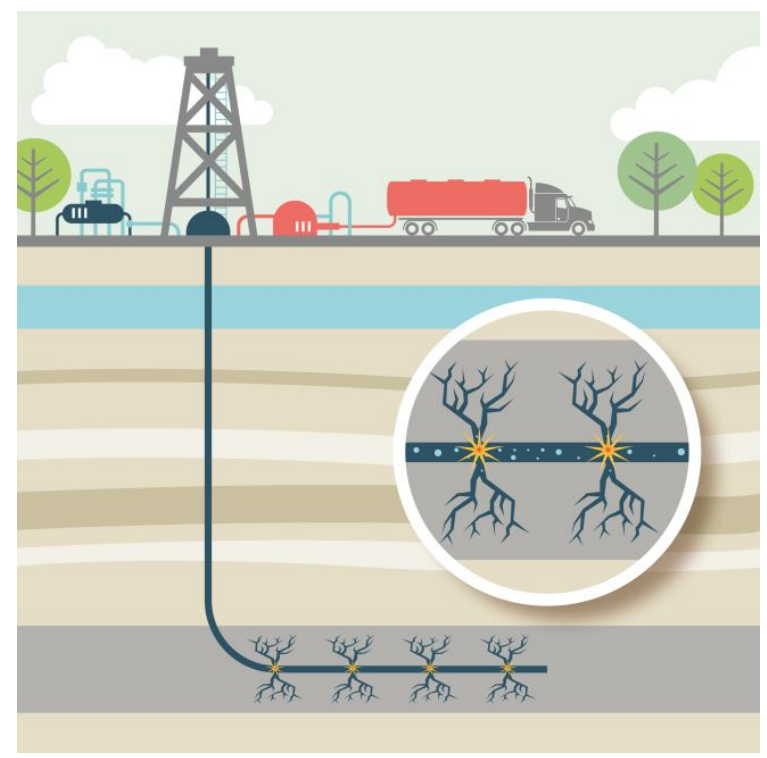

(b)

Figure 8.11: (a) A model reservoir which retains the natural fracture network in Figure 8.1a, but comprises a multi-stranded complex hydraulic fracture pattern. (b) Schematic geometry of hydraulic fracture propagation in unconventional reservoirs (from the website of University of Michigan). 

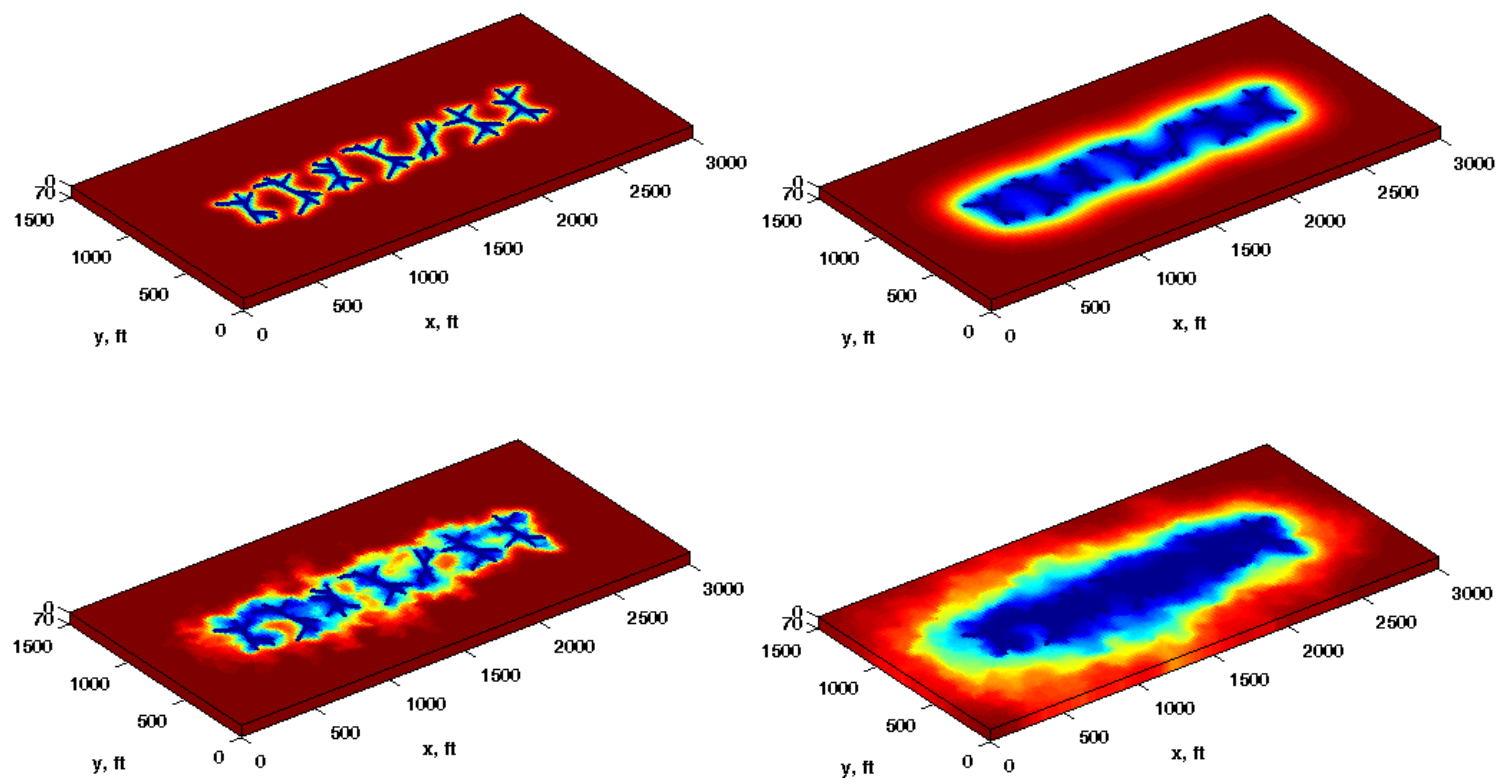

Pressure, psi

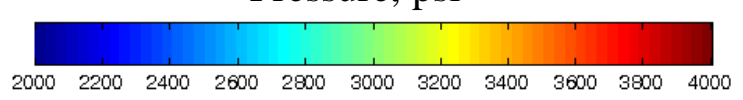

(a)

(b)

Figure 8.12: Pressure profiles after (a) 3 months, and (b) 3 years of production for the model reservoir shown in Figure 8.11a. The first and second rows show the pressure maps simulated in the absence and presence of the natural fracture network, respectively.

\begin{tabular}{|c|c|c|}
\hline Reservoir model & Grid size & \multicolumn{1}{|c|}{ Grid description } \\
\hline $\begin{array}{c}\text { Figures 8.1a, 8.8a, } \\
\text { and 8.11a }\end{array}$ & $157 \times 77 \times 1$ & $\Delta \mathrm{x}(\mathrm{ft}): 29 \times 20,6 *(16,8,16,13 \times 20), 16,8,16,29 \times 20$ \\
\cline { 3 - 3 } & & $\Delta \mathrm{y}(\mathrm{ft}): 37 \times 20,8,6,8,37 \times 20$ \\
\hline \multirow{2}{*}{ Figure 8.9a } & $160 \times 77 \times 1$ & $\Delta \mathrm{x}(\mathrm{ft}): 29 \times 20,9 *(16,8,16,8 \times 20), 16,8,16,29 \times 20$ \\
\cline { 3 - 3 } & & $\Delta \mathrm{y}(\mathrm{ft}): 37 \times 20,8,6,8,37 \times 20$ \\
\hline
\end{tabular}

Table 8.3: Grid description for various simulations performed in Section 8.4.1. 


\subsubsection{D Unconventional Gas Reservoir}

Hydraulic fracture planes are not always vertical, especially when fracturing fluid opens up non-vertical natural fractures during stimulation treatment. Here, we simulate natural gas production from a synthetic 3D tight reservoir model shown in Figure 8.13. The reservoir dimensions are $2000 \times 1000 \times 100 \mathrm{ft}$. The model contains three hydraulic fractures, represented by blue planes in Figure 8.13, with 500-ft spacing. The left and middle hydraulic fractures are inclined $60^{\circ}$ and $75^{\circ}$, respectively, from the horizontal plane while the right hydraulic fracture is vertical. Furthermore, the hydraulic fractures partially penetrate the height of the formation. That is, the height of hydraulic fractures is $60 \mathrm{ft}$, which penetrates from $20-\mathrm{ft}$ to $80-\mathrm{ft}$ in the $\mathrm{z}$ direction. The width and permeability of each hydraulic fracture are $0.01 \mathrm{ft}$ and 5 Darcies, respectively. The formation porosity, horizontal permeability, vertical permeability, temperature, and the rock compressibility are $0.05,100 \mathrm{nd}, 10 \mathrm{nd}, 120^{\circ} \mathrm{F}$, and $8 \times 10^{-6} \mathrm{psi}^{-1}$, respectively. The water saturation in the rock matrix is equal to the irreducible water saturation of 0.2 and the endpoint relative permeability of gas is 0.75 . Furthermore, the reservoir comprises 1892 small-scale natural fractures, presented by red planes in Figure 8.13. The aperture, permeability, and height of all natural fractures are $0.002 \mathrm{ft}, 500 \mathrm{md}$, and $20 \mathrm{ft}$, respectively. The initial reservoir pressure is $4500 \mathrm{psi}$ and the horizontal well produces at a constant bottomhole pressure of 1500 psi. Table 8.4 summarizes the properties used in the simulations of this section.

The matrix grid is $83 \times 41 \times 5$ cells in $\mathrm{x}, \mathrm{y}$, and $\mathrm{z}$ directions, respectively. The description of matrix grid is presented in Table 8.5. The cell dimensions are $20 \mathrm{ft}$ in the $\mathrm{z}$ direction, indicating that hydraulic fractures penetrate three middle computational layers and do not penetrate the first and last ones. Figure 8.14 shows the pressure profiles, computed by the coupled model, after ten years of gas production for the first, second, fourth, and fifth computational layers. Although none of hydraulic fractures penetrate the 
first and last layers, a considerable amount of pressure depletion is observed in both layers because of vertical proximity to the hydraulic fractures and their neighboring depleted areas. Moreover, due to presence of randomly-generated natural fractures, the pressure depletion patterns are different in various layers. We also repeated the simulation assuming that hydraulic fractures fully penetrate the formation height. Figure 8.15 compares the calculated gas production rate for both configurations (partiallypenetrating and fully-penetrating). The total gas production after ten years is $248 \mathrm{MMscf}$ and $337 \mathrm{MMscf}$ for partially-penetrating and fully-penetrating fractures, respectively. Consequently, $26.4 \%$ of total gas production is lost due to inefficient fracturing treatment. The computational times for the first and second simulations were 130.1 and 112.5 minutes, respectively.

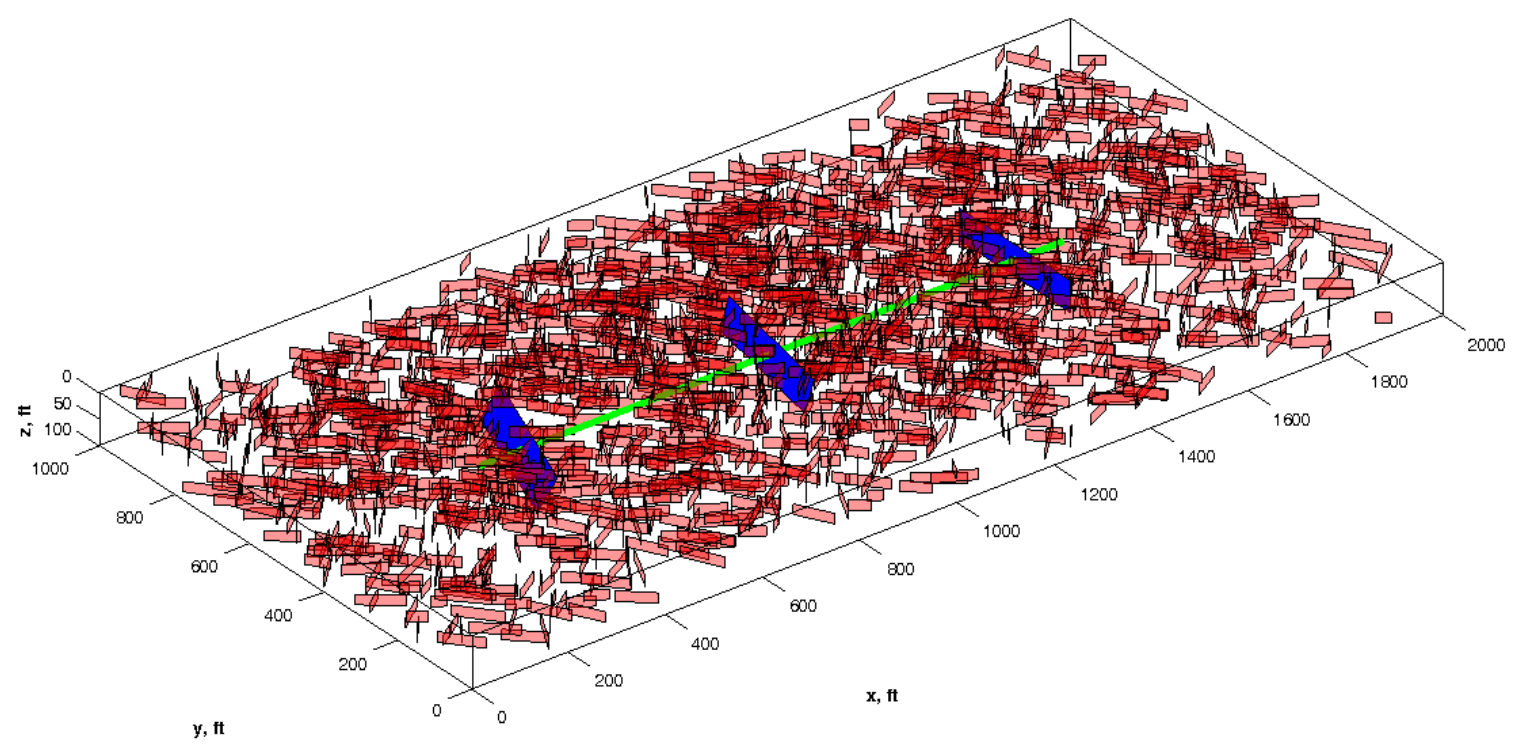

Figure 8.13: A synthetic 3D reservoir studied in Section 8.4.2. The model comprises a 1200-ft horizontal well (green line), 3 hydraulic fractures (blue planes), and 1892 small-scale natural fractures (red planes). 


\begin{tabular}{|c|c|c|c|}
\hline Variable & Value & Variable & Value \\
\hline Matrix porosity & 0.05 & Hydraulic fracture width & $0.01 \mathrm{ft}$ \\
\hline Matrix horizontal permeability & $100 \mathrm{nd}$ & Hydraulic fracture & $5 \mathrm{D}$ \\
\hline Matrix vertical permeability & $10 \mathrm{nd}$ & Natural fracture aperture & $0.002 \mathrm{ft}$ \\
\hline Reservoir temperature & $120^{\circ} \mathrm{F}$ & Natural fracture & $500 \mathrm{md}$ \\
\hline Rock compressibility & $8 \times 10^{-6} \mathrm{psi}^{-1}$ & Initial water saturation & 0.2 \\
\hline Initial reservoir Pressure & $4500 \mathrm{psi}$ & Producer BHP & $1500 \mathrm{psi}$ \\
\hline \multicolumn{4}{|c|}{ Component : $\mathrm{CH}_{4}$} \\
\hline Molecular weight (lb/lb-mol) & 16.0 & Critical temperature $\left({ }^{\circ} \mathrm{R}\right)$ & 343.0 \\
\hline Acentric factor & 0.013 & Critical pressure (psia) & 667.8 \\
\hline Parachor & 71.0 & Critical volume $\left(\mathrm{ft}^{3} / \mathrm{lb}-\mathrm{mol}\right)$ & 1.6 \\
\hline
\end{tabular}

Table 8.4: Summary of properties used in the simulations performed for the model reservoir shown in Figure 8.13.

\begin{tabular}{|c|l|l|}
\hline Reservoir model & Grid size & \multicolumn{1}{c|}{ Grid description } \\
\hline \multirow{2}{*}{ Figure 8.13 } & \multirow{3}{*}{$83 \times 41 \times 5$} & $\Delta \mathrm{x}(\mathrm{ft}): 19 \times 25,2 *(20,10,20,18 \times 25), 20,10,20,19 \times 25$ \\
\cline { 3 - 3 } & & $\Delta \mathrm{y}(\mathrm{ft}): 19 \times 25,20,10,20,19 \times 25$ \\
\cline { 3 - 3 } & & $\Delta \mathrm{z}(\mathrm{ft}): 5 \times 20$ \\
\hline
\end{tabular}

Table 8.5: Grid description for two simulations performed in Section 8.4.2. 


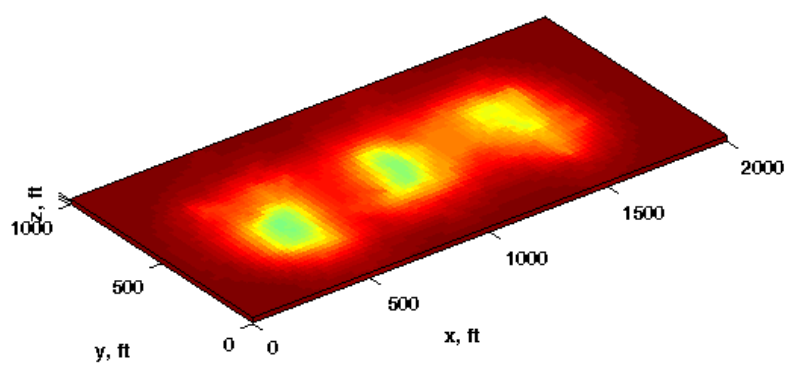

(a)

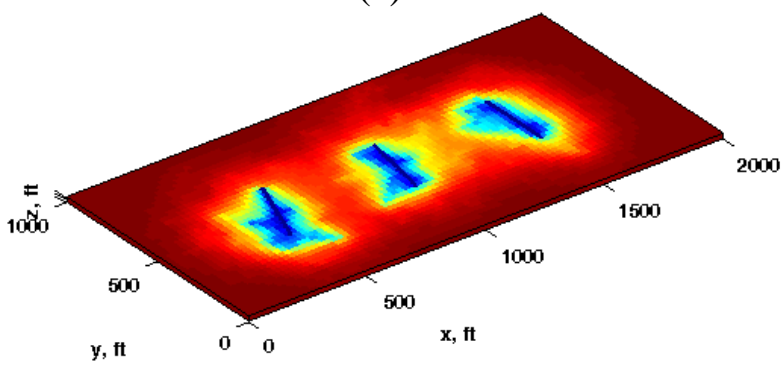

(b)

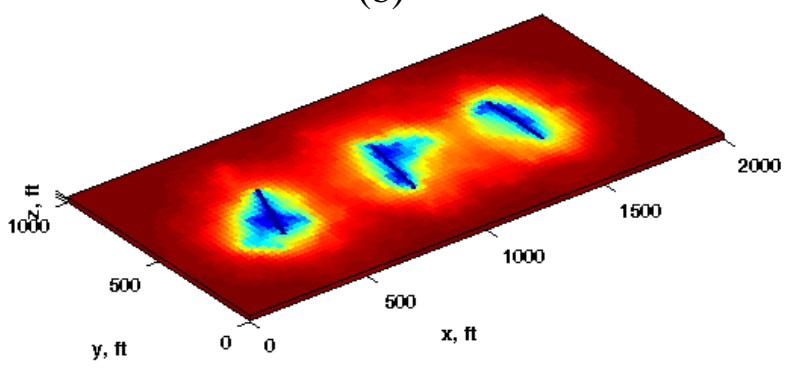

(c)

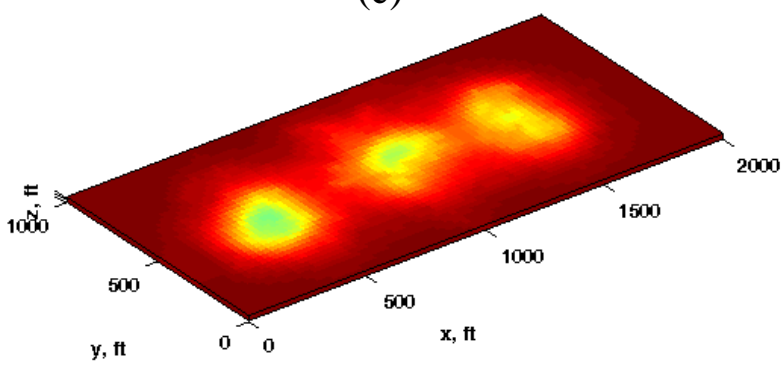

(d)

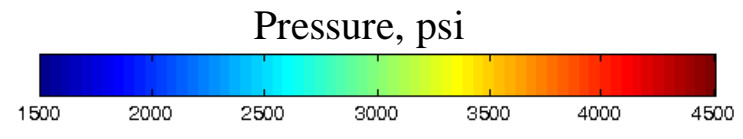

Figure 8.14: Pressure profiles simulated by the coupled model after 10 years of production in the (a) first, (b) second, (c) fourth, and (d) fifth computational layers of the model reservoir shown in Figure 8.13.

198 


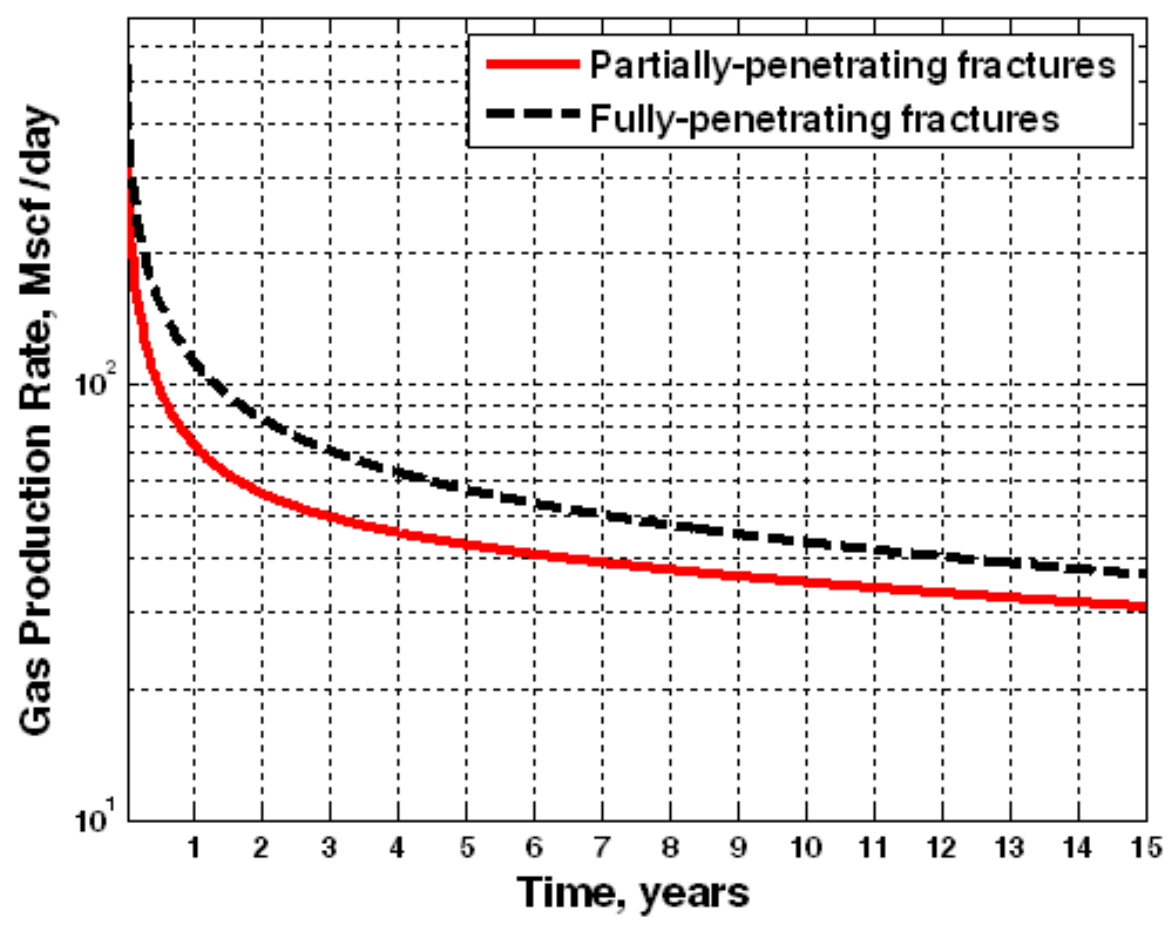

Figure 8.15: Gas production rate calculated by the coupled model over ten years of production for the reservoir shown in Figure 8.13 wherein all hydraulic fractures penetrate $60 \%$ of the formation height, compared with the one calculated for the same reservoir where all hydraulic fractures fully penetrate the formation height. 


\subsection{DiscuSSION}

In this chapter, we proposed a hybrid method for the simulation of unconventional reservoirs in the presence of a complex hydraulic fracture network and numerous smallscale natural fractures. The developed approach is designed to precisely and efficiently reflect the effect of both hydraulic and natural fractures. However, accurate and reliable simulation of unconventional reservoirs requires incorporating all physics, which have considerable impact on fluid flow in these reservoirs. For instance, Javadpour et al. (2007) discussed the contribution of gas desorption to gas flow in shale plays. Rubin (2010) presented example simulations to quantify the effect of non-Darcy flow in shale gas reservoirs. Cipolla et al. (2010) incorporated the effect of stress-dependent fracture conductivity in reservoir simulations and showed that the well productivity can be significantly reduced in unconventional reservoirs. It should be noted that different physics mentioned above were neglected in the example simulations of this chapter, but all can be added to the hybrid model in order to develop a complete and robust unconventional reservoir simulator.

The coupled dual continuum and discrete fracture model can be used not only for the simulation of unconventional reservoirs but also for simulating naturally fractured reservoirs. Natural fracture systems commonly show an asymmetrical distribution of fracture sizes, with numerous small fractures and few large fractures. As previously mentioned, Lee et al. (2001) presented a hierarchical modeling approach for addressing fluid flow in naturally fractured reservoirs. In this approach, small fractures are represented by their aggregate effective properties and the large-scale fractures are modeled explicitly. An appropriate alternative for this approach is the hybrid method presented in this chapter. That is, small fractures are modeled using the proposed dual continuum approach and large-scale fractures are modeled explicitly using EDFM. 


\subsection{Highlights of THE CHAPTER}

- A coupled dual continuum and discrete fracture model is developed to simulate production from unconventional reservoirs.

- Large-scale hydraulic fractures (macro-fractures) are modeled explicitly using the EDFM approach. EDFM can handle the complexity of hydraulic fracture networks, which is a common occurrence in low-permeability reservoirs.

- Numerous small-scale natural fractures (micro-fractures) are modeled using a dual continuum approach. The parameters for dual continuum modeling of numerous natural fractures are derived by upscaling the EDFM equations.

- The coupled model is similar to a triple porosity approach, including three domains: matrix, discrete-fracture, and continuum-fracture domains. A systematic approach is devised to calculate transport parameters between all three domains.

- Comparison of the results using the coupled dual continuum and EDFM approach with that of using the EDFM approach to represent all natural and hydraulic fractures explicitly shows that reasonably accurate results can be obtained at much lower computational time by using the coupled approach with moderate grid refinements.

- Several examples are presented to show the applicability, robustness, and performance of the hybrid method for the simulation of unconventional reservoirs in the presence of complex hydraulic fracture pattern and numerous pre-existing fractures.

- The coupled model can be used efficiently to improve stimulation designs and completion strategies.

- An underlying network of natural fractures is very important to recovery. 


\section{Chapter 9: Summary, Conclusions, and Recommendations}

\subsection{SUMMARY AND CONCLUSIONS}

The following presents the summary and conclusions for this research:

1. We developed an embedded discrete fracture model (EDFM) for our in-house fully-implicit compositional reservoir simulator (GPAS). The work performed in this study is an extension of an algorithm developed by Li and Lee (2008) for modeling fluid flow in vertical fractures. The extended algorithm includes slanted fractures of any orientation, honoring the complexity and heterogeneity of a typical fractured reservoir. EDFM borrows the dual medium concept from dual continuum models, but also incorporates the effect of each fracture explicitly. EDFM employs a structured grid to surmount challenges associated with unstructured gridding.

2. EDFM is based on non-neighboring connections (NNC). We introduced and described three types of NNCs required for modeling vertical and slanted fractures. It is necessary to properly calculate the transmissibility between NNCs using the equations presented in this work.

3. We added the NNC term to the mass balance equations in the EOS compositional module of GPAS. EDFM is compatible with reservoir simulators that allow for NNCs and transmissibility modifiers (such as commercial reservoir simulators).

4. We developed a pre-processing code to provide the required data for fluidflow simulations in GPAS using the EDFM approach. The input of the pre- 
processing code is the description of model reservoir, including the reservoir dimensions, fracture network, location of wells, structured grid for the matrix domain, aperture and permeability of fractures, and porosity and permeability of matrix. Subsequently, the parameters calculated in the pre-processing code include porosity, permeability, and depth of fracture control volumes, transmissibility between NNCs, transmissibility between adjacent fracture cells, and well indices for the fractures intercepted by a well.

5. The accuracy of the EDFM approach for modeling primary production from multiply-fractured horizontal wells was confirmed by its match with the analytical solutions for two production schemes, constant-pressure production and constant-rate production.

6. The accuracy of the EDFM approach was confirmed by comparing the EDFM results with the fine-grid, explicit-fracture simulations for case studies including isolated fractures, intersected orthogonal fractures, and a nonorthogonal non-aligned fracture. The case studies examined both waterflooding and compositional gas injection.

7. We presented examples of water-flooding, compositional gas injection, and primary depletion to demonstrate the applicability, robustness, and efficiency of the EDFM approach for modeling fluid flow in naturally fractured reservoirs.

8. EDFM offers a computationally-efficient approach for simulating fluid flow in fractured reservoirs. Comparison of our results using the EDFM approach with fine-grid simulations showed that accurate results can be achieved with moderate grid refinements. This was further verified in our mesh sensitivity 
study that the EDFM approach using moderate grid refinement can obtain a converged solution.

9. The EDFM approach was found to be insensitive to grid orientation.

10. We compared the EDFM approach to an unstructured discrete fracture model (USDFM) and a dual permeability method for simulating multiphase flow in NFRs. Simulations showed consistency and agreement of results using the EDFM and USDFM methods in synthetic models with complex fracture patterns. Simulations also showed that the dual permeability model fails to provide accurate solutions in the presence of large-scale fractures and high localized anisotropy.

11. We incorporated the dynamic behavior of fractures into EDFM. The coupled approach allows the impact of stress regime on fluid flow in a 3D discrete fracture network. The non-linear Barton-Bandis joint model was used to represent normal deformation of pre-existing natural fractures. Also, fractureconductivity tables were used to model dynamic behavior of propped hydraulic fractures in stimulated reservoirs.

12. Using the Barton-Bandis joint model, fractures under lower initial effective normal stress were found prone to larger aperture decline and permeability loss with pressure depletion.

13. Consideration of dynamic behavior of fractures substantially affects pressure depletion and hydrocarbon recovery. The significance of such effects on production strongly depends on parameters controlling the deformation behavior of fractures. 
14. Based on examples presented in this research, implementation of fracture geomechanical effects in EDFM did not degrade the computational performance of EDFM.

15. We developed a coupled dual continuum and discrete fracture model to simulate production from unconventional reservoirs. Large-scale hydraulic fractures (macro-fractures) are modeled explicitly using the EDFM approach and numerous small-scale natural fractures (micro-fractures) are modeled using a dual continuum approach. The coupled model is similar to a triple porosity approach, including three domains: matrix, discrete-fracture, and continuum-fracture domains.

16. EDFM can handle the complexity of hydraulic fracture networks, which is a common occurrence in unconventional reservoirs.

17. The transport parameters for dual continuum modeling of numerous natural fractures were derived by upscaling the EDFM equations.

18. Comparison of the results using the coupled dual continuum and EDFM approach with that of using the EDFM approach to represent all natural and hydraulic fractures explicitly showed that reasonably accurate results can be obtained at much lower computational cost by using the coupled approach with moderate grid refinements.

19. We presented several examples to show the applicability and performance of the hybrid method for the simulation of unconventional reservoirs. An underlying network of natural fractures was found very important to recovery.

20. We examined multi-stage hydraulic fractures with multiple configurations to show how the coupled model can be used efficiently to improve stimulation designs and completion strategies. 


\subsection{RECOMMENDATIONS FOR FUTURE RESEARCH}

The following recommendations are suggested for future studies:

1. As repeatedly emphasized in this research, one advantage of the EDFM approach compared to other DFMs is its compatibility with existing finite difference reservoir simulators. Hence, we recommend implementing the EDFM approach in two other University of Texas compositional reservoir simulators, UTCOMP and UTCHEM. UTCOMP is a 3D compositional reservoir simulator capable of simulating a variety of enhanced oil recovery methods (Chang, 1990). UTCOMP has several numerical and physical features such as higher-order finite difference methods, full physicaldispersion tensor, and gas-foam flooding. Likewise, UTCHEM is a 3D compositional reservoir simulator developed for modeling chemical enhanced oil recovery methods (Delshad et al., 1996). In order to solve the governing equations, both simulators employ an IMPEC scheme that refers to a formulation in which pressure is solved implicitly and concentrations and saturations are solved explicitly. Implementing the EDFM approach in UTCOMP and UTCHEM provides an effective and reliable environment to study a variety of enhanced oil recovery processes in naturally fractured reservoirs. Furthermore, Varavei (2009) developed a thermal model in GPAS; we also recommend coupling the EDFM approach with this model in order to study thermal enhanced oil recovery methods in fractured systems.

2. The pre-processing code for the EDFM approach was developed in the MATLAB environment. For the sake of compatibility with existing reservoir simulators developed at The University of Texas at Austin (GPAS, UTCOMP, and UTCHEM), we recommend translating this code to FORTRAN. This is 
especially needed for applications where the geometry of fracture network changes during simulation, and hence the pre-processing code needs to be used in a dynamic manner.

3. Investigate the accuracy of the EDFM approach for modeling fluid flow in synthetic models with complex fracture patterns by comparing its results with fine-grid unstructured discrete fracture simulations that consider slanted fractures.

4. Investigate the accuracy of the EDFM approach when dispersion term is included in the simulations.

5. Evaluate the accuracy of the coupled geomechanics and fluid-flow simulations presented in this work using the results of a fully-coupled geomechanics and fluid-flow model.

6. Incorporate all physics, which have considerable impact on fluid flow in tight oil and shale gas reservoirs, into the hybrid model presented in this work in order to develop a complete and robust unconventional reservoir simulator.

7. Apply the EDFM approach in modeling contaminant transport in naturally fractured aquifers.

8. Investigate using higher-order discretization schemes for application in matrix and fracture domains. 


\section{Appendix A: Sample Input Files}

Appendix A presents examples of GPAS input files used in this research. The first and the second input files correspond to the case studies described in Sections 5.2.2 and 8.4.2, respectively.

\section{A.1 Compositional Gas InJECTION USING EDFM (SECTION 5.2.2)}

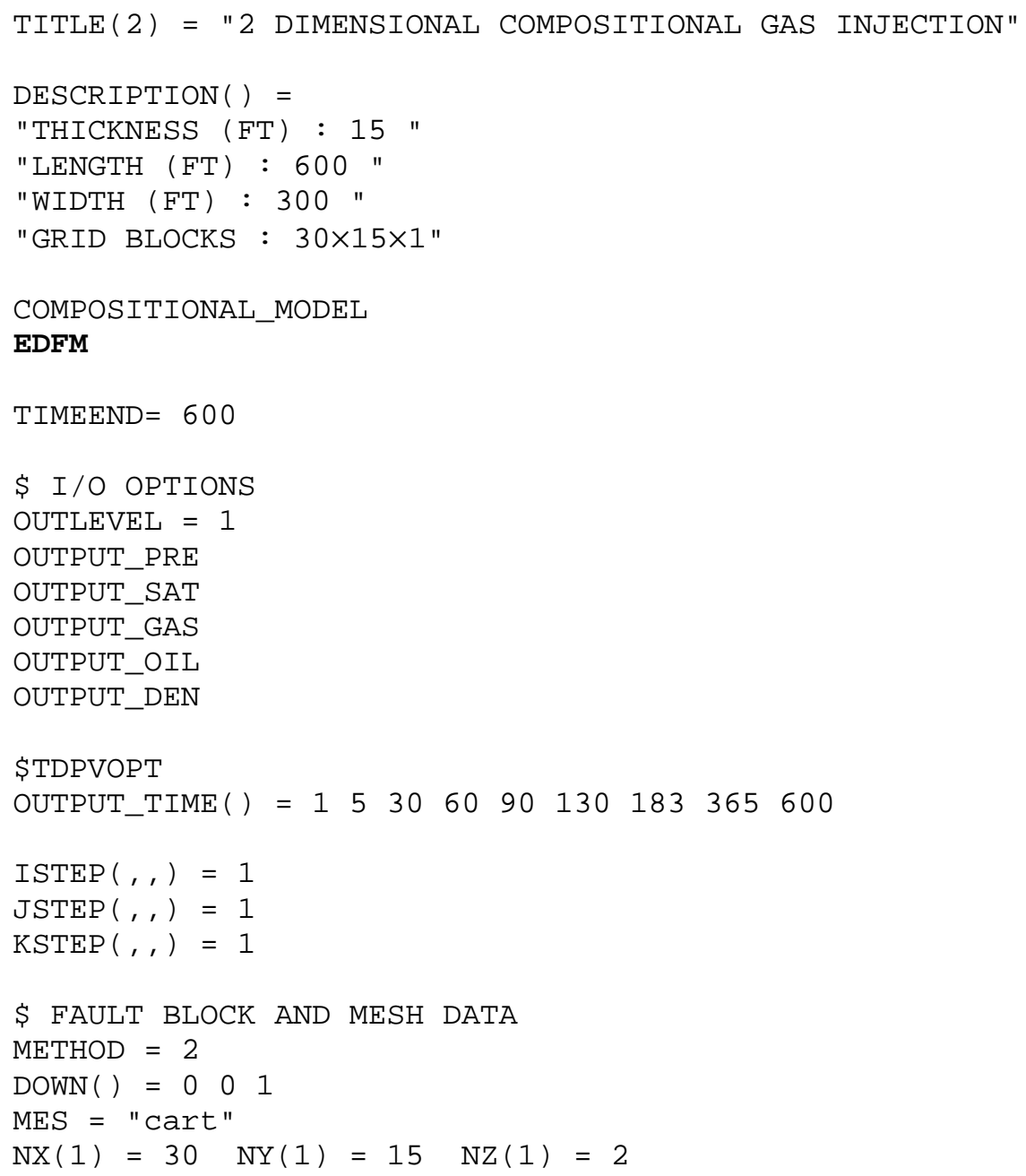




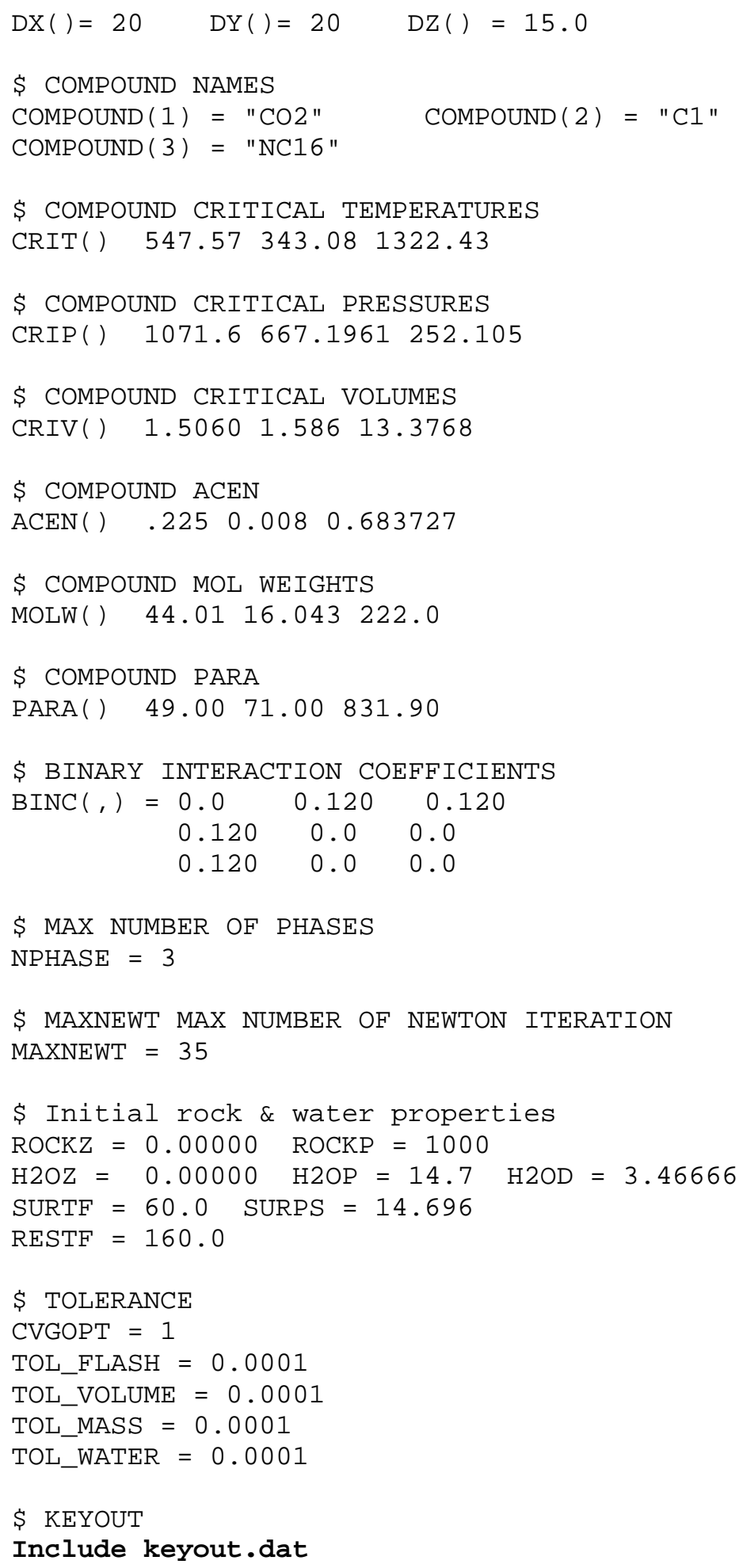




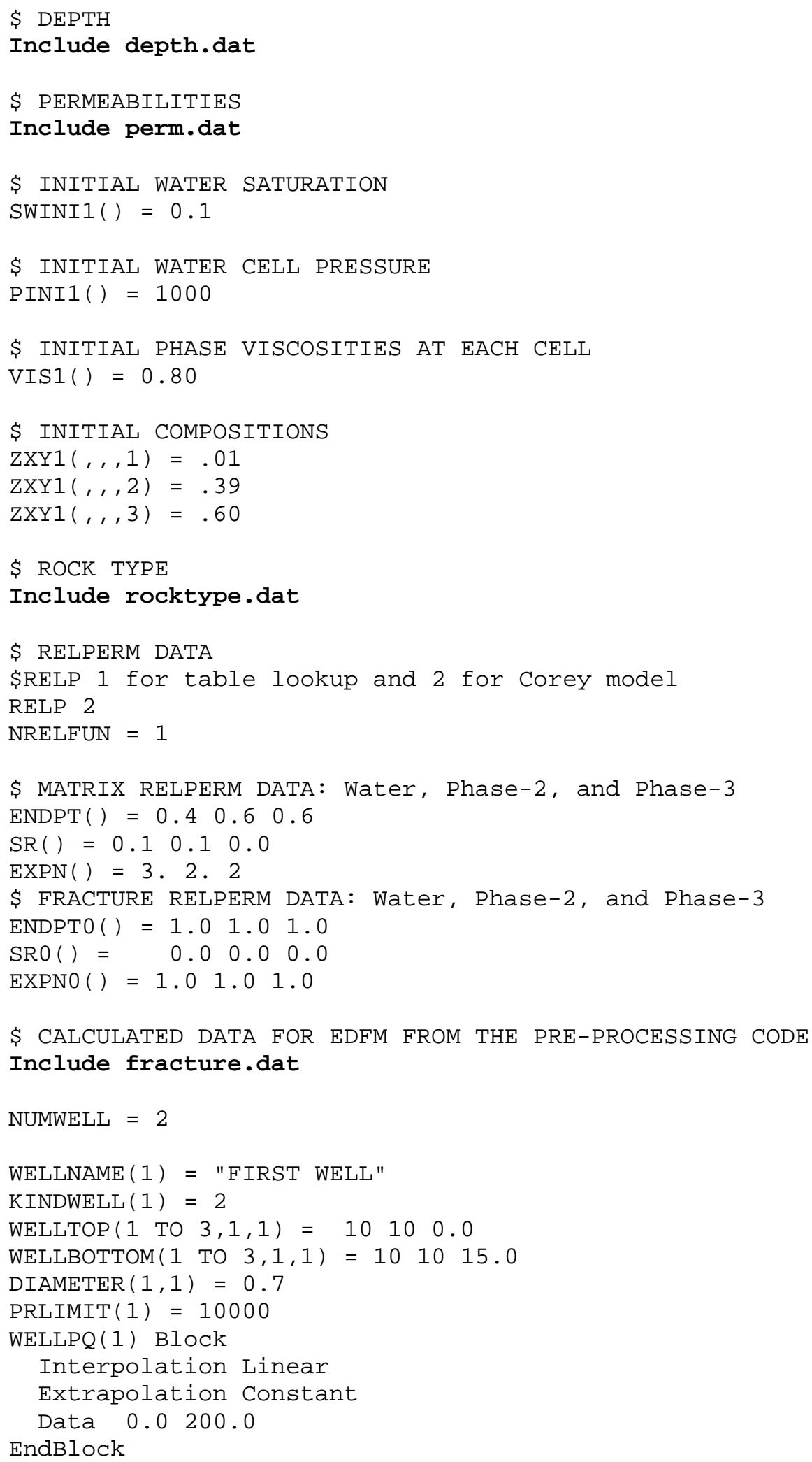




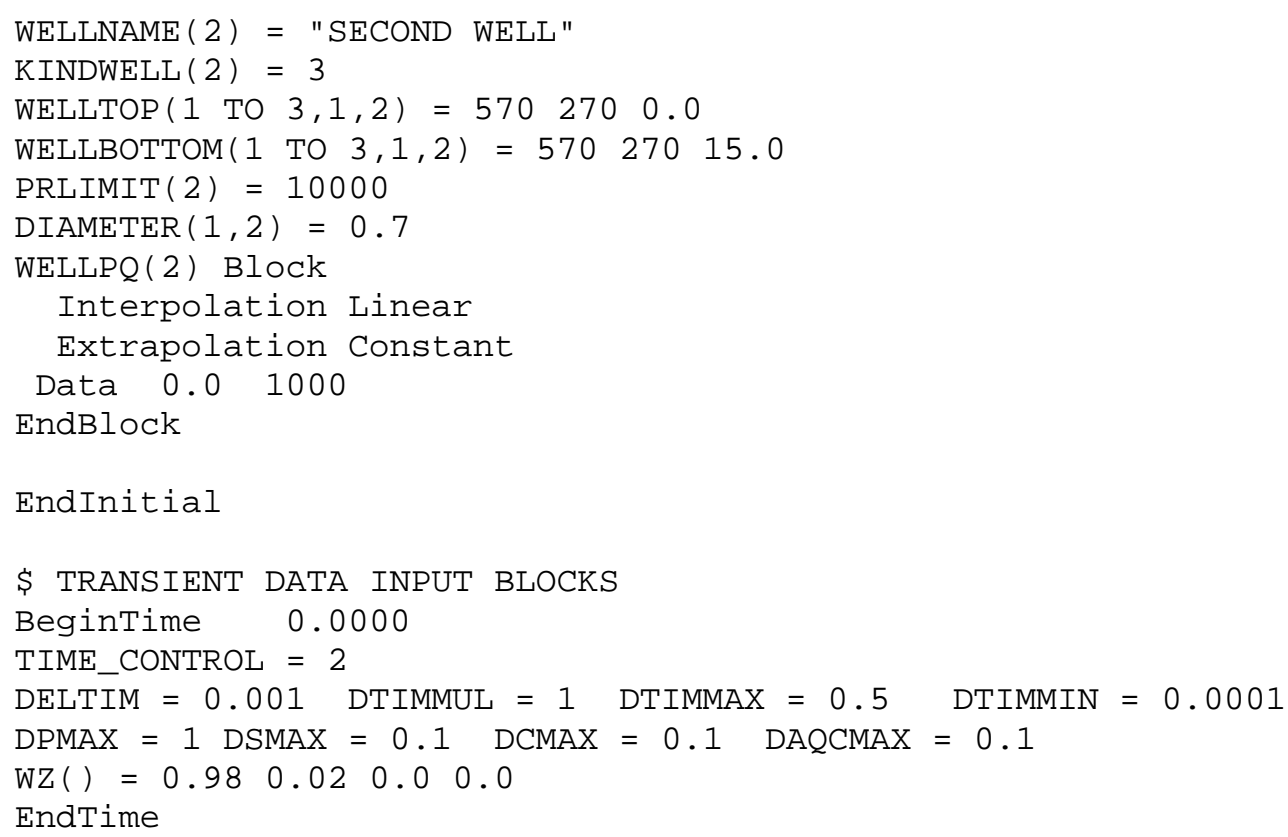

\section{A.2 Coupled Dual Continuum AND EDFM Simulation (Section 8.4.2)}

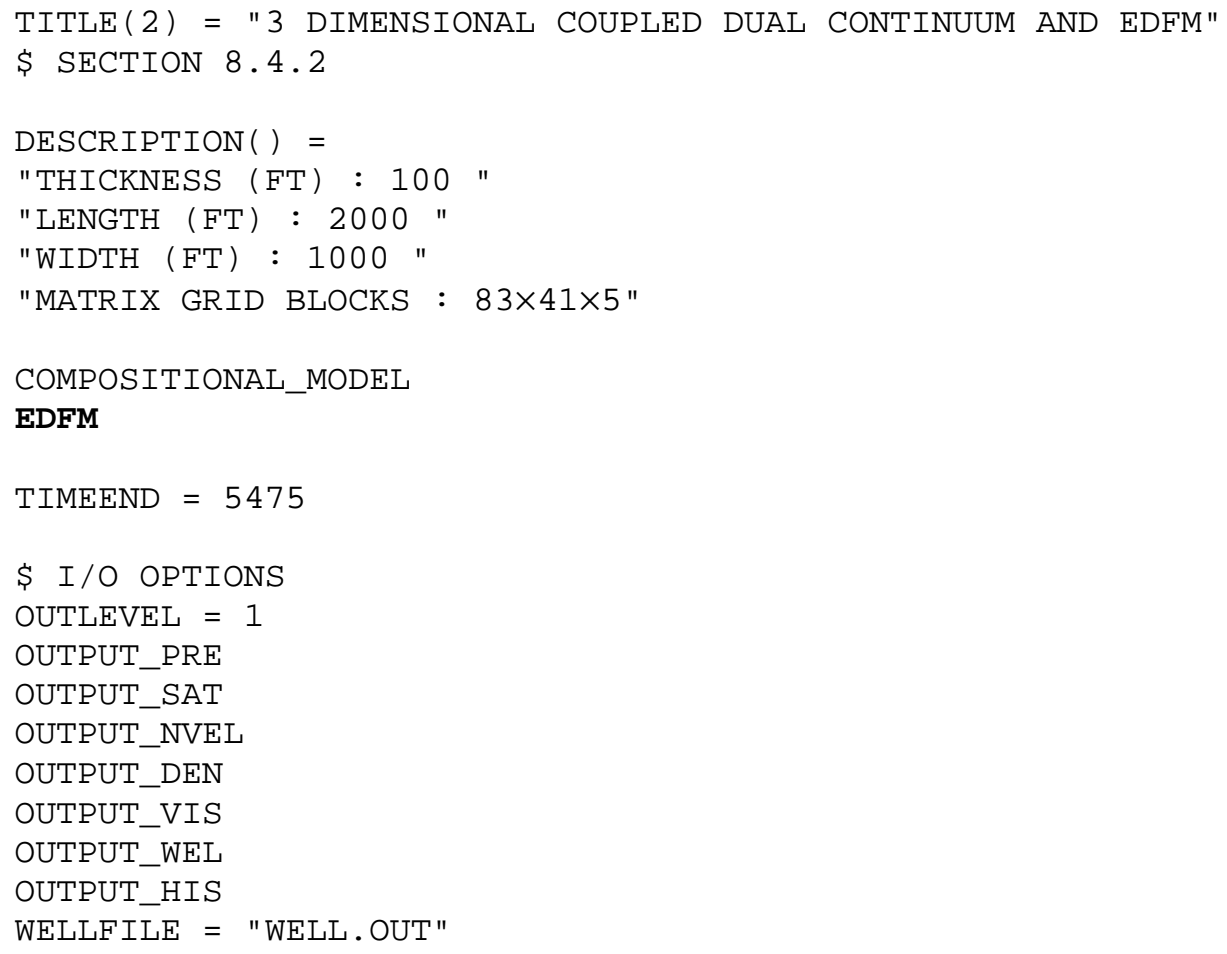




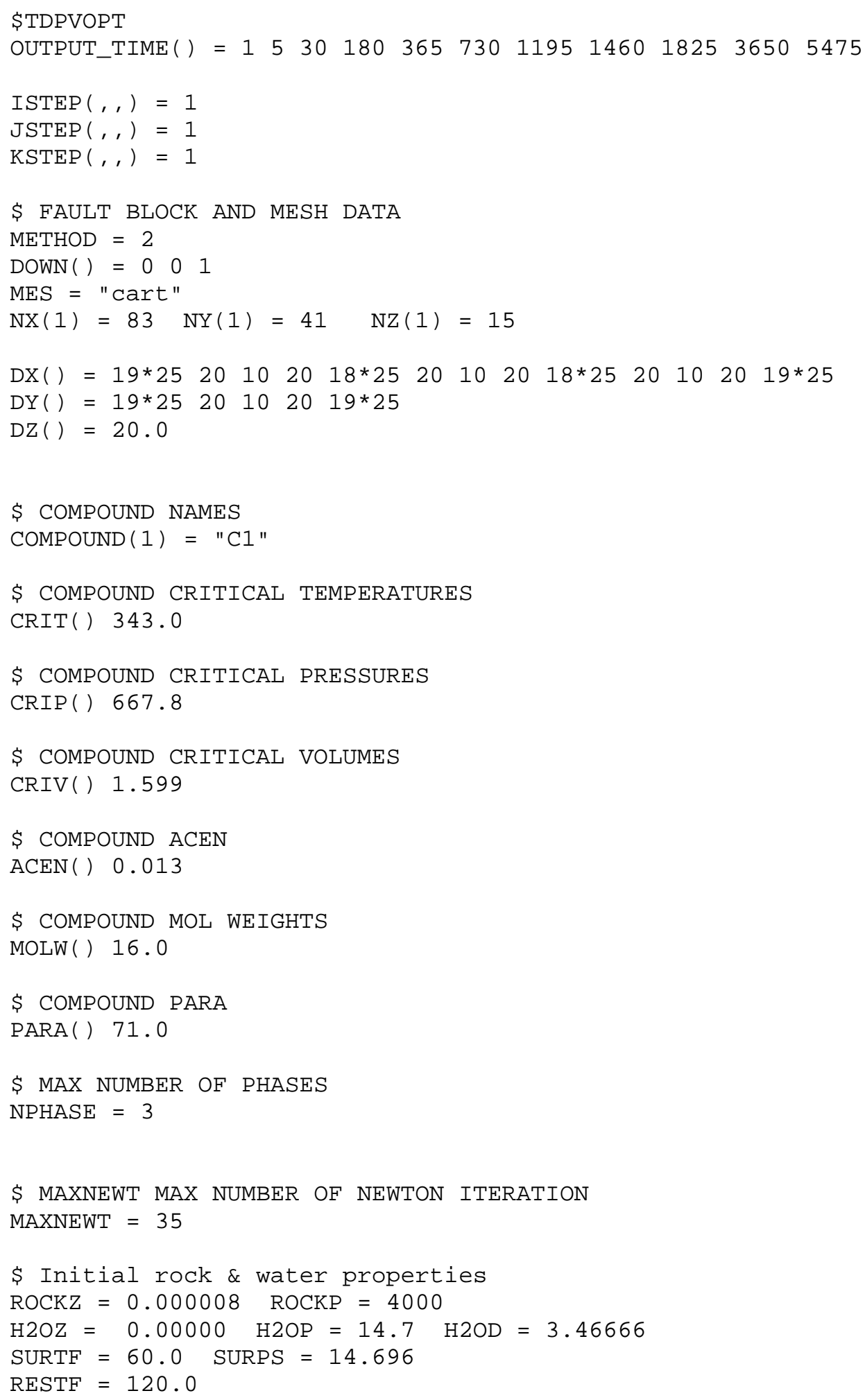




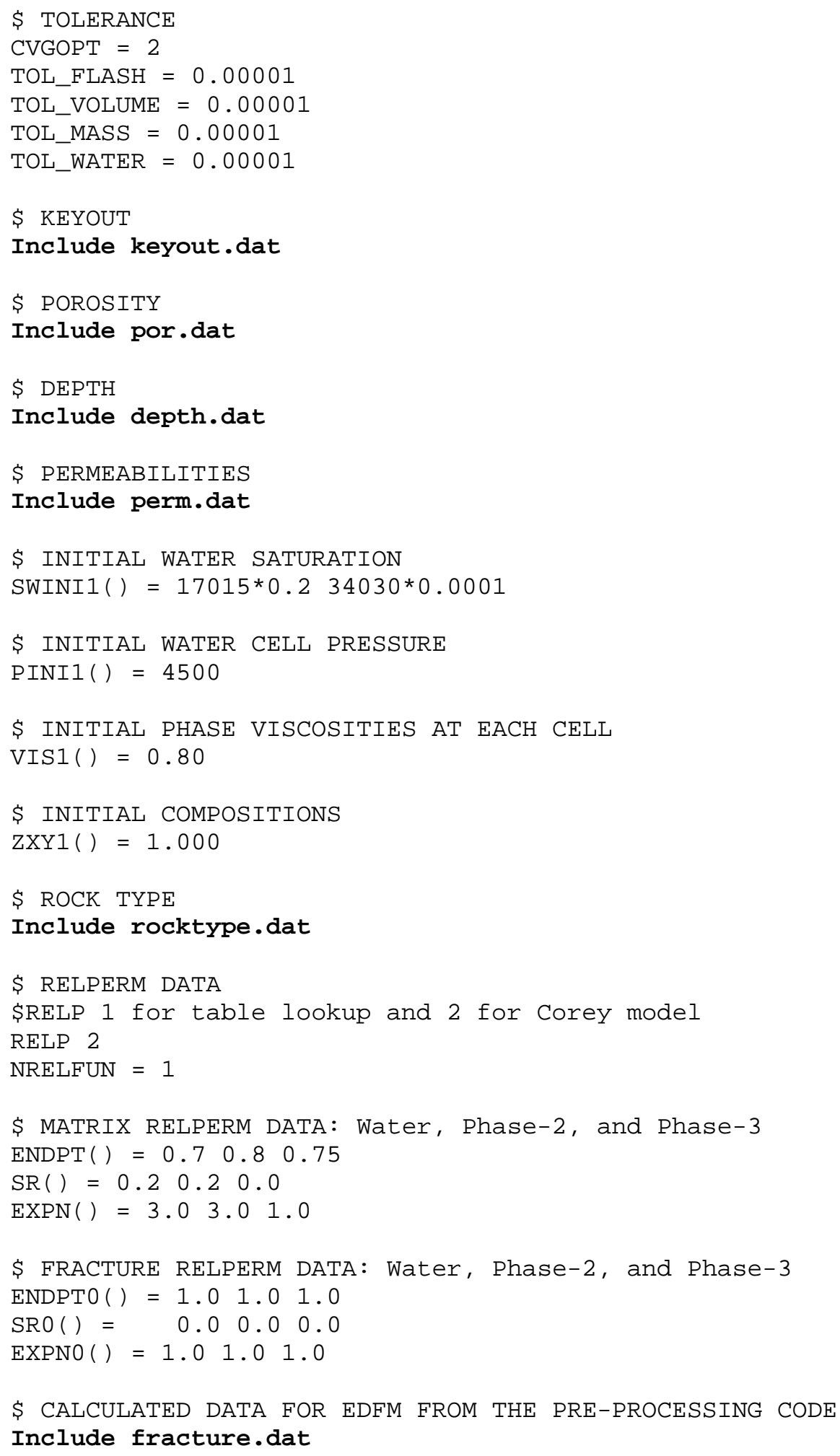




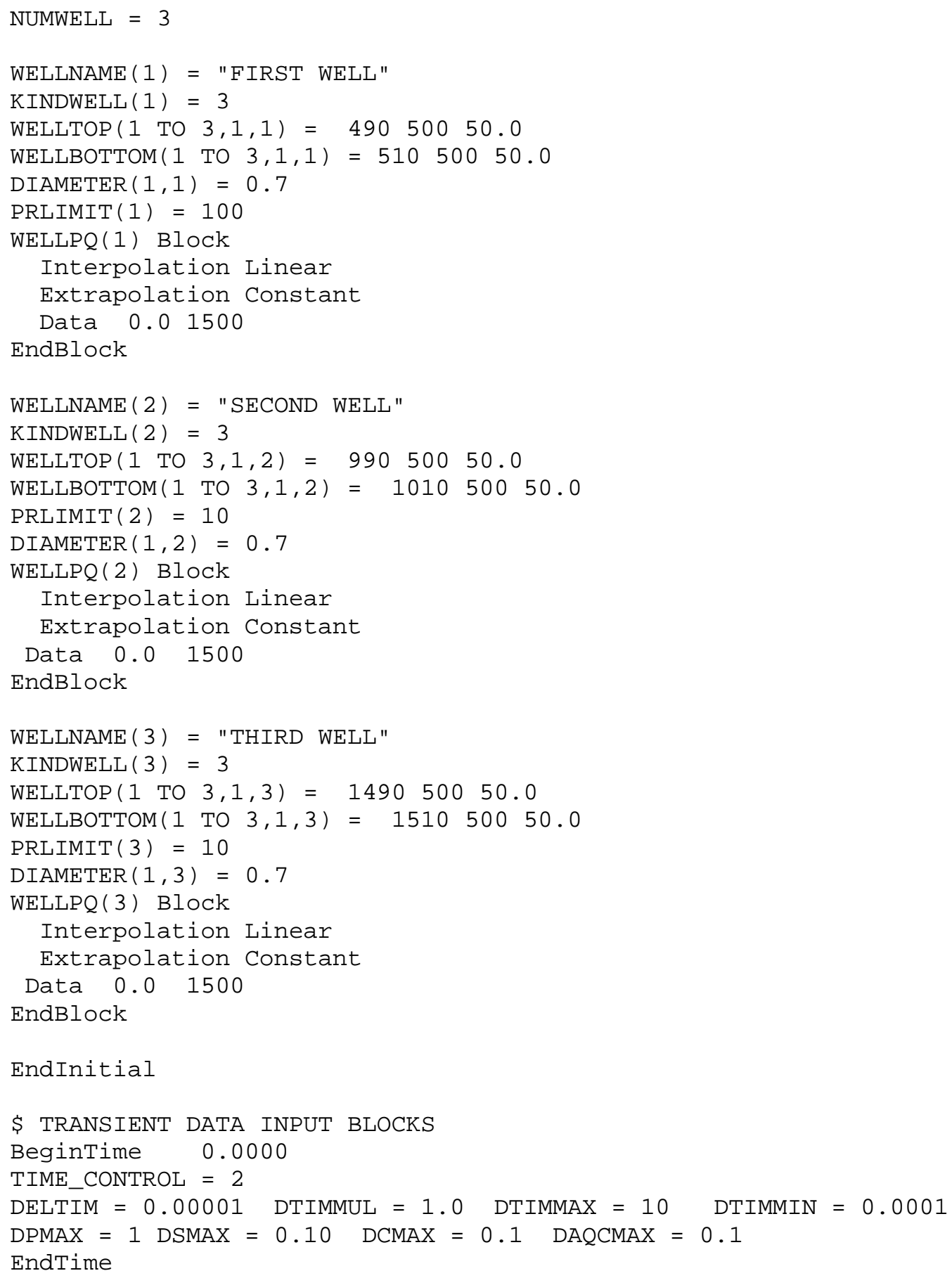




\section{Nomenclature}

\begin{tabular}{|c|c|}
\hline$A$ & area \\
\hline$B$ & formation volume factor \\
\hline C & total compressibility \\
\hline$C_{F D}$ & dimensionless fracture conductivity \\
\hline$d$ & distance \\
\hline$<d>$ & average normal distance \\
\hline$D$ & depth \\
\hline$D_{n}$ & fracture normal closure \\
\hline$D_{\text {nmax }}$ & maximum normal closure \\
\hline$f_{i}^{j}$ & fugacity of component $i$ in phase $j$ \\
\hline$h$ & height \\
\hline$J$ & Jacobian matrix \\
\hline$K_{i}$ & equilibrium ratio of component $i$ \\
\hline$\overline{\bar{k}}$ & absolute permeability tensor \\
\hline$k$ & absolute permeability \\
\hline$k_{r j}$ & relative permeability of phase $j$ \\
\hline$K_{n}$ & normal stiffness \\
\hline$L$ & length \\
\hline$L_{\text {int }}$ & length of intersection line \\
\hline$L_{j}$ & mole fraction of phase $j$ \\
\hline$n$ & unit vector normal to fracture \\
\hline$N_{i}$ & moles of component $i$ per unit pore volume \\
\hline$N_{j}$ & moles of phase $j$ per unit pore volume \\
\hline
\end{tabular}




\begin{tabular}{|c|c|}
\hline$N_{c}$ & number of hydrocarbon components \\
\hline$N_{p}$ & number of hydrocarbon phases \\
\hline$P$ & pressure \\
\hline$P_{p}$ & pore pressure \\
\hline$P_{\text {cow }}$ & oil-water capillary pressure \\
\hline$P_{c o g}$ & oil-gas capillary pressure \\
\hline$q$ & production rate \\
\hline$q_{i}$ & molar rate of component $i$ injected or produced \\
\hline$q_{m f}$ & matrix-fracture flow rate per unit bulk volume \\
\hline$r$ & radius \\
\hline$R$ & residual vector / gas constant \\
\hline$S$ & stress \\
\hline$S_{\text {hmin }}$ & minimum horizontal stress \\
\hline$S_{\text {Hmax }}$ & maximum horizontal stress \\
\hline$S_{n}$ & normal stress \\
\hline$t$ & time \\
\hline$T$ & transmissibility / temperature \\
\hline$S_{w}$ & water saturation \\
\hline$v$ & volume \\
\hline $\bar{v}_{j}$ & molar volume of phase $j$ \\
\hline$V_{b}$ & bulk volume \\
\hline$w$ & volume fraction \\
\hline$x_{i j}$ & mole fraction of component $i$ in phase $j$ \\
\hline$x_{f}$ & fracture half-length \\
\hline$Z$ & compressibility factor \\
\hline
\end{tabular}




\section{Greek Symbols}

$\begin{array}{ll}\sigma & \text { shape factor } \\ \sigma_{n} & \text { effective normal stress } \\ \mu & \text { viscosity } \\ \phi & \text { porosity } \\ \psi & \text { potential } \\ \varphi_{i}^{j} & \text { fugacity coefficient of component } i \text { in phase } j \\ \xi_{j} & \text { molar density of phase } j \\ \gamma_{j} & \text { specific gravity of phase } j \\ \lambda & \text { interporosity flow coefficient } \\ \omega & \text { ratio of fluid capacitance / acentric factor } \\ \omega_{f} & \text { fracture aperture } \\ \delta & \text { binary interaction coefficient }\end{array}$

\section{Subscripts / Superscripts}

$\begin{array}{ll}c & \text { critical } \\ D & \text { dimensionless } \\ i & \text { initial } \\ m & \text { matrix } \\ n n c & \text { non-neighboring connection } \\ f & \text { fracture (continuum-fracture in Chapter 8) } \\ F & \text { fracture (discrete-fracture in Chapter 8) } \\ w & \text { water / well } \\ o & \text { oil } \\ g & \text { gas }\end{array}$




\section{Acronyms}

\begin{tabular}{ll} 
BHP & Bottom-hole Pressure \\
CVFE & Control-Volume Finite-Element \\
DFM & Discrete Fracture Model \\
EDFM & Embedded Discrete Fracture Model \\
EOS & Equation of State \\
GPAS & General Purpose Adaptive Simulator \\
IOR & Improved Oil Recovery \\
IPARS & Integrated Parallel Accurate Reservoir Simulation \\
MINC & Multiple Interacting Continua \\
NNC & Non-Neighboring Connection \\
NFR & Naturally Fractured Reservoir \\
PETSc & Portable Extensible Toolkit for Scientific Computation \\
PV & Pore Volume \\
USDFM & Unstructured Discrete Fracture Model \\
UTCHEM & University of Texas Chemical Simulator \\
UTCOMP & University of Texas Compositional Simulator \\
WI & Wndex \\
\hline
\end{tabular}




\section{References}

Aldejain, A.A., 1999, Implementation of dual-porosity model in a chemical flooding simulator, PhD Dissertation, The University of Texas at Austin.

Al-Kobaisi, M., Kazemi, H., Ramirez, B., Ozkan, E., and Atan, S., 2009, A critical review for proper use of water/oil/gas transfer functions in dual-porosity naturally fractured reservoirs: part II, SPE Reservoir Evaluation and Engineering, Vol. 12, 211-217.

Angerer, E., Horns, S.A., Gaiser, J.E., Walters,R., Bagala, S., and Vetri, L., 2002, Characterization of dipping fractures using PS mode-converted data, SEG Annual International Meeting, Salt Lake City, UT, October 6-12.

Babadagli, T., 2001, Scaling of co-current and counter-current capillary imbibition for surfactant and polymer injection in naturally fractured reservoirs, SPE Journal, Vol. 6, 465-478.

Baca, R.G., Arnett, R.C., and Langford, D.W., 1984, Modeling fluid flow in fractured porous rock masses by finite element techniques, International Journal of Numerical Methods in Fluids, Vol. 4, 337-348.

Bagheri, M. and Settari, A., 2008, Modeling of geomechanics in naturally fractured reservoirs, SPE Reservoir Evaluation and Engineering, Vol. 11, 108-118.

Bai, M., Meng, F., Elsworth, D., Aboulsleiman, Y., and Roegiers, J.C., 1999, Numerical modeling of coupled flow and deformation in fractured rock specimens, International Journal for Numerical and Analytical Methods in Geomechanics, Vol. 23, 141-160.

Balasubramanian, S., 2007, A compositional model for fractured reservoir simulation, $\mathrm{PhD}$ Dissertation, University of Utah.

Balay, S., Gropp, W.D., Curfman McInnes, L., and Smith, B., 1998, PETSc 2.0 user's manual, Argonne National Laboratory, ANL-95/11 - Revision 2.0.22.

Balogun, A., Kazemi, H., Ozkan, E., Al-Kobaisi, M., and Ramirez, B., 2007, Verification and proper use of water-oil transfer function for dual-porosity and dualpermeability reservoirs, SPE 104580, SPE Middle East Oil and Gas Show and Conference, Bahrain, March 11-14.

Bandis, S.C., Lumsden, A.C., and Barton, N.R., 1983, Fundamentals of rock joint deformation, International Journal of Rock Mechanics and Mining Sciences and Geomechanics, Abstract. 20 (6), 249-268. 
Barenblatt, G.E., Zheltov, I.p., and Kochina, I.N., 1960, Basic concepts in the theory of seepage of homogeneous liquids in fissured rocks, Journal of Applied Mathematics and Mechanics, Vol.24, 1286-1303.

Barkve, T. and Firoozabadi, A., 1992, Analysis of reinfiltration in fractured porous media, SPE 24900, SPE Annual Technical Conference and Exhibition, Washington, DC, October 4-7.

Barton, N.R., Bandis, S.C., and Bakhtar, K., 1985, Strength, deformation and conductivity coupling of rock joints, International Journal of Rock Mechanics and Mining Sciences and Geomechanics, Abstract. 22 (3), 121-140.

Beckner, B.L., Firoozabadi, A., and Aziz, K., 1988, Modeling transverse imbibition in double-porosity simulators, SPE 17414, SPE California Regional Meeting, Long Beach, CA, March 23-25.

Beckner, B.L., Chan, H., McDonald, A., and Jones, T., 1991, Simulating naturally fractured reservoirs using a subdomain method, SPE 21241, SPE Reservoir Simulation Symposium, Anaheim, CA, February 17-20.

Belani, A.K. and Jalali-Yazdi, Y., 1988, Estimation of matrix block size distribution in naturally fractured reservoirs, SPE 18171, SPE Annual Technical Conference and Exhibition, Houston, TX, October 2-5.

Beliveau, D., Payne, D.A., and Mundry, M., 1993, Waterflood and $\mathrm{CO}_{2}$ flood of the fractured Midale Field, Journal of Petroleum Technology, Vol. 45, 881-887.

Blaskovich, F.T., Gain, G.M., and Sonier, F., 1983, A multi-component isothermal system for efficient reservoir simulation, SPE 11480, Middle East Oil Technical Conference, Bahrain, March 14-17.

Brooks, R.H. and Corey, A.T., 1964, Hydraulic properties of porous media, Hydrology Paper, No. 3, Colorado State University, Fort Collins, CO.

Chang, Y., 1990, Development and application of an equation of state compositional simulator, PhD Dissertation, The University of Texas at Austin.

Chen, W.H., Wasserman, M.L., and Fitzmorris, R.E., 1987, A thermal simulator for naturally fractured reservoirs, SPE 16008, SPE Reservoir Simulation Symposium, San Antonio, TX, February 1-4.

Chen, J., 1993, New approach to dual-porosity modeling of waterflooding in naturally fractured reservoirs, PhD Dissertation, The University of Texas at Austin.

Chen, C.C. and Raghavan, R., 1997, A multiply-fractured horizontal well in a rectangular drainage region, SPE Journal, Vol. 2, 455-465.

Chin, L., Raghavan, R., and Thomas, L.K., 2000, Fully coupled geomechanics and fluid flow analysis of wells with stress-dependent permeability, SPE Journal, Vol. 5, 32-45. 
Cinco-Ley, H., Samaniego, F.V., and Dominguez, N., 1978, Transient pressure behavior of a well with a finite-conductivity vertical fracture, SPE Journal, Vol. 18, 253264.

Cinco-Ley, H. and Samaniego, F.V., 1981, Transient pressure analysis for fractured wells, SPE Journal, Vol. 33, 1749-1766.

Cinco-Ley, H., Samaniego, F.V., and Kucuk, F., 1985, The pressure transient behavior for naturally fractured reservoirs with multiple block size, SPE 14168, SPE Annual Technical Conference and Exhibition, Las Vegas, NV, September 22-26.

Cipolla, C.L., Lolon, E.P., Erdle, J., and Rubin, B., 2010, Reservoir modeling in shalegas reservoirs, SPE Journal, Vol. 13, 638-653.

Cipolla, C., Weng, X., Mack, M., Ganguly, U., Gu, H., Kreese, O., and Cohen, C., 2011, Integrating micro-seismic mapping and complex fracture modeling to characterize fracture complexity, SPE 140185, SPE Hydraulic Fracturing Technology Conference, The Woodlands, TX, January 24-26.

Coats, K.H., 1989, Implicit compositional simulation of single-porosity and dual-porosity reservoirs, SPE 18427, SPE Reservoir Simulation Symposium, Houston, TX, February 6-8.

Clemo, T. and Smith, L., 1997, A hierarchical model of solute transport in fractured media, Water Resources Research, Vol. 33, 1763-1784.

Darvish, G.R., Lindeberg, E., Holt, T., and Utne, S.A., 2006, Laboratory experiments of $\mathrm{CO}_{2}$ injection into a fractured core, SPE 99649, SPE Symposium on Improved Oil Recovery, Tulsa, OK, April 22-26.

Dean, R.H. and Lo, L., 1988, Simulations of naturally fractured reservoirs, SPE Reservoir Engineering, Vol. 3, 638-648.

DeBaun, D., Byer, T., Childs, P., Chen, J., Saaf, F., Wells, M., Liu, J., Cao, H., Pianelo, L., Tialkraj, V., Crumpton, P., Walsh, D., Yardomian, H., Zorzynski, R., Lim, K.T., Schrader, M., Zapata, V., Noleon, J., and Tchelepi, H., 2005, An extensible architecture for next generation scalable parallel reservoir simulation, SPE 93274, SPE Reservoir Simulation Symposium, The Woodlands, TX, January 31 February 2.

Delshad, M., Pope, G.A., and Sepehrnoori, K., 1996, A compositional simulator for modeling surfactant enhanced aquifer remediation -1 . Formulation, Journal of Contaminant Hydrology, Vol. 23, 303-327.

Dershowitz, B., LaPointe, P., Eiben, T., and Wei, L., 2000, Integration of discrete feature network methods with conventional simulator approaches, SPE Reservoir Evaluation and Engineering, Vol. 3, 165-170.

De Swaan, O.A., 1976, Analytic solutions for determining naturally fractured reservoir properties by well testing, SPE Journal, Vol. 16, 117-122. 
Dindoruk, B. and Firoozabadi, A. 1994, Computation of gas-liquid drainage in fractured porous media recognizing fracture liquid flow, Annual Technical Meeting, Calgary, AB, June 12-15.

Dindoruk, B. and Firoozabadi, A. 1997, Crossflow in fractured/layered media incorporating gravity, viscous, and phase behavior effects, SPE Journal, Vol. 2, 120-135.

Dogru, A.H., Sunaidi, H.A., Fung, L.S., Habiballah, W.A., Al-Zamel, N., and Li, K.G., 2002, A parallel reservoir simulator for large-scale reservoir simulation, $S P E$ Reservoir Evaluation and Engineering, Vol. 7, 11-23.

Evans, K.F., Kohl, T., Hopkirk, R.J., and Rybach, L., 1992, Modeling of energy production from hot dry rock systems, ETH, Zurich, Switzerland.

Fast, R.E., Murter, A.S., and Timmer, R.S., 1994, Description and analysis of cored hydraulic fractures- Lost Hills Field, Kern County, California, SPE Production and Facilities, Vol. 9, 107-114.

Fathi-Najafabadi, N., Han, C., Delshad, M., and Sepehrnoori, K., 2009, Development of a three phase, fully implicit, parallel chemical flood simulator, SPE 119002, SPE Reservoir Simulation Symposium, The Woodlands, TX, February 2-4.

Firoozabadi, A. and Markeset, T., 1994, An experimental study of the gas-liquid transmissibility in fractured porous media, SPE Reservoir Engineering, Vol. 9, 201-207.

Firoozabadi, A. and Markeset, T., 1994, Miscible displacement in fractured porous media- Part I: Experiments, SPE 27743, SPE Symposium on Improved Oil Recovery, Tulsa, OK, April 17-20.

Firoozabadi, A., Markeset, T., and Dindoruk, B., 1997, Viscous displacement in fractured porous media, Journal of Canadian Petroleum Technology, Vol. 36, 50-62.

Fisher, M.K., Wright, C.A., Davidson, B.M., Goodwin, A.K., Fielder, E.O., Buckler, W.S., and Steinberger, N.P., 2005, Integrating fracture-mapping technologies to improve stimulation in the Barnett Shale, SPE Production and Facilities, Vol. 20, 85-93.

Fredd, C.N., McConnell, S.B., Boney, C.L., and England, K.W., 2001, Experimental study of fracture conductivity for water fracturing and conventional fracturing applications, SPE Journal, Vol. 6, 288-298.

Fu, Y., Yang, Y.K., and Deo, 2005, Three-dimensional, three-phase discrete-fracture reservoir simulator based on control volume finite element (CVFE) formulation, SPE 93292, SPE Reservoir Simulation Symposium, Houston, TX, January 31February 2.

Fung, L.K., 1991, Simulation of block-to-block processes in naturally fractured reservoirs, SPE Reservoir Engineering, Vol. 6, 477-484. 
Fung, L.K. and Dogru, A.H., 2008, Distributed unstructured grid infrastructure for complex reservoir simulation, SPE 113906, SPE Europec/EAGE Annual Conference and Exhibition, Rome, Italy, June 9-12.

Gai, X., Dean, R.H., and Wheeler, M.F., 2003, Coupled geomechanical and reservoir modeling on parallel computers, SPE 79700, SPE Reservoir Simulation Symposium, Houston, TX, February 3-5.

Geiger, S., Matthai, S., Niessner, J., and Helmig, R., 2009, Black-oil simulations for three-component, three-phase flow in fractured porous media, SPE Journal, Vol. $14,338-354$.

Gillespie, P.A., Howard, C.B., Walsh, J.J. and Watterson, J., 1993, Measurement and characterization of spatial distributions of fractures, Tectonophysics, Vol. 226, $113-141$.

Gilman, J.R. and Kazemi, H., 1983, Improvements in simulation of naturally fractured reservoirs, SPE Journal, Vol. 23, 695-707.

Gilman, J.R., 1986, An efficient finite-difference method for simulating phase segregation in the matrix blocks in double-porosity reservoirs, SPE Reservoir Engineering, Vol. 1, 403-413.

Gilman, J.R. and Kazemi, H., 1988, Improved calculations for viscous and gravity displacement in matrix blocks in dual-porosity simulators, Journal of Petroleum Technology, Vol. 40, 60-70.

Goodman, R.E., 1974, The mechanical properties of joints, Proceedings of the $3^{\text {rd }}$ ISRM, Denver, Vol. 1A, 127-140.

Grechka, V. and Tsuankin, I., 2004, Characterization of dipping fractures in transversely isotropic background, Geophysical Prospecting, Vol. 68, 1399-1407.

Gringarten, A.C., Ramey, H.J., and Raghavan, R., 1974, Unsteady state pressure distribution created by a well with a single infinite conductivity vertical fracture, SPE Journal, Vol. 14, 347-360.

Gropp, W., Morgan, T., Smith, B., Arbogast, T., Dawson, C.N., Lake, L.W., McKinney, D.C., Pope, G.A., Sepehrnoori, K., and Wheeler, M.F., 1996, New generation framework for petroleum reservoir simulation, First Annual Report, Advanced Technology Initiative, Argonne National Laboratory and The University of Texas at Austin.

Guo, G., Evans, R.D., and Chang, M.M., 1994, Pressure transient behavior for a horizontal well intersecting multiple random discrete fractures, SPE 28390, SPE Annual Technical Conference, New Orleans, LA, September 25-28.

Gutierrez, M., Lewis, R., and Masters, I., 2001, Petroleum reservoir simulation coupling fluid flow and geomechanics, SPE Reservoir Evaluation and Engineering, Vol. 4, $164-172$. 
Haghshenas, M., 2011, Modeling and remediation of reservoir souring, $\mathrm{PhD}$ Dissertation, The University of Texas at Austin.

Hajibeygi, H., Karvounis, D., and Jenny, P., 2011, A hierarchical fracture model for the iterative multiscale finite volume method, Journal of Computational Physics, Vol.230, 8729-8743.

Han, C., Delshad, M., Sepehrnoori, K., and Pope, G.A., 2007, A fully implicit parallel compositional chemical flooding simulator, SPE Journal, Vol. 12, 322-338.

Hearn, C.L., Al-Emadi, A., Worley, P., and Taylor, R.D., 1997, Improved oil recovery in a tight reservoir with conductive faults, ISND Shuaiba, Qatar, SPE 38908, SPE Annual Technical Conference and Exhibition, San Antonio, TX, October 5-8.

Herge, T.M. and Larsen, L., 1994, Productivity of multi-fractured horizontal wells, SPE 28845, SPE European Petroleum Conference, London, UK, October 25-27.

Hill, A.C. and Thomas G.W., 1985, A new approach for simulating complex fractured reservoirs, SPE 13537, SPE Reservoir Simulation Symposium, Anaheim, CA, February 10-13.

Hirasaki, G. and Zhang, D.L., 2004, Surface chemistry of oil recovery from fractured, oil-wet, carbonate formations, SPE Journal, Vol. 9, 151-162.

Horie, T., Firoozabadi, A., and Ishimito, K., 1990, Laboratory studies of capillary interaction in fracture/matrix systems, SPE Reservoir Engineering, Vol. 5, 353360 .

Hoteit, H. and Firoozabadi, A., 2006, Compositional modeling of discrete-fractured media without transfer functions by the discontinuous Galerkin and mixed methods, SPE Journal, Vol. 11, 341-352.

Hui, M., Mallison, B., Lim, K.-T., and Fitzmorris, R., 2008, An innovative workflow to model fractures in a giant carbonate reservoir, IPTC 12572, International Petroleum Technology Conference, Kuala Lumpur, Malaysia, December 3-5.

Hui, M. and Mallison, B., 2009, System and method for predicting fluid flow characteristics within fractured subsurface reservoirs: US Patent Application 2009630709.

Javadpour, F., Fisher, D., and Unsworth, M., 2007, Nanoscale gas flow in shale sediments, Journal of Canadian Petroleum Technology, Vol. 46, 55-61.

Johns, R.T. and Jalali-Yazdi, Y., 1991, Comparison of pressure-transient response in intensely and sparsely fractured reservoirs, SPE Formation Evaluation, Vol. 6, 513-518.

John, A., Han, C., Delshad, M., Pope, G.A., and Sepehrnoori, K., 2005, A new generation chemical-flooding simulator, SPE Journal, Vol. 10, 206-216. 
Juanes, R., Samper, J., and Molinero, J., 2002, A general and efficient formulation of fractures and boundary conditions in the finite element method, International Journal of Numerical Methods in Engineering, Vol. 54, 1751-1774.

Karimi-Fard, M. and Firoozabadi, A., 2003, Numerical simulation of water injection in fractured media using the discrete-fractured model and the Galerkin method, SPE Journal, Vol. 6, 117-126.

Karimi-fard, M., Durlofsky, L.J., and Aziz, K., 2004, An efficient discrete-fracture model applicable for general purpose reservoir simulators, SPE Journal, Vol. 9, 227236.

Kazemi, H., 1969, Pressure transient analysis of naturally fractured reservoirs with uniform fracture distribution, SPE Journal, Vol. 9, 451-462.

Kazemi, H., Merrill, L.S., Porterfield, K.L., and Zeman, P.R., 1976, Numerical simulation of water-oil flow in naturally fractured reservoirs, SPE Journal, Vol. $16,317-326$.

Kazemi, H. and Merrill, L.S., 1979, Numerical simulation of water imbibition in fractured cores, SPE Journal, Vol. 19, 175-182.

Khalili, N., 2008, Two-phase fluid flow through fractured porous media with deformable matrix, Water Resources Research, Vol. 44 (5).

Kleppe, J. and Morse, R.A., 1974, Oil production from fractured reservoirs by water displacement, Fall Meeting of SPE-AIME, Houston, TX, October 6-9.

Kim, J.G. and Deo, M.D., 2000, Finite element discrete-fracture model for multiphase flow in porous media, AIChE Journal, Vol. 46, 1120-1130.

Kyte, J.R., 1970, A centrifuge method to predict matrix-block recovery in fractured reservoirs, SPE Journal, Vol. 10, 164-170.

Lee, B.Y. and Tan, T.B., 1987, Application of a multiple porosity/permeability simulator in fractured reservoir simulator, SPE 16009, SPE Reservoir Simulation Symposium, San Antonio, February 1-4.

Lee, S.H. and Milliken, W.J., 1993, The productivity index of an inclined well in finitedifference reservoir simulation, SPE 25247, SPE Reservoir Simulation Symposium, New Orleans, LA, February 28 - March 3.

Lee, S.H., Jensen, C.L., and Lough, M.F., 2000, Efficient finite-difference model for flow in a reservoir with multiple length-scale fractures, SPE Journal, Vol. 5, 268-275.

Lee, S.H., Lough, M.F., and Jensen, C.L., 2001, Hierarchical modeling of flow in naturally fractured formations with multiple length scales, Water Resources Research, Vol. 37, 443-455. 
Li, H., Putra, E., Schechter, D.S., and Grigg, R.B., 2000, Experimental investigation of $\mathrm{CO}_{2}$ gravity drainage in a fractured system, SPE 64510, SPE Asia Pacific Oil and Gas Conference and Exhibition, Brisbane, Australia, October 16-18.

Li, L. and Lee, S.H., 2008, Efficient field-scale simulation of black oil in a naturally fractured reservoir through discrete fracture networks and homogenized media, SPE Reservoir Evaluation and Engineering, Vol. 11, 750-758.

Lim, K.T. and Aziz, K., 1995, Matrix-fracture transfer shape factors for dual porosity simulators, Journal of Petroleum Science and Engineering, Vol. 13, 169-178.

Lim, K.T., Hui, M., and Mallison, B., 2009, A next-generation reservoir simulator as an enabling technology for a complex discrete fracture modeling workflow, SPE 124980, SPE Annual Technical Conference and Exhibition, New Orleans, LA, October 4-7.

Litvak, B.L., Satter, A., and Etebar, S., 1985, An analysis of naturally fractured reservoir performance using a novel fractured reservoir simulator, Reservoir Characterization Technical Conference, Dallas, TX, April 29 - May 1.

Lorenz, J.C., 1999, Stress sensitive reservoirs, Journal of Petroleum Technology, Vol.51, 61-63.

Lough, M.F., Lee, S.H., and Kamath, J., 1997, A new method to calculate effective permeability of gridblocks used in the simulation of naturally fractured reservoirs, SPE Reservoir Engineering, Vol. 12, 219-224.

Lough, M.F., Lee, S.H., and Kamath, J., 1998, An efficient boundary integral formulation for flow through fractured porous media, Journal of Computational Physics, Vol. $143,462-483$.

Makurat, A. and Gutierrez, M., 1996, Fracture flow and fracture cross flow experiments, SPE 36732, SPE Annual Technical Conference and Exhibitions, Denver, CO, October 6-9.

Mallison, B., Hui, M.H., and Narr, W., 2010, Practical gridding algorithms for discretefracture modeling workflows, European Conference On the Mathematics of Oil Recovery, Oxford, UK, September 6-9.

Maloney, D.R., Gall, B.L., and Raible, C.J., 1989, Non-darcy gas flow through propped fractures: effects of partial saturation, gel damage, and stress, SPE Production Engineering, Vol. 4, 417-422.

Marcondes, F., Varavei, A., and Sepehrnoori, K., 2010, An element-based finite-volume method approach for naturally fractured compositional reservoir simulation, Brazilian Thermal Sciences Meeting, Uberlandia, Brazil, December.

Matthai, S., Menzentsev, A., and Belayneh, M., 2007, Finite-element node-centered finite-volume two-phase-flow experiments with fractured rock represented by 
unstructured hybrid-element meshes, SPE Reservoir Evaluation and Engineering, Vol. 10, 740-756.

Mattax, C.C. and Kyte, J.R., 1962, Imbibition oil recovery from fractured, water-drive reservoir, SPE Journal, Vol. 2, 177-184.

Mavor, M.J. and Cinco-Ley, H., 1979, Transient pressure behavior of naturally fractured reservoirs, SPE 7977, SPE California Regional Meeting, Venture, CA, April 1820.

Maxwell, S.C., Urbancic, T.I., steinsberger, N.P., and Zinno, R., 2002, Microseismic imaging of hydraulic fracture complexity in the Barnett shale, SPE 77440, SPE Annual Technical Conference and Exhibition, San Antonio, TX, September 29October 2.

Mehra, R.K., Heidman, R.A., and Aziz, K., 1983, An accelerated successive substitution algorithm, Canadian Journal of Chemical Engineering, Vol. 61, 590-596.

Michelsen, M.K., 1982, The isothermal flash problem: Part I - Stability, Fluid Phase Equilibria, Vol. 6, 1-19.

Moench, A.F., 1984, Double-porosity models for a fissured groundwater reservoir with fracture skin, Water Resources Research, Vol. 20, 831-846.

Monteagudo, J. and Firoozabadi, A., 2004, Control-Volume method for numerical simulation of two-phase immiscible flow in two- and three- dimensional discretefractured media, Water Resources Research, Vol. 40, 1-20.

Monteagudo, J. and Firoozabadi, A., 2007, Control-volume model for simulation of water injection in fractured media: Incorporating matrix heterogeneity and reservoir wettability effects, SPE Journal, Vol. 12, 355-366.

Monteagudo, J., Rodriguez, A., and Florez, H., 2011, Simulation of flow in discrete deformable fractured porous media, SPE Reservoir Simulation Symposium, The Woodlands, TX, February 21-23.

Naimi-Tajdar, R., Han, C., Sepehrnoori, K., Arbogast, T.J., and Miller A.M., 2007, A fully implicit, compositional, parallel simulator for IOR processes in fractured reservoirs, SPE Journal, Vol. 12, 367-381.

Nalla, G., 2002, Implementation of chemical flooding module into the general purpose adaptive simulator, MS Thesis, The University of Texas at Austin.

Najurieta, H.L., 1980, A theory for pressure transient analysis in naturally fractured reservoirs, SPE Journal, Vol. 32, 1241-1250.

Noorishad, J. and Mehran, M., 1982, An upstream finite-element method for solution of transient transport equation in fractured porous media, Water Resources Research, Vol. 18, 588-596. 
Oda, M., 1985, Permeability tensor for discontinuous rock masses, Geotechnique, Vol. $35,483-495$.

Odeh, A.S., 1965, Unsteady-state behavior of naturally fractured reservoirs, SPE Journal, Vol. 5, 60-66.

Odling, N.E., Gillespie, P., Bourgine, B., Castaing, C., Chiles, J.P., Christensen, N.P.,Fillion, E., Genter, A., Olsen, C., Thrane, L., Trice, R., Aarseth, E., Walsh, J.J., and Watterson, J., 1999, Variations in fracture system geometry and their implications for fluid flow in fractured hydrocarbon reservoirs. Petroleum Geoscience, Vol. 5, 373-384.

Odling, N. E., 1997, Scaling and connectivity of joint systems in sandstones from western Norway, Journal of Structural Geology, Vol. 19, 1257-1271.

Ozkan, E. and Raghavan, R., 1991, New solutions for well-test-analysis problems, Part 2: Computational considerations and applications, SPE Formation Evaluation, Vol. 6, 369-378.

Pao, W.K. and Lewis, R.W., 2002, Three-dimensional finite element simulation of threephase flow in a deforming fissured reservoir, Computer Methods in Applied Mechanics and Engineering, Vol. 191, 2631-2659.

Parashar, M., Wheeler, J.A., Pope, G., Wang, K., and Wang, P., 1997, A new generation EOS compositional reservoir simulator: Part II - Framework and multiprocessing, SPE 37977, SPE Reservoir Simulation Symposium, Dallas, TX, June 8-11.

Peaceman, D.W., 1978, Interpretation of well-block pressures in numerical reservoir simulation, SPE Journal, Vol. 18, 183-194.

Peaceman, D.W., 1983, Interpretation of well-block pressures in numerical reservoir simulation with non-square grid blocks and anisotropic permeability, SPE Journal, Vol. 23, 531-543.

Peng, D.Y. and Robinson, D.B., 1976, A new two-constant equation of state, Industrial and Engineering Chemical Fundamentals, Vol. 15, 59-64.

Perschke, D. R., Chang, Y., Pope, G.A., and Sepehrnoori, K., 1989, Comparison of phase behavior algorithms for an equation-of-state compositional simulator, SPE 19443, Submitted to SPE.

Philip, Z.G., 2003, Incorporating subcritical crack growth mechanics into natural fracture characterization for improved reservoir simulation, $\mathrm{PhD}$ Dissertation, The University of Texas at Austin.

Pinzon, C.L., Chen, H., and Tuefel, L.W., 2000, Complexity of well testing analysis of naturally fractured gas-condensate wells in Colombia, SPE 59013, SPE International Petroleum Conference and Exhibition, Mexico, February 1-3. 
Pooladi-Darvish, M. and Firoozabadi, A., 2000, Experiments and modeling of water injection in water-wet fractured porous media, Journal of Canadian Petroleum Technology, Vol. 39, 31-42.

Por, G.J., Boerrigter, P., Maas, J.G., and de Vries, A., 1989, A fractured reservoir simulator capable of modeling block-block interaction, SPE 19807, SPE Annual Technical Conference and Exhibition, San Antonio, TX, October 8-11.

Pruess, K. and Narasimhan, T.N., 1985, A practical method for modeling fluid and heat flow in fractured porous media, SPE Journal, Vol. 2, 14-26.

Raghavan, R., Chen, C.C., and Agarwal, B., 1997, An analysis of horizontal wells intercepted by multiple fractures, SPE Journal, Vol. 2, 235-245.

Raghavan, R. and Chin, L., 2004, Productivity changes in reservoirs with stressdependent permeability, SPE Reservoir Evaluation and Engineering, Vol. 7, 308315.

Ramirez, B., Kazemi, H., Al-Kobaisi, M., and Ozkan, E., 2009, A critical review for proper use of water/oil/gas transfer functions in dual-porosity naturally fractured reservoirs: part I, SPE Reservoir Evaluation and Engineering, Vol. 12, 200-210.

Ranjbar, E., Hassanzadeh, H., and Chen, Z., 2011, Effect of fracture pressure depletion regimes on the dual-porosity shape factor for flow of compressible fluids in fractured porous media, Advances in Water Resources, Vol. 34, 1681-1693.

Reichenberger, V., Jakobs, H., Bastian, P., and Helmig, R., 2006, A mixed-dimensional finite volume method for two-phase flow in fractured porous media, Advances in Water Resources, Vol. 29, 1020-1036.

Reiss, L., 1980, The reservoir engineering aspects of fractured formations, Gulf Publishing Co., Houston.

Rodriguez, F., Horne, R.N., and Cinco-Ley, H., 1984a, Partially penetrating fractures: pressure transient analysis of an infinite conductivity fracture, SPE 12743, SPE California Regional Meeting, Long Beach, CA, April 11-13.

Rodriguez, F., Horne, R.N., and Cinco-Ley, H., 1984b, Partially penetrating fractures: pressure transient analysis of a finite conductivity fracture, SPE 13057, SPE Annual Technical Conference and Exhibition, Houston, TX, September 16-19.

Rossen, R.H., 1977, Simulation of naturally fractured reservoir with semi-implicit source terms, SPE Journal, Vol. 17, 201-210.

Rossen, R.H. and Shen, E.I., 1989, Simulation of gas-oil drainage and water-oil imbibition in naturally fractured reservoirs, SPE Reservoir Engineering, Vol. 4, 464-470. 
Rubin, B., 2007, Simulating gravity drainage and reinfiltration with a subdomain-dualpermeability hybrid fracture model, SPE 106191, SPE Reservoir Simulation Symposium, Houston, TX, February 26-28.

Rubin, B., 2010, Accurate simulation of non-darcy flow in stimulated fractured shale reservoirs, SPE 132093, SPE Western Regional Meeting, Anaheim, CA, May 2729.

Saidi, A.M., 1983, Simulation of naturally fractured reservoirs, SPE 12270, SPE Reservoir Simulation Symposium, San Francisco, CA, November 15-18.

Samier, P., Onaisi, A., and Fontaine, G., 2006, Comparisons of uncoupled and various coupling techniques for practical field examples, SPE Journal, Vol.11, 89-102.

Schechter, D.S. and Guo, B., 1998, An integrated investigation for design of a $\mathrm{CO}_{2}$ pilot in the naturally fractured Spraberry Trend Area, West Texas, SPE 39881, International Petroleum Conference and Exhibition of Mexico, Villahermosa, Mexico, March 3-5.

Settari, A. and Mourits, F.M., 1998, A coupled reservoir and geomechanical simulation systems, SPE Journal, Vol. 3, 219-226.

Shchipanov, A.A. and Nazarov, A., 2005, Simulation of fluid flow in fractured reservoirs subject to deformability, Proceedings of the AAPG International Conference and Exhibition, Paris, France.

Shchipanov, A.A. and Rusakov, S.V., 2008, Transient pressure well test analysis based on compressible discrete fracture network, European Conference on the Mathematics of Oil Recovery, Bergen, Norway, September 8-11.

Sonier, F., Souillard, P., and Blaskovich, F.T., 1988, Numerical simulation of naturally fractured reservoirs, SPE Reservoir Engineering, Vol. 3, 1114-1122.

Spivey, J.P. and Lee, W.J., 2000, Pressure transient response for a naturally fractured reservoir with a distribution of block sizes, SPE Rocky Mountain Regional Symposium, Denver, CO, March 12-15.

Stehfest, H., 1970, Algorithm 368: Numerical inversion of Laplace transform, Communications of the ACM, Vol. 13, 47-49.

Tan, C.T. and Firoozabadi, A., 1995, Theoretical analysis of miscible displacement in fractured porous media by a one-dimensional model - Part 2: Features, Journal of Canadian Petroleum Technology, Vol. 34, 28-35.

Tao, Q., Economides, C.A., and Ghasemi, A., 2009, Investigation of stress-dependent fracture permeability in naturally fractured reservoirs using a fully coupled poroelastic displacement discontinuity model, SPE 124745, SPE Annual Technical Conference and Exhibitions, New Orleans, LA, October 4-7. 
Tarahhom, F., 2008, Development of an implicit full-tensor dual-porosity compositional reservoir simulator, $\mathrm{PhD}$ Dissertation, The University of Texas at Austin.

Thomas, L.K., Dixon, T., and Pierson, R.G., 1983, Fractured reservoir simulation, SPE Journal, Vol. 23, 42-54.

Tiab, D. and Puthigai, S.K., 1988, Pressure derivative type-curves for a vertically fractured well, SPE Formation Evaluation, Vol. 3, 156-158.

Trangenstein, J.A., 1987, Customized minimization techniques for phase equilibrium computation in reservoir simulation, Chemical Engineering Science, Vol. 42, 2847-2863.

Trivedi, J.J. and Babadagli, T., 2006, Efficiency of miscible displacement in fractured porous media, SPE 100411, SPE Western Regional Meeting, Anchorage, Alaska, May 8-10.

Uleberg, K. and Kleppe, J., 1996, Dual porosity, dual permeability formulation for fractured reservoir simulation, Norwegian University of Science and Technology, Trondheim RUTH Seminar, Stavanger.

Varavei, A., 2009, Development of an equation-of-state thermal flooding simulator, $\mathrm{PhD}$ Dissertation, The University of Texas at Austin.

Walsh, J.J. and Watterson, J., 1988, Dips of normal faults in British coal measures and other sedimentary sequences, Journal of Geologic Society, Vol. 145, 859-873.

Wan, J. and Aziz, K., 2002, Semi-analytic well model of horizontal wells with multiple hydraulic fractures, SPE Journal, Vol. 7, 437-445.

Wang, P., Yotov, I., Wheeler, M. F., Arbogast, T., Dawson, C., Parashar, M., and Sepehrnoori, K., 1997, A new generation EOS compositional reservoir simulator: Part I- Formulation and discretization, SPE 37979, SPE Reservoir Simulation Symposium, Dallas, TX, June 8-11.

Wang, P., Balay, S., Sepehrnoori, K., Wheeler, J., Abate, J., Smithe, B., and Pope, G.A., 1999, A fully implicit parallel EOS compositional simulator for large scale reservoir simulation, SPE 51885, SPE Reservoir Simulation Symposium, Houston, TX, February 14-17.

Warpinski, N.R., Lorenz, J.Z., Branagan, P.T., Myal, F.R., and Gall, B.L., 1993, Examination of a cored hydraulic fracture in a deep gas well, SPE Production and Facilities, Vol. 8, 150-158.

Warren, J.E. and Root, P.J., 1963, The behavior of naturally fractured reservoirs, SPE Journal, Vol. 3, 245-255.

Weng, X., Kreese, C., Cohen, R.W., and Gu, H., 2011, Modeling of hydraulic fracture network propagation in a naturally fractured formation, SPE Production and Operations, Vol. 26, 368-380. 
Wheeler, M.J., Arbogast, T., Bryant, S., Eaton, J., Lu, Q., Peszynska, M., and Yotoy, I., A parallel multiblock/multidomain approach for reservoir simulation, SPE 51884, SPE Reservoir Simulation Symposium, Houston, TX, February 14-17.

Wilbur, C. and Amedei, B., 1990, Flow pump measurement of fracture transmissivity as a function of normal stress, US Symposium on Rock Mechanics, Golden, CO, June 18-20.

Wit, K., Clemens, T., and Rijkels, L., 2002, Simulation of gas/oil gravity drainage in a stack of interacting blocks: Pseudo relations for a limited number of gridblocks, SPE 77722, SPE Annual Technical Conference and Exhibition, San Antonio, TX, September 29 - October 2.

Wu, Y.S. and Pruess, K., 1988, A multiple-porosity method for simulation of naturally fractured petroleum reservoirs, SPE Reservoir Engineering, Vol. 3, 327-336.

Yanze, Y. and Clemens, T., 2012, The role of diffusion for non-equilibrium gas injection into a fractured reservoir, SPE Reservoir Evaluation and Engineering, Vol. 15, 60-71.

Zhang, K., Wu, Y.S., Ding, C., Pruess, K., and Elmroth, E., 2001, Parallel computing techniques for large-scale reservoir simulation multi-component and multiphase fluid flow, SPE 66343, SPE Reservoir Simulation Symposium, Houston, TX, February 11-14.

Zhao, Y. and Chen, M., 2006, Fully coupled dual porosity model for anisotropic formations, International Journal of Rock Mechanics and Mining Sciences, Vol. $43,1128-1133$. 